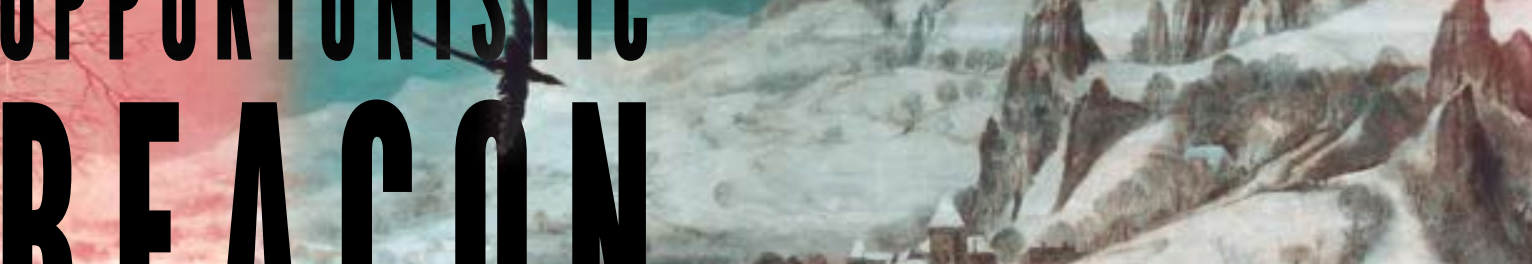

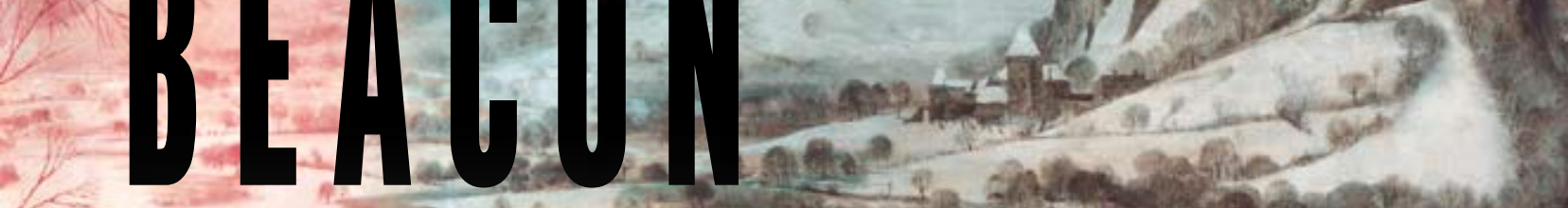

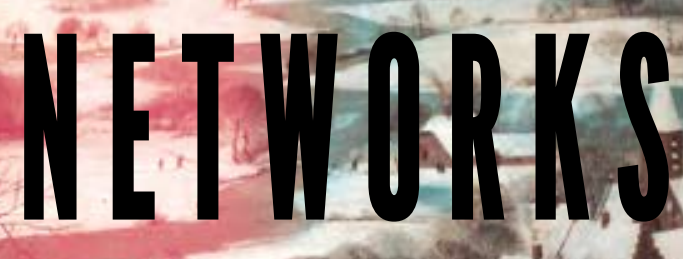

- OKALIÜPKSS,

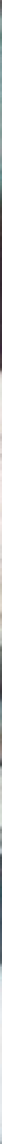




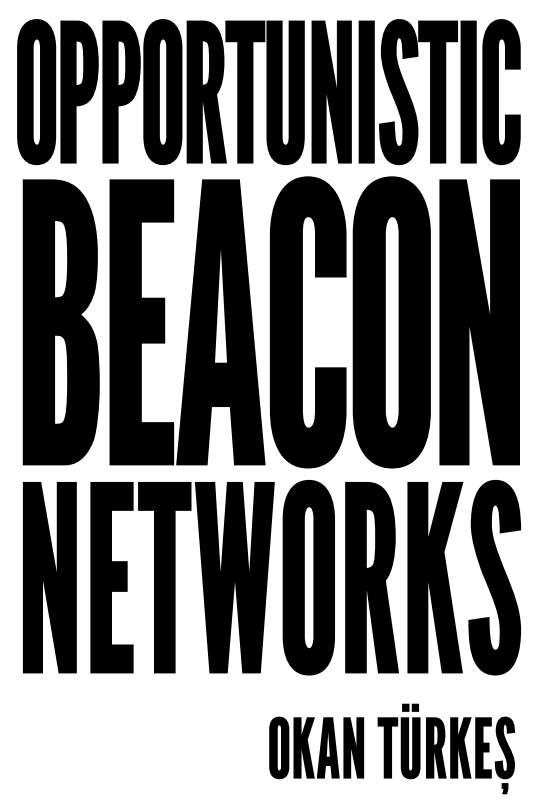


This research is supported by the SenSafety project (Po8-SENSA) within the context of the Dutch National Program COMMIT.

\section{CTIT}

ISSN

$1381-3617$

Cover Painting

The Hunters in the Snow

\section{CTIT PHD THESIS SERIES NO. 16-395}

Centre for Telematics and Information Technology

University of Twente, P.O. Box 217, 7500 AE, Enschede, NL

\section{Print

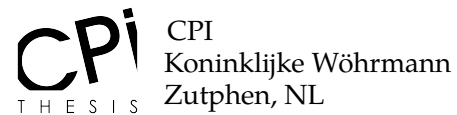

ISBN

978-90-365-4134-3

\section{Cover Artist}

Pieter Bruegel, the Elder

\section{DOI}

10.3990/1.9789036541343

\section{Cover Design}

Gözde \& Okan Türkeş

OKAN TÜRKeş @ 2016 ENSCHEDE, NL

All rights reserved. No part of this book may be reproduced or transmitted, in any form or by any means, electronic or mechanical, including photocopying, microfilming, and recording, or by any information storage or retrieval system, without the prior written permission of the author.

The original work used in the cover design has been identified as being free of known restrictions under copyright law, including all related and neighboring rights. The work is in the collection of the Kunsthistorisches Museum in Vienna, Austria. 


\title{
OPPORTUNISTIC BEACON NETWORKS
}

\author{
DISSERTATION
}

TO OBTAIN

THE DEGREE OF DOCTOR AT THE UNIVERSITY OF TWENTE, ON THE AUTHORITY OF THE RECTOR MAGNIFICUS,

PROF. DR. H. BRINKSMA,

ON ACCOUNT OF THE DECISION OF THE GRADUATION COMMITTEE, TO BE PUBLICLY DEFENDED

ON WEDNESDAY 1 JUNE 2016 AT 14:45

BY

OKAN TÜRKEŞ

BORN ON 8 SEPTEMBER 1986

IN SAMSUN, TURKEY 


\section{THIS DISSERTATION IS APPROVED BY}

Prof. Dr. Paul J. M. Havinga (promotor)

Ir. Hans Scholten (referent)

\section{Graduation Committee}

Prof. Dr. Peter M. G. Apers Prof. Dr. Paul J. M. Havinga Ir. Hans Scholten

Prof. Dr. Maarten R. van Steen

Prof. Dr. Hans van den Berg

Prof. Dr. Ignas G. M. M. Niemegeers

Prof. Dr. Sonia M. Heemstra

Prof. Dr. Şebnem Baydere

University of Twente, chairman \& secretary

University of Twente, promotor

University of Twente, referee

University of Twente

University of Twente

Eindhoven University of Technology

Eindhoven University of Technology

Yeditepe University 

$\tau_{0}$

my parents

To

my sister

\& To

my wife 
The medium is the message.

- Marshall McLuhan 


\section{Acknowledgments}

Ars Longa, Vita Brevis. This thesis is just an epitome of a great deal of enthusiasm and effort, that are not merely scientific, also related to a precious collection of arts I acquired, experienced, and perfected in my most memorable years in the Netherlands. The art of harmony, the art of collaboration, the art of communication, the art of searching, the art of working hard, the art of caring, and most importantly, the art of turning all these arts into a meaning, that is the art of making life valuable together with people around me. I benefited greatly from the support of many wonderful people whom I must credit for helping me build another milestone in my life. It is now time to acknowledge.

Many ideas presented throughout this thesis emerged from the rewarding discussions with my supervisors Paul Havinga and Hans Scholten. First and foremost, with my deepest gratitude, I would like to thank my promotor Paul Havinga for allowing me the opportunity and freedom to do this research. It was a great pleasure and honor to work under his impeccable guidance, constant support, and great patience. His inspiring and distinctive advices widened my academic horizons. I also convey my sincere thanks to my daily supervisor Hans Scholten for his warm friendship and encouraging support during this research. Additional thanks to him for translating the thesis abstract into Dutch. Special thanks go to Nirvana Meratnia who always showed her kindness to solve any difficulty came my way, or to show me the right way.

I am forever indebted to Şebnem Baydere, who conduced to my postgraduate research abroad, for her endless support. Next, I would very much like to thank Sonia Heemstra, Ignas Niemegeers, Maarten van Steen, Hans van den Berg, and again Şebnem Baydere for being part of my graduation committee. I feel honored to have such experts in my defence.

Having contributed directly to my research, all the co-authors of papers published and used as basis for this thesis deserve a special acknowledgement: Nirvana Meratnia, Kyle Zhang, and Fatjon Seraj. 
To all Pervasive Systems group members, I would like to leave my gratitude here for providing such a peaceful working atmosphere.

Words fall short to tell my appreciation to my friend Fatjon Seraj. My acquaintance with him began in front of a Dutch panorama during an intimate talk, since then our cordiality continued and increased with other panoramic talks about almost everything on life and research. His true friendship, his moral support, his savoir-faire helped me a lot during my hardest times, contributed to my research always positively. I feel fortunate to had the pleasure of sharing time with Kyle Zhang, a full-stack engineer, a true friend always eager to help me anytime. Many thanks to him for accepting to be my paranymph at my defense. My thanks are also due to Eyuel D. Ayele for his kindness in accepting to be my paranymph. I am deeply grateful to Mitra Baratchi for her friendship and advises, to Siavash Aflaki, Vignesh R. K. Ramachandran, Wouter van Kleunen, and Muhammad Shoaib for the very nice moments. At the outset of this journey, I had fruitful discussions with Juan Garcia, Ramon S. Schwartz, Majid Bahrepour, Zahra Taghikhaki, Alireza Masoum, Özlem Durmaz Incel, Bram J. Dil, Berend Jan van der Zwaag, and Arta Dilo, all of whom were very encouraging. I also wish to thank Dennis Heuven for his contribution to the initial phases of the Cocoon implementation.

My acknowledgments should undoubtedly include Nicole Baveld, Thelma Nordholt, Marlous Weghorst, and Ellen van Erven. Profound thanks for their keen help in administrative issues, they merit all the best.

Thanks to all wonderful people I met in Twente for making me feel at home. Especially, as a board member of the Turkish Student Association at Twente, I had a lot of memories with my friends. In particular, my sincere thanks are due to Haktan Polattan, Ceren Xu Polattan, Erdi Aksoy, Çă̆rı Kızak, Koray Erdoğan, Muharrem Bayraktar, Devrim Yazan, and Umut İnal. I additionally convey my thanks to Şemsi E Kadir Akbaş, for helping me with settling in during my first year in Enschede.

I dedicate this thesis to my family who I love passionately.

To my parents, Gönül \& Serdar Türkeş_you are the prime reasons for all of my accomplishments. There could be truly no adequate thanks for your endless love, support, and prayers. I am forever grateful to you. Also to my parents-in-law, Gülay \& Sedat Karakaş-I am deeply thankful for your constant support and prayers. To my lovely sister Handefor being beside me for a long time in Enschede, for the joyful moments during our journeys, and most importantly for your abiding friendship.

Last, but first in my heart, to my dear wife Gözde-my breath of life, my other half. I owe my eternal gratitude for your enduring love, unwavering understanding, and unconditional support. From the very beginning, you have made my life perfect in many ways with your love. Thank you for our beautiful moments in these intense years. 


\section{Abstract}

Modern society is surrounded by an ample spectrum of personal mobile devices with short-range wireless communication support. This ubiquity creates an immense potential of new concepts for peoplecentric ad hoc networks that can be applied to every personal and social dimension of life. The last decade introduced the concept of Opportunistic Networks (OppNets) that facilitates delay-tolerant information sharing between mobile users anytime, anywhere, and every which way possible. OppNets constitute an appealing solution to provide connectivity in those situations where communication is desired, but situated network architectures fail to provide it effectively.

Despite the mobile revolution that the world is relishing today, the support of modern wireless technology in smart mobile devices is quite limited to fulfill OppNet services. While having promising potentials, the current wireless standards (e.g. Wi-Fi, Bluetooth) have restricted or hidden support for ad hoc communications in mobile operating systems. So far, such limitations have stimulated little research efforts to devise an alternative solution for the realization of OppNets. Besides, these standards are designed to achieve ad hoc communications under stable connectivity, therefore cannot cope with the highlydynamic characteristics of OppNets. Intrinsically, OppNets rely on mobility of users to extend the dimension of communications over large distances. By and large, the mobility assistance greatly needed by OppNets requires innovative design considerations for the networks of smart mobile devices.

This thesis focuses on the design, implementation, and analysis of a novel OppNet architecture intended for smart mobile platforms. Named Cocoon (Community-oriented Context-aware Opportunistic Networking), this architecture assists the practical development of a wide range of OppNet applications offered for general public use. 
Cocoon integrates versatile and lightweight opportunistic communication methods with a new collection of applications which are freely accessible by any group of mobile users. The presented applications span a rich collection of applications, such as short message services in challenged environments, safety monitoring in vehicular environments, and data dissemination in several demanding scenarios. In order to carry out these applications, Cocoon introduces a versatile and lightweight connectivity scheme, called opportunistic beacons, that expedites rapid and energy-efficient information sharing between smart mobile devices without requiring connections and sophisticated configurations. The design of opportunistic beacons is generic, so that it is readily integrable on top of the commonly-used wireless interfaces such as $\mathrm{Wi}-\mathrm{Fi}$ and Bluetooth.

The Cocoon architecture employs opportunistic beacons in the design and management of networking and application services. As a networking service, Opportunistic Beacon Networking (OBN) is introduced. Within OBN, a forwarding protocol is proposed and validated with extensive real-world experiments. The protocol is mainly offered for data dissemination purposes, but its end-to-end multi-hop routing performance has been evaluated as well. Furthermore, several improvements are presented, implemented, and compared for this protocol. For application management, a new set of service requirements are defined in view of the ever-changing nature of OppNets. These requirements are used in a distributed decision-making algorithm running alongside with OBN. The basic aim of the algorithm is to provide a quality-of-service to participating users by scheduling applications on their affiliated devices.

The OppNet applications presented in this thesis are quite promising considering their performance outcomes. To effortlessly and efficiently develop such applications, the Cocoon architecture can also be used as a development platform to realize opportunistic communications. To this respect, this thesis further provides an application programming interface guide on Android (Cocoon API) and a verified simulator on MATLAB/Octave that can be used to develop and analyze Cocoon-based networks. 


\section{SAMENVATting}

Onze moderne maatschappij is vergeven van kleine persoonlijke mobiele apparaten die voorzien zijn van radio's voor communicatie over korte afstand. Nieuwe concepten voor ad hoc netwerken waarbij de mens centraal staat worden mogelijk gemaakt omdat deze apparaten alom tegenwoordig zijn. De opkomst van Opportunistische Netwerken (OppNets) waarbij gebruikers op elke mogelijke manier informatie kunnen uitwisselen maakt dit duidelijk. OppNets vormen een aantrekkelijke oplossing voor communicatie in situaties waar conventionele communicatiemiddelen niet meer voldoen of afwezig zijn.

Ondanks de huidige mobiele revolutie is de ondersteuning van de in slimme mobiele apparatuur aanwezige moderne draadloze technologie voor OppNets zeer beperkt. Hoewel standaarden als Wi-Fi en Bluetooth in potentie ad hoc netwerken zouden kunnen ondersteunen doen ze dit niet of in zeer beperkte mate. Tot nu toe heeft dit nauwelijks geleid tot onderzoek naar alternatieve oplossingen voor de realisatie van opportunistische netwerken in, bijvoorbeeld, mobiele telefoons. De genoemde standaarden zijn ontworpen voor ad hoc netwerken waarbij verbindingen stabiel zijn, maar ze zijn ongeschikt voor OppNets met veelal zeer-dynamische verbindingen. OppNets zijn zelfs in belangrijke mate afhankelijk van de mobiliteit van gebruikers om informatieuitwisseling over grote afstanden mogelijk te maken.

Dit proefschrift concentreerd zich op het ontwerp, de implementatie en de analyse van een nieuwe en innovatieve OppNet architectuur voor slimme mobiele apparatuur. Deze architectuur, Cocoon (Community-oriented Context-aware Opportunistic Networking) ondersteunt de practische ontwikkeling van een breed scala aan publieke OppNet toepassingen. 
Cocoon verenigt een veelzijdige en lichtgewicht opportunistische communicatie met vrij toegankelijke toepassingen voor groepen mobiele gebruikers. De voorbeelden van mogelijke toepassingen in dit proefschift beslaan een breed spectrum, zoals een SMS service, een monitoring toepassing voor auto's en disseminatie van informatie onder moeilijke omstandigheden. Cocoon introduceert een systeem van lichtgewicht en veelzijdige opportunische communicatie, genaamd opportunistic beacons, waarmee snelle en energie-efficiente uitwisseling van informatie mogelijk wordt gemaakt zonder ingewikkelde configuratie vooraf. Het ontwerp van opportunistic beacons is zodanig generiek dat voor de onderliggende communicatie gebruik kan worden gemaakt van bestaande draadloze technieken, zoals Wi-Fi en Bluetooth.

De Cocoon architectuur maakt gebruik van de techniek van opportunistic beacons bij het ontwerp en management van netwerk- en applicatieservices. Een netwerkservice Opportunistic Beacon Networking (OBN) wordt in dit proefschrift geintroduceerd en uitgebreide realistische experimenten bevestigen de geldigheid. Hoewel OBN in eerste instantie bedoeld is voor disseminatie van informatie wordt ook de werking als "multi-hop" routeringsprotocol geevalueerd. Tevens worden verschillende verbeteringen beschreven, geimplementeerd en vergeleken met OBN. Naast OBN wordt een applicatiemanagement gedefinieerd specifiek voor het dynamische karakter van OppNets, resulterend in een gedistribueerd algorithme. Het doel ervan is een "quality-of-service" voorziening wanneer meerdere applicaties tegelijk gebruik maken van Cocoon.

De OppNet toepassingen beschreven in dit proefschrift zijn veelbelovend qua snelheid en prestatie. Cocoon is geschikt als ontwikkelplatform voor dergelijke applicaties en ondersteunt dit verder door te voorzien in een "application programming interface" voor Android (Cocoon API) en een simulator voor MATLAB/Octave. 


\section{ÖzET}

Çağdaş toplum, geniş sayıdaki kısa menzilli kablosuz iletişim destekli kişisel mobil cihazlarla donanmış durumdadır. Bu her yerde bulunan yaygınlık, insan odaklı tasarsız ağlar için yeni kavramların kişisel hayatta ve toplumsal boyutta uygulanmasını olası kılmaktadır. Geçtiğimiz on yıllık süreçte, mobil kullanıcıların kendi aralarında istediği zaman, istediği yerde, ve istediği doğrultuda gecikme toleranslı iletişimine olanak tanıyan Fırsatçı A ğlar kavramı ortaya çıkmıştır. Fırsatçı Ağlar, iletişimin gerekli olduğu, ancak yerleşik ağ mimarilerinin bu hizmeti etkin bir şekilde sağlamakta sorun yaşadığı durumlarda geçici bağlantılar sağlamak için cazip bir çözüm teşkil etmektedir.

Bugün dünyanın severek tanıklık ettiği mobil devrime rağmen, akıllı mobil cihazlarda bulunan günümüz kablosuz teknolojileri Fırsatçı A ğ servislerini sağlamak için oldukça yetersiz kalmaktadır. Mevcut kablosuz ağ standartları (Wi-Fi ve Bluetooth gibi), umut verici potansiyellerine karşın, mobil işletim sistemleri tarafından kısıtlanmış veya gizlenmiş tasarsız ağ desteğiyle sunulmaktadır. Bu tür sınırlamalar Fırsatçı Ağların gerçekleştirilmesi için bugüne kadar çok az sayıda alternatif çözümün araştırılmasına ve tasarlanmasına neden olmuştur. Bunun yanı sıra, bu standartlar tasarsız ă̆ iletişimini ancak istikrarlı bağlantısallık altında yerine getirebilmektedir; bu yüzden Fırsatçı Ağların devingen özelliğiyle başa çıkamamaktadır. Zira, Fırsatçı Ağlar iletişimi geniş mesafelere yaymak için özünde kullanıcıların hareketliliğine dayanmaktadır. Genel olarak, Fırsatçı Ağlar tarafından ihtiyaç duyulan hareket desteği akıllı mobil cihazlar ile oluşturulacak ağlar için yenilikçi tasarım anlayışları gerektirmektedir.

Bu tez, akıllı mobil platformlar için özgün bir Fırsatçı A ̆g mimarisinin tasarımı, gerçeklenmesi ve çözümlemesine odaklanmaktadır. Cocoon (Koza: Toplum-odaklı Bağlam-bilinçli Fırsatçı A $\breve{g}$ ) adı verilen bu mimari, kamu kullanımı için çok çeşitli amaçlara uygun Fırsatçı A $\breve{g}$ uygulamalarının geliştirilmesine yardımcı olmaktadır. 
Cocoon, her tür mobil kullanıcı tarafından kullanılmaya açık yeni kablosuz ağ uygulaması türlerini becerikli (çok yönlü) ve kılgısal (pratik) fırsatçı iletişim yöntemleriyle birleştirmektedir. Bu uygulamalar afet bölgelerinde kısa mesaj servisi sağlama, yaya ve taşıt trafiğine açık alanlarda emniyet takibi, zorlu (kritik) senaryolarda veri yayma gibi türlü gereksinimler için sunulmuştur. Cocoon, bu uygulamaları yürütmek için fırsatçı işaretçi olarak adlandırılan hafif ve çok amaçlı bir kablosuz bağlantısallık yöntemi takdim etmektedir. Fırsatçı işaretçiler, bilginin akıllı mobil cihazlar ile çabuk ve enerji verimli paylaş1mını bağlantı ve yapılandırma gerektirmeden kolaylaştırmaktadır. Genel bir tasarıma sahip fırsatçı işaretçiler Wi-Fi ve Bluetooth gibi yaygın kullanımlı kablosuz ăg arayüzlerinin üzerinde dolaysız olarak değerlendirilebilmektedir.

Cocoon mimarisi, fırsatçı işaretçileri ilgili ağ ve uygulama servislerinin tasarım ve yönetiminde kullanmaktadır. Mimari içerisinde bir ağ servisi olarak Firsatçı İşaretçi A $\breve{g} \imath$ sunulmuştur. Fursatçı İşaretçi A $\breve{1}$ bünyesinde bir iletme protokolü önerilmiş ve ayrıntılı gerçek hayat deneyleriyle geçerliliği doğrulanmıştır. Bu protokol esas olarak veri yayma amaçları için önerilmiş, fakat noktadan noktaya çok atlamalı iletim başarımı da ölçülmüştür. Ayrıca, bu protokol için birkaç iyileştirme yöntemi sunulmuş, gerçeklenmiş, ve başarımları karşılaştırılmıştır. Uygulama yönetimi için, Fırsatçı Ağların sürekli değişen doğası göz önünde tutularak yeni servis gereksinimleri tanımlanmıştır. Bu gereksinimler, Fursatçı İşaretçi Ağı ile birlikte çalışan bir dağıtık karar verme algoritması için kullanılmaktadır. Bu algoritmanın amacı, kullanıcıların cihazlarında çalışan uygulamaların zaman planlamasını yaparak ağda servis kalitesini sağlamaktır.

Başarım sonuçları itibariyle, bu tezde sunulan Fırsatçı A $\breve{g}$ uygulamalarının oldukça umut verici olduğu gösterilmiştir. Bu tür uygulamaları çaba harcamadan ve etkin bir biçimde geliştirmek için, Cocoon mimarisi bir geliştirme platformu olarak da kullanılabilmektedir. Bu nedenle, tez içeriğinde Android sistemler için geliştirilmiş bir uygulama programlama arayüzü (Cocoon API) ve Cocoon temelli ağların yaratılmasına ve incelenmesine yarayan MATLAB/Octave ile uyumlu, tasarımı doğrulanmış, bir simülator sunulmuştur. 


\section{Contents}

1 INTRODUCTION $\quad 1$

1.1 The MobIle Freedom . . . . . . . . . . . . . . . . . . . . 2

1.1.1 Mobile Ad Hoc Networks . . . . . . . . . . . . . . . . . . 3

1.1.2 Delay Tolerant Networks . . . . . . . . . . . . . . . 4

1.2 OPPORTUNISTIC NetWorKS . . . . . . . . . . . . . . . . . . . . . . 8

1.2.1 SYSTEM CHARACTERISTICS . . . . . . . . . . . . . . . 13

1.2.2 SYSTEM REQUIREMENTS . . . . . . . . . . . . . . . . . . . . . . . . . . . . . 14

1.3 THESIS SCOPE . . . . . . . . . . . . . . . . . . . . . . . . . 15

1.3.1 ReSEARCH MotivATION . . . . . . . . . . . . . . . . . . . . 15

1.3 .2 ReseARCh ОвJеCtIVES . . . . . . . . . . . . . . . . . . 16

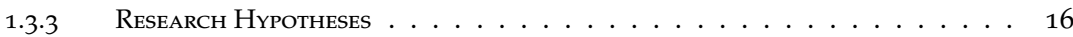

1.3 .4 RESEARCH APPROACH . . . . . . . . . . . . . . . . . 17

1.4 Thesis Contributions . . . . . . . . . . . . . . . . . . . . . . . . 18

1.5 THESIS ORGANIZATION ........................... 20

2 StAte-of-The-Art $\quad 23$

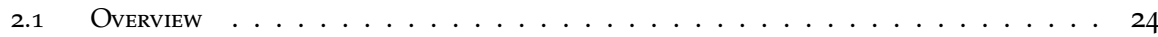

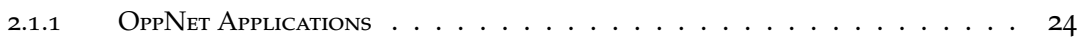

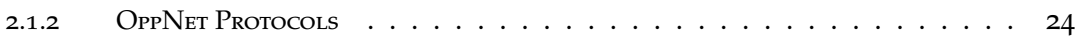

2.1.3 Chapter Organization . . . . . . . . . . . . . . . . . 25

2.2 Ad Hoc Networking Technologies in Smart Mobile Platforms . . . . . . . . . . . . 26

2.2.1 General Characteristics . . . . . . . . . . . . . . . . . 27

2.2.2 Challenges regarding OppNet Characteristics . . . . . . . . . . . . . . 28

2.2.3 Mobile Operating SYSTEM Support . . . . . . . . . . . . . . . . . . 31

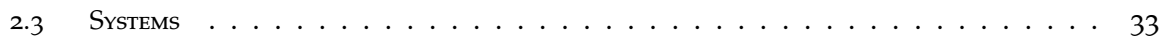

2.3.1 Network Architectures . . . . . . . . . . . . . . . . . 33

2.3.2 Connectivity Schemes . . . . . . . . . . . . . . . . . . 35

2.3 .3 APPLICATIONS \& SERVICES . . . . . . . . . . . . . . . 36

2.3.4 BeAcon Profiles . . . . . . . . . . . . . . . . . . 36

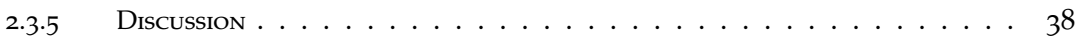

2.4 ForWARdING Protocols . . . . . . . . . . . . . . . . . . . . 41

2.5 Concluding Remarks ． . . . . . . . . . . . . . . . . . . . . . . . . . . . . . 45 
3 Community-Oriented Context-Aware

Opportunistic Networking Architecture 47

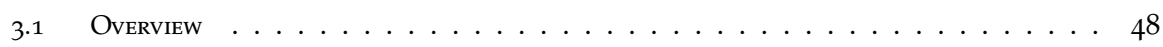

$3.1 .1 \quad$ SYStem Requirements $\ldots \ldots \ldots \ldots \ldots \ldots \ldots \ldots \ldots \ldots \ldots$

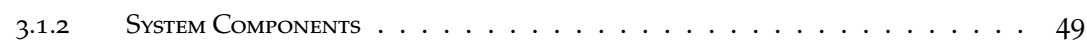

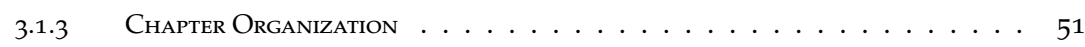

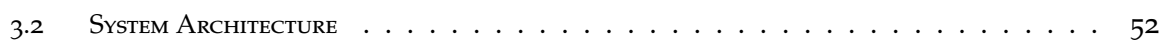

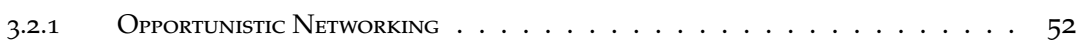

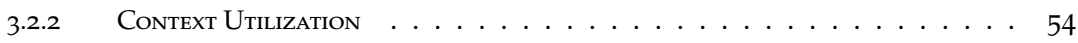

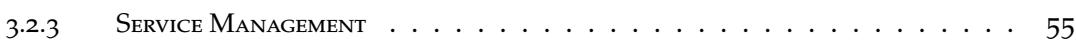

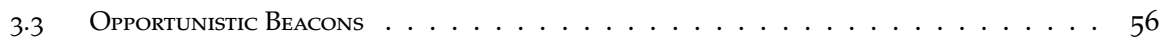

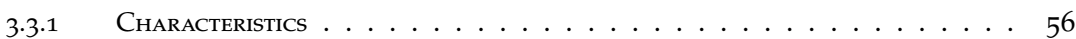

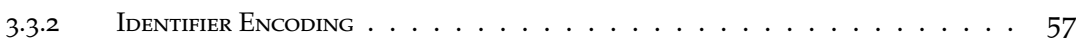

$3.3 .3 \quad$ APPLICABILITY STUDY $\ldots \ldots \ldots \ldots \ldots \ldots \ldots$

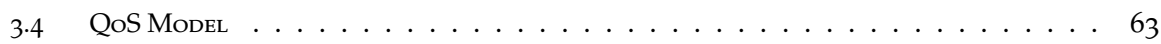

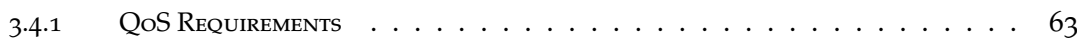

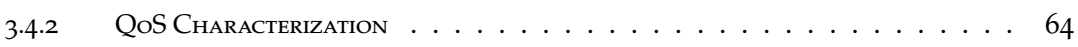

3.5 Concluding Remarks $\ldots \ldots \ldots \ldots \ldots \ldots \ldots \ldots \ldots$

4 Opportunistic Beacon Networking $\quad 69$

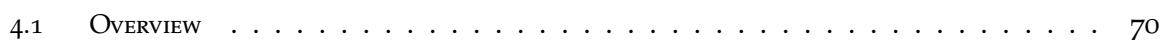

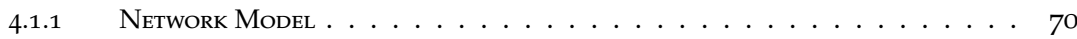

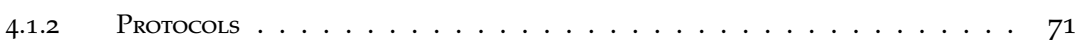

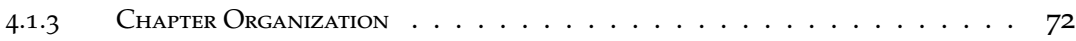

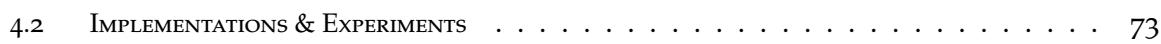

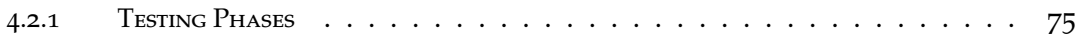

4.2.2 Model Evaluation Parameters $\ldots \ldots \ldots \ldots \ldots \ldots \ldots$

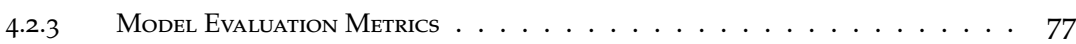

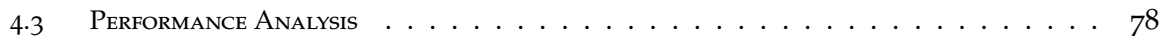

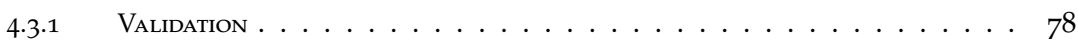

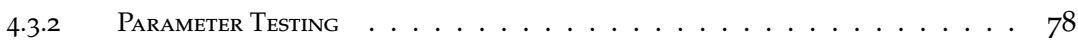

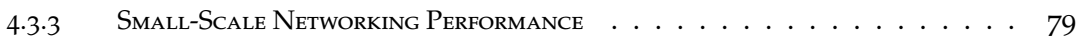

4.3.4 Large-Scale Networking Performance . . . . . . . . . . . . . . 80

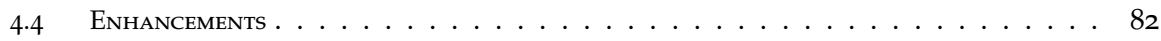

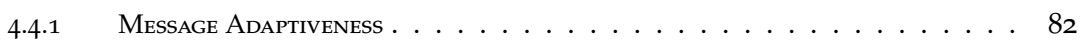

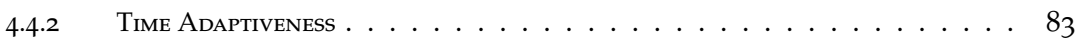

4.4 .3 Dual Radio Utilization . . . . . . . . . . . . . . . . . . . . . . . . . . . . . . . . . . . . . . . . . . . . . . .

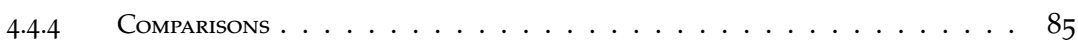

4.5 Concluding Remarks $\ldots \ldots \ldots \ldots \ldots \ldots \ldots$ 
5 Applications $\quad 91$

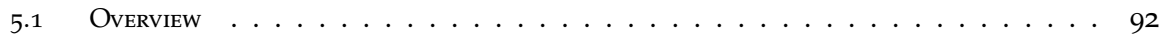

5.1.1 Data Dissemination ApPlichtions . . . . . . . . . . . . . . . . . 92

5.1 .2 Data Routing Applications . . . . . . . . . . . . . . . . . 92

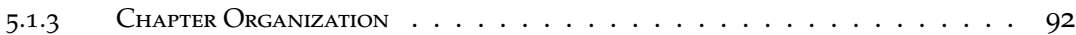

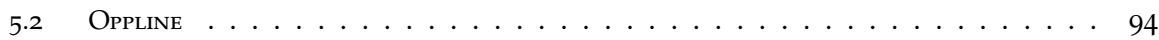

5.2.1 COMMUNiCATION MODEL . . . . . . . . . . . . . . . . 95

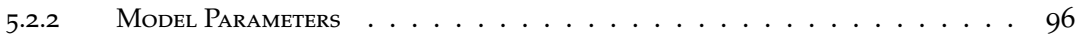

5.2 .3 MesSAGE ENCODING . . . . . . . . . . . . . . . . . 97

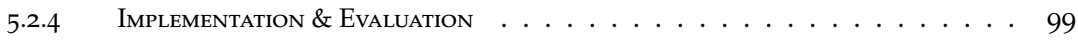

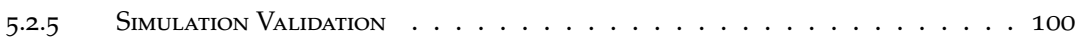

5.2.6 Test Setups \& Model Evaluation Parameters ～. . . . . . . . . . . . . . . . 101

5.2.7 Evaluation Metrics . . . . . . . . . . . . . . . . . . . . . . . 101

5.2.8 Performance AnAlysis . . . . . . . . . . . . . . . . 102

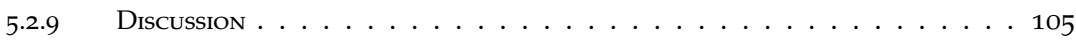

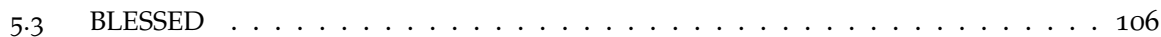

5.3 .1 Communication Model . . . . . . . . . . . . . . . 106

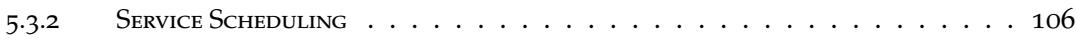

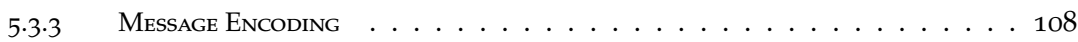

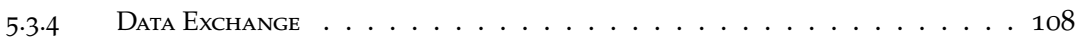

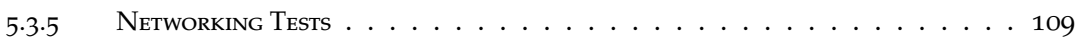

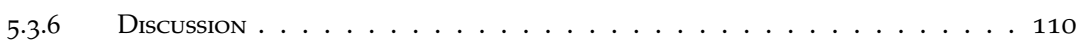

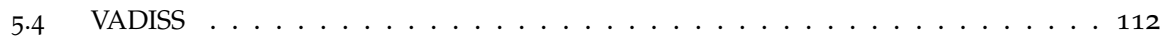

5.4 .1 DisSeminAtion Protocol . . . . . . . . . . . . . . . . 113

5.4.2 PARticipatory RoAd TRAFic Monitoring . . . . . . . . . . . . . . . . . 114

5.4 .3 DATA DisSeminATION . . . . . . . . . . . . . . 116

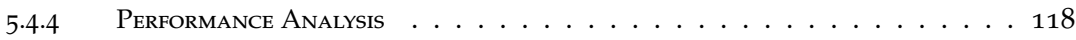

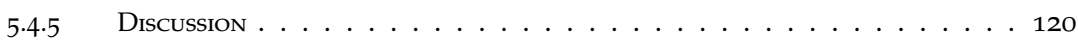

5.5 Concluding Remarks . . . . . . . . . . . . . . . . . . . . . . . . . . . 121

6 Conclusions \& Future Prospects 123

6.1 Reflections \& IMPLicAtions . . . . . . . . . . . . . . . . . . . . . . . . . . . . 124

6.1.1 Contributions ReVISITED . . . . . . . . . . . . . . . . . . . . . . . 124

6.1.2 ReseArch Questions ANSWERED . . . . . . . . . . . . . . . . . . . . 126

6.1.3 LeSSONS LEARNED . . . . . . . . . . . . . . . . . . . . . . . 128

6.2 Open Research Directions . . . . . . . . . . . . . . . . . . . . . . . . . . . . . . . 129

6.2.1 Brofdening the Context . . . . . . . . . . . . . . . . . . . 129

6.2 .2 INFRASTRUCTURE . . . . . . . . . . . . . . . . . . . . . 129

6.2.3 CoMplex SCENARIOS . . . . . . . . . . . . . . . . . . . . . . . . . . 129

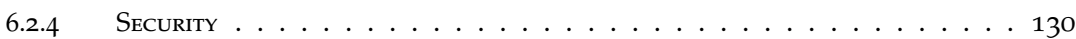

6.2.5 The Future of BeAcons . . . . . . . . . . . . . . . . . 130

6.3 Final ReMARKS . . . . . . . . . . . . . . . . . . . . . 131 
$\begin{array}{lr}\text { A Acronyms \& Notations } & 141\end{array}$

B Oppliqué • API Guide 145

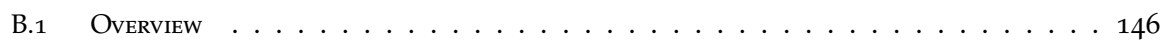

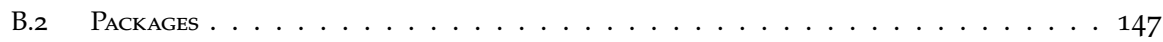

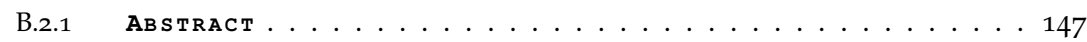

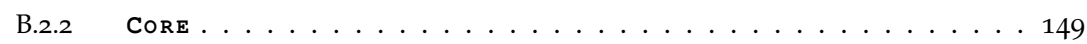

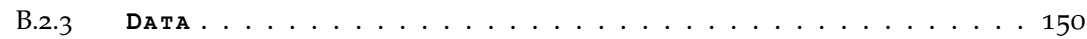

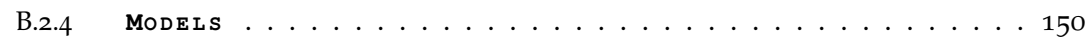

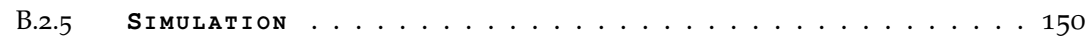

B.3 Matn Structures . . . . . . . . . . . . . . . . . . . . . . . . . 151

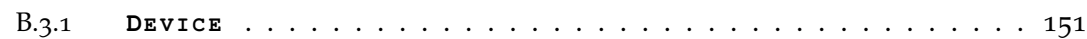

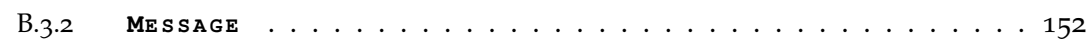

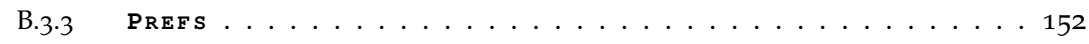

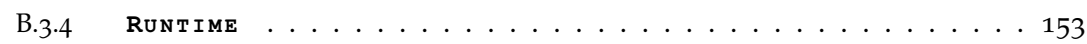

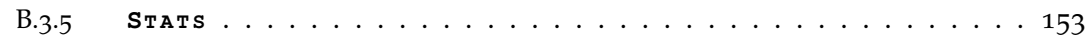

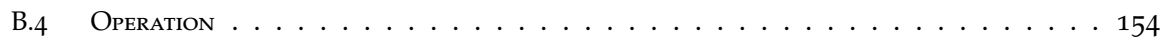

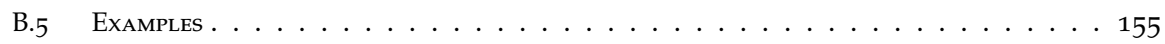

$\begin{array}{lr}\text { C Cocoon • API Guide } & 157\end{array}$

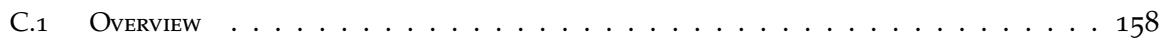

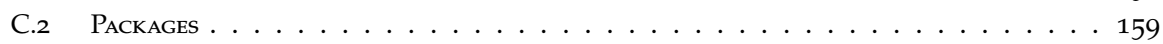

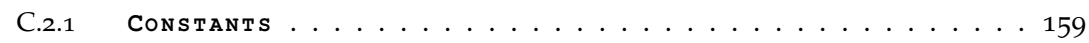

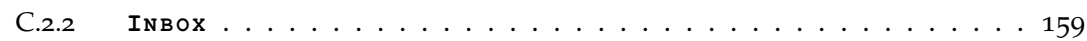

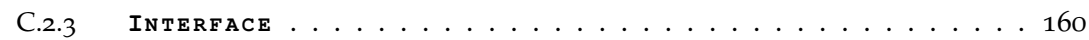

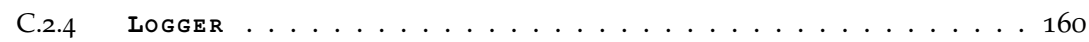

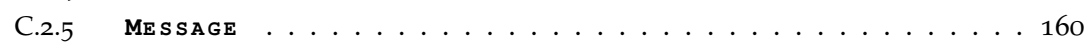

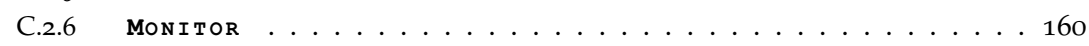

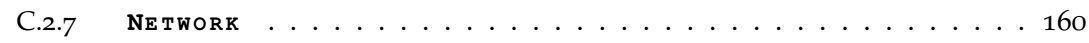

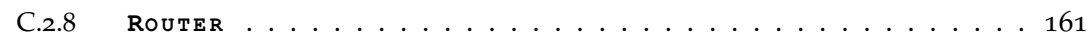

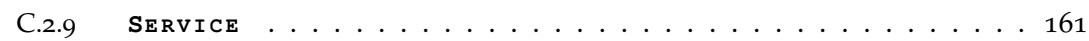

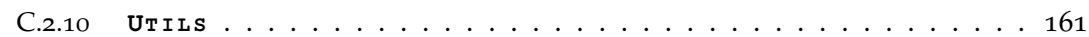

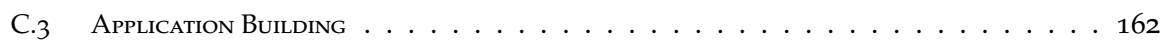

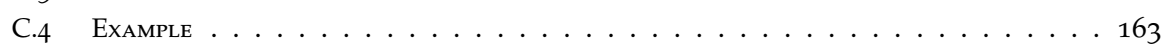

$\begin{array}{ll}\text { INDEX } & 166\end{array}$

$\begin{array}{lr}\text { Aвout the Author } & 169\end{array}$ 

CHAPTER 1

\section{INTRODUCTION}

COMMUNICATIONS IN CONTEMPORARY LIFE IS BEING RESTRUCTURED WITH THE RISE OF WIRELESS-NETWORKED SOCIETY. THE LAST DECADE HAS INTRODUCED Opportunistic Networks (OpPNets) Which enAble information SHARING THROUGH OCCASIONAL PEER-TO-PEER CONNECTION OPPORTUNITIES [1, 2]. COMPLEMENTARY TO, OR IN SUPPORT OF THE SITUATED COMMUNICATION SYSTEMS, OPPNETS PROVIDE DELAY-TOLERANT AD HOC COMMUNICATIONS UNDER HIGHLY-DYNAMIC ROUTING CONDITIONS. IN THE REALM OF AD HOC NETWORKS, ONE CURRENT TREND IS TO FORM OPPNETS WITH THE EXPLOITATION OF SMART MOBILE DEVICES USED BY PEOPLE. SUCH DEVICES WITH WIRELESS LOCAL AREA NETWORK (WLAN) AND PERSONAL AREA NETWORK (WPAN) SUPPORT ARE INCREASINGLY BEING ADOPTED IN DAILY LIFE, FORMING A HIGH POTENTIAL OF INTERCONNECTEDNESS IN PUBLIC SPACE. OPPNETS OFFER DISTINCTLY ATTRACTIVE ENABLING TECHNOLOGIES FOR PEOPLE-CENTRIC PERVASIVE ENVIRONMENTS.

THIS THESIS PRESENTS THE DESIGN, IMPLEMENTATION, AND ANALYSIS OF aN OppNet architecture Which provides Lightweight aNd Versatile AD HOC SHORT MESSAGE COMMUNICATIONS WITH MODERN MOBILE DEVICES. NAMED COCOON, THE ARCHITECTURE RELIES ON THE CONCEPT OF SMART WIRELESS BEACONS THAT ARE DESIGNED TO SUPPORT OPPORTUNISTIC INFORMATION SHARING WITH MOBILITY ASSISTANCE.

Providing A BRIEF OVERVIEW OF THE ADVANCING MOBILE COMMUNICATION TECHNOLOGIES, THIS CHAPTER INTRODUCES OpPNETS, EXPLAINS THEIR CHALLENGES AND OPPORTUNITIES, AND PRESENTS THEIR APPLICATION AREAS. Regarding COMMUNITY-ORIENTED OpPNeTS, THIS CHAPTER ADDITIONALLY IDENTIFIES OUR RESEARCH QUESTIONS FOCUSED IN THE THESIS, PRESENTS OUR RESEARCH APPROACH, OUTLINES THE CONTRIBUTIONS PRESENTED THROUGHOUT THE DISSERTATION, AND FINALLY CONCLUDES WITH THE THESIS ORGANIZATION. 


\subsection{The Mobile Freedom}

Information is mobile by its very nature. As to fulfill the purpose of communications, it is always on the move from one place to another. For the first time in the history, it is also mobileaccessible since the turn of this century. Owing to the advancements in wireless technologies, the humanity enjoys the freedom of sharing information on the move with the use of mobile communication devices.

Mobile communications has rapidly become an inseparable part of daily life, not only of our private world but also of our social world. The ability to communicate on-the-move has profoundly refashioned the modern lifestyle in a way never seen before: It has revolutionized the way of our thinking, working, relating to people, interaction with the world, searching for relevant information, so on. From a broader perspective, it has provided a higher level of flexibility in information sharing, leading to a significant migration from wired to wireless networks [3], and raised user expectations for increased connectivity. These trends have incited the evolution of mobile communication devices into smart mobile devices which are equipped with powerful computational units, physical sensors, enhanced cellular data connection and multiple short-range wireless interfaces. Smart mobile devices have provided high versatility in terms of information sharing as well as high connectedness for users to easily maintain and develop social ties.

Today, smart mobile devices such as smartphones, tablet and laptop PCs, and smartwatches are considered as ubiquitous computing platforms not only for their increased computational and connectivity capabilities, but also for their daily usage [4]. The worldwide smart mobile device usage rapidly reached a maturity [5], which clearly points out that smart mobile devices already achieved a successful penetration to the customer market within less than a decade. The mobile revolution is turning into an achievement through not only the high number of smart mobile devices, but also of other connected device types such as mobile feature phones with wireless network access support, machine-to-machine (M2M) communication devices, and consumer electronics with short-range wireless access support. Figure 1.1 shows the Ericsson's 2015 Mobility Report on the current and estimated number of connected devices worldwide. Among all, mobile phones (including smart ones) have been the largest category of connected devices [6]. This year, the number of mobile phones in the world will surpass the world population for the first time, as the analytic report presented in [7] also confirms. Besides, the number of M2M devices is expected to grow at an annual growth rate of $25 \%$ within the next decade, driven by disruptive use cases. In total, around 27 billion connected devices are expected by 2021, of which around 12 billion will be handheld devices whereas around 15 billion will be M2M and consumer electronics devices.

The high proliferation of the above-mentioned mobile device types together with the widely-available situated communication systems form ubiquity in terms of being connected anytime and anywhere. Mobile devices can form connections between each other through centralized infrastructures such as cellular base stations and wireless access points (APs). Nevertheless, in challenging scenarios, it may take time to set up the infrastructure-based network 

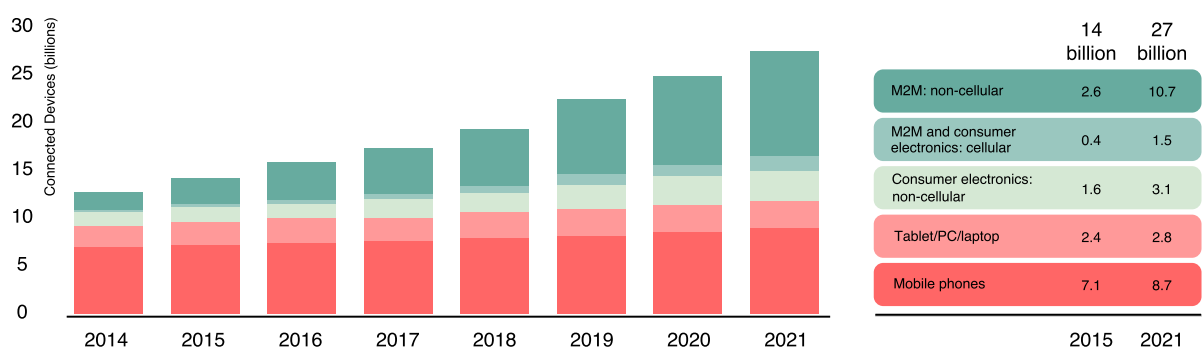

Ericsson's forecast on the number of connected devices in the world.

Examples of consumer electronics devices: smart TVs, digital media boxes, gaming consoles, etc.

Examples of M2M (Machine-to-machine): connected cars, utility meters, remote metering, etc.

while the deployment costs to install an infrastructure can be quite high [8]. Alternatively, thanks to the technical progress achieved recently, majority of the commercially-off-the-shelf (COTS) mobile devices are also capable of establishing more specialized networks that can operate in a particular locality without relying on an infrastructure, i.e. they can communicate in ad hoc fashion $[9,10]$. In general, this decentralized type of communications is referred to as Ad Hoc Networks [11], and regarding mobile devices, one specific type of it is called Mobile Ad Hoc Networks.

\subsubsection{Mobile Ad Hoc Networks}

A Mobile Ad Hoc Network (MANET) is a rapidly-deployable, dynamically self-configuring communication architecture comprising mobile devices that can connect to each other while moving arbitrarily inside a designated networking space [12]. Each device participating in a MANET can act as a source, as a destination, and as a router of different information at the same time. Regardless of geographic location, MANETs provide an increased information sharing flexibility in a multi-hop fashion in comparison to the other types of wired and wireless networking systems.

A MANET is contingent upon topological changes over time due to device mobility. Each device in a MANET is free to move independently in any direction, and will therefore change its links to other devices frequently. Additionally, new devices may emerge to join the network whereas existing devices may vanish at any time. As a result, a MANET is subject to fluctuations in wireless network conditions, and is therefore often prone to link failures. In this regard, routing in a MANET architecture has to automatically recover itself from different types of faults or negative changes whenever possible [13]. On the other hand, MANET routing necessitates end-to-end connectivity between a source and a destination that is always present $[12,13]$. MANETs are hereupon restricted to a local area of wireless devices to facilitate the identification or collection of certain phenomena in a local area for a variety of applications such as air pollution monitoring, disaster early warning systems, indsutrial/structural monitoring, transport and logistics, and healthcare. 
Different types of architectures can be used for such applications on condition that transmission routes are available at all times. Given this prerequisite, MANET routing protocols can employ different strategies that are mainly classified in three categories:

- Reactive routing: Routes are set up based on demand by flooding a query from source to destination. The advantage of reactive routing is that it does not use bandwidth except when running a routing query. During query processes, however, network overhead may increase due to flooding process.

- Proactive routing: Routes are established based on continuous route maintenance. The advantage of proactive routing over reactive routing is that routes are always known (table-driven) by participating devices. However, a constant overhead is present to control the route information.

- Hybrid routing: The combination of reactive and proactive routing strategies.

Dynamicity is one of the fundamental challenges of MANETs. Due to continuous route reconfigurations, MANETs are power-costly [14]. Although scalability issues can be overcome with effective traffic control mechanisms, expandability remains as a big concern in MANETs. Besides, routing in MANETs presumes disruptions as failures, therefore, is badly affected by frequent disconnections. As a known fact, MANETs are unable to go beyond network flexibility since they require a connected network [13].

As rather more flexible networking concept, the idea of Delay-Tolerant Networking emerged to support communications in between disjoint network topologies by accommodating long disruptions and delays between and within those networks.

\subsubsection{Delay Tolerant Networks}

A Delay-Tolerant Network (DTN) differs from the MANET concept in which the end-to-end connectivity constraint is released [15]. Consequently, routing in DTNs are disruption- and delay-tolerant to support multi-hop transmissions in case of disconnections. In order to handle disconnections, a special routing method, called store-carry-forward, is derived [16]. As illustrated in Figure 1.2, once the information is created or received by a device, it is carried until a connection is established with another device, then is forwarded to the other side of the connection. Unlike traditional routing protocols, DTN routing protocols have high transmission delays on the condition of long disconnection periods. The destination may receive the information from the source if the mobility helps, otherwise the information may not be received at all. In brief, DTNs relax the constraints on connectivity, but transmissions rely on contingency of either deterministic or stochastic contacts. As shown in Figure 1.3, considering a successful transmission between a source device (shown as $S$ ) and a destination device (shown as $D$ ), the number of relay devices (shown as $n$ ) and the contacts for each relay device (shown as $r_{i}$ ) may vary. With respect to this, the main goal in DTN routing is based on two basic objectives: 
- To maximize the delivery probability. Given a contact $\left(r_{i}, r_{j}\right)$, let $p_{i}$ denote the relay probability of a particular network packet from $r_{i}$ to $r_{j}$ occurring at a specific time. The end-to-end delivery probability of a packet can be formulated as,

$$
\prod_{i=1}^{n} p_{i}
$$

which includes only the $p_{i}$ 's occurring between its source and destination.

- To minimize the delivery delay. Given a contact $\left(r_{i}, r_{j}\right)$, let $t_{i}$ denote the relay latency of a particular network packet from $r_{i}$ to $r_{j}$ until the contact becomes available. The end-to-end delivery latency of a packet can be formulated as,

$$
\sum_{i=1}^{n} t_{i}
$$

which includes only the $t_{i}$ 's occurring between its source and destination.

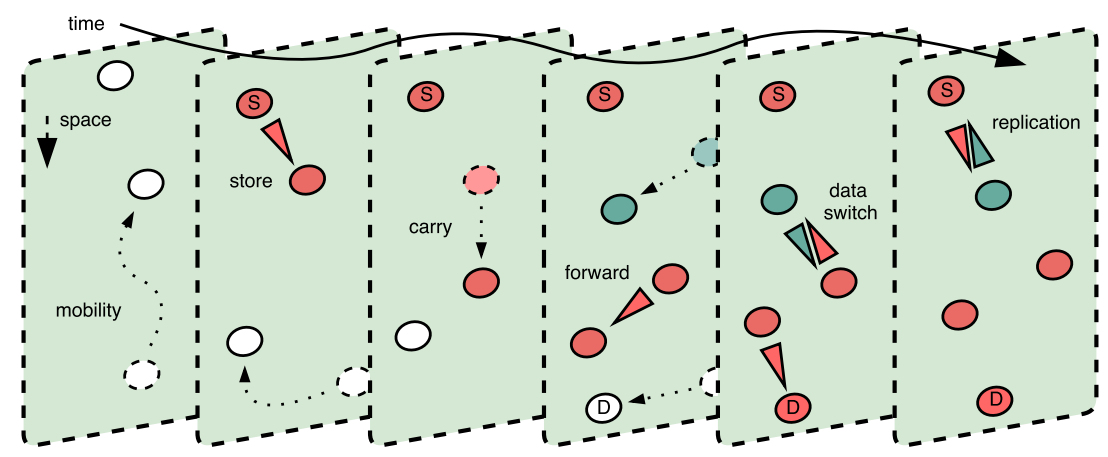

A DTN illustration

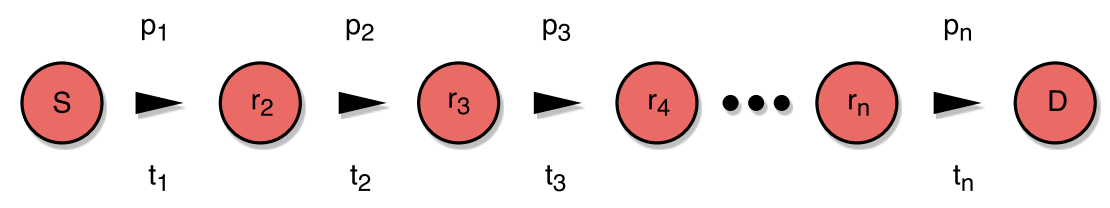

The intermittent links between a DTN source and destination 
In order to increase the delivery likelihood and to decrease the delivery latency of DTN packets, DTN routing protocols often use message replication [17]. In replication-based schemes, multiple copies of a network packet are relayed to multiple contacts. In consideration of resource optimization, selection of the most appropriate contacts for packet forwarding becomes the key target to avoid bandwidth contentions, to control congestions, and most importantly to increase DTN routing efficiency. Packet forwarding strategies may vary based on the contact types. Another strategy to provide resource optimization is to define a limit for packet lifetime. That is, DTN packets can be assigned time metrics such as Time-to-Live and Time-to-Send or information-specific metrics such as hop count and replication count to limit replication to a period of time or to a level of scalability $[18,19]$.

In DTNs, mobility is exploited as an enabler to broaden communication between devices scattered over large areas or to provide a temporary link between disjoint networks. However, mobility creates connectivity-related issues due to the volatile contacts between the devices. In case of immediate connectivity changes, transmission routes are exposed to change over time and space. Due to the fact that sudden topological variations may happen, DTN routing protocols make contact-based forwarding decisions instead of link-based forwarding decisions to eliminate incomplete traffic control [20, 21]. For this reason, MANET protocols are not applicable for DTNs since contemporaneous end-to-end connectivity is not always guaranteed [21].

Based on device mobility characteristics, a DTN deployment might have different contact types classified in three categories:

- Scheduled contacts: Contacts that are known before they occur. Contact time and contact duration are known beforehand with a high degree of precision.

e.g. message ferries such as buses, trams, and ferryboats.

- Predictable contacts: Contacts that can be predicted based on past observations. Contact time and contact duration can be estimated with some degree of determinism.

e.g. people with daily routines such as post officers.

- Opportunistic contacts: Contacts that are neither scheduled nor predicted, i.e. they are not known before they occur. Contact time and contact duration are stochastic.

e.g. people with random trajectories such as tourists strolling in a city.

The DTN concept extends the MANET concept towards highly dynamic, disjoint, and heterogeneous networks of devices to support challenged communications in highly unstructured topologies. In other words, DTNs are designed to be efficient for interconnecting highly heterogeneous networks even if end-to-end connectivity may never be available. Especially in people-centric DTN architectures, devices are often scattered into disjoint topologies. In addition, devices often communicate through opportunistic contacts, in which a sender and receiver make contact at an unscheduled time. 
From the DTN concept comprising opportunistic contacts, the concept of Opportunistic Networking emerged in the last decade. As a specialized implementation of the DTN principle, Opportunistic Networking comprises mobile devices to allow short messaging between people anytime and anywhere without requiring an infrastructure [1]. To this respect, Opportunistic Networking is solely considered in the context of people-centric ad hoc communications. Despite this distinction, Opportunistic Networking owns the same routing approach designed for DTNs, i.e. store-carry-forward approach coupled with the mobility of people. For that reason, Opportunistic Networking allows different types of short information to be spread through people by exploiting the distributed nature of them. This characteristic makes Opportunistic Networking attractive in cases where simple notifications rise in importance between any disconnected group of people in social space.

Opportunistic Networking represents a convenient channel for wireless communications where computing is pervasive through people, being seamlessly embedded in the fabric of sensor-enabled everyday devices. This vision was first articulated by Weiser in his description of ubiquitous computing [22]. Historically, this vision initially gave birth to Wireless Sensor Networks (WSNs) with proliferation of sensor technology in the last two decades [23]. WSNs employ distinctive routing approaches for pervasive communications through sensor devices which are equipped with resource-constrained components and low-power short-range radios. Traditionally, WSNs are deployed for monitoring applications based on low-rate data collection as well as for more complex operations ranging from target tracking to healthcare. WSNs aim to provide all-inclusive networking solution for various environments where a human presence is generally risky or even impossible. In Opportunistic Networking, on the other hand, human takes on the leading role to provide networking with everyday devices as they generally found on or close to unsettled public communication areas.

In this thesis, our main concentration is on Opportunistic Networking in the context of community-oriented communications. The next section elaborates on the unique characteristics, challenges, and application areas of Opportunistic Networks. 


\subsection{Opportunistic Networks}

In general terms, Opportunistic Networks (OppNets) are characterized as ad hoc and multihop networks in which the participating mobile devices self-organize into a set of disjoint networks without an infrastructure [1,2]. More specifically, with the exploitation of personal mobile devices for opportunistic means of communications, OppNets refer to the emerging trend of augmenting such devices with sensing, computing, and networking capabilities, connecting them in a social community to achieve a common communication goal in an ad hoc fashion. OppNets enable anytime and anywhere multi-hop information switching as the participating users are mobile in most cases. By this means, OppNets can be considered as networks of people. Recently, significance of social collaboration has gained currency in the domain of mobile ad hoc communications [24, 25], making OppNets built up with smart mobile devices a specific use case for differentiated networking solutions.

As previously mentioned, the ever-increasing availability of short-range wireless access interfaces with ad hoc support fitted in smart mobile devices (also in other connected device types) generates a high potential for communications by decentralized means. The most widely-used wireless local area networking (WLAN) and wireless personal area networking (WPAN) standards presented in smart mobile devices, respectively Wi-Fi and Bluetooth, can support inter-device communications with their newly-introduced peer-to-peer (P2P) protocols. Besides, the advancing features of physical sensors integrated in smart mobile devices create an availability in terms of information richness. Devices can create any kind of content to share with co-located devices. In parallel, computational units and memory/storage capabilities of smart mobile devices are getting powerful with each passing day, allowing any kind of shared content to be processed and analyzed on-the-fly. In view of these technological advances, OppNets are becoming extremely viable, cost-effective, and topology-flexible type of communications in comparison to the traditional mobile communications.

OppNets extend the scope of well-known application areas towards the public space by allowing smart mobile devices of people to directly get involved in the process of different networking services. More explicitly, people with different social status, interests, habits, and routines intersecting at various locations of interests can cooperatively exchange information by using their personal mobile devices, simply making contacts by opportunistic means of communications. Such transient contacts can serve for the development and enhancement of specific OppNet applications intended for society's end use. Apart from smart mobile devices, other types of connected devices such as M2M devices and consumer electronics used in public space can also serve for these applications to improve the level of connectedness.

While OppNets show an increased potential for various applications, they are certainly unsuitable for those which are tightly constrained by short time delays (e.g. multimedia streaming), or applications dependent on end-to-end transport connections. Thus, common examples of OppNet use-cases include distribution of short messages such as alerts, alarms, and simple notifications in urban areas, location-based services, and providing simple means of Internet access in rural/developing regions. 
Considering this shift from the host-centric networking to content-centric, OppNets are beneficial for those applications which aim at delay-tolerant connectivity between people in challenged and opportunistic scenarios.

OppNets cover a wide application scope which basically revolutionized the way classical ad hoc networks conduct their duties. Figures 1.4 illustrates a set of OppNet application scenarios that can overlap with each other in modern life. In the following, some examples of OppNet applications are briefly surveyed based on their area of usage:

- Challenged Environments: Challenged environments comprise those situations where communication is desired, but traditional networking systems completely or partially fail to provide it effectively. OppNets can be deployed in destroyed places as well as in rural and remote areas as an alternative to unavailable infrastructure-based communications. As an example, public awareness during disasters can be raised through an OppNet application running people's smartphones as a cheap way of network service. Thus, emergencies can be monitored or informed at any point and in a distributed manner by mobile-phone carriers.

As another example, groups of specialized people to perform specific tasks such as mining, mountain climbing can make use of OppNets to stay connected when they are out of global network coverage.

On the other hand, OppNets can be deployed to relieve well-covered situated systems by means of opportunistic data off-loading [26, 27]. Especially in highly-populated regions, situated systems are subject to decay or fail because of their inadequate services [28]. As the mobile data usage in the world grows exponentially [6], OppNets can reduce the burden of high data traffic regulated over the communication infrastructures. In case of infrastructure shut-downs, OppNets can serve as an alternative way of communications. For instance, at crowded events where cellular systems often fail, density and mobility of people can be exploited to opportunistically switch data between spatially-far people.

Furthermore, OppNets can be applied in place of the infrastructures which are unreliable, sporadic, expensive or censored. For instance, in a time when governments repressively monitor various types of communication in their country, their citizens might be in need for off-the-grid means of communications.

- Vehicular Environments: People in vehicular environments can form an opportunistic connectedness as a cost-free alternative for current traffic information systems with their ubiquitously present mobile phones for sensing, data handling, and information exchange. Such an OppNet application intends to increase awareness of drivers and pedestrians for critical cases such as bad road conditions and improper driver behaviour that may potentially cause accidents. As traffic monitoring data is collected and shared by hundreds and even thousands of traffic participants with their smart mobile devices, the resulting information on traffic conditions becomes always up-to-date and highly accurate. Such OppNet applications may also take the advantage of road-side units or other means of infrastructures in case of necessity. 
FIGURE 1.4

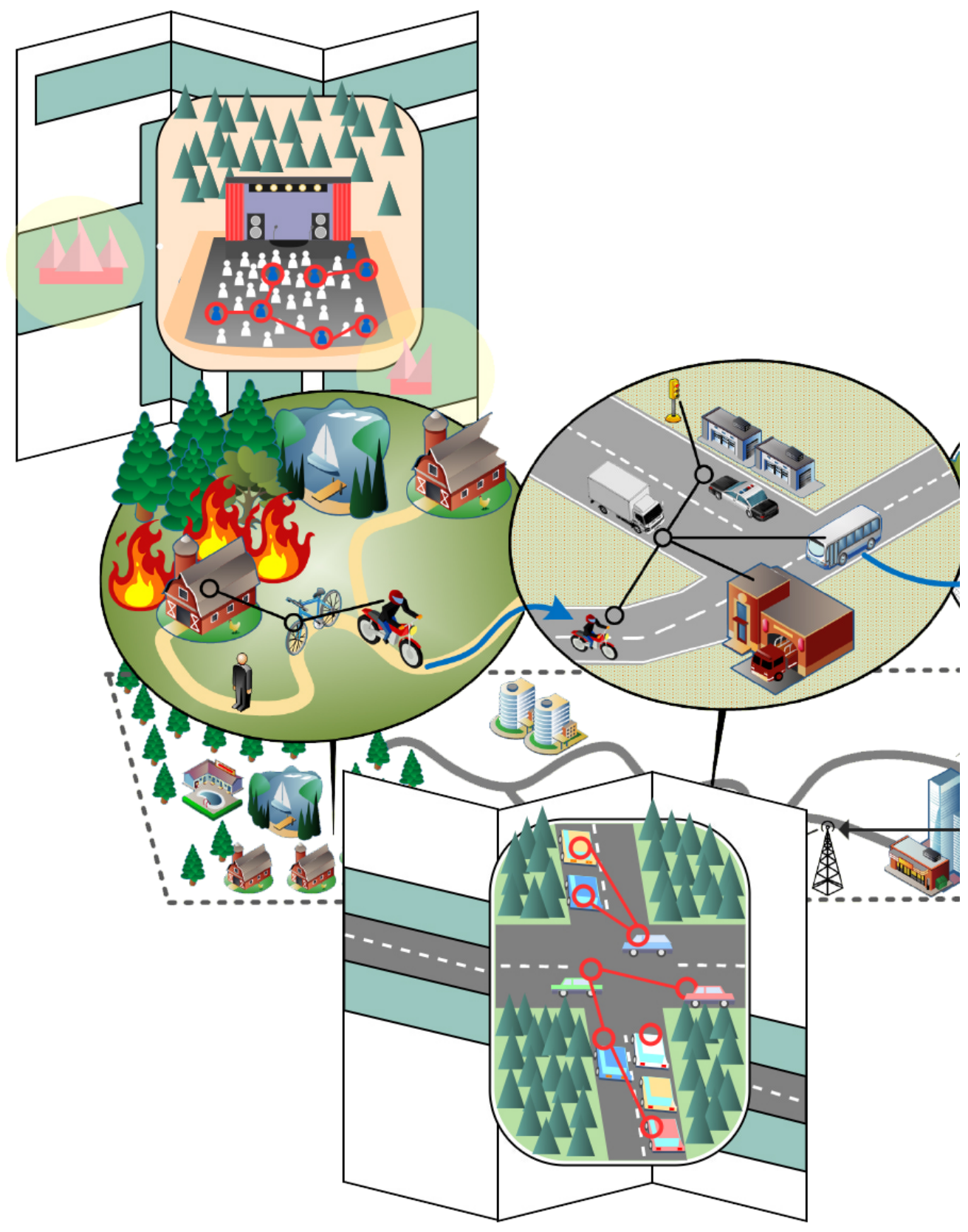

Application scenarios 


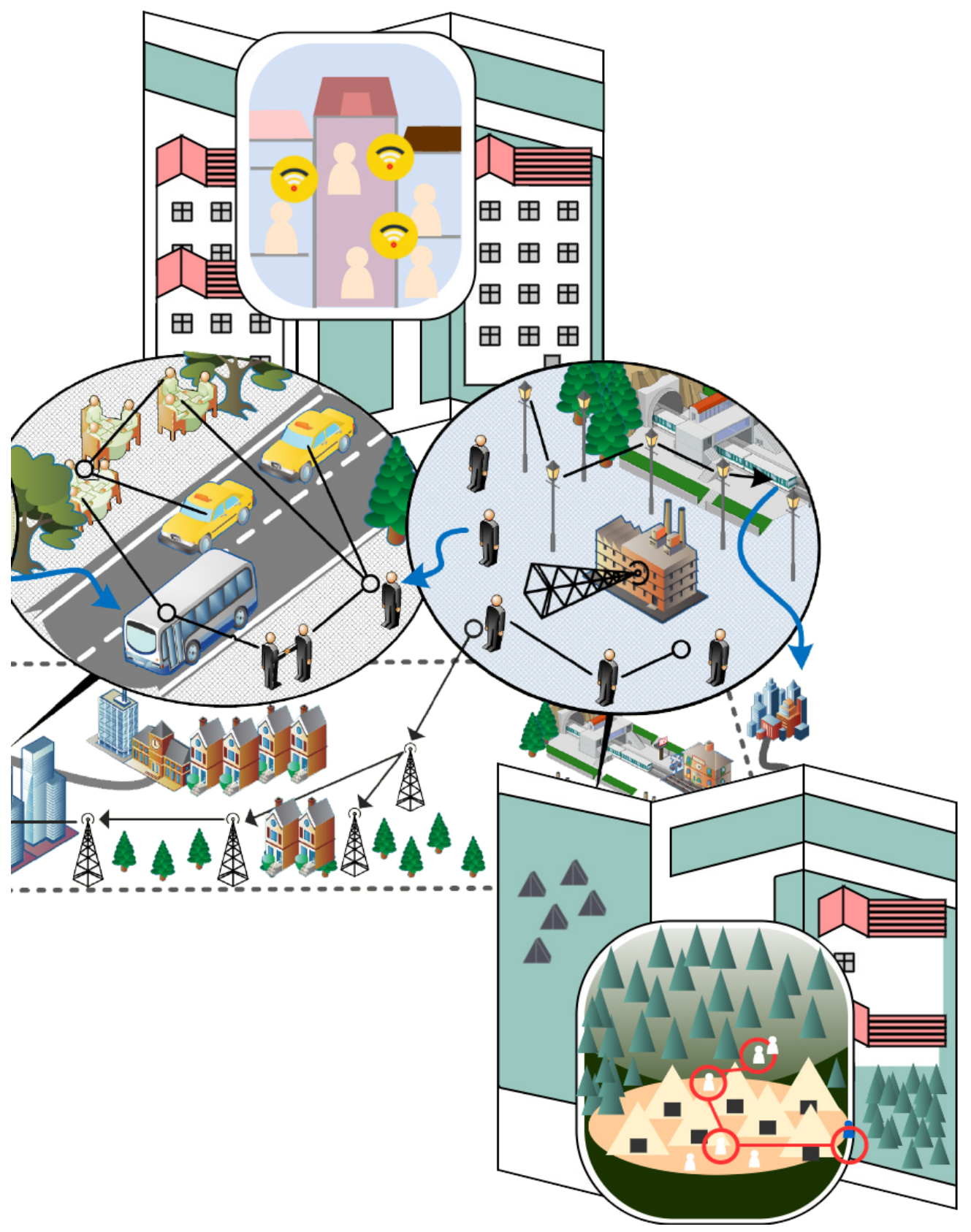


- Tactical Environments: There might be specialized groups or experts who might require opportunistic communications to fulfill a privatized tactical networking scenario. For instance, mobile bill collection operators can opportunistically send or gather wireless information to or from residential areas, respectively. As another example, drivers or motormans operating mass transit units such as buses, trams, subways circulating in a city can disseminate various information at certain intervals based on a scheduled planning.

- Social Environments: In social space, people gather at several locations of interest such as business centers, schools, shopping malls and houses, and form several transient groups for particular period of time. This can be exploited to carry various kinds of messages towards different locations not only for opportunistic routing between endpoints, but also for opportunistic dissemination purposes.

Another potential target of OppNets might be consumer-driven businesses such as opportunistic proximity marketing. Producer-generated or vendor-marketed information can be spread to public through mobility of people. For instance, shop owners can opportunistically disseminate special offers as simple texts messages to their customers anytime and anywhere they want.

- Personalized Environments: Private groups of people such as circle of friends, families, or target groups might necessitate to perform opportunistic group communications for several purposes. For example, detection of an event in a private area can be shared cooperatively with a smartphone-based OppNet. Gathered data can be further analyzed within this network to verify the validity of the event. As another type of example, an OppNet application can be deployed in a hospital to monitor the psychological behaviours or physical activities of patients through sensing with their smartphones and further to inform medicals doctors by means of opportunistic networking.

The list of applications can certainly be extended since the number of existing and visionary OppNet applications are practically endless with the growing enthusiasm within both the research and industrial communities. As OppNet applications become more and more prevalent, there is also a growing interest in the domains of social sciences and studies.

Based on such application areas, users participating in OppNets can cover wide range of areas, where their mobile devices can detect and report information of interest or urgency. OppNet applications may operate by themselves or may be connected to the larger Internet; with further possible scenarios and functionalities they will certainly make opportunistic computing a key player in the next-generation Internet.

It should be noted that one key characteristic of OppNet applications is that data generated by them may be irregular in terms of format, size, and frequency. It should be also noted that a multitude of applications can run at the same time on a same network. This may generate information richness as well as may result in information overload. Users or their devices can create, receive, and share basic kinds of different content over different applications. Overall, all information generated in an OppNet must be interpreted correctly and efficiently. 


\subsubsection{System Characteristics}

While OppNets provide promising additions to mobile ad hoc communications, they introduce unique characteristics presented as follows:

- Mobility: For OppNets, human mobility is a key enabler and a key challenge at the same time. Traditional wireless ad hoc networks consider mobility as an opponent to good functioning. In OppNets, however, mobility is considered as the fundamental facilitator for information routing or dissemination. Despite its advantages, mobility poses the following inter-related complexities:

- Intermittent connectivity: The highly-dynamic essence of OppNets leads to a prevailing intermittent connectivity between devices. Due to stochastic mobility, inter-contact times of devices vary a lot, and are unpredictable in most cases.

- Density variation: Apart from mobility, OppNets builds on density of devices to improve operability. Mobility creates a state of flux in device orientations and therefore network density varies from being very sparse to very dense.

- High latency: Sending information from one point to another can take quite some time due to intermittent connectivity and varying network density.

- Inconsistent data rates: Due to fluctuating wireless conditions, bandwidth is unreliable most of the time.

- Availability: Since OppNet protocols are based on replication and delay-tolerance at the same time, participating devices form a built-in redundancy in terms of information availability. Thus, OppNets have the potential to continue their operation even when arbitrary failures occur.

- Expandability: OppNets are able to grow or shrink incrementally with new users.

- Device heterogeneity: Comprising of many different device types, models, and operating system platforms leads to the following issues to be addressed:

- Platform inconsistencies: Devices have different hardware and software that might obstruct direct communication with others. Even for the same type or model of devices, for example, their wireless adapters might show differences, or, their operating systems might allow/disallow different networking services because of different versions.

- Protocol mismatches: Not all interfaces of the widely-accepted WLAN and WPAN protocols (Wi-Fi and Bluetooth) are same. Offered for mobile devices, some can be only used with only infrastructure-based support, limited number of them have ad hoc support, some has not P2P support at all. On the other hand, operating systems restrict the functionalities or features of these protocols.

- Information irregularity: Data generated by different systems may be irregular in terms of format, size, and frequency. For instance, different models of a same type of sensor may generate an output with different time intervals and with different precision/accuracy values. 


\subsubsection{SySTEM REQUiREMENTS}

For the development of OppNets, general system requirements that remain a continuous concern are described in the following:

- Adaptability: Providing a self-organized OppNet operability under ever-changing network dynamics is the biggest development challenge. Adaptability requirements are collected under the following sub-headings:

- Self-configuration: System should adapt automatically to dynamically changing topologies. Enabling connectivity between devices of heterogeneous networks is another concern. On the other hand, as the device heterogeneity is a concern for direct communications, impromptu interoperability must be taken into account.

- Self-healing: System should be tolerant to the high number of disruptions in case of short communication windows between devices. On the other hand, contentions due to high device density have to be overcome.

- Self-optimization: System should monitor and tune resources automatically. Self-optimization should enable system to efficiently utilize network resources based on shared context in an OppNet.

- Rapid information delivery: In view of frequent disconnections, the data transmissions must be completed in short period of times.

- Scalability: In view of frequent disconnections and density variations, efficient and effective distribution of information toward intended destination(s) require unique scalability solutions based on the networking application.

- Universal Availability: OppNets applications intended for public space need generic networking solutions in order to increase people's participation.

- Multiple Application Support: A scheme that handles different content generated by different applications is necessary.

- Simplicity: The following must be achieved to make a system lightweight:

- Short Messages: An OppNet is not necessarily the most efficient solution for delivering high bandwidth data streams, therefore has to be designed to be efficient for the delivery of small, irregular, and versatile data packets.

- Energy efficiency: Contrary to traditional wireless ad hoc networks, energy is not a primary concern of OppNets. Mobile devices used by people can be recharged continually. Moreover, consumer electronics used as stationary wireless units are plugged to the electric line. Although energy is not a primary concern, energy efficiency of OppNet systems is still important for maximized lifetime. 


\subsection{Thesis Scope}

In view of the above-presented characteristics and requirements, the overall focus of this thesis is to provide lightweight integration of any network opportunity that can occur between mobile users anytime and anywhere in daily life. This integration covers the efficient distribution of various kinds of information shared through several types of OppNet applications.

This thesis focuses on the practical solutions for opportunistic ad hoc networking scenarios as well as for efficient coordination of related OppNet applications running over their corresponding networks. Accordingly, the scope of the thesis concentrates solely on deriving a universal ad hoc networking architecture intended for community-oriented OppNet applications. This section points out our motivation and objectives of this thesis regarding the research question of this thesis. Furthermore, this section describes our approach to the research problem.

\subsubsection{Research Motivation}

The broad range of emerging OppNet applications requires the challenges briefly presented in Section 1.2 to be overcome in an efficient and effective manner. Apart from these challenges, the general requirements of OppNets such as adaptability and scalability remain as important concerns. The utilization of smart mobile devices for OppNets also introduce universal availability problems to be addressed. Overall, these limitations are a disincentive to the development and operation of OppNet applications for personal and common use in daily life.

Despite their advancing features, today's smart mobile devices such as smartphones, tablets, and laptops are onerous to automatically establish mobile ad hoc connections. The challenges increase when the highly-dynamic nature in opportunistic communications is taken into account. Almost all of the smart mobile devices accommodate the commonly-accepted Wi-Fi and Bluetooth interfaces such as Wi-Fi Ad Hoc, Wi-Fi Direct, Wi-Fi Hotspot, and Bluetooth Low Energy. Thoroughly investigated in Chapter 2, these interfaces bring along significant limitations for efficient and reliable mobile networking:

- They lack of self-organization. Prior to a connection, Classic Bluetooth and Wi-Fi Direct enforce secure pairing to unfamiliar devices [29]. Connections that are formed over Wi-Fi Ad Hoc mode require a data forwarding protocol that maintains dynamic topologies [30]. Connecting multiple clients via Wi-Fi Hotspot devices also requires a selforganizing data routing protocol [31].

- Mobility causes frequent disconnections/reconnections that increase routing overhead. Devices consume scarce bandwidth and energy for route rearrangements [32]. Besides, high density causes bandwidth-related issues [33]. These challenges introduce scalability problems for OppNets.

- The widely-used mobile operating systems such as Android, iOS, Windows Phone, and BlackBerry BBOS restrict several functions of these interfaces. 
For instance, Wi-Fi Ad Hoc mode can be activated only under root access privileges on Android and is natively unsupported by iOS, Windows Phone, and BlackBerry OS. On the other hand, Wi-Fi Direct is not supported on $i O S$ by default. Moreover, Wi-Fi Hotspot has rather restricted bandwidth, hence is allowed with limited number of connections, e.g. at most 10 clients on Android; 5 clients on $i O S$ and Windows Phone; and at most. For Classic Bluetooth piconets, this maximum is 7 by default. Overall, these limitations pose both availability and scalability issues.

\subsubsection{Research Овjectives}

Given the motivation, the main focus of this thesis is to build up a universally-viable and practical OppNet architecture that fulfill the requirements of community-oriented ad hoc networking applications running on smart mobile devices. This thesis focuses on the following research question:

How can opportunistic ad hoc communications be achieved with smart mobile devices in a practical, efficient, and generic manner while fulfilling specific requirements of different applications developed for specialized environments?

In view of the distinct requirements of different OppNet types and applications, this thesis expands on our research question by exploring the following sub-questions:

RQ.1 How can a generic OppNet architecture can be designed op top of the wireless access interfaces fitted in smart mobile device platforms?

RQ.2 How can network heterogeneity, in terms of different device and wireless adapter types, be overcome to facilitate the participation of people?

RQ.3 How efficiently can data be routed or disseminated under the conditions of high mobility and high density variations while providing adaptability and energy efficiency?

RQ.4 How can multiple OppNet applications sharing the resources of an OppNet can be managed in a fair manner?

\subsubsection{Research Hypotheses}

Our starting point to answer all of the presented research questions is to propose the general hypothesis that utilizing wireless network identifiers can be beneficial in order to overcome OppNet challenges. More specifically, the following hypotheses are proposed:

- Employing a delay-tolerant protocol which exploits wireless network identifiers can form generality as well as can provide simplicity for networking (RQ.1 and RQ.2).

- Designing wireless network identifiers in accordance with current OppNet application requirements can provide efficiency in data sharing as well as can sustain fairness between devices (RQ.3 and RQ.4). 


\subsubsection{Research Approach}

This thesis approaches the presented research questions by developing a workaround that is applicable on top of both the Wi-Fi and Bluetooth protocol stacks. Therefore, no modification is required in the IEEE 802.11 and IEEE 802.15.1 standards to enable connectivity between devices of Wi-Fi networks or devices of Bluetooth networks, respectively. The workaround assists the progress of lightweight and versatile smart mobile applications that can perform opportunistic short message dissemination and end-to-end routing. It exploits the wireless network identifiers as the basic tool for device discovery and sharing of metadata related to either routing or dissemination of application-specific data.

More specifically, the wireless network identifier fields defined in media access control (MAC) layer are encoded into and further advertised as metadata information. This metadata is denominated as opportunistic beacon. Opportunistic beacons are capable of being modified on any kind of MAC presented in smart mobile devices. As a result, devices utilizing same $\mathrm{PHY} / \mathrm{MAC}$ can discover metadata regardless of differences reflected by their operating system platforms or wireless adapters.

The workaround can achieve lightweight opportunistic communications in two different approaches presented as further layers on top of the network layer:

- The first approach is a connection-free method specifically intended for disseminationbased scenarios. This method exploits opportunistic beacons as message carriers.

- The second approach, on the other hand, is a connection-based method specifically intended for routing-based scenarios. In this method, opportunistic beacons are utilized in order to efficiently manage routing in a distributed way.

Figure 1.5 shows the position of the tasks and mechanisms on the protocol stack. An opportunistic beacon, used in either the first approach or second approach, can network multiple of devices without requiring connection protocols. Our main concentration in this thesis is to investigate the networking potential of the first approach in different setups and scenarios.

FIGURE 1.5

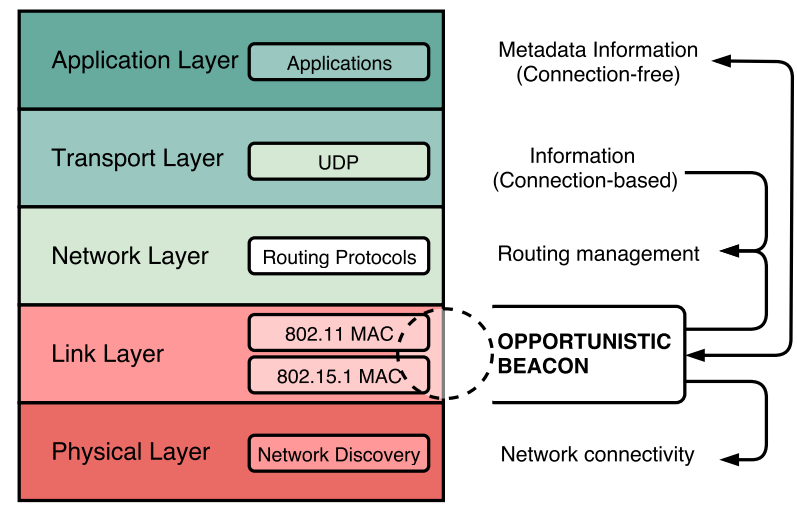

The approach presented on the generalized protocol stack 


\subsection{Thesis Contributions}

With regard to the presented research question and approach, the contributions presented in this thesis are as follows:

\section{Contribution 1: Design and analysis of a community-oriented and context-aware opportunistic networking architecture for smart mobile devices}

A universal and energy-efficient OppNet architecture that can work on top of either Wi-Fi or Bluetooth protocol stacks is devised for smart mobile platforms. Within the architecture, a new set of quality-of-service (QoS) requirements are defined for multiple application support during opportunistic routing. In addition, as previously mentioned in Section 1.3.3, the concept of opportunistic beacons is offered to enable and facilitate networking between devices. Opportunistic beacons enable lightweight and instantaneous data forwarding in ad hoc fashion and regulates data routing. This work is submitted for publication as in the following paper:

- Cocoon: A Lightweight Opportunistic Networking Middleware for Community-oriented Smart Mobile Applications, with H. Scholten and P. J. M. Havinga, Computer Networks, Special Issue on Cyber-Physical Systems for Mobile Opportunistic Networking, under final revision, to be published in September 2016.

\section{Contribution 2: Design and analysis of a lightweight and scalable opportunistic ad hoc data dissemination protocol for smart mobile devices}

A lightweight opportunistic ad hoc data dissemination model is developed which can be employed on any kind of smart mobile device platform. The model is developed in company with a highly-scalable but low-throughput data switching protocol using opportunistic beacons. The protocol is comprehensively tested with different time parameters regarding the wireless operations used in the protocol. These time parameters are further used in the implementation of the model as a simulation model which is validated with real-world experiments. With extensive simulations, several enhancements are offered for the model. This work appeared in the following paper:

- Opportunistic Beacon Networks: Information Dissemination via Wireless Network Identifiers, with H. Scholten and P. J. M. Havinga, in Proceedings of the 5th IEEE International Pervasive Computing and Communications (PerCom) Workshop on the Impact of Human Mobility in Pervasive Systems and Applications (PerMoby'16), Sydney, Australia, March 2016. 


\section{Contribution 3: Evaluation of the presented protocols in different application areas}

The protocol given in Contribution 2 is evaluated within the following use case studies:

Providing communications in highly-dense places. This work appeared in:

- Friend-to-Friend Short Message Service with Opportunistic Wi-Fi Beacons, with H. Scholten and P. J. M. Havinga, in Proceedings of the 7th IEEE International Pervasive Computing and Communications (PerCom) Workshop on Pervasive Collaboration and Social Networking (PerCol'16), Sydney, Australia, March 2016.

The protocol is tested with an enhancement in different network densities varying from very dense to very sparse. The work appeared in:

- BLESSED with Opportunistic Beacons: A Lightweight Data Dissemination Model for Smart Mobile Ad-Hoc Networks, with H. Scholten and P. J. M. Havinga, in Proceedings of the 10th ACM Mobile Computing and Networking (MobiCom) Workshop on Challenged Networks (CHANTS'15), Paris, France, September 2015.

As another specific use case, the protocol is tested in vehicular environments. This work appeared in the following paper:

- An Ad-Hoc Opportunistic Dissemination Protocol for Smartphone-Based Participatory Traffic Monitoring, with F. Seraj, H. Scholten, N. Meratnia, and P. J. M. Havinga, in Proceedings of the 82nd IEEE International Vehicular Technology Conference (VTC'15 Fall), Boston, MA, USA, September 2015.

The following relevant contributions are not directly included in the thesis but are cited throughout the thesis:

- RoRo-LT: Social Routing with Next-Place Prediction from Self-Assessment of Spatiotemporal Routines, with H. Scholten and P. J. M. Havinga, in Proceedings of the 10th IEEE International Conference on Ubiquitous Intelligence and Computing (UIC'13),

Vietri Sul Mare, Italy, December 2013.

- Introspection-based periodicity awareness model for intermittently connected mobile networks, with H. Scholten and P. J. M. Havinga, the 4th International Conference on Mobile, Ubiquitous, and Intelligent Computing (MUSIC'13), September 2013, South Korea.

- Opportunistic Data Dissemination in Mobile Phone Sensor Networks, with H. Scholten and P. J. M. Havinga, Extended Abstract, Adjunct Publication of the ACM International Conference on Ubiquitous Computing (UbiComp'13), Zurich, Switzerland.

- A Smartphone Based Method to Enhance Road Pavement Anomaly Detection by Analyzing the Driver Behavior, with F. Seraj, K. Zhang, N. Meratnia, P. J. M. Havinga, the 4th ACM International Pervasive and Ubiquitous Computing (UbiComp) Workshop on Pervasive Urban Applications (PURBA 2015), September 2015, Osaka, Japan. 


\subsection{Thesis Organization}

The works presented in this thesis can be examined under four main parts:

i) Background, ii) Design \& Evaluation, iii) Applications, and iv) Conclusion.

The contents presented under these parts are summarized in Figure 1.6.

Background is covered in Chapters 1-2.

In this chapter, the basics of opportunistic networking are briefly discussed to clarify our research motivation and objectives.

Chapter 2, in addition to this chapter, emphasises the characteristics and challenges of OppNets in more depth by introducing the limitations of today's smart mobile platforms. Furthermore, Chapter 2 presents the related works regarding the design of OppNets.

Design \& Evaluation is presented in Chapters 3-4.

Chapter 3 which corresponds to Contribution 1 introduces our OppNet architecture, Cocoon (Community-Oriented Context-Aware Opportunistic Networking).

Chapter 4 which corresponds to Contribution 2 describes our connection-free networking model, called Opportunistic Beacon Networking (OBN), and presents the protocols devised on top of OBN.

Applications are presented in Chapter 5.

Chapter 5 gives the implementation and evaluation details of the presented applications which use the presented protocols presented in Chapters 3-4. For all of these applications, the routing method is based on OBN, and therefore their protocols are repeatedly described with additional design features. The evaluation of each presented application corresponds to Contribution 3.

Conclusions are drawn in Chapter 6.

Chapter 6 describes the conclusions and inferences drawn in this thesis and further expresses future prospects and open research directions.

Appendices give the acronyms and notations used throughout the thesis and additionally presents several important points our implementations. In Oppliqué API Guide, the details of the simulation used in the thesis are explained and the related setups set up for the evaluations are given. In Cocoon API guide, the classes, modules, and functionalities of the Cocoon implementation is explained. 


\begin{tabular}{|c|c|c|c|}
\hline Chapter 1 & Chapter 3 & Chapter 5 & Chapter 6 \\
\hline Mobile communications & Cocoon & Overview & Final Overview \\
\hline $\begin{array}{l}\text { Retrospectives and } \\
\text { prospectives }\end{array}$ & $\begin{array}{l}\text { System requirements } \\
\text { System characteristics } \\
\text { System components }\end{array}$ & \multirow[t]{3}{*}{$\begin{array}{l}\text { Application objectives } \\
\text { Networking types }\end{array}$} & \multirow[t]{2}{*}{$\begin{array}{l}\text { Reflections \& Implications } \\
\text { Key findings }\end{array}$} \\
\hline MANETs & \multirow{2}{*}{ System Architecture } & & \\
\hline Characteristics & & & Contributions Revisited \\
\hline Application areas & $\begin{array}{l}\text { Connectivity } \\
\text { Networking } \\
\text { Service Management }\end{array}$ & oppline & \multirow{4}{*}{$\begin{array}{l}\text { Availability } \\
\text { Scalability } \\
\text { Adaptability } \\
\text { Ease of use } \\
\text { Energy efficiency } \\
\text { Validated Tests } \\
\text { Validated Applications }\end{array}$} \\
\hline $\begin{array}{l}\text { Characteristics } \\
\text { Application areas }\end{array}$ & Opportunistic Beacons & \multirow{5}{*}{$\begin{array}{l}\text { Motivation \& Objectives } \\
\text { Communication Model } \\
\text { Protocol Design } \\
\text { Implementation } \\
\text { Networking Tests } \\
\text { Performance Analysis } \\
\text { Conclusions }\end{array}$} & \\
\hline OppNets & $\begin{array}{l}\text { Properties } \\
\text { Pnces }\end{array}$ & & \\
\hline $\begin{array}{l}\text { Characteristics } \\
\text { Application areas }\end{array}$ & Applicability Study & & \\
\hline \multirow[t]{2}{*}{ Chapter 2} & QoS & & Future Research Directions \\
\hline & $\begin{array}{l}\text { Service Characterization } \\
\text { Application Handling }\end{array}$ & & \multirow{3}{*}{$\begin{array}{l}\text { Broadening the context } \\
\text { The future of beacons } \\
\text { Complex scenarios } \\
\text { Security considerations }\end{array}$} \\
\hline State-of-the-Art & Chapter 4 & \multirow{2}{*}{ B L ES S E D } & \\
\hline \multirow{2}{*}{$\begin{array}{l}\text { Available Technologies } \\
\text { Mobile O/S Support } \\
\text { Challenges in OppNets }\end{array}$} & & & \\
\hline & OBN & \multirow{5}{*}{$\begin{array}{l}\text { Motivation \& Objectives } \\
\text { Communication Model } \\
\text { Protocol Design } \\
\text { Implementation } \\
\text { Networking Tests } \\
\text { Performance Analysis } \\
\text { Conclusions }\end{array}$} & Appendices \\
\hline Design Considerations & $\begin{array}{l}\text { Network Model } \\
\text { Protocol } \\
\text { Implementations }\end{array}$ & & \multirow[b]{2}{*}{ Acronyms \& Notations } \\
\hline \multirow{2}{*}{$\begin{array}{l}\text { Principles, requirements, } \\
\text { related work }\end{array}$} & Experiments & & \\
\hline & Validation & & \\
\hline Systems & $\begin{array}{c}\text { Parameter Testing } \\
\text { Networks }\end{array}$ & & \\
\hline \multirow{2}{*}{$\begin{array}{c}\text { Network Architectures } \\
\text { Connectivity Schemes } \\
\text { Applications \& Services } \\
\text { Beacon Profiles }\end{array}$} & Enhancements & ID VADISS & Oppliqué · API Guide \\
\hline & $\begin{array}{l}\text { Time-adaptiveness } \\
\text { Message-adaptiveness } \\
\text { Dual radio utilization }\end{array}$ & \multirow{3}{*}{$\begin{array}{l}\text { Motivation \& Objectives } \\
\text { Communication Model } \\
\text { Protocol Design } \\
\text { Implementation } \\
\text { Networking Tests } \\
\text { Performance Analysis } \\
\text { Conclusions }\end{array}$} & \\
\hline Forwarding Protocols & Performance Analysis & & Cocoon · API Guide \\
\hline $\begin{array}{l}\text { Replication techniques } \\
\text { Context-awareness }\end{array}$ & $\begin{array}{c}\text { Scalability } \\
\text { Parameter Effects } \\
\text { Comparisons }\end{array}$ & & 8 \\
\hline Background & $\begin{array}{l}\text { Design \& } \\
\text { Evaluation }\end{array}$ & Applications & Conclusions \\
\hline
\end{tabular}

Thesis Organization 



\section{State-of-The-Art}

THIS CHAPTER PRESENTS A HOLISTIC PICTURE OF THE STATE-OF-THE-ART BY EXAMINING THE AVAILABLE TECHNOLOGIES IN SMART MOBILE PLATFORMS AS WELL AS PRESENTING THE RELATED WORKS. INITIALly, the OpPNet apPlications AND PROTOCOLS ARE BRIEFLY DISCUSSED IN TERMS OF THE PRINCIPLES AND REQUIREMENTS IN THEIR DESIGN AND DEVELOPMENT. SECONDLY, THE LIMITATIONS, CHALLENGES, AND OPPORTUNITIES OF THE COTS SHORT-RANGE WIRELESS NETWORKING INTERFACES AVAILABLE IN THE POPULAR SMART MOBILE O/S PLATFORMS ARE DISCUSSED. THEIR AVAILABILITY AND APPROPRIATENESS ARE INVESTIGATED FOR THE DEVELOPMENT OF OPPNET SYSTEMS AND ROUTING STRATEGIES. RELATED WORKS ARE GIVEN IN TWO SEPARATE SECTIONS: $i$ ) SYSTEMS AND ii) FORWARDING PROTOCOLS. THE EXISTING SYSTEMS INCLUDE NETWORK ARCHITECTURES, CONNECTIVITY SCHEMES, AND SERVICES EACH REPRESENTING DIFFERENT FOCUS OF INTEREST. INCLUDING OUR ARCHITECTURE, COCOON PRESENTED IN CHAPTER 3, THE PROPOSED SYSTEMS ARE DESCRIBED IN A COMPARATIVE MANNER BASED ON THEIR NETWORKING SUPPORT AND EFFECTIVENESS. FURTHERMORE, THE SMART MOBILE APPLICATIONS THAT ARE COMMERCIALLY-AVAILABLE AND DEVELOPED BY ACADEMIA ARE PRESENTED. ON THE OTHER HAND, FORWARDING PROTOCOLS THAT ARE OFFERED FOR THE UPPER LAYERS OF THE PROTOCOL STACK ARE EXAMINED WITH REGARD TO THEIR CONTEXT UTILIZATION. THE PROPOSED FORWARDING STRATEGIES ARE COMPARED BASED ON A NOVEL CATEGORIZATION. AT THE END OF THE CHAPTER, A SHORT DISCUSSION ON THE STATE-OF-THE-ART IS GIVEN. 


\subsection{OVERVIEW}

OppNets for real-world use are gradually emerging. Aiming at smart mobile device platforms, the number of proposed systems is increasing. OppNets have the potential to revolutionize the way the humanity performs communications by addressing the limitations of situated wireless counterpart as well as by introducing new applications to the field of mobile communications. Nevertheless, the state-of-the-art represents several challenges and limitations that hinder efforts to tackle the intrinsic characteristics of opportunistic communications. Before going into the details of the state-of-the-art, this section presents a brief overview about the principles and requirements of the OppNet applications and protocols.

\subsubsection{OppNet Applications}

OppNet applications can be used in a great number of mobility-assisted scenarios. The first and the most important principle of mobility-assisted applications is to cope with the connectivity issues among devices. Frequent disconnections and re-connections have to be addressed with a rapid and efficient connectivity scheme. While having promising potentials, the current wireless standards (e.g. Wi-Fi, Bluetooth) have limited support for mobility. Consequently, this lack of support has stimulated little research efforts so far to devise an effective solution for the realization of OppNets.

The majority of the existing systems include small-scale examples, P2P solutions, and delay-tolerant examples which cannot achieve high scalability. In addition, they do not provide lightweight OppNet services, i.e. they focus on performing high-bandwidth data transmissions under stable connectivities rather than supporting information sharing in small sizes and under high mobility. Section 2.3 presents a detailed survey of these systems.

\subsubsection{OppNet Protocols}

In the realm of OppNets, the most widely explored topic is forwarding. The majority of the proposed forwarding protocols relies on theoretical studies. The practical side is often missed out due to the lack of OppNet services in reality.

A forwarding protocol is necessary to optimize OppNet resources. The unpredictable mobility of devices makes forwarding a difficult task. The naive approaches offered in the research domain often result in poor performance when network resources get scarce. On the other hand, there are forwarding strategies that link mobility together with context awareness. The context-aware forwarding approaches mostly focus on the user properties such as social properties to better exploit mobility in resource optimization. Section 2.4 presents a detailed survey of the proposed forwarding strategies. 


\subsubsection{Chapter Organization}

The rest of chapter is organized as follows: Section 2.2 explains the available wireless ad hoc networking technologies presented for smart mobile O/S platforms. Section 2.3 presents the existing works that put OppNets into practice. These works include systems that comprise either delay-tolerant connectivity schemes, or network architectures, or services, or integral solutions that comprise all. Section 2.4 presents the existing forwarding protocols based on their replication strategies and their context utilization. Section 2.5 gives the concluding remarks with a brief discussion on the state-of-the-art.

The existing approaches to realize OppNets mainly concentrate on the topics of either networking implementations, or opportunistic routing, or only the implementation of peoplecentric opportunistic communication applications. There also exist integral proposals which are presented as network architectures or connectivity schemes. To clearly show their focus of interest, Figure 2.1 presents the existing works based on a separation on the generalized version of the protocol stack.

The network architectures and connectivity schemes focus mainly on the the network layer protocols supported with store-carry-forward routing, i.e. the ones implemented with the consideration of delay/disruption tolerance. Additionally, some of them present several adaptations on the PHY/MAC layer. Besides, OppNet services are mainly studied in the application layer. Some of them also suggest new connectivity schemes presented on top of the network layer.

The forwarding protocols, on the other hand, are the studies presented in a mid-layer between the transport and application layers. The protocols operate with a delay-tolerant fashion which is different than the traditional routing approaches presented for the transport layer. The protocols also utilize a level of context awareness provided by the application layer. The context utilized is often user context, but can be also related to the network itself or about the networking environment.
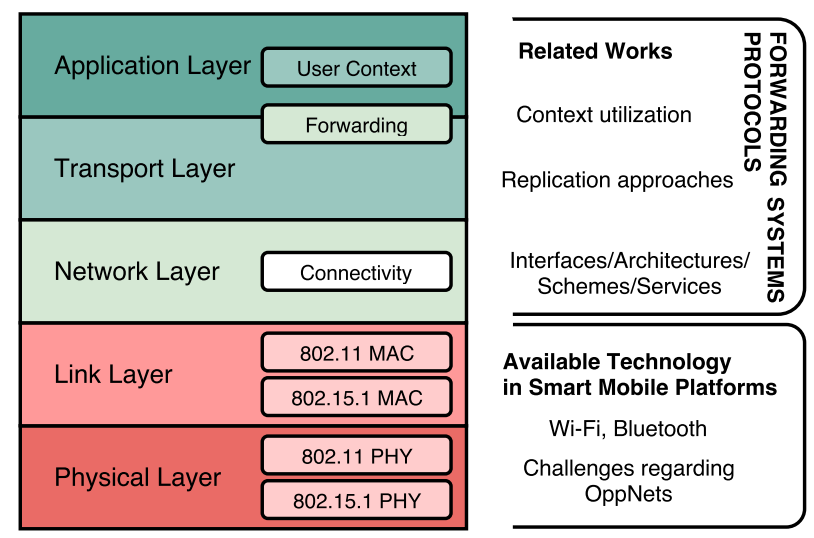

Chapter Organization: State-of-the-art presented on the generalized protocol stack 


\subsection{Ad Hoc Networking Technologies in Smart Mobile Platforms}

To actualize people-centric OppNet architectures and applications, Wi-Fi and Bluetooth are the only predominant WLAN and WPAN standards available on the market, respectively. Worldwide, almost all mobile communication devices support Wi-Fi access [34]. Likewise, Bluetooth is the long-running short-range access standard for portable devices, including smart mobile devices and majority of the consumer electronics [35]. This section elaborates on these standards regarding their ad hoc networking properties and capabilities.

Over the last decade, the working groups of IEEE 802.11 and IEEE 802.15.1 have introduced several Wi-Fi and Bluetooth connectivity standards, respectively, based on differentiated amendments.

For Wi-Fi, the so-far available interfaces on mobile O/S platforms are as follows:

Wi-Fi Ad Hoc, which is dedicated for communications in a decentralized form [30], i.e. without requiring a centralized AP. Instead, each device on a network built on Wi-Fi Ad Hoc mode serves as a separate AP. By default, IEEE 802.11 MAC introduces its Basic Service Set (BSS) to enable access to infrastructure-based Wi-Fi networks [36]. In Wi-Fi Ad Hoc mode, on the other hand, Independent BSS (IBSS) is used to provide direct access between network devices. IBSS networks are freestanding, thus they cannot connect to any other BSS networks. Any supporting device can start an IBSS, and there exists no hierarchy between IBSS devices. A device willing to join an IBSS with a certain wireless network identifier will scan to see if the network already exists, by passively listening on the channel and receiving beacons from other IBSS devices or by sending probe requests. If an existing IBSS is detected its identifier is taken over and data frames can be exchanged directly between all IBSS nodes within radio reach.

Wi-Fi Direct, which is an interface that is not introduced as an IEEE standard, but is defined under Wi-Fi Alliance Technical P2P Specification [37]. Wi-Fi Direct devices can connect to each other and form ad hoc groups, usually one-to-one, but also oneto-many. A default Wi-Fi Direct connection is based on a negotiation between Wi-Fienabled devices to decide on the networking roles: One of them is assigned for the traditional role of AP called group owner while others stay in client mode. Each device uses its global MAC address as Device ID during discovery and negotiation, and a temporary local MAC address for all frames within a Wi-Fi Direct group.

Wi-Fi Hotspot, which is the interface that allows those devices to function as mobile APs. Majority of the modern Wi-Fi-enabled devices are capable of functioning in either AP or infrastructure mode. From this aspect, it is also possible to build up ad hoc connections with AP-client orientations in Wi-Fi networks. 
For Bluetooth, the so-far introduced interfaces on mobile O/S platforms are as follows:

Bluetooth Classic, which is initiated and being developed by the Bluetooth Special Interest Group. Bluetooth Classic is a wireless technology for exchanging data over short distances. The basic unit of ad hoc networking in Bluetooth Classic is a piconet, comprising a master and from 1 to 7 active slave devices. A device in a piconet can also exist as part of another piconet and may function as either a slave or master in each piconet. This form of overlapping is called a scatternet.

Bluetooth Low Energy (BLE), or Bluetooth Smart, or Bluetooth 4.0+, which is introduced in 2010 by the Bluetooth Special Interest Group as a new low-power, lightweight standard [38]. Its availability is increasing in mobile devices [39]. With BLE-enabled devices, forming ad hoc networks also becomes easier in comparison to Bluetooth Classic. BLE employs a generic data protocol called the Attribute Protocol (GATT), which uses attribute IDs to store services, characteristics, and data in terms of BLE beacons. Utilizing BLE beacons, devices can form connections over the GATT protocol. By definition, GATT and connections are exclusively handled, i.e. connections are administered in a different layer. For this connectivity layer, two BLE modes are defined: peripheral and central which are similar to master and slave modes defined in Bluetooth Classic.

\subsubsection{General Characteristics}

Table 2.1 outlines the general properties of the above-presented interfaces. The theoretical wireless radio range is limited to from $10 \mathrm{~m}$ to $20 \mathrm{~m}$ in Classic Bluetooth adapters whereas $\mathrm{Wi}-\mathrm{Fi}$ and BLE adapters can support ranges up to $90 \mathrm{~m}$. These ranges are highly suitable for OppNets. However, all of these interfaces are offered to serve as stable ad hoc (or P2P) routing protocols between mobile devices, therefore are out of keeping with the general OppNet requirements such as mobility and density variations. Furthermore, they employ costly and tardy connectivity protocols which do not suit with the necessity of lightweight OppNet application services.

TABLE 2.1: PHY/MAC Interface Properties

\begin{tabular}{|c|c|c|c|c|c|}
\hline $\begin{array}{l}\text { PHY/MAC } \\
\text { Interface }\end{array}$ & $\underset{\text { Limit }}{\text { Group }}$ & $\begin{array}{r}\text { Max. Data } \\
\text { Rate }\end{array}$ & $\begin{array}{l}\text { Radio } \\
\text { Range }\end{array}$ & $\begin{array}{r}\text { Editable ID } \\
\text { Length }\end{array}$ & $\begin{array}{r}\text { Authentication/ } \\
\text { Pairing }\end{array}$ \\
\hline Wi-Fi Ad-Hoc & $\mathrm{N} / \mathrm{A} \star$ & $11 \mathrm{MBps}$ & $\lesssim 90 \mathrm{~m}$ & 32 B ASCII & Automatic connection \\
\hline Wi-Fi Direct & $2-10 \bullet$ & $250 \mathrm{MBps}$ & $\lesssim 90 \mathrm{~m}$ & 32 B ASCII & Manual authentication \\
\hline Wi-Fi Hotspot & $2-11 \bullet$ & $54 \mathrm{MBps}$ & $\lesssim 90 \mathrm{~m}$ & 32 B ASCII & Automatic connection \\
\hline Bluetooth 1.0 & 8 & $723 \mathrm{KBps}$ & $\lesssim 10 \mathrm{~m}$ & 248 B UTF-8 & Initial manual pairing \\
\hline Bluetooth 2.1 & 8 & $3 \mathrm{MBps}$ & $\lesssim 20 \mathrm{~m}$ & 248 B UTF-8 & Initial manual pairing \\
\hline Bluetooth 3.0 & 8 & $24 \mathrm{MBps}$ & $\lesssim 20 \mathrm{~m}$ & 248 B UTF-8 & Initial manual pairing \\
\hline BLE & $\mathrm{N} / \mathrm{A} \star$ & $260 \mathrm{KBps}$ & $\lesssim 90 \mathrm{~m}$ & 128 bits & Automatic connection \\
\hline \multicolumn{6}{|c|}{$\star$ No theoretical limit is defined. } \\
\hline
\end{tabular}


Wi-Fi Ad Hoc supports high bandwidth and theoretically sets no limit for the networking peers. Due to their restricted bandwidths, Wi-Fi Direct and Wi-Fi Hotspot are allowed with a limited number of connections. A Bluetooth piconet can support 8 devices (1 master and 7 active slaves) at most. On the contrary, BLE has no theoretical limit for the number of peripheral device connections.

In terms of establishing a hand-shake between peer devices, interfaces reflect differences. For Wi-Fi Ad Hoc, Wi-Fi Hotspot, and BLE, connections are established without user intervention. For Wi-Fi Direct, on the other hand, manual authentication is required for every connection. For Bluetooth Classic, manual pairing is required between the devices establishing connections for the first time.

For network discovery, wireless interfaces utilize a specific field called wireless network identifier field, or simply network name. For wireless network identifier, Wi-Fi uses Service Set Identifier (SSID) field. By default, SSID field is defined in the standard MAC beacon frames as at most 32 ASCII bytes in length. Bluetooth Classic uses a field called Bluetooth Network Name which can be at most 248 UTF- 8 bytes in length. BLE uses Universally Unique Identifier (UUID) as wireless network identifier which is defined as 128 bits in length.

\subsubsection{Challenges regarding OppNet Characteristics}

In relation to the intrinsic OppNet characteristics such as mobility and density variations, the presented interfaces response in a way which requires additional considerations in both the network design and network operation. These considerations are collected under the following headings:

\section{Neighbor Discovery}

Neighbor discovery is one of the key mechanisms of PHY/MAC to efficiently administer selforganizable mobile systems like OppNets [40]. The purpose of service discovery is to get knowledge about existing network services and their contact information as well as to secure access to the services.

In Wi-Fi Ad Hoc mode, neighbor discovery is a straightforward but energy-hungry process [41]. The process is continuous, i.e. the shared channel is passively listened all the time to detect and match IBSS probe or request beacons.

In Wi-Fi Infrastructure mode, the Wi-Fi channels are scanned for the advertised BSS.

In Wi-Fi Direct, on the contrary, neighbor discovery phase turns into a complex task. The discovery process usually starts by performing a traditional Wi-Fi scan (active or passive). Just after this scan, an interface-specific discovery algorithm is executed. First, a Wi-Fi channel is selected for scanning. For the selected channel, the algorithm alternates between two states: a search state to perform active scanning for each channel by sending probe requests, and a listen state to perform scanning in the selected channel. As a result of this comparably long process, during short communication windows between devices, Wi-Fi Direct might not complete the device discovery process in time. 
Applying to all Wi-Fi interfaces, IEEE 802.11 scan process is time-varying based on the scan type, channel conditions, and the number of channels scanned.

In Bluetooth Classic, the neighbor discovery phase may take prohibitively long time [42]. As a consequence, this may significantly impact networking in case of frequent disconnections due to mobility. Another important factor that affects the neighbor discovery time is the availability of a shared channel. Since Bluetooth is based on Code Division Multiple Access (CDMA) baseband processing, communication between any pair of devices is impossible until their clocks are synchronized. Especially when the number of devices increases, this type of baseband design facilitates scalability, but makes the discovery process longer. Furthermore, Bluetooth discovery protocol is costly in terms of power [32] and necessitates initial manual pairing between peers.

In BLE, on the other hand, GATT offers low-power and simple device discovery with two more flexible options for querying service or characteristics attributes defined in UUID: i) discovery of all primary services, and ii) discovery of a primary service by querying a specific service UUID. Thanks to its simplicity, BLE neighbor discovery is fast and robust even under high device mobility and density.

\section{Network Management}

For the presented interfaces, their flexibility in terms of dynamic group formation is closely related with the adaptability requirement of OppNets. Both networking and routing must be robust against device joins and disjoins.

Wi-Fi Ad Hoc mode, as a dedicated interface for ad hoc networking, shares IBSS to participating devices within a network. Since there exist no concept like network owner, master, or AP, Wi-Fi Ad Hoc mode is capable of handling dynamic topology changes. Theoretically having no limit for the number of connections, Wi-Fi Ad Hoc enables high network scalability if low interference is assured [30].

As a P2P standard, Wi-Fi Direct does not respond well to dynamic topology changes. The reason is that the negotiation process between the devices is burdensome. In case the group owner disappears, left devices have to start over a new negotiation process. Therefore, Wi-Fi Direct is more suitable for group communications under stable connectivity [31]. On the other hand, under varying network densities, the adaptability of Wi-Fi Direct remains as a big question for OppNet adaptability.

One other approach is to utilize Wi-Fi Hotspot feature to share Wi-Fi medium as AP with connected clients. Similar to Wi-Fi Direct, Wi-Fi Hotspot suffers in case of mobility since connections are coordinated through AP. As a consequence, Wi-Fi Hotspot networks are often subject to low scalability [43].

Bluetooth is a dedicated protocol for single-hop network arrangements. As a consequence, Bluetooth Classic also brings significant limiting factors for wide deployment of OppNets. First and foremost, it necessitates manual pairing between unknown peers, i.e. forming a Bluetooth piconet is possible between only pre-paired devices. This simply means that opportunistic contacts that are encountered for the first time cannot be included into networking without user intervention. It is also attainable to form a Scatternet with the combination of several piconets. However, Scatternet is neither supported nor feature-limited by the majority of 
Bluetooth adapters. Moreover, mobility causes extremely high overhead due to frequent master/slave disconnections and reconnections. Overall, traditional Bluetooth networks mostly fit to link grouped devices under stable connectivity, and are not sufficient enough for selforganizing OppNets [32].

The networking approach in BLE, on the other hand, is similar to Wi-Fi Hotspot networks. The concept of central/peripheral in BLE is introduced for network connectivity. A peripheral can advertise, to let other devices know its presence, but it is only a central that can actually send a connection request to establish a connection. Multiple centrals can connect to only one peripheral. When the peripheral is lost, all connections have to be reestablished over another peripheral available.

In order to summarize the protocol differences over a simple group formation example, Figure 2.2 depicts how each of the presented interfaces react to the topology changes. Among all, Wi-Fi Ad Hoc mode employs the most reactive protocol against mobility. Nevertheless, like others, it cannot overcome the OppNet necessity of rapid reconfiguration between devices.

\section{Connection Establishment}

In terms of networking performance, OppNets can be drastically affected by mobility and high device density. Due to high mobility, authentication, association, and connection orientation may not be completed in short inter-contact durations. Frequent disconnections/reconnections make Wi-Fi and Bluetooth consume scarce bandwidth and energy [32, 41]. In addition, high device density may cause interference.

\section{Wi-Fi Hotpost, Wi-Fi Direct, BLE}
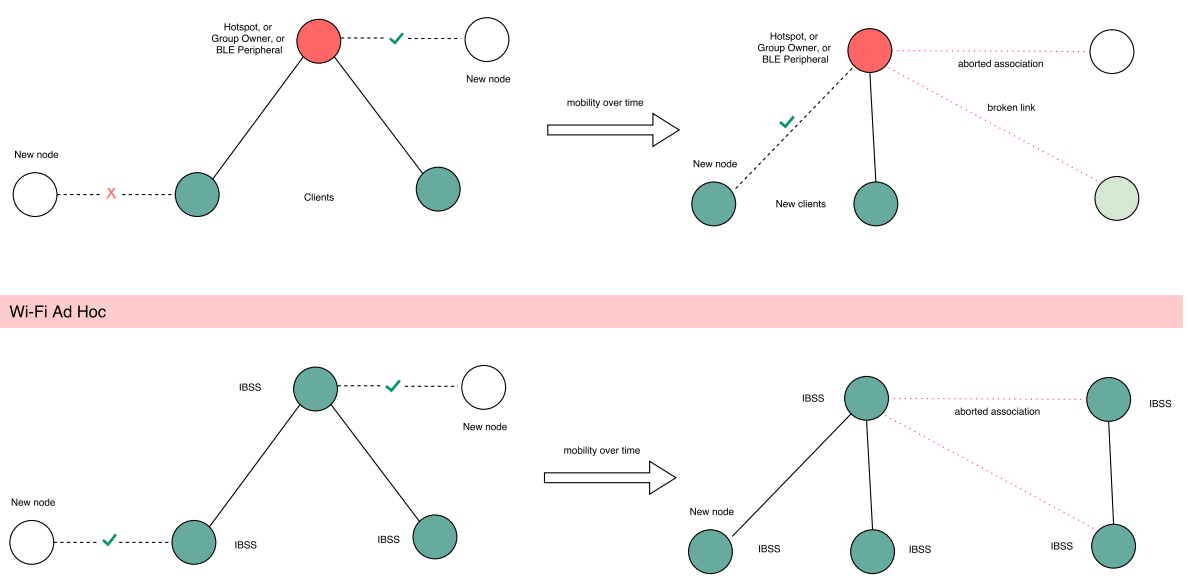

Interface reactions to topology changes 


\subsubsection{Mobile Operating System Support}

The usability of the presented interfaces in mobile O/S platforms reflects differences. Table 2.2 itemizes the interfaces based on their usability on the widely-used mobile O/S platforms. Despite their high adoption, their common usability gets lessened due to divergent $\mathrm{O} / \mathrm{S}$ versions. Furthermore, some functionalities of them are not supported by all mobile O/S platforms. For instance, Wi-Fi Ad Hoc is not supported by default, or is released as a hidden feature, therefore needs strenuous efforts to be used by inexperienced users. Moreover, Wi-Fi Direct is not built in iOS and Windows Phone. Similarly, BLE is restricted in all O/S platforms. Regarding their usability on mobile O/S platforms, Wi-Fi Hotspot and Bluetooth constitute the majority of the commonly-reachable PHY/MAC interfaces.

Another restricting factor is that not all Wi-Fi or Bluetooth adapters are introduced equal on mobile $\mathrm{O} / \mathrm{S}$ platforms. In relation with this fact, the problems that may arise are highlighted in the following:

- From smartphones to tablets to smartwatches to everything in between, devices of different types, different models, and different vendors show high diversity in their affiliated adapters. Table 2.3 gives a list of incompatibility examples for both Wi-Fi and Bluetooth.

- As an example regarding interface mismatches, Wi-Fi Direct necessitates compulsory additions such as the use of IEEE 802.11g, Robust Security Network (RSN), Wi-Fi Protected Access version 2 (WPA2), Wi-Fi Protected Setup (WPS), wireless multimedia extensions for increase QoS. These additions may not be supported by all devices or their platforms.

- As an example for unsupported data rates, Bluetooth Classic has differentiated versions of traffic control defined in its Basic Rate/Enhanced Data Rate (BR/EDR) protocol introduced differently in various Bluetooth adapters types. Different adapter types causes transmissions to be minimized to the lowest data rate.

TABLE 2.2: Mobile O/S Support

\begin{tabular}{lllll}
\hline $\begin{array}{l}\text { PHY/MAC } \\
\text { Interface }\end{array}$ & $\begin{array}{c}\text { Google } \\
\text { Android }\end{array}$ & $\begin{array}{c}\text { Apple } \\
\text { iOS }\end{array}$ & $\begin{array}{c}\text { Windows } \\
\text { Phone }\end{array}$ & $\begin{array}{c}\text { BlackBerry } \\
\text { BBOS }\end{array}$ \\
\hline Wi-Fi Ad-Hoc & $\mathrm{v} 2.2+\star$ & $\mathrm{v} 4.1+\star$ & $\mathrm{N} / \mathrm{A}$ & $\mathrm{N} / \mathrm{A}$ \\
\hline Wi-Fi Direct & $\mathrm{v} 4.0+$ & $\mathrm{v} 6.0+\star$ & $\mathrm{v} 8.1+\star$ & $\mathrm{v} 10.0+$ \\
\hline Wi-Fi Hotspot & $\mathrm{v} 2.3+$ & $\mathrm{v} 4.0+$ & $\mathrm{v} 7.5+$ & $\mathrm{v} 5.0+$ \\
\hline Bluetooth 1.0 & $\mathrm{v} 2.0+$ & $\mathrm{v} 3.0+$ & $\mathrm{v} 7.0+$ & $\mathrm{v} 4.0+$ \\
\hline Bluetooth 2.1 & $\mathrm{v} 2.0+$ & $\mathrm{v} 3.0+$ & $\mathrm{v} 7.0+$ & $\mathrm{v} 4.0+$ \\
\hline Bluetooth 3.0 & $\mathrm{v} 3.2+$ & $\mathrm{v} 3.0+$ & $\mathrm{v} 7.0+$ & $\mathrm{v} 4.0+$ \\
\hline BLE & $\mathrm{v} 4.3+$ & $\mathrm{v} 5.1+$ & $\mathrm{v} 8.1+$ & $\mathrm{v} 10.0+$ \\
\hline
\end{tabular}

$\star$ Requires root permission in Google Android; not supported natively in Apple iOS, Windows Phone, and BBOS.

- BLE Peripheral Mode is supported in Google Android v5.0+; not available in Windows Phone and BBOS. 
- Mobile O/S platforms restrict or withhold several features of these interfaces.

- Wi-Fi Hotspot is allowed with a limited number of connections-at most 10 clients in Google Android and 5 clients in Apple iOS and Windows Phone by default. This number is limited to only a single connection on some Wi-Fi adapters and cannot be changed unless updating adapter firmware.

- Similarly, Wi-Fi Direct is also allowed with a limited number of connections.

- Scatternet is neither supported nor is feature-limited by the some of the Bluetooth adapters. Thus, forming larger networks with multiple piconets becomes impossible.

In light of the available usability, the presented problems have the potential to cast away available contact opportunities, and to hinder the participation of people to the OppNet applications for general public use.

\subsubsection{Discussion}

In terms of network flexibility, Wi-Fi Ad Hoc mode can perform well compared to other interfaces, however, it is costly in terms of power. The same fact holds also for Wi-Fi Direct and Bluetooth Classic. In consideration of common usability, Wi-Fi Ad Hoc mode, Wi-Fi Direct, and Bluetooth Classic have several significant barriers.

Regarding device-to-device negotiations, user intervention is a compelling limitation in OppNet design. User intervention is an enforcement of Wi-Fi Direct and Bluetooth Classic, therefore is a crucial drawback considering opportunistic contacts. Other protocols do not require any manual adjustment or authentication during connection establishment.

Thanks to their ease of use, high adoption, availability, and homogeneity in terms of adapter specifications, Wi-Fi Hotspot and BLE, on the other hand, can be easily employed for OppNets in people-centric smart mobile applications.

These onerous interfaces and their protocols complicate the development of simple and lightweight connectivity and routing services for OppNets. The next section presents how the existing related systems utilize these interfaces for their OppNet services.

TABLE 2.3: Wireless Adapter Incompatibility Examples

\begin{tabular}{lll}
\hline Type & Wi-Fi & Bluetooth \\
\hline Frequency mismatch & $\begin{array}{l}\text { In case dual band is not } \\
\text { supported, 2.4GHz mode cannot } \\
\text { discover 5GHz-band chips. }\end{array}$ & $\begin{array}{l}\text { In case dual mode is not } \\
\text { supported, v4.0+ chips cannot } \\
\text { discover older version chips. }\end{array}$ \\
\hline Interface mismatch & $\begin{array}{l}\text { In case chips have and/or lack of } \\
\text { support for Wi-Fi Ad Hoc, Wi-Fi } \\
\text { Direct, and/or Wi-Fi Hotspot. }\end{array}$ & $\begin{array}{l}\text { For all their smart mobile } \\
\text { devices, Apple derecognizes } \\
\text { chips from other vendors. }\end{array}$ \\
\hline Security mismatch & $\begin{array}{l}\text { Connection issues in case chips } \\
\text { have and/or lack of different } \\
\text { certificates such as WEP, WPA, } \\
\text { WPA2. }\end{array}$ & $\begin{array}{l}\text { N/A: Pairing is backward } \\
\text { compatible for v2.1+EDR and } \\
\text { newer versions. }\end{array}$ \\
\hline $\begin{array}{l}\text { Unsupported } \\
\text { data rates }\end{array}$ & $\begin{array}{l}\text { In case chips use different IEEE } \\
\text { 802.11 amendments (a,b,g,n), } \\
\text { transmission is minimized to the } \\
\text { lowest data rate. }\end{array}$ & $\begin{array}{l}\text { In case chips have different } \\
\text { Bluetooth versions, transmission } \\
\text { is minimized to the lowest data } \\
\text { rate. }\end{array}$ \\
\hline
\end{tabular}




\subsection{SySTEMS}

Partially or fully incorporating the concepts of people-centric OppNets, a number of different network architectures and opportunistic connectivity schemes has been suggested. This section gives a review and comparison on these systems including our architecture, Cocoon presented in Chapter 3. In addition, the existing OppNet applications and services presented in this section.

\subsubsection{Network Architectures}

Regarding the domain of opportunistic communications, the pioneer implementations are presented by the Serval project [44] and Haggle project [45]. As recently initiated projects, there are also other works on the realization of opportunistic communications between smart mobile devices. The SCAMPI project [46] presented CAMEO. The Open Garden project [47], the PodNet project [48], and the 7DS project [49] presented their systems with the same names. Additionally, Boldrini et al. proposed a system presented under the SOCIALNETS project [50].

\section{Serval}

The Serval project presents an Android-based opportunistic communication service called the Serval Mesh [51] to sustain infrastructure-less mobile communications when outages occur in cellular infrastructures. On top of the Serval Mesh, the Serval project offers several applications such as MeshMS [52] which provides an opportunistic extension to the global short message services. The Serval Mesh creates a mesh network of smartphones by exploiting Wi-Fi Ad Hoc mode, and is able to connect with desktop computers as well. The communication is principally devised for voice transmissions over smartphones. In order to support this communication, the Serval mesh network utilizes a specific transport protocol based on Servalaugmented IP addresses (SIP). In detail, the mesh network peers broadcast their SIP with each other to support phone calls and without any user intervention. The seamless operation of voice calls is controlled using the Better Approach To Mobile Ad Hoc Networking (B.A.T.M.A.N.) routing protocol $[53,54]$.

\section{Haggle}

Within the Haggle project, an OppNet paradigm called Pocket-Switched Networks (PSNs) [55] is offered. According to the basic definition, a PSN enables opportunistic communications among people carrying mobile devices. The Haggle project initially presented their real-world PSN experiments with $i$ Mote nodes to investigate and model pairwise contacts. In general, mainly for the use of PSNs, Haggle aims at developing a cross-layer OppNet architecture which has support for the well-known desktop and mobile O/S platforms. According to the latest version of the Haggle API (v0.4), the Haggle architecture supports networking over Bluetooth Classic and Wi-Fi Ad Hoc mode, in particular with fully-support for Android and Windows Phone, yet with limited-support for other O/S platforms [56, 57]. 
The Haggle architecture is able to obscure the technological differences of PHY/MAC interfaces utilized as well as to allow applications to operate according to user preferences. That is, users can share interests defined via an asynchronous publish-subscribe system presented on the application layer. Using this system, several systems are presented which adopt the Haggle architecture to realize opportunistic networking systems. The examples include a content sharing service [58], a localization service [59], and further services [60, 61, 62].

\section{CAMEO}

The SCAMPI project presents CAMEO [63] as their middleware service with the utilization of Wi-Fi Direct on Android platforms. CAMEO primarily focuses on their design presented on top of the transport and application layers, and leaves out dealing with the data forwarding issues of Wi-Fi Direct in large-scale deployments. The reason is that the focus of the project is to collect and reason upon multidimensional context information derived by smart mobile devices and their affiliated users. To this end, CAMEO does not involve a performance study on their proposed networking concept.

\section{Boldrini et al.}

Within the SOCIALNETS project, Boldrini et al. in [64] offer a context-aware OppNet middleware architecture for smart mobile platforms. Like CAMEO, the developers of this architecture put their focus on developing a social context model in the transport and application layers. The architecture adopts the Haggle architecture in their network layer. Regarding this adoption, no additional information is provided to explain how the networking is achieved.

\section{Open Garden}

The Open Garden project presents a closed-source architecture, called Open Garden, that is developed for Android and $i O S$ platforms. The aim of Open Garden is to provide opportunistic communication services among smartphone users by eliminating the user intervention. The architecture utilizes Wi-Fi Direct and BLE for P2P networking, and proposes a routing mechanism based on user profiles.

\section{DS}

The 7DS project proposes an architecture that sets specific abstractions for mobility-oriented social networking designed in the transport layer [65]. Nevertheless, it does not include schemes for solving the problems related to OppNets.

\section{PodNet}

The PodNet project introduces an architecture for opportunistic podcasting [66]. The architecture is positioned mainly over the MAC layer and transport layer to support delay-tolerant broadcast of podcast data (a form of multimedia series) between mobile devices. Additionally, a sync manager is introduced in the application layer to support delay-tolerant transmissions. The PodNet system is principally intended for the use of podcasting in mobile ad hoc networks. Therefore, their solution does not include a connectivity scheme for OppNets. 


\subsubsection{Connectivity Schemes}

It is evident that the majority of the above-presented systems takes the available WLAN and WPAN interfaces "as-is" without considering how connectivity issues can be overcome under the dynamic conditions of OppNets. As earlier discussed in Section 2.2.2, opportunistic networking does not only necessitate a delay-tolerant service, but also demands for adaptiveness in terms of connectivity. Furthermore, the available interfaces show limitations for large deployments as well as for public availability. To overcome these connectivity issues in OppNets, the following solutions are proposed recently:

\section{WiFi-Opp}

Trifunovic et al. proposed WiFi-Opp [41] which offers universal ad hoc support between mobile devices with the alternating use of Wi-Fi Infrastructure and Wi-Fi Hotspot modes. WiFiOpp is a self-organizing connectivity scheme. Since the first time it is presented, WiFi-Opp collected a special attention in the research field for the following reasons:

- It provides an active adaptation of the participating devices against topology changes. It presents a randomization mechanism to dynamically cluster the clients with an AP while evenly distributing energy consumption among the devices.

- It offers a publicly-available ad hoc networking model. Despite its limitations, it resembles Wi-Fi Ad Hoc mode which is not supported in mobile O/S platforms.

\section{Dubois et al.}

The connectivity scheme offered by Dubois et al. is based on an approach adding several improvements for WiFi-Opp [31]. Like WiFi-Opp, the approach provides an adaptiveness in the connectivity reconfigurations. Unlike WiFi-Opp, on the other hand, the approach is able to automatically adjust the durations set for the Wi-Fi Hotspot and Wi-Fi Infrastructure modes in every device based on the changes in channel frequencies and network traffic. Nevertheless, the modifications offered in the MAC layer decreases its public availability.

\section{MA-Fi}

Mobile Ad Hoc Wi-Fi (MA-Fi) presented by Wirtz et al. is another ad hoc networking approach in which devices create self-configuring mesh networks using alternating switches between Wi-Fi Hotspot and Wi-Fi Infrastructure modes [67]. MA-Fi is mainly suitable for MANETs, and the routing algorithm presented on top of MA-Fi is based on IP routing. Therefore, its use in opportunistic communications remains as a question.

\section{Help Beacons}

Al Akkad et al. present a lightweight opportunistic emergency messaging system utilizing the Wi-Fi Hotspot SSIDs as metadata sharing between smartphones [68]. Owing to its beacon (SSID) utilization, the system provides an open accessibility to any kind of user. However, the current implementation supports only a single-hop communication between a local cloud and online systems. In order to discover its performance in large-scale deployments, the study should involve a multi-hop and mobility analysis. 


\section{PASA}

Mao et al. present PASA [69] which allows passive broadcast of wireless network short messages. The passive broadcast is based on the advertisement of wireless network identifiers as network packets. PASA is implemented and tested with a real-world network setup consisting of smartphones with Wi-Fi Direct and Bluetooth Classic support.

The communication model of PASA employs a delay-tolerant data exchange protocol similar to our protocol, Opportunistic Beacon Networking (OBN) presented in Chapter 4. The performance of PASA is evaluated only in a mesh network under no mobility. In contrast, OBN is investigated comprehensively with several mobile network setups and device parameters.

\subsubsection{Applications \& Services}

A few number of applications and services exists supporting opportunistic communications for general-public use. Majority of them are proposed for P2P messaging.

The early examples for ad hoc messaging involve simplistic single-hop solutions. Nokia Sensor [70] enables users to detect others in the vicinity via Bluetooth Classic. Similarly, Beacon Friend Finder [71] also uses classic Bluetooth signals to send out identifiable user messages. The problem of Bluetooth device discovery is the frequent and time-consuming user intervention for device pairing. Eliminating the manual pairing process, E-Smalltalker [72] proposes encoding of Bluetooth device names to enable user-defined content sharing between peers. Similarly, MDSRoB [73] introduces Bluetooth network name encoding for the dissemination of messages inside a Bluetooth piconet. A similar implementation is also presented in [74] for Bluetooth and BLE. However, these studies do not study networking performance.

As recent proposals, E-Shadow [75] and Help Beacons (presented as a smartphone application of the previously-presented Help Beacons scheme) [68] extend the P2P messaging in social spaces via user contents advertised in IEEE 802.11 SSID fields.

FireChat [76] introduced by the Open Garden project is an ad hoc networking application commercially available on smartphones. FireChat makes use of either Wi-Fi Direct or BLE. The networking of FireChat uses fixed Wi-Fi APs in case of necessity for increased scalability.

Figure 2.3 shows the screenshots from the most-recognized ad hoc networking applications introduced for smart mobile devices.

\subsubsection{Beacon Profiles}

It could be observed that beacon utilization in smart mobile applications is gaining popularity. In order to enable interaction between beacon-enabled devices, beacon profiles are used in application layer services. The aim of beacon profiling is to make small wireless sensor devices identifiable with any location or object assigned to them. Beacon profiles take advantage of advertising mode of a wireless radio to broadcast data out in periodic, specially formatted advertising packets. Having received and interpreted by smart mobile devices, these profiles can differently encoded depending on application types. Encoding types include information related to personalized data such as proximity to nearby locations or objects, object recognition, 


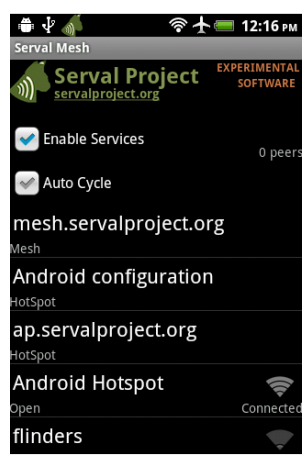

(a) Serval Mesh

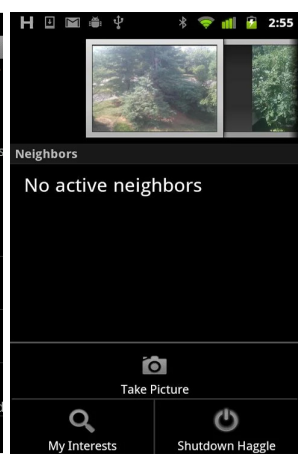

(b) Haggle

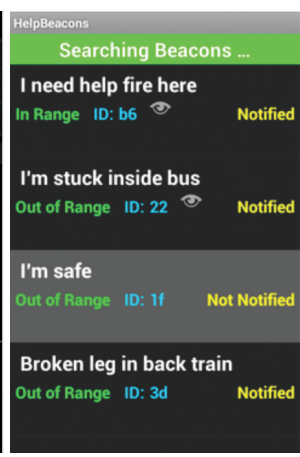

(c) Help Beacons

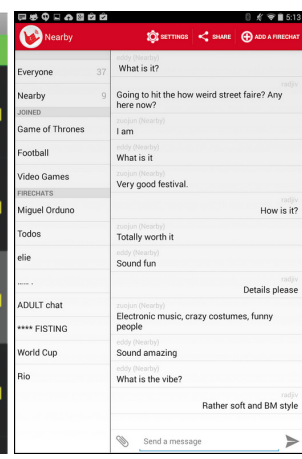

(d) FireChat

Several ad hoc networking applications introduced for smart mobile devices

ownership, temperature, and motion. These profiles can also be utilized for opportunistic communications by supporting beacon encoding with multi-hop routing information. To the best of our knowledge, there is still no example for this category of applications and services. This thesis presents the first example of beacon message encoding, called opportunistic beacons (described in Chapter 3), for the purpose of routing in OppNets.

Recently, several encoding specifications have been offered for beacon-enabled applications and services. The most-known examples are presented for BLE. In 2013, iBeacon is presented by Apple as the first BLE profile specification [77]. In 2014, AltBeacon is presented as a cross-platform encoding BLE standard [78]. In 2015, EddyStone is presented by Google as their generic BLE profile specification [79]. In addition, there are other standards for proprietary uses such as Estimote [80], Gimbal [81], and URIBeacon [82].

Each major standard has significant differences in terms of UUID encoding formatting. Some are open source whereas some are offered with subscription fees. Moreover, their support differs in different mobile $\mathrm{O} / \mathrm{S}$ platforms. While iBeacon is officially supported by $i O S$ devices only, Eddystone and AltBeacon have support for both iOS and Android. While iBeacon and AltBeacon have only one data packet type (identifier frames), Eddystone is designed to support multiple data frame types such as URL, identifier, and telemetry. iBeacon and AltBeacon are very similar in terms of their formats. Eddystone and URIBeacon, on the other hand, have completely different formats than others. Such differences are disincentive for the development of general-purpose smart mobile applications. To provide generic and flexible formats in beacon-enabled applications, ChitChat in [83] is the only BLE profiling example so far. On the other hand, Help Beacons [68] and PASA [69] utilize Wi-Fi SSID for beacon profiling. Nevertheless, these examples can be used only for single-hop device-to-device interactions. All of them are designed for one-way communication methods, and require an external database to store the service definitions. On the other hand, our implementation of opportunistic beacons supports two-way communication between devices with generic, opensource routing specifications defined under the Cocoon architecture. 


\subsubsection{Discussion}

Including our network architecture, Cocoon presented in Chapter 3, Figure 2.4 summarizes the above-referred implementations by showing their focus of study on the generalized protocol stack. Furthermore, Table 2.4 shows a brief comparison between them based on the features they utilize and offer.

It is obvious that the current proposals cannot completely fulfill the requirements of opportunistic networking requirements. OppNet implementations aimed for community-oriented applications must generally accord with large-scale deployments. Such deployments are often formed with disjoint networks of people. To this respect, opportunistic communications necessitate multi-hop networking with mobility assistance. In terms of networking, majority of the existing systems cannot overcome the problem of self-configuration due to mobility. The connectivity schemes based on connections, either the default ones such as Wi-Fi Ad Hoc mode or devised ones such as WiFi-Opp, employed in OppNets reflect the following problems:

i) They face high overhead of network discovery in mobile environments. For OppNets, PHY/MAC layer protocols must provide constant awareness for the contact opportunities, then sustain dependable connections, and finally lead up to reliable/rapid information delivery.

ii) They are bound to small-scale deployments due to the limitations of Wi-Fi and Bluetooth interfaces. An OppNet can yield high performance and scalability only if the wireless interface utilized is tolerant to rapid topology changes.

On the other hand, the connectivity schemes based on connection-free beacon advertisements reflect the following problems:

i) Their throughput is quite limited since they utilize short wireless network identifiers.

ii) Beacon delivery is one-directional, i.e. from a beaconing device to a listening device.

The discrepancy between the lack of support for ad hoc networks and the lack of functionality in infrastructure-based networks is the key challenge for the development of communityoriented networks. To this respect, beacon utilization can be a strong approach to overcome the challenges reflected by mobility and density variations.

The Cocoon architecture utilizes the widely-available Wi-Fi Hotspot and BLE interfaces for increased public availability, and makes use of beacons to address the challenges presented in this section. Besides, Cocoon can be readily integrated to any group and type of smart mobile devices. As a connection-free model, Cocoon's OBN completes data delivery at the neighbor discovery stage, and thus provides high flexibility of data sharing even in highly-dense and highly-mobile networks. 


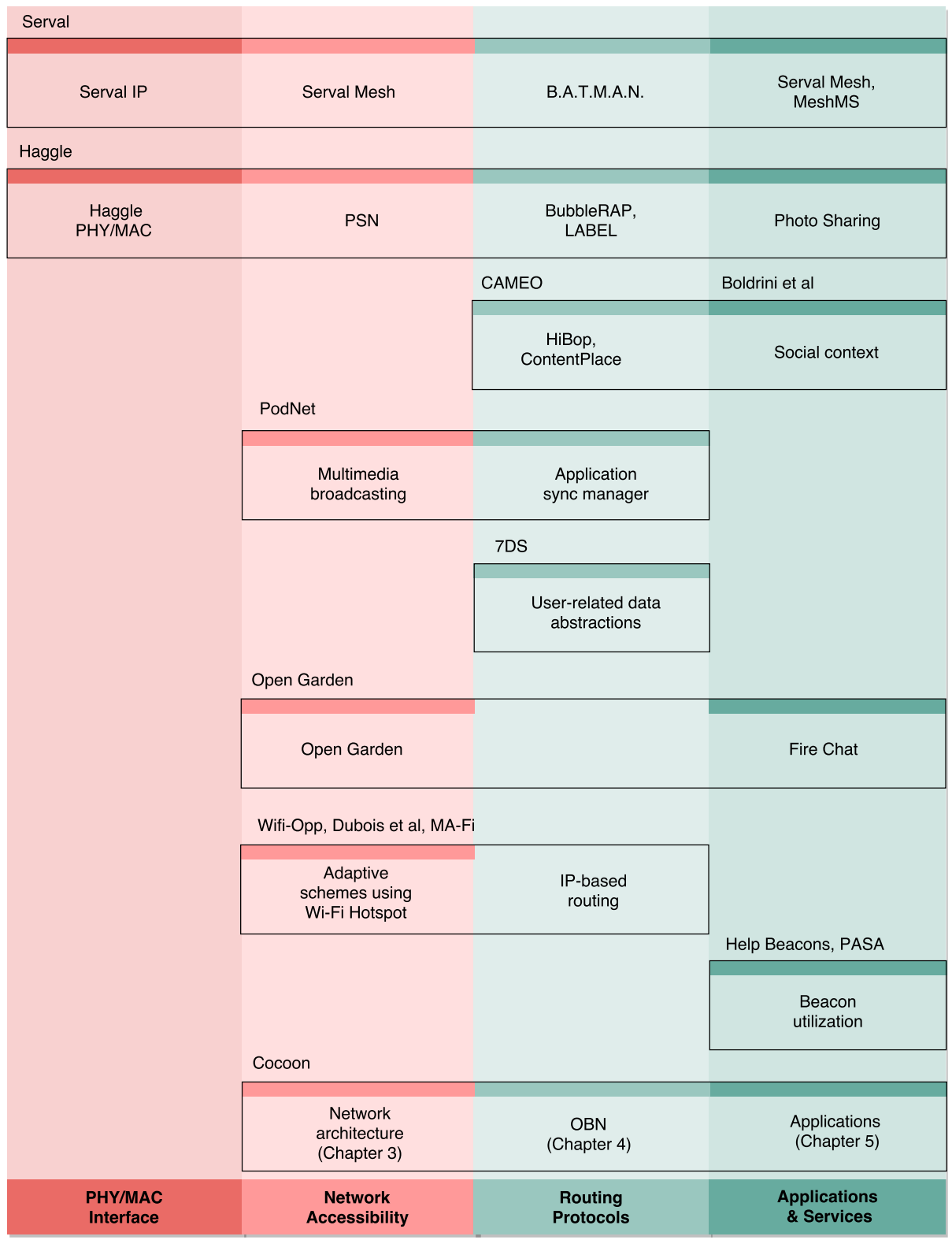

Summary of the existing OppNet systems 

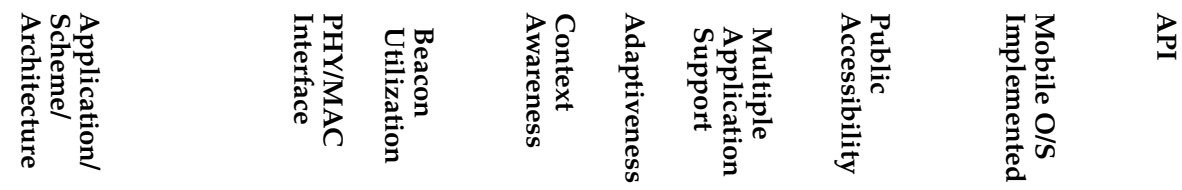

\begin{tabular}{|c|c|c|c|c|c|c|c|c|}
\hline Serval & Wi-Fi Ad Hoc & $x$ & Routing & $x$ & $\checkmark$ & $\begin{array}{l}\text { Root- } \\
\text { Access }\end{array}$ & Android & $\begin{array}{l}\text { Open } \\
\text { Source }\end{array}$ \\
\hline $\begin{array}{l}\text { Haggle } \\
\text { v0.4 }\end{array}$ & $\begin{array}{r}\text { Wi-Fi Ad Hoc } \\
\text { Bluetooth }\end{array}$ & $x$ & Routing & $x$ & $\checkmark$ & $\begin{array}{r}\text { Root } \\
\text { Access }\end{array}$ & $\begin{array}{r}\text { Android } \\
\text { Windows } \\
\text { Mobile } \\
\text { iOS }\end{array}$ & $\begin{array}{l}\text { Open } \\
\text { Source }\end{array}$ \\
\hline CAMEO & Wi-Fi Direct & $x$ & $\begin{array}{r}\text { Routing } \\
\text { Social } \\
\text { Sensors }\end{array}$ & $x$ & $\checkmark$ & $\begin{array}{r}\text { Lim- } \\
\text { ited } \\
\text { Access }\end{array}$ & Android & $\begin{array}{c}\text { Docu- } \\
\text { men- } \\
\text { tation }\end{array}$ \\
\hline PodNet & Wi-Fi Ad Hoc & $x$ & Routing & $\checkmark$ & $\checkmark$ & $\begin{array}{r}\text { Root } \\
\text { Access }\end{array}$ & $\begin{array}{r}\text { Windows } \\
\text { Phone }\end{array}$ & $\mathrm{N} / \mathrm{A}$ \\
\hline 7DS & Not provided & $x$ & Routing & $x$ & $x$ & $\begin{array}{r}\text { Not } \\
\text { known }\end{array}$ & $\begin{array}{r}\text { Not } \\
\text { provided }\end{array}$ & $\mathrm{N} / \mathrm{A}$ \\
\hline $\begin{array}{l}\text { Boldrini } \\
\text { et al. }\end{array}$ & $\begin{array}{r}\text { Wi-Fi Ad Hoc } \\
\text { Bluetooth }\end{array}$ & $x$ & $\begin{array}{r}\text { Social } \\
\text { Device } \\
\text { Routing }\end{array}$ & $\checkmark$ & $\checkmark$ & $\begin{array}{r}\text { Root } \\
\text { Access }\end{array}$ & $\begin{array}{l}\text { Android } \\
\text { \& Haggle }\end{array}$ & $\mathrm{N} / \mathrm{A}$ \\
\hline $\begin{array}{l}\text { Open } \\
\text { Garden }\end{array}$ & $\begin{array}{r}\text { Wi-Fi Direct } \\
\text { BLE }\end{array}$ & $x$ & Social & $x$ & $\checkmark$ & $\begin{array}{r}\text { Lim- } \\
\text { ited } \\
\text { Access }\end{array}$ & $\begin{array}{r}\text { Android } \\
\text { iOS }\end{array}$ & $\begin{array}{l}\text { Closed } \\
\text { Source }\end{array}$ \\
\hline $\begin{array}{l}\text { WiFi- } \\
\text { Opp }\end{array}$ & Wi-Fi Hotspot & $x$ & $\begin{array}{r}\text { Routing } \\
\text { Device }\end{array}$ & $\checkmark$ & $x$ & $\begin{array}{l}\text { Open } \\
\text { Access }\end{array}$ & Android & $\mathrm{N} / \mathrm{A}$ \\
\hline $\begin{array}{l}\text { Dubois } \\
\text { et al. }\end{array}$ & Wi-Fi Hotspot & $x$ & $\begin{array}{r}\text { Routing } \\
\text { Device }\end{array}$ & $\checkmark$ & $x$ & $\begin{array}{r}\text { Open } \\
\text { Access }\end{array}$ & Android & $\mathrm{N} / \mathrm{A}$ \\
\hline $\begin{array}{l}\text { Help } \\
\text { Beacons, } \\
\text { PASA }\end{array}$ & Wi-Fi Hotspot & $\checkmark$ & $x$ & $x$ & $x$ & $\begin{array}{l}\text { Open } \\
\text { Access }\end{array}$ & Android & $\mathrm{N} / \mathrm{A}$ \\
\hline
\end{tabular}

\begin{tabular}{|c|c|c|c|c|c|c|c|}
\hline Cocoon & $\begin{array}{r}\text { Wi-Fi Hotspot } \\
\text { Bluetooth } \\
\text { BLE }\end{array}$ & $\checkmark$ & $\begin{array}{l}\text { Routing } \\
\text { Sensors }\end{array}$ & $\checkmark$ & $\checkmark$ & $\begin{array}{r}\text { Open } \\
\text { Access }\end{array}$ & Android \\
\hline
\end{tabular}




\subsection{Forwarding Protocols}

In OppNets, routing differs from the traditional approaches. Destination is often unknown in terms of locality, routes are generally broken and non-reproducible because of separated and dynamic links between devices. ${ }^{1}$ As a result, forwarding techniques are employed instead of routing techniques. ${ }^{2}$

Forwarding ${ }^{3}$ is coupled with replication to increase delivery likelihood, i.e. a message is forwarded to multiple contacts to increase the possibility of reaching a destination. Nevertheless, forwarding has to be selective for the following reasons:

- To provide efficiency in resource utilization. Excessive message replicas may severely affect shared wireless channels as well as memory/storage of devices. As the number of messages increase in a device, storing them gets difficult.

- To prevent message contentions. As their replicas increase in the network, messages get higher waiting times. Not all of the messages can be forwarded in case of short communication windows.

The ultimate goal of forwarding is to select only the set with the minimum number of devices to forward a message. Forwarding in a device can be designed to accept or reject a message based on a decision-making mechanism. The decision-making can be based on various context or focus of interest. This section presents a comprehensive overview and analysis of several forwarding approaches in OppNets.

In the context of OppNets, forwarding approaches can be categorized in many different ways according to their replication strategies. The following presents two classes of categorization. The first categorization is provided to explain how replication works, the latter is to properly define the problem of context utilization in forwarding.

\section{First categorization: Replication Awareness}

The first categorization is based on how forwarding is controlled in terms of the number of replicas created. The widely-accepted categorization in the research domain consists of three categories: i) single-copy methods, that replicate only one copy of a message, ii) flooding methods, without any form of suppression and iii) controlled-flooding methods, that assign a limit to each message or device for replication.

1. This thesis regards opportunistic forwarding as a mechanism that works over intermittent and opportunistic contacts and omits forwarding based on other types of contacts, such as scheduled contacts. An opportunistic forwarding protocol mainly operates based on unknown route information, i.e., without the network topology information.

2. Routing and forwarding are interchangeably-used terms in the research domain.

3. Henceforth in the thesis, opportunistic forwarding will be referred to as forwarding. 
Single-copy methods are beneficial when the network resources are scarce [84]. In such environments, single-copy forwarding helps to avoid issues arising from congestion and energy utilization. The simplest single-copy approach is called direct delivery, in which a device forwards a message only to the contact that is the destination. The second approach is called first contact, in which a device forwards a message only to the first contact regardless the contact is destination or not.

Flooding methods are based on greedy replication strategies. The widely-known example for flooding is Epidemic routing [85], which utilizes a summary table for the messages in each device. When devices meet each other, they first exchange their summary tables in order to prepare the messages which are missing at the other side of the connection, then start forwarding the copies of those messages between each other. The uncontrollable number of copies in flooding strategies may cause data congestions.

Controlled-flooding methods stipulate conditions on replication. These conditions are often based on a threshold set for the number of message copies. Spray $\mathcal{E}$ Wait [86] and Spray \& Focus [87] are the widely-known examples for semi-flooding, in which the number of message relays are set to a limit (threshold). Spray \& Wait has two phases: In the first phase, for each message, a device distributes a fixed number of copies to the first contacts encountered. In the second phase, when the threshold set for the number of copies is reached for a message, the device suspends forwarding it until the destination is encountered. The second phase of Spray \& Wait is problematic especially in dense environments where in the first place the messages can only be spread to a limited area far from the destination. Spray \& Focus introduces a single-copy forwarding approach for the second phase to increase scalability.

Devising utility functions based on the observations in inter-contact relations prudently assists replication control. The most widely-known examples of utility-based forwarding are MaxProp [88], PRoPHET [89], RAPID [90], and EBR [91] which provide selectiveness by utilizing various contact information such as encounter history and message delivery statistics. Together with other types of examples, they will be discussed in our second categorization.

\section{Second categorization: Context Awareness}

This thesis offers a new categorization for forwarding methods based on the context source types utilized in an OppNet. This categorization is novel to show the following considerations in forwarding design:

- What are the context sources that can be used in forwarding decisions?

- How can the context sources be obtained in OppNets?

- What are the effects of the context sources on forwarding performance?

As Jain et al. revealed in [92], context utilization makes forwarding decisions more attentive on the network conditions. Unlike stochastic replication methods, context-aware methods aims at developing adaptable forwarding decisions based on information about a contact, a situation, or network characteristics. The context sources are classified into three groups: 
Encounter context, which includes information gathered from contacts, such as interdevice similarities and information about other devices. Such information accumulates over time, but is subject to lose actuality within time.

Social context, which includes a previously-known or predicted information about a user regarding his/her social status or dependencies, such as user profile, social ratings, and popularity. Like encounter context, the actuality of social context can be lost within time.

Self-generated context, which includes information generated by observations or measurements such as schedules, contacts that are encountered, points of interest, mobility routines, physical activities, and environmental status. Self-generated context can be beneficial to make an inference about a situation or contact in the first place. Moreover, long-term observations can be helpful to predict the future.

Given the context sources, information can be collected by either self observations or from encounters. Besides, foreknown information such as social context can be utilized in forwarding decisions.

The aim of utilizing encounter context in forwarding is to detect similarities (or dissimilarities) between contacts. The previously-explained controlled-flooding approaches are the simplest examples that utilize encounter context. As one of the earliest studies exploiting pairwise node similarities, MobySpace [93] investigates similarities in device mobility patterns. This approach allows forwarding between contacts having the similar predicted destinations. As a similar method, Profile-Cast [94] analyzes contact similarities with respect to mobility traces and profile information. In this approach, devices exchange the summary of their mobility profiles to decide whether they show high similarity to continue message switching. As a periodicity-aware strategy, Predict and Relay [95] constructs an algorithm to predict the likelihood of future inter-contact times based on semi-deterministic mobility patterns. The approach presented in [96] gives a detailed analysis on understanding periodicity in inter-contact times by utilizing both mobility and encounter traces. Similarly in [97], authors aim to discover periodic patterns of encounters. As a similar strategy, dLife presented in [98] exploits pairwise relations by detecting daily patterns based on proximity graphs inferred directly from inter-contact times. On the other hand, the methods presented in [99] and [100] state that dissociations between contacts can be utilized to improve forwarding performance.

Another motivation in the development of forwarding strategies is to exploit social context to map contact opportunities with the foreknown, predicted, or measured social metrics. Recently, a great number of forwarding methods have been proposed that utilize various information related to social relationships between contacts. The pioneer examples such as Label [101], SimBET [102], and PeopleRank [103] exploit social dependency information of network devices to generate forwarding groups. In addition, BubbleRAP [104] has proved that utilizing several graph theory measures such as node centrality and betweenness may increase forwarding performance. One one hand, it would seem advantageous to exploit social context. On the other hand, however, social relationships among users are ever-changing; i.e. obtaining an up-to-date social context is costly and often impossible. 
Utilizing self-generated context in forwarding decisions can be beneficial to readily react to network topology changes. Concentrating on self-generated context, various offline algorithms exist in the research field of ubiquitous computing. The examples include, but not limited to, the analysis of mobility traces [105], estimation on human activities and behaviors [106], periodicity forecasting [107], next-place prediction [108, 109, 110]. In order to utilize such algorithms in forwarding strategies, they must be adapted for on-the-fly scenarios.

Furthermore, forwarding methods can be coupled with situation awareness. The role of situation awareness is to keep a shared, actualized, extensive, and reliable picture of a current situation happening in an environment. This can allow to adaptively make "on-the-go" forwarding decisions by understanding or anticipating the rapid changes in an environment. However, developing a situation-aware tool is a non-trivial and costly task which necessitates constant observations and measurements performed in the network [111]. Such studies fall under the research field of opportunistic sensing, which is not taken as a research focus in this thesis. 


\subsection{Concluding Remarks}

By and large, the mobility assistance greatly needed by OppNets requires innovative design considerations for opportunistic communications with smart mobile devices. While having promising potentials, the current wireless standards (e.g. Wi-Fi, Bluetooth) have restricted or hidden support for ad hoc communications in mobile operating systems. Besides, these standards are designed to achieve ad hoc communications under stable connectivity, therefore cannot cope with the highly-dynamic characteristics of OppNets. The research field of OppNets is struggling in finding new ways to eliminate those limitations and challenges. Recently, beacon utilization is found to be a good strategy to pose the difficulties in connectivity maintenance between and among mobile devices. Moreover, beacon utilization provides high availability, i.e. all kinds of devices support beacons regardless of the inconsistencies reflected by different wireless radios and affiliated adapters. However, the appropriateness of beacon utilization for opportunistic communications is still not widely investigated. The existing approaches that exploit beacons for opportunistic communications are quite limited to provide a reasonable networking.

Presenting different types of context awareness, the forwarding strategies mainly focus on the user-related information to find ways for an optimized routing with the help of user's daily mobility patterns. Nevertheless, such social metrics are difficult to obtain, to update, and to utilize. For instance, especially in challenged environments, the mobility patterns of participating devices (users) may not match with the reality, i.e. unexpected patterns that are quite likely to happen can negatively affect forwarding performance. Furthermore, the actuality of the context may be outdated very quickly. In conclusion, it is possible to state that the forwarding performance is not directly proportional with the context used under different circumstances. 



\section{Community-Oriented Context-Aware Opportunistic Networking Architecture}

THIS CHAPTER PRESENTS THE GENERAL ARCHITECTURE OF OUR COMMUNITYoriented context-aware OppNet architecture, called as Cocoon. COCOON COMPRISES A SET OF OPPORTUNISTIC COMMUNICATION SPECIFICATIONS DEVELOPED FOR SMART MOBILE PLATFORMS. IT PROVIDES A MOBILE O/S SYSTEM FOR SUPPORTING LIGHTWEIGHT AND UNIVERSAL OPPNET APPLICATIONS THAT ARE COMPATIBLE AND FREE TO ENGAGE WITH ANY OTHER KIND OF MOBILE SYSTEMS. WITHOUT REQUIRING SOPHISTICATED CONFIGURATIONS, ROOT ACCESS, OR KERNEL/DRIVER MODIFICATIONS, IT CAN BE USED IN A WIDE VARIETY OF MOBILE PLATFORMS FROM SMARTPHONES AND SMARTWATCHES TO TABLETS AND LAPTOP PCS THAT SUPPORT WIRELESS SHORT-RANGE INTERFACES.

Of the Cocoon architecture, the functionalities Related to THE CONNECTIVITY MANAGEMENT, NETWORKING, AND SERVICE MANAGEMENTS ARE DISCLOSED. THE CONNECTIVITY MANAGEMENT IS PROVIDED WITH A NOVEL OPPORTUNISTIC SCHEME CALLED AS OPPORTUNISTIC BEACONS. IN SHORT, OPPORTUNISTIC BEACONS UTILIZE WIRELESS NETWORK IDENTIFIERS TO FURTHER PROVIDE NETWORKING AND QUALITY OF SERVICE (QOS). FOR NETWORKING, THE COCOON ARCHITECTURE EMPLOYS TWO DIFFERENT NETWORKING MODES: THE FIRST ONE IS A CONNECTION-FREE MODEL SOLELY BASED ON INFORMATION SWITCHING VIA OPPORTUNISTIC BEACONS. THE SECOND ONE IS A CONNECTIONBASED MODEL UTILIZING OPPORTUNISTIC BEACONS TO REGULATE NETWORKING AMONG DEVICES. For QOS, OPPORTUNISTIC BEACONS PROVIDE GROUPS OF DEVICES TO DISTRIBUTIVELY MANAGE THEIR RUNNING APPLICATIONS. THE APPLICABILITY OF OPPORTUNISTIC BEACONS IS STUDIED WITH A SET OF REAL-WORLD SMARTPHONE EXPERIMENTS. 


\subsection{Overview}

Before going into the details of the Cocoon architecture, this section unveils how the system services are characterized in consideration of the system requirements. Furthermore, the basic components and functionalities of the architecture are briefly introduced.

\subsubsection{System Requirements}

It is needed to define the requirements for the development of the architecture before being able to exploit all the capacities of available technologies for OppNets. The realization of OppNets imposes many challenges. It is in the first place to cope with the challenges related to the development. The design must accord with the used technology. Secondarily, providing a feasible operation is another question of the development.

\section{Design Requirements}

As previously stated in Chapter 2, the limitations and restrictions reflected by today's mobile $\mathrm{O} / \mathrm{S}$ platforms dictate a careful design on the affiliated wireless interfaces. The interfaces of Wi-Fi and Bluetooth are provisioned either as hidden features or with limited functionality. On the other hand, they are not strong enough to provide networking service under topology variations. The inference drawn from here is that ad hoc connectivity to be used for opportunistic communications with the interfaces of either Wi-Fi or Bluetooth is insufficient. For a dependable connectivity between devices, all that is left is to exploit wireless network identifiers (simply beacons) as messages shared in an OppNet. The existing approaches that resort to beacon utilization are very limited in terms of providing a serviceable connectivity, and they do not take the aforementioned challenges into consideration. Therefore, there is still a need for a persevering approach that can make use of beacons for several opportunistic communication purposes.

Another requirement is providing an ease of use. To make every possible device (user) involved in the process of any type of opportunistic communications, a universally-compatible networking model is a must. That is, the proposed network protocols have to be suitable for any type of device and affiliated radio. Again, beacon utilization can be a good candidate to grant compatibility in networking.

In consideration of these requirements, the Cocoon architecture takes care of connectivity and opportunistic networking with a set of beacon-enabled protocols. Despite their advantages, beacons per se represent several shortcomings as well. To cope with these shortcomings, the proposed protocols are harmonized with smart beacon encodings. Section 3.2.1 describes the basics of how beacons are utilized in the architecture. 


\section{Operational Requirements}

Having the general requirements given in Chapter 1, OppNets have to be self-configuring, self-organizing, and self-healing networks. The existing wireless technology on smart mobile platforms creates another set of difficulties to perform networking with high scalability and adaptability in view of highly-dynamic characteristics of OppNets.

In the realm of opportunistic communications, many researches focuses on the characterization of contacts $[112,113,114,115]$ to cope with random topology changes. From a different perspective, some studies have focused on utilizing other available information such as vicinity properties (close-by neighbors, strangers, etc.) [116, 117, 118] and social properties (user popularity, social status, etc.) [64, 119]. An interesting observation in related proposals is that the individual requirements of each device (or user) has not been studied well. While each device, user, or application belonging to an OppNet tries to help achieve optimal system performance, each may have an individual requirement over their own share of overall performance. Cocoon introduces well-defined multi-criteria objectives for this requirement in Section 3.4.1.

\subsubsection{System Components}

The core of the Cocoon architecture is based on the connectivity management, networking, and service management components.

\section{Connectivity Management}

The connectivity management of Cocoon can run on top of the universal interfaces without violating standards. Thus, the proposed routing protocols are compatible with any type of smart mobile device. Within the architecture, the concept of opportunistic beacons (OBs) is introduced. By its literal definition, an OB is a WLAN or WPAN identifier encoded as a network and service management information. At this point, it is worth to mention that an $\mathrm{OB}$ can also carry application-specific information. In networking point of view, an OB is a device in beaconing operation. On the other hand, a device in scanning operation is simply called a beacon observer (BO) which can catch the advertised encoding without orienting device-todevice associations.

To put it simply, simple packet switching and coordination between devices is provided over the built-in advertisement beacons, i.e. human-readable wireless network identifiers. That enables lightweight and instantaneous data forwarding in ad hoc fashion.

$\mathrm{An} \mathrm{OB}$ and a $\mathrm{BO}$ are utilized to provide the basics of networking, service management, and information sharing. An OB is composed of three encoding segments: The first segment includes reserved keys for routing information whereas the second segment includes information about service. Finally, the third segment holds the payload information. In an OppNet, BOs can gather these encodings from the OBs and can adapt themselves according to the provided information. 


\section{Network Management}

One of our hypotheses is that the plethora of devices in a specific area operating as either an $\mathrm{OB}$ or a $\mathrm{BO}$ can form an ad hoc network. The architecture can regulate the information flow in an OppNet with specific OB-BO arrangements. Continuously switching between the $\mathrm{OB}$ and $\mathrm{BO}$ roles, devices can continuously organize opportunistically-formed network groups between each other. Utilizing OBs and BOs, the architecture offers two different networking models. In the first model, called Opportunistic Beacon Networking (OBN), messages are simply shared with the OBs, i.e. the WLAN or WPAN identifiers, e.g. IEEE 802.11 SSID, Bluetooth network name, or BLE UUID. Thus, data exchange can be performed directly with the beaconing and scanning operations without establishing connections. In the second model, called Opportunistic Association Networking (OAN), the OBs are exploited for the establishment of connection-based networks.

Between these two networking models, OBN and OAN, the main interest is given to OBN in this thesis. Individually described in Chapter $4, \mathrm{OBN}$ is a good candidate to provide opportunistic communications in a lightweight and flexible way. OBN is proposed mainly for highlyopportunistic but limited-throughput data dissemination scenarios in which only short messages can be handled. On the contrary, OAN is proposed for small-scale but high-throughput group communications, is not considered as a work of study within the thesis focus, however can still be used in several critical scenarios such as for opportunistic sensing applications based on group communications.

\section{Service Management}

Another hypothesis put forward is that OBs can be utilized to manage networking-specific tasks. For instance, routing can cooperatively be managed between devices with the utilization of routing information encoded in the wireless network identifiers. Besides, applicationspecific information can be used to schedule concurrently running applications in an OppNet environment assuming that devices run multiple applications.

As regards efficient communications, OppNets rely on density and mobility of participating devices. Density and mobility, apart from their advantages, form exceptional challenges that can obstruct network operability. Pertaining to the number of participating devices, devices may face difficulties during neighbor discovery, connection establishment, and transmission phases. On the other hand, the information generated by different sources in an OppNet may increase dramatically, generating a need for resource utilization. Cocoon provides necessary routines for these tasks.

For network-level and application-level operations, a new set of service requirements are defined in consideration of the ever-changing nature of OppNets. These requirements are mathematically modelled as formal decision criteria. They are investigated with several networking examples based on a multi-criteria analysis model. The model runs a periodicallyupdated distributed decision-making algorithm on each device to schedule their applications. The algorithm dispatches the most appropriate application into running state. Devices distributively negotiate which application to run by interchanging information about their current network status. 
The merit of this algorithm is that the metrics proposed for this negotiation process can assess the rapid changes in contacts and shared data. To find and optimize congruent network services, especially in challenging scenarios, the metrics are capable of estimating current network requirements.

\section{Development Interface}

The architecture includes generic service definitions (variables and metrics) and routines that are together presented as an application programming interface (API). The API can assist the progress of OppNet application development on any type of smart mobile platform. Thus, it is readily compatible for any type of smart mobile device.

Figure 3.1 demonstrates the high-level illustration of the Cocoon architecture components and shows the abstract orientation of the API. The API is an instrumental inter-layer in between the application and network layers. It provides an ease-of-applicability to experts in developing Cocoon-based opportunistic communication applications.

As an independent chapter, the Cocoon API is presented in Appendix C.

\subsubsection{Chapter Organization}

The rest of the chapter is organized as follows: Section 3.2 presents the system architecture. In detail, it shows how the networking is handled and what kind of network-specific information is utilized to develop QoS awareness in the networking service. Section 3.3 introduces the fundamentals of OBs. It shows the characteristics of beacon utilization and investigates the applicability of beacons in opportunistic communications with several real-world tests. Section 3.4 describes the QoS model utilized for service management. It describes how the network-specific information is utilized to distributively develop a serviceable OppNet environment. Finally, Section 3.5 presents the concluding remarks.

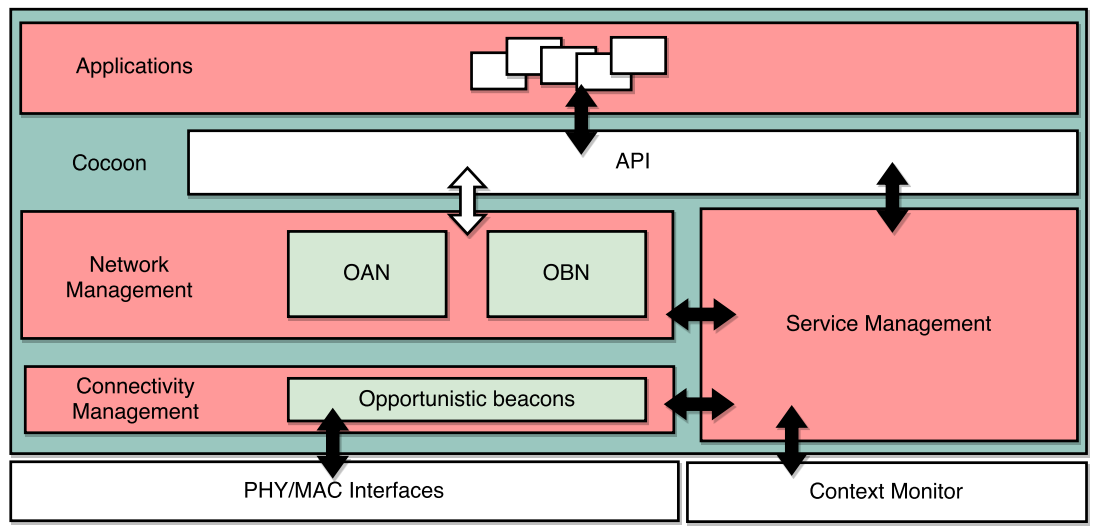

The Cocoon API and system components 


\subsection{System Architecture}

The Cocoon architecture consists of 4 system main managers, given in the following, and their inter-relationship is shown in Figure 3.2.

Network manager (NM) is responsible for connectivity and routing. NM consists of the OBN and OAN models which are discussed in Section 3.2.1.

Context manager (CM) is responsible for the management of context collected from different sources. The context utilized is mainly related to network-specific information, that are routing information and QoS information. The details related to CM are discussed in Section 3.2.2.

Adapter manager (AM) includes the interpreter and timer modules. The responsibility of the interpreter is twofold: i) to decode received messages via the network interfaces, and ii) to encode locally-generated context as messages. Based on the context, the timer module adjusts the timing values used in NM.

QoS Manager (QoSM) runs a distributed scheduling algorithm to manage the concurrently running applications in devices. The details related to QoSM are given in Section 3.2.3.

NM and CM feed each other with incoming and generated data, respectively, via AM. In addition, the architecture employs a background module, context monitor, to convey generated data obtained by $\mathrm{CM}$ routines to AM.

\subsubsection{Opportunistic Networking}

$\mathrm{NM}$ comprises the connectivity routines and runtime variables that are are responsible for providing a mediated access and control to the underlying low-level PHY/MAC interfaces. Utilizing these routines and variables, NM allows for two forwarding strategies:

i) connection-free forwarding, and ii) connection-based forwarding.

FigURE 3.2

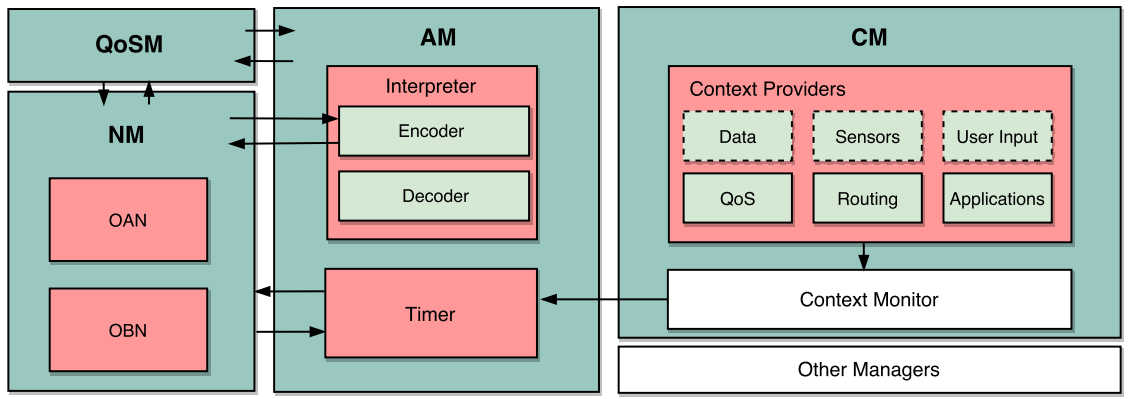

System Architecture 
In both strategies, the data switching mechanism is delay-tolerant, i.e. sending and receiving data between participating devices resist to disconnections. In other words, data transmissions are provided with a store-carry-forward fashion.

Connection-free forwarding is mainly developed for data dissemination scenarios, and its general networking model is introduced as Opportunistic Beacon Networking (OBN). As will be discussed later in Chapter 4, OBN can also be utilized for point-to-point networking. On the other hand, connection-based forwarding is mainly intended for group communications to support publish \& subscribe oriented data streaming applications, and its general networking model is called Opportunistic Association Networking (OAN).

In OBN and OAN, devices can operate in two wireless modes:

i) beacon mode to advertise a specific packet as its WLAN or WPAN identifier.

ii) scan mode to collect the WLAN or WPAN identifier(s) in their proximity.

A device generating beacons under the control of the Cocoon architecture is called an Opportunistic Beacon (OB). On the contrary, a device in wireless scan mode is called a Beacon Observer (BO). In OBN, an OB is exploited as a data packet which can be directly received by the $\mathrm{BOs}$ in its proximity without connection establishment. In OAN, an OB is exploited as a potential network provider available to support connection-oriented data transmissions with the BOs in its proximity. Figure 3.3 shows the data flow operations of OBN and OAN over an illustrated network.

From an $\mathrm{OB}$ to a $\mathrm{BO}$, a packet transmission is one-directional. The reason is that wireless adapters cannot handle the wireless beaconing and scanning operations at the same time. As a consequence, devices running in same modes, i.e. all as OBs or all as BOs, cannot discover each other. In order to increase the possibility of device-to-device discoveries, NM utilizes an automaton that generates switches between the $\mathrm{OB}$ and $\mathrm{BO}$ modes.

As illustrated in Figure 3.4, the automaton has three states corresponding to three discrete wireless modes: $\mathrm{OB}, \mathrm{BO}$, and an idle transition mode between $\mathrm{OB}$ and $\mathrm{BO}$ which is called Update \& Switch (U\&S). During a transition to OB, the utilized WLAN or WPAN identifiers are overloaded with new information at U\&S state.
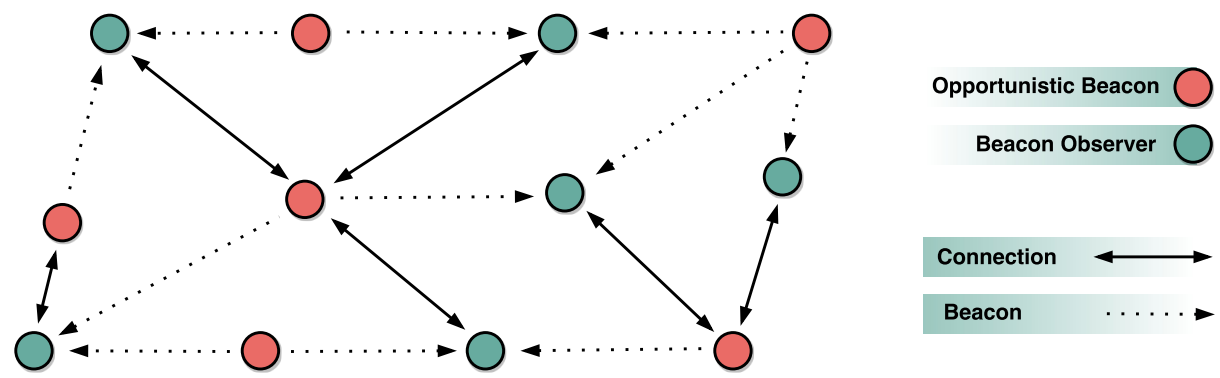

An illustration of OB-BO arrangements 


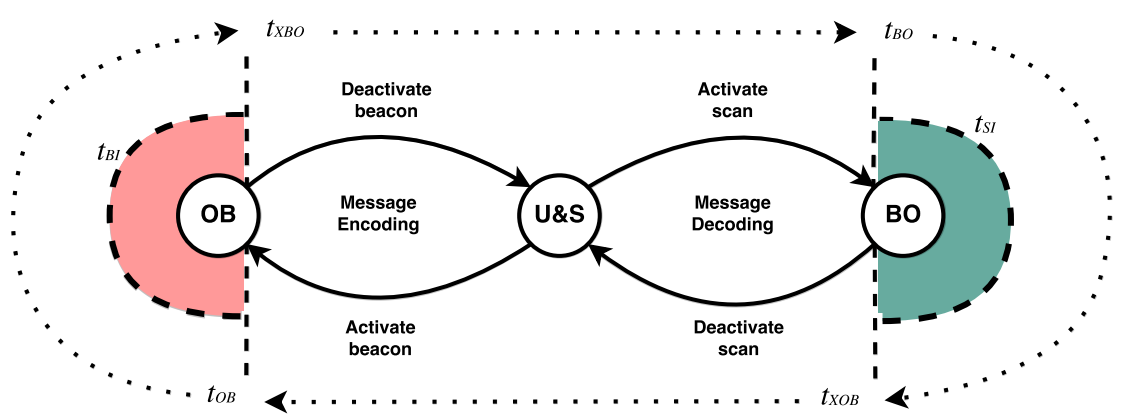

State Diagram for Networking Support

Running the automaton with a random initialization time, a device can be in either of these three states at a particular time. Each state of the automaton has specific service durations. The durations of $\mathrm{OB}$ and $\mathrm{BO}$ are $t_{O B}$ and $t_{B O}$, respectively, which can be adjusted based on the network or application needs. At BO state, scan operation repeats in every $t_{S I}$. At OB state, beacon operation repeats transmission of a specific beacon in every $t_{B I}$. At U\&S state, activate and deactivate operations together defines the switching duration. Transition from $\mathrm{OB}$ to $\mathrm{BO}$, i.e. deactivating beaconing and activating scanning has a total duration of $t_{X B O}$. Transition from $\mathrm{BO}$ to $\mathrm{OB}$, i.e. deactivating scanning and activating beaconing takes $t_{X O B}$ in total. As later investigated in Section 3.3.3, both $t_{X B O}$ and $t_{X O B}$ are non-deterministic wireless adapter-specific durations. Therefore, $U \& S$ creates an uncertainty in the duration of a single automaton cycle. The time period of an automaton cycle, $T$, is the sum of $t_{O B}, t_{X B O}$, $t_{B O}$, and $t_{X O B}$, which is therefore non-deterministic as well. Nonetheless, this uncertainty is helpful to reduce the possibility of OB-OB and BO-BO conflicts if $t_{O B}$ is equal to $t_{B O}$.

In $\mathrm{OBN}$, data forwarding can only be performed at $\mathrm{OB}$ state and external context monitoring can only be performed at $\mathrm{BO}$ state. In OAN, data forwarding and context monitoring can work either at $\mathrm{OB}$ or $\mathrm{BO}$ states through established connections.

\subsubsection{ConteXt Utilization}

Context-awareness plays the most crucial role for the efficient regulation of networking operations and fair content distribution. In our architecture, the union of three data types defines the context utilized: $i)$ routing-specific data $\left.\left(I_{R}\right), i i\right)$ QoS-specific data $\left(I_{Q o S}\right)$, and iii) applicationspecific data $\left(I_{A}\right)$. CM utilizes only $I_{R}$ and $I_{Q o S}$ in its service management. In case of necessity, $I_{A}$ data can be integrated to this management with regard to user-level decisions.

$I_{R}$ involves routing-related information such as time-to-live (TTL) and the current number of hops allowed in forwarding, namely hops-to-go (HTG).

$I_{Q o S}$ involves several metrics related to the ever-changing networking characteristics of devices and network data packets. Over time, the availability of network devices may change and there might be changes in the number of network devices. Translated from the observed changes in the wireless network locality, each device periodically updates its local $I_{Q o S}$. 
$I_{Q o S}$ of a device involves several metrics related to its local contact stability, contact diversity, data stability, and data diversity. These metrics are mathematically expressed in Section 3.4, to be further used in the analytic decision-making process run by QoSM. For the sake of example, $I_{Q o S}$ allows the service management to determine whether the overall network conditions are no longer suitable for a particular application and to replace that application with a suitable one. Similarly, the service management may identify $I_{Q o S}$ that previously was not suitable for certain applications, but that has become suitable.

$I_{A}$ can be additionally utilized by application users to provide flexible input to OppNet applications running on top of the architecture. $I_{A}$ involves application type information and the payload. Regarding $I_{A}$, each registered application in the architecture is advertised in an OppNet with a specific constant header. This header defines the application type and holds a string indicating the application requirements. Application users can implicitly provide updates on $I_{A}$ through the payload. If the application provides an additional interpreter for the payload, $I_{A}$ can be utilized to supersede $I_{Q o S}$ and $I_{R}$. The architecture also allows application users to explicitly change application requirements through its API.

\subsubsection{Service Management}

The dynamic nature of OppNets pushes for an optimization requirement for the applications and resources flowing on their participating devices. QoSM is responsible for operating a distributed scheduling scheme for the concurrently running applications in a device so that diversities and rapid changes in network characteristics can be exploited for optimal networking performance as much as possible along with individual requirement for applications being satisfied. The operation is a distributed one based on an opportunistic scheduling of concurrent applications. Each application is characterized with a predefined objective, then the objective is coupled with its current networking requirements. These requirements are announced and updated in every automaton cycle with the following order: Each device running in OB mode advertises the requirements and objectives of its currently running application. The running state of the application continues until the OB mode expires. In BO mode, each device collects all the advertised $\mathrm{OB}$ within its physical proximity, and makes a choice to yield the most appropriate application for the running state.

Each device deals with its own local application scheduling (assuming that it has multiple applications running on it), without regard to what other devices are doing. In general, independent scheduling is not considered as an efficient way for distributed networking scenarios for the following reason: The overall networking between devices might reflect overall performance drop or totally be defunct when each device runs different applications. To eliminate this possibility, applications are given dynamic priorities, i.e. the priority of each application is updated over time. When the application is in the wait queue, the priority value is incremented in every automaton cycle whereas is decreased when the application is in the running state. The applications with higher priority in an OppNet own higher probability to operate together. However, the prioritization mechanism constitutes only one part of our decision-making algorithm. The most influential part of the scheduling relies on developing a trade-off mechanism between network characteristics and application requirements. 


\subsection{Opportunistic Beacons}

A typical wireless communication consists of three stages. The first stage is the neighbor discovery: Devices seek for beacons to find networks. The second stage is the connection: Following up an authentication process, devices associate with each other to establish links. The third stage is the delivery: Devices switch data during their common communication windows. OBs perform data switching at the neighbor discovery state, thus eliminates the requirement of association, authentication, and connection establishment.

Stuffing application- or routing-specific information into the WLAN and WPAN identifiers has been previously studied in several research areas such as for the use of lightweight advertisement techniques in wireless sensor networks applications [120] and to leverage the location-based services [121, 122]. To the best of our knowledge, Cocoon embraces the first opportunistic technique of beacon frame encoding for the use of OppNets. In essence, with special adaptations on the standards, it is possible to modify default fields of the WLAN and WPAN beacon frames, or to add new ones, to increase the content load. However, such adaptations on the default mobile O/S configurations are not recommended for the best PHY/MAC layer operability [12]. Besides, such adaptations impede the public end-use of the related applications since they require low-level configurations. To provide support for highly-available, highly-accessible OppNet applications, Cocoon involves OB encoding solely on the allowable fields of the WLAN/WPAN beacon frames.

This section first describes the characteristics of OBs in detail, then explains how they are encoded as different WLAN/WPAN identifiers, and finally studies their applicability on smart mobile platforms.

\subsubsection{Characteristics}

Until now, almost all of the existing opportunistic techniques used the obvious contacts, i.e. the contacts happening between only two devices have been taken as the basic requirement to start data switching between each other. Contrarily, OBs, as the units of the connectivity scheme, can have multiple contacts. More specifically, the advantage of OBs is that they exploit wireless broadcast advantage, so that multiple OBs can be detected by a single scan operation. Similarly, multiple BOs can receive an OB without any contention problem arising. This advantage can be reflected as large and fast scalability of information.

Utilizing OBs provides other advantages as well. They can smoothly provide connectivity in networks of heterogeneous devices. The inconsistencies reflected by different device specifications, wireless adapter properties are therefore overcome. They eliminate adapterspecific limitations among participating devices. They provide fast data exchange even under high device mobility and density. Even at short periods of contact durations, they allow for instantaneous transmissions.

In other respects, utilizing OBs represent certain challenges. First of all, beaconing and scanning operations are mutually exclusive on the wireless adapters. Therefore, packet forwarding is one-directional—from a beaconing device to scanning devices. As a delay-tolerant 
forwarding, it is subject to potential delays. To overcome this, devices must continuously employ switch cycles between scanning and beaconing. Second, network throughput is extremely low, bound to the beacon frame length, which hinders sending large packets at once. For multipacket advertisements, wireless identifier fields have to be re-encoded in every switch cycle.

\subsubsection{IDENTIFIER ENCODING}

An OB has to be structured succinctly since WLAN and WPAN identifiers have relatively very limited length in comparison to an ordinary network packet. The editable identifier lengths of the Wi-Fi, Bluetooth, and BLE standards are quite limited. By default, Wi-Fi SSID is 32 ASCII bytes, Bluetooth network name is 248 UTF- 8 bytes (However, it is limited to either 20 or 44 UTF- 8 bytes by majority of the mobile O/S platforms), and BLE UUID is 128 bits. In brief for each identifier type, Figure 3.5 shows the generic OB structure which is composed of the header, routing, QoS, and application data fields. For SSID and Bluetooth Network name encoding, Base 94 encoding is utilized to represent 94 printable ASCII characters in the encoding. For UUID, on the other hand, bitwise encoding is applied. The fields are explained in the following:

\section{Header}

A wireless network name is identified with a specific header in order to advertise an OB as a Cocoon network service as well as to make BOs filter out the WLAN/WPAN identifiers generated by unrelated networks. The header holds the following information:

i) The networking objective of the application (e.g. data dissemination or end-to-end routing). 9 different networking objectives can be defined for an application.

ii) The predefined priority of the application (e.g. urgent, high, low, etc.). 10 different priority levels can be defined for an application.

iii) The predefined type of the application (e.g. personal communications, public networking, vehicular networking, etc.). 94 different application types can be defined.

(i) and (ii) are encoded together to form the header identifier. As 90 different combinations are available, the first byte of the SSID field is dedicated for this identification.

The second byte of the SSID field is used to define (iii), i.e. 94 different application types (Same encoding holds for the Bluetooth network name field). For BLE, on the other hand, the first 8 bits are used to encode (i) and (ii) together, and the remaining for (iii).

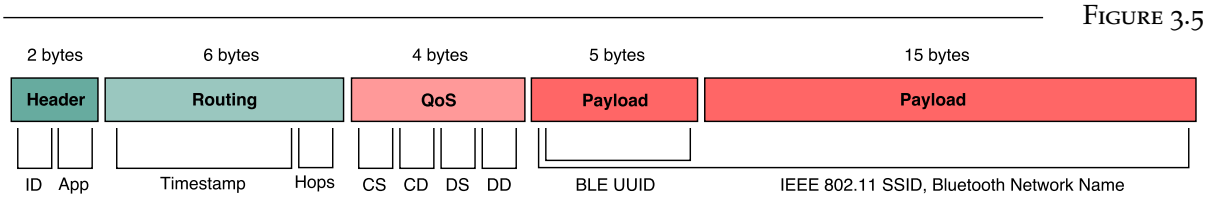

Structure of an opportunistic beacon 


\section{Routing}

In brief, the routing field holds $I_{R}$, more specifically, the information related to the routing of an OB. The advertised $I_{R}$ can be decoded by BOs in proximity for several routing purposes. For instance, the time-to-live (TTL) of an OB or a creation time of a message can be obtained. The routing field is separated into 2 parts:

i) The UNIX timestamp. Given the application type (defined in (iii) of Header), the timestamp can represent the current time, or the deadline (TTL) of the advertised message, or the deadline of the OB service.

ii) The current hop information of the message (hops-to-go). The given hop limit is decremented by one in each message hop. When the hop count reaches to zero, the message is dropped and not taken into account in the network anymore.

In SSID encoding, 5 ASCII bytes are required to encode a UNIX timestamp with Base 94 conversion. To define hops-to-go, 1 ASCII byte is required. Thus, the maximum hop limit can start from 94 (The same encodings hold for Bluetooth network name). For UUID, 32 bits are required to encode a UNIX timestamp whereas 8 bits are required to encode hops-to-go.

\section{QoS}

The QoS field carries $I_{Q o S}$ of an application, i.e. the dynamic service characteristics that are advertised in the network. By reading $I_{Q o S}$ of an OB, the BOs in proximity can decide on what kind of application or service they can participate. As further given in Section 3.4, $I_{Q o S}$ is composed of 4 different dynamically-updated metric values. Accordingly, each representing a metric value, the QoS field consists of 4 parts:

i) Contact stability (CS) value, to show how stable the contacts are over time.

ii) Contact diversity $(\mathrm{CD})$ value, to show how diverse the contacts are over time.

iii) Data stability (DS) value, to show how stable the data is over time.

iv) Data diversity (DD) value, to show how diverse the data is over time.

In SSID orientation, each of the parts in the QoS field is encoded with 1 ASCII byte (Same holds for Bluetooth network name). In UUID, 8 bits are used for each. Thus, there might be 94 different levels to represent the values of the QoS metrics in the SSID encoding whereas 256 levels in the UUID encoding.

\section{Payload}

The remainder bytes / bits can be used for payload. The payload can contain $I_{A}$ or user-generated information. It can also be utilized to encode additional $I_{R}$ or $I_{Q o S}$.

In SSID encoding, the payload can be up to 20 bytes. On the other hand, UUID encoding can only hold 20 bits of payload to represent simple flags or low-precision data. 


\subsubsection{Applicability Study}

In order to test the performance of opportunistic beacons, Wi-Fi and BLE are investigated. The applicability tests are taken with Samsung S2 and S4 Mini smartphones on Android v4.4 and Motorola Moto G smartphones and Nexus 7 tablets on Android v5.0.

\section{Wireless Operations}

Several wireless operations related to the utilization of opportunistic beacons are evaluated in terms of their time of operation and power consumption. The operations are as follows: beaconing, scanning, enabling/disabling the beaconing and scanning modes.

To actualize the concepts of $\mathrm{OB}$ and $\mathrm{BO}$ with the IEEE 802.11 standard, Wi-Fi Hotspot and Wi-Fi Infrastructure modes are utilized, respectively. The OB and BO roles are also implemented with the use of BLE Peripheral mode and BLE Central mode, respectively.

Beacon and scan intervals: For Wi-Fi, beacon interval $\left(t_{B I}\right)$ is standardized as $100 \mathrm{~ms}$ by default. For scanning times $\left(t_{S I}\right)$, on the other hand, Wi-Fi reflects non-deterministic values based on adapter capabilities. Complying the standards, mobile O/S platforms perform network scans including all WLAN channels and this is non-adjustable. According to the IEEE 802.11 specification [123], there are two scan types: passive and active. Passive scanning cannot be deactivated, and its $t_{S I}$ depends on environmental factors such as interference. Nevertheless, BOs utilize active scanning which is more faster. For a single WLAN channel, the representative active scan period is $10 \mathrm{~ms}$ by default. For all 13 WLAN channels, $t_{S I}$ is expected to be $130 \mathrm{~ms}$ at minimum.

As a simpler interface, BLE introduces 3 advertisement channels on the $2 \mathrm{MHz}$ band. As a result, the scan procedure of BLE takes considerably less time than of IEEE 802.11. According to the BLE specification [124], $t_{S I}$ ranges between $0.6 \mathrm{~ms}$ and $1.2 \mathrm{~ms}$ in Central mode. On the other hand, $t_{B I}$ can be set in a range of from $20 \mathrm{~ms}$ to $10,000 \mathrm{~ms}$ in Peripheral mode. In only Android and $i O S, t_{B I}$ is programmatically adjustable. The $i O S$ guide in [125] recommends the following $t_{B I}$ values to boost chances of advertisement beacon discovery (ms): 645, 768, 961, 1065, 1294. By the Android developer team, no recommendation is given for this. Under any circumstances, these $t_{S I}$ and $t_{B I}$ values are pretty promising for OppNet development with opportunistic beacons.

Table 3.1 shows the standard deviation values $(\sigma)$ of $815 t_{B I}$ and $t_{S I}$ measurements obtained with 5 Motorola Moto $G$ smartphones. In conformity with its standard value, $t_{B I}$ is measured as $\approx 100 \mathrm{~ms}$ for Wi-Fi Hotspot mode. Tested with $100 \mathrm{~ms}$ on Android, the obtained $t_{B I}$ values for BLE Peripheral mode are quite consistent as well. On the other hand, $t_{S I}$ is non-deterministic. Wi-Fi Infrastructure mode reflects varying $t_{S I}$ values. In our tests, $t_{S I}$ is set as $3000 \mathrm{~ms}$ for both Wi-Fi Infrastructure and BLE Central modes. The obtained $t_{S I}$ results show a slight variation for both interfaces.

TABLE 3.1: Beacon and Scan Operations

\begin{tabular}{llll}
\hline Operation & Time Interval & Wi-Fi & BLE \\
\hline Beacon & $t_{B I}$ & $\sigma=0.014 m s$ & $\sigma=0.022 m s$ \\
\hline Scan & $t_{S I}$ & $\sigma=247 m s$ & $\sigma=12 m s$ \\
& & & 3 CocOON ArCHITECTURE
\end{tabular}


Switch durations: Figure 3.6 shows the cumulative distribution function of the execution times measured with $\approx 52,000$ unique Wi-Fi Infrastructure and Wi-Fi Hotspot enable/disable operations which run during OB-BO transitions. It is evident that the execution times show definite variations majority of the time. However, some outlier measurements are present due to the $\mathrm{O} / \mathrm{S}$ platform limitations.

For BLE, switching between Peripheral mode and Central mode does not require to enable/disable the Bluetooth adapter, and is a matter of activating these modes programmatically. The execution time required for activation of these modes is very short, at milliseconds level.

Power consumption: Table 3.2 shows the average battery percentage usage ( $\mu \mathrm{BPU}$ ) results obtained for different configurations of our automaton. Each configuration testing is carried out for $\approx 6$ hours on a Motorola Moto G smartphone. For both Wi-Fi and $\mathrm{BLE}, t_{B I}$ is set to $100 \mathrm{~ms}$ and $t_{S I}$ is set to $3000 \mathrm{~ms}$. Unquestionably, the absolute BPU results can differ on different hardware platforms. Nevertheless, the obtained $\mu$ BPU results for different configurations relate with each other. In comparison to the dedicated ad hoc interfaces, several OB-BO arrangements demand considerably low energy. For instance, $\mu \mathrm{BPU}$ of $\mathrm{Wi}-\mathrm{Fi}$ Ad-Hoc mode is measured as $18.32 \% / \mathrm{h}$ on a Nexus 7 device. This is almost 3 times higher BPU than of our $t_{O B}=t_{B O}=15 \mathrm{~s}$ configuration set with Wi-Fi. For OBN scenarios, this simply implies an increased energy efficiency compared to conventional ad hoc networking protocols. For OAN scenarios, $\mu \mathrm{BPU}$ is highly dependent on several in-network factors such as different OB-BO arrangements and data

FigURE 3.6

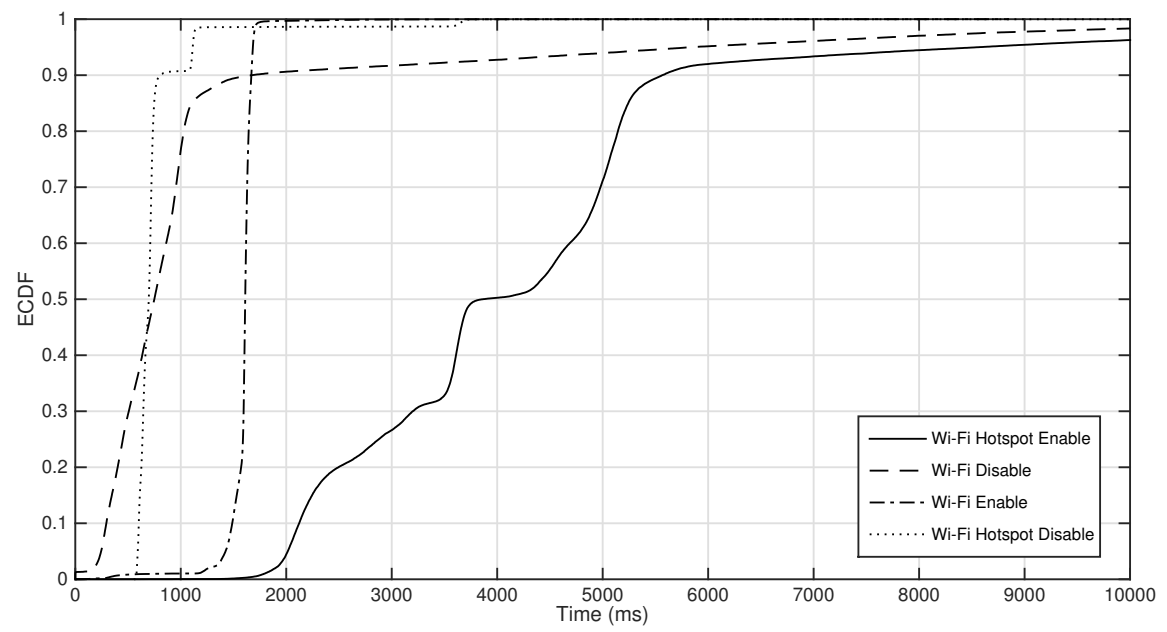

Execution time measurements of Wi-Fi operations: Including results obtained with 1 Samsung S2, 5 Samsung S4 Mini, 5 Motorola Moto G phones and 4 Nexus 7 tablets. 
TABLE 3.2: $\mu$ BPU measurements

\begin{tabular}{llll}
\hline Mode & Service Time & $\mathbf{8 0 2 . 1 1}$ & BLE \\
\hline Idle & - & $0,61 \% / \mathrm{h}$ & $0,61 \% / \mathrm{h}$ \\
\hline $\mathrm{BO} \circlearrowleft$ & $t_{B O}=\infty$ & $0,76 \% / \mathrm{h}$ & $0,81 \% / \mathrm{h}$ \\
\hline $\mathrm{OB} \leftrightarrows \mathrm{BO}$ & $t_{O B}=t_{B O}=45 \mathrm{~s}$ & $3,82 \% / \mathrm{h}$ & $2,03 \% / \mathrm{h}$ \\
\hline $\mathrm{OB} \leftrightarrows \mathrm{BO}$ & $t_{O B}=t_{B O}=30 \mathrm{~s}$ & $3,87 \% / \mathrm{h}$ & $2,11 \% / \mathrm{h}$ \\
\hline $\mathrm{OB} \leftrightarrows \mathrm{BO}$ & $t_{O B}=t_{B O}=15 \mathrm{~s}$ & $3,94 \% / \mathrm{h}$ & $2,15 \% / \mathrm{h}$ \\
\hline $\mathrm{OB} \circlearrowleft$ & $t_{O B}=\infty$ & $4,17 \% / \mathrm{h}$ & $2,82 \% / \mathrm{h}$ \\
\hline
\end{tabular}

transmissions occurring through these arrangements. Nevertheless, our OAN protocol provides more energy efficiency than Wi-Fi Ad Hoc mode since not all of the network devices need to perform the costly beaconing operation. In [41], a similar conclusion is already pointed out for WiFi-Opp which is an OAN-like protocol.

\section{Nodal Density}

A contention problem can arise when several devices simultaneously relay scan probe requests and/or beacon advertisements/responses. In dense networks, beacon collisions dramatically lower the neighbor discovery rates due to high number of probe requests/responses [126]. In order to avoid this, Wi-Fi and Bluetooth employ their own MAC clock synchronization protocols. IEEE 802.11 uses Carrier Sense Multiple Access with collision avoidance (CSMA/CA) whereas Bluetooth (including BLE) presents Clock Synchronization Protocol (CSP) under its multi-channel adaptation protocol. CSMA/CA and CSP utilize a special timing parameter called contention window to reduce the number of packet collisions. In case the shared channel is busy, each device backs off for a random period by increasing its contention window exponentially. Once the channel becomes idle, contention window is set to its minimum value. This is handled at nanosecond level for both $\mathrm{Wi}-\mathrm{Fi}$ and BLE, and significantly reduces the number of beacon collisions [126]. According to the IEEE 802.11 specification (Section 9.4.2) and Bluetooth $\mathrm{v} 4.2$ specification (Section 8.4 .3 ), the contention-free period is $\approx 250 \mathrm{~ms}$ in the worst case.

These officially specified validations clearly indicate that the system may not be collisionfree; there is a probability that at least two contention windows can expire at the same time in a network. However, this possibility is less likely to occur since contention window can grow up to 1023. This constitutes an advantage the OBN data exchange protocol even under high device density. However, it is worth to note that high device density may cause connectivity and data transmission issues in OAN scenarios.

\section{Mobility}

To test beacon reception rates in highly-mobile environments, a mobility evaluation is carried out as well. Under several inter-device relative velocities, the $\mathrm{OB}$ reception performance is assessed with an experimental Wi-Fi Hotspot/Infrastructure setup. The setup consists of two mobility groups separated into two vehicles, each including 5 Samsung S4 Mini smartphones. 
In opposite directions along a directional road, the vehicles have shuttled between two base points each time with a different constant velocity. Before each departure from a base point, all phones have created a unique SSID. At the halfway point, packet switching has been tested each time for the following OBN scenarios:

I) Groups 1 and 2 have run as OBs and BOs, respectively.

II) Groups 1 and 2 have run the presented state diagram to enable OB-BO switching, initiated at random times, with $t_{O B}=t_{B O}=5 \mathrm{~s}, 15 \mathrm{~s}, 30 \mathrm{~s}$.

Figure 3.7 shows the average packet reception rates (PRRs) of (I) and (II) with regard to different relative velocities. Since (I) is one-directional packet forwarding, its PRR is $50 \%$ at most, decreases down to $41 \%$ when the relative velocity is $33 \mathrm{~m} / \mathrm{s}(120 \mathrm{~km} / \mathrm{h})$.

Figure 3.7

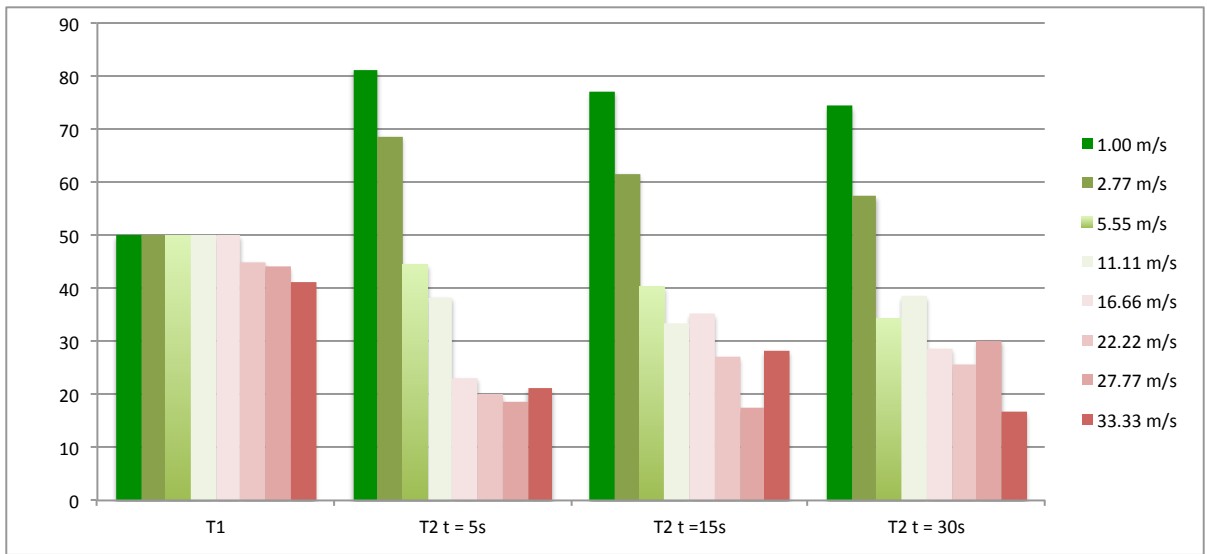

Mobility Test Results: Packet reception rates are shown with respect to different tests

On the contrary, the PRR of (II) is higher than of (I) at low velocities, however, significantly decreases down to $18 \%$. The reason is twofold: First, increasing relative velocity decreases contact durations. During a short contact period, it is less probable to complete a single OB-BO cycle, i.e. a node cannot have enough time to serve as an $\mathrm{OB}$ and subsequently as an $\mathrm{BO}$, or vice versa, over the course of its contact period with another node in radio reach. Second, the operations of the nodes in contact can overlap, i.e. both can serve as an OB or as an $\mathrm{BO}$ at the same time. 


\subsection{QOS ModeL}

The Cocoon architecture employs a QoS model for the quantitative and dynamic evaluation of the above-presented criteria in the application scheduling and networking selection processes. The QoS model utilizes a derived form of Analytic Network Process (ANP) introduced by Saaty [127]. ANP is a practical decision-making tool developed for resolving the complexity of multi-criteria evaluation processes. ANP is widely acclaimed by researchers of various fields for more than a decade. ANP incorporates inter-relationships of factors that have influence on a specific objective.

The QoS model objective is to select the most appropriate application for current operation according to the present state of network conditions. Unquestionably, these conditions may vary over time. Thus, the model must have a feedback routine that collects information about the current network characteristics. By default, the inter-relationships between a set of influential factors are predefined in the standard ANP design. Our QoS model, nevertheless, uses dynamic inter-dependency and feedback routines between the defined factors and the candidate applications for operation, allowing inclusion of periodically-updated information in the decision-making process.

\subsubsection{QoS REQUIREMENTS}

In OppNets, the number and diversity of contacts varies from time to time. The networking goal of an OppNet application can be either data dissemination, or data routing, or both. For dissemination-oriented goals, diversity in encountered contacts gains importance. Thus, data scalability can increase. For routing-oriented goals, on the contrary, stability in number of encountered contacts gains importance. Thus, more reliable data switching can be performed. Similarly, the amount and diversity of data in OppNets varies from time to time. In terms of data utilization, the goal of an OppNet application can be based on different data collection or data sharing strategies. Some applications might require high diversity of data, some might be interested in one type of data, etc.

In consideration of these facts, the requirements for our service management are given in Table 3.3, each defined as a separate criterion for fulfilling a corresponding network objective. All of the below-referred expressions and formulas form the definition set of the QoS-specific data of a device, i.e. $I_{Q o S}$.

TABLE 3.3: Service Requirements

\begin{tabular}{ll}
\hline Criterion & Definition \\
\hline Contact Stability (CS) & $\begin{array}{l}\text { An absence of excessive fluctuations in the same group of } \\
\text { contacts encountered within a specified time period. }\end{array}$ \\
\hline Contact Diversity (CD) & $\begin{array}{l}\text { A presence of excessive fluctuations in the number } \\
\text { contacts encountered within a specified time period. }\end{array}$ \\
\hline Data Stability (DS) & $\begin{array}{l}\text { An absence of excessive fluctuations in the same type of } \\
\text { data collected within a specified time period. }\end{array}$ \\
\hline Data Diversity (DD) & $\begin{array}{l}\text { A presence of excessive fluctuations in the number } \\
\text { different data types collected within a specified period. }\end{array}$ \\
\hline
\end{tabular}




\subsubsection{QOS CHARACTERIZATION}

Following the ANP methodology, our QoS model generation is composed of the following major steps:

\section{Model Construction}

As the initial step, a decision network has to be formulated. Our objective of selecting the most appropriate application for operation is decomposed into a rational system, a decision network that comprises formal definitions for the inter-relations between defined criteria. The QoS management in each device is dynamically characterized with the total of the networking capabilities, per application capabilities, per application requirements, and per application priority of the device. As shown in Figure 3.8, the structure of the decision network is composed of the above-defined criteria, alternatives (i.e. applications), and their inter- and intra-relationships. These relationships constitute our four decision-making clusters: i) Criteria Interdependence (CI), ii) Application Interdependence (AI), iii) Network Feedback (NF), and iv) Priority Feedback (PF). Either dynamically or statically, all clusters are set a reciprocal value for each of their pair-to-pair relations. A pairwise comparison is the following function is demonstrated as $r_{C}(x, y)$, where $x$ and $y$ are element of a decision cluster $C$.

AI holds the static relationship information between each application running in the local OppNet of a device. That is, the predefined requirements of applications are defined in terms of a level of requirement (i.e. predefined importance) for each of the criterion, thus these levels are reflected to AI. Representing its type, an application $\alpha_{i}$ is defined and registered in the architecture with predefined requirement levels for each criterion. The requirement levels of $\mathrm{CS}, \mathrm{CD}, \mathrm{DS}$, and DD for $\alpha_{i}$ are respectively denoted as $r_{A I}\left(C S, \alpha_{i}\right), r_{A I}\left(C D, \alpha_{i}\right), r_{A I}\left(D S, \alpha_{i}\right)$, and $r_{A I}\left(D D, \alpha_{i}\right) \in \mathbb{Z}[1-9]$. A requirement level simply tells how important a criterion for $\alpha_{i}$, ranging from 1 (the least important) to 9 (the most important).

PI holds the dynamically updated priority relations of the applications running in the local OppNet. the dynamic priority level of $\alpha_{i}$ is expressed as $P\left(\alpha_{i}\right) \in \mathbb{Z}[1-9]$.

NF holds the dynamically updated relationship of the networking capabilities of the applications running in the local OppNet in comparison to other applications. Representing its present networking capability, in each automaton cycle, $\alpha_{i}$ is assigned an updated capability

Figure 3.8

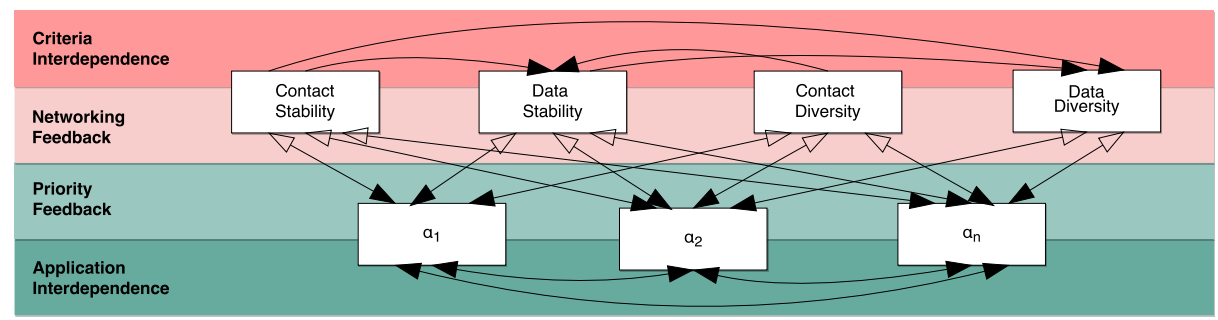

The ANP model 
level for each criterion. The actual capability levels for CS, CD, DS, and DD are respectively denoted as $L_{C S}\left(\alpha_{i}\right), L_{C D}\left(\alpha_{i}\right), L_{D S}\left(\alpha_{i}\right)$, and $L_{D D}\left(\alpha_{i}\right)$.

$\mathrm{CI}$ holds the dynamic relationship information between each criterion. That is, the importance of a criterion can increase or decrease as the mobile network characteristics change. These changes are observed locally and reflected to CI. Representing its present networking capabilities, a device $d_{i}$ is assigned dynamic importance levels for each criterion. The actual importance levels for CS, CD, DS, and DD are respectively denoted as $L_{C S}\left(d_{i}\right), L_{C D}\left(d_{i}\right)$, $L_{D S}\left(d_{i}\right)$, and $L_{D D}\left(d_{i}\right)$.

For the determination of individual $L_{C D}\left(\alpha_{i}\right)$ and $L_{D D}\left(\alpha_{i}\right)$, Shannon's well-accepted normalized Entropy Index is utilized. The normalized entropy, denoted as $H_{n}(X)$, quantifies the diversity present in the distribution of a set $X$. It is defined as,

$$
H_{n}(X)=\frac{-\sum_{x \in X} p(x) \log p(x)}{\log |X|}
$$

where $x$ denotes a value that $X$ can adopt from the set $X$. To compute this, the value (or an estimate) of the distribution $p(x)$ must be known. When $X$ is discrete, this can be measured by frequency counts from data, that is $p(x)=\frac{|x|}{|X|}$, the fraction of observations taking on value $x$ out of the total number of elements in $X$. If all events are equally likely, that is, maximum uncertainty over the outcome, then $H_{n}(X)$ is maximum, i.e. 1. If the distribution is highly biased toward one particular event $x \in X$, that is, little uncertainty over the outcome, then the normalized entropy is low.

To compute Shannon's Entropy Index for $L_{C D}\left(\alpha_{i}\right),|x|$ is set as the number of encountered contacts by $\alpha_{i}$ within the previous automaton cycle (that is earlier denoted as $T$ in Section 3.2.1). Similarly, $|X|$ is set as the number of all encountered contacts by $d_{i}$ within $T$. Similarly, for $L_{C D}\left(\alpha_{i}\right),|x|$ is set as the number of received/generated data packets by $\alpha_{i}$ within $T$ in Section 3.2.1) whereas $|X|$ is set as the number of all received/generated data packets by $d_{i}$ within $T$. Additionally to compute stability, first we provide Mutual Information introduced by Shannon, between $X$ and $Y$, that is, the amount of information shared by $X$ and $Y$, is formulated as follows:

$$
I(X, Y)=\sum_{x \in X} \sum_{y \in Y} p(x, y) \log \frac{p(x, y)}{p(x) p(y)}
$$

Stability Index, namely Consistency Index as its original name introduced by Yu et al. [128], is used to measure $L_{C S}\left(\alpha_{i}\right)$ and $L_{D S}\left(\alpha_{i}\right)$ of any $\alpha_{i}$. Between two instances of a distribution set $X$ occurring at different times, $t_{1}$ and $t_{2}$, Stability Index is formulated as follows:

$$
w\left(t_{1}, t_{2}\right)=\frac{I\left(X_{t_{1}}, X_{t_{2}}\right)}{H\left(X_{t_{1}}\right)+H\left(X_{t_{2}}\right)}
$$

Stability Index takes values in range [0,0.5], with a zero value indicating a strong anti-correlation between the features sets, a positive value indicating similar sets. Note that Stability Index does not tell us anything about the relationships between the variables and the outcome, but merely that the distribution of an element $x$ in the distribution set $X$ has changed. 
For a specific $T$ period, $L_{C S}\left(d_{i}\right), L_{D S}\left(d_{i}\right), L_{C D}\left(d_{i}\right)$, and $L_{D D}\left(d_{i}\right)$ are the mean values of the sum of all their corresponding $L_{C S}\left(\alpha_{i}\right), L_{D S}\left(\alpha_{i}\right), L_{C D}\left(\alpha_{i}\right)$, and $L_{D D}\left(\alpha_{i}\right)$, respectively.

\section{Pairwise Comparisons}

For the determination of a $r(x, y)$ in the clusters $\mathrm{AI}, \mathrm{CI}$, and PF, the quotient between $x$ and $y$ is utilized, i.e. $r(x, y)=x / y$. For NF, $r(x, y)$ is $L_{x}(y)$ where $x$ denotes a criterion and $y$ denotes an application. For each cluster, the relative pairwise comparison matrix is denoted as $A_{C}$, where $C$ denotes the cluster. The generated matrices for relative pairwise comparisons are given in Equations 3.5, 3.6, 3.7, and 3.8 for the clusters AI, CI, PF, and NF, respectively.

$A_{A I}, A_{C I}, A_{P F}$, and $A_{N F}$ are unweighted. For the following ANP processes, they are normalized and converted to the weighted matrices of $W_{A I}, W_{C I}, W_{P F}$, and $W_{N F}$ with the generic normalization equation given in Equation 3.4 where $w$ is the eigenvector and $\lambda_{\max }$ is the largest eigenvalue of $A_{C}$ :

$$
\begin{aligned}
& A_{C} \times w=\lambda_{\max } \times w \\
& A_{A I}=\begin{array}{c}
\alpha_{1} \\
\mathrm{DS} \\
\mathrm{CD}
\end{array}\left[\begin{array}{ccc}
\mathrm{r}_{A I}\left(C S, \alpha_{1}\right) & \ldots & \mathrm{r}_{k} \\
\mathrm{r}_{A I}\left(D S, \alpha_{1}\right) & \ldots & \mathrm{r}_{A I}\left(D S, \alpha_{k}\right) \\
\mathrm{r}_{A I}\left(C D, \alpha_{1}\right) & \ldots & \mathrm{r}_{A I}\left(C D, \alpha_{k}\right) \\
r_{A I}\left(D D, \alpha_{1}\right) & \ldots & \mathrm{r}_{A I}\left(D D, \alpha_{k}\right)
\end{array}\right] \\
& A_{C I}=\begin{array}{c}
C S \\
C S \\
\mathrm{DS} \\
\mathrm{CD}
\end{array}\left[\begin{array}{llc}
r\left(L_{C S}\left(d_{i}\right), L_{C S}\left(d_{i}\right)\right) & \ldots & r\left(L_{C S}\left(d_{i}\right), L_{D D}\left(d_{i}\right)\right) \\
\mathrm{DD}\left(L_{D S}\left(d_{i}\right), L_{C S}\left(d_{i}\right)\right) & \ldots & r\left(L_{D S}\left(d_{i}\right), L_{D D}\left(d_{i}\right)\right) \\
r\left(L_{C D}\left(d_{i}\right), L_{C S}\left(d_{i}\right)\right) & \ldots & r\left(L_{C D}\left(d_{i}\right), L_{D D}\left(d_{i}\right)\right) \\
r\left(L_{D D}\left(d_{i}\right), L_{C S}\left(d_{i}\right)\right) & \ldots & r\left(L_{D D}\left(d_{i}\right), L_{D D}\left(d_{i}\right)\right)
\end{array}\right]
\end{aligned}
$$

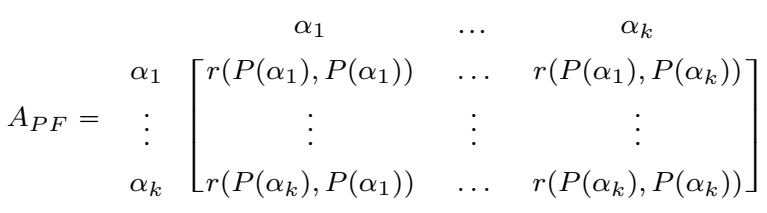

$$
\begin{aligned}
& \begin{array}{c}
\multicolumn{1}{c}{C S} \\
A_{N F}= \\
\alpha_{1} \\
\vdots \\
\alpha_{k}
\end{array}\left[\begin{array}{cccc}
L_{C S}\left(\alpha_{1}\right) & L_{D S}\left(\alpha_{1}\right) & L_{C D}\left(\alpha_{1}\right) & L_{D D}\left(\alpha_{1}\right) \\
\vdots & \vdots & \vdots & \vdots \\
L_{C S}\left(\alpha_{k}\right) & L_{D S}\left(\alpha_{k}\right) & L_{C D}\left(\alpha_{k}\right) & L_{D D}\left(\alpha_{k}\right)
\end{array}\right]
\end{aligned}
$$




\section{Supermatrix Generation}

The supermatrix from the overall weights of the clusters is generated with the orientation given in Equation 3.9. The upper part of the supermatrix holds the interdependece values of our decision network. On the other hand, the lower part of the supermatrix holds the feedback values of our decision network.

The supermatrix concept is similar to the Markov chain process [129]. To obtain global weighted importance values in the decision network with interdependent influences and feedbacks, the local priority vectors are entered in the appropriate columns of the supermatrix. As a result, the supermatrix is actually a partitioned matrix, where each matrix segment represents a cluster relationship. The final priorities of all elements in the supermatrix can be obtained by normalizing the supermatrix.

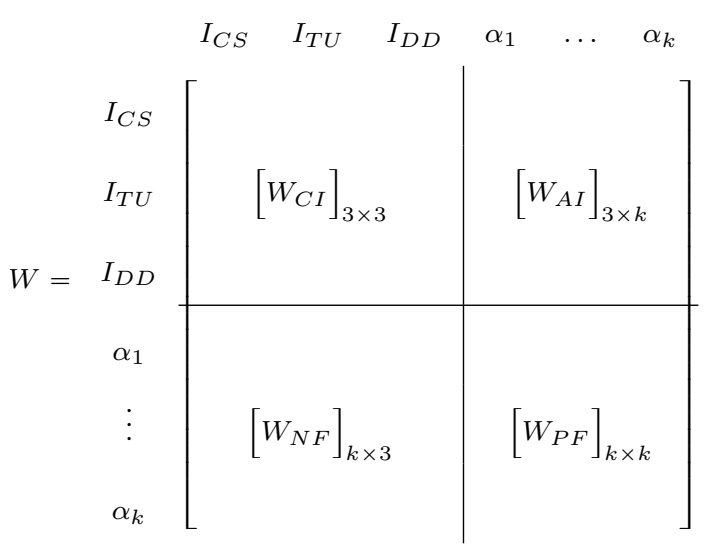

\section{Limit Supermatrix Generation}

Calculating the long-term (stable) weights of the alternatives (applications in our case) is obtained by raising the supermatrix to exponential powers. To achieve convergence on the importance weights, the weighted supermatrix is raised to the power of $2 k+1$, where $k$ is an arbitrarily large number; the newly-generated matrix is called the limit supermatrix as shown in Equation 3.10. The limit supermatrix has the same form as the weighted supermatrix, but all the columns of the limit supermatrix are the same.

$$
W^{*}=\lim _{k \rightarrow \infty} W^{2 k+1}
$$

\section{Evaluating Results}

The alternative (application) with the largest overall priority should be selected, as it is the best alternative as determined by the calculations made using matrix operations. 


\subsection{Concluding Remarks}

This chapter has presented Cocoon, our community-oriented context-aware OppNet architecture. Cocoon is a universal system that is capable of going off-the-grid and enabling rapid ad hoc communication without relying on the traditional connection-based approaches. The connectivity model makes use of smart beacon advertisements so as to provide networking and QoS between devices without connection establishment. Optionally, a connectionbased networking model is presented as well. The presented system is generic in terms of usability on the current mobile O/S systems. As leveraging the devices people use everyday, it supports the development of the OppNet applications intended for any group of users in the general public.

The connectivity scheme of the Cocoon architecture, namely opportunistic beacons, can be implemented above the standard Wi-Fi and Bluetooth interfaces without requiring any configuration or kernel/driver modification. Presented on top of the opportunistic beacons, the networking models of the Cocoon architecture provides a self-configuring and self-organized OppNet groups. Based on the context utilization in a network, the devices can set up provisional networks on a consensus-based decision making. Moreover, they can decide on which application (or message) to support by considering the requirements of running applications in their local network as well as by sensing their network characteristics. 


\section{Opportunistic Beacon Networking}

This chapter presents OpPortunistic Beacon Networking (OBN), that IS ONE OF TWO CORE NETWORKING MODELS INTRODUCED IN THE COCOON ARCHITECTURE. OBN IS A UNIVERSAL OPPORTUNISTIC AD HOC NETWORKING MODEL PARTICULARLY INTENDED FOR SMART MOBILE DEVICES. IT ENABLES FAST AND LIGHTWEIGHT DATA DISSEMINATION IN WIRELESS COMMUNITY NETWORKS THROUGH THE USE OF OPPORTUNISTIC BEACONS PRESENTED IN CHAPTER 3.

OBN IS BASED ON AN AUTOMATIC DATA EXCHANGE PROTOCOL THAT CAN OPERATE ON TOP OF ANY WIRELESS INTERFACE SUCH AS WI-FI AND BLUETOOTH. With ITS EASE OF APPLICABILITY, THE PROTOCOL CAN DIRECTLY OPERATE ON ANY SMART MOBILE PLATFORM WITHOUT REQUIRING MODIFICATIONS ON THEIR AFFILIATED WIRELESS ADAPTERS. FroM FEASIBILITY EXPERIMENTS TO SMALLSCALE NETWORKING EXPERIMENTS TO LARGE-SCALE EXPERIMENTS, THE PERFORMANCE OF THE PROTOCOL IS COMPREHENSIVELY EVALUATED WITH BOTH REALWORLD EXPERIMENTS AND VERIFIED SIMULATIONS. FOR AN IMPROVED NETWORKING PERFORMANCE, SEVERAL ENHANCEMENTS ARE OFFERED IN THE PROTOCOL DESIGN. THE PERFORMANCE OUtCOMES CLEARLy INDICATE that the OBN MODEL BRINGS HIGH AD HOC NETWORKING FLEXIBILITY AND SCALABILITY AS WELL AS PROVIDES REASONABLE DATA ROUTING AND DISSEMINATION PERFORMANCE FOR NUMEROUS DECENTRALIZED SOCIAL NETWORKING PURPOSES. TO THIS RESPECT, THE OBN MODEL CAN BE READILY INTEGRATED TO NUMEROUS MOBILE PARTICIPATORY SENSING APPLICATIONS WITHOUT REQUIRING ANY SPECIALIZED SETUP. 


\subsection{Overview}

OBN is a mobile ad hoc networking model which expedites opportunistic information dissemination in communities comprising smart mobile device users. It is a lightweight and platform-independent approach that offers high scalability and an ease of integration to the mobile participatory sensing applications. It involves a delay-tolerant data exchange protocol that can run above any wireless interface supporting beaconing. The protocol can be readily used on top of any Wi-Fi or Bluetooth interface. Employing store-carry-forward fashion, it exploits the concepts of opportunistic beacon (OB) and beacon observer (BO) presented in Chapter 3. As a brief reminder, an $\mathrm{OB}$ is a wireless network identifier field encoded as a specific information, and a device that relays that information is also called as OB. BO, on the other hand, is a device scanning for OBs within its wireless range.

\subsubsection{NeTWORK MODEL}

OBN is illustrated over a network setup in Figure 4.1. In OBN, devices operate in two modes: i) $\mathrm{OB}$ and ii) $\mathrm{BO}$. As previously mentioned, from an $\mathrm{OB}$ to a $\mathrm{BO}$, a packet transmission is one-directional. The reason for this case is that today's wireless adapters cannot concurrently handle beaconing and scanning. Nonetheless, devices exploit wireless broadcast advantage.

FIGURE 4.1

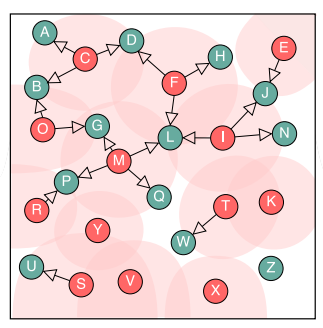

(a) $t_{1}$

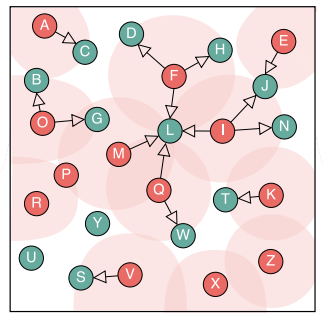

(c) $t_{3}$

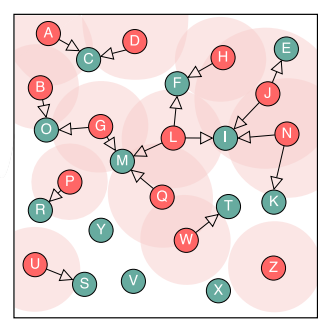

(b) $t_{2}$

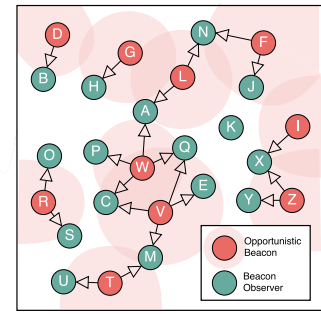

(d) $t_{4}$ 
Multiple OBs can be received by multiple BOs as Figure 1(a) depicts. Devices can periodically switch between the $\mathrm{OB}$ and $\mathrm{BO}$ roles in order to maintain continuous data exchange. In Figure 1(b), an example timely-subsequent to that in Figure 1(a) is shown where all devices switch modes to support full-duplex but delay-tolerant transmissions. Unfortunately, devices serving in same mode cannot discover each other. For instance, both in Figures 1(a) and 1(b), devices $\mathrm{A}$ and $\mathrm{B}$ miss the opportunity to exchange data with each other. To minimize this possibility, OBN offers using adaptive switch cycle durations. As shown in Figure 1(c), this adaptation helps scanning devices to find previously-missed network packets. Another drawback is that a device can broadcast only one packet per cycle. To handle multi-packet transmissions, a packet queueing mechanism is presented for the model.

In comparison to the traditional ad-hoc networking approaches, OBN is a highlyopportunistic but low-throughput approach. To increase network throughput, it gains advantage from device density and mobility. As Figure 1(d) shows, an increase in the number of unique contacts can form a plethora of messages in the network. Moreover, message aggregation techniques presented for identifier encoding can also improve the overall performance.

\subsection{2 РвотосоLs}

As Figure 4.2 depicts, OBN devices employ the automaton presented in Section 3.2.1.

- OB: beaconing mode in order to advertise a pre-encoded network packet.

- BO: scanning mode in order to scan network packets.

- U\&S: transition and packet update mode between OB and BO.

Each state has specific service durations as Figure 4.3 shows. The durations of $\mathrm{OB}$ and $\mathrm{BO}$ are $t_{O B}$ and $t_{B O}$, respectively, which can be adjusted based on network or application needs. At $\mathrm{BO}, t_{S I}$ is the scan interval, i.e. scan operation repeats in every $t_{S I}$. At $\mathrm{OB}, t_{B I}$ is the beacon interval, i.e. announce operation transmits a packet in every $t_{B I}$. At U\&S, activate and deactivate operations together defines the switching duration. Transition from $\mathrm{OB}$ to $\mathrm{BO}$ has a duration of $t_{X B O}$ whereas transition from $\mathrm{BO}$ to $\mathrm{OB}$ has a duration of $t_{X O B}$.

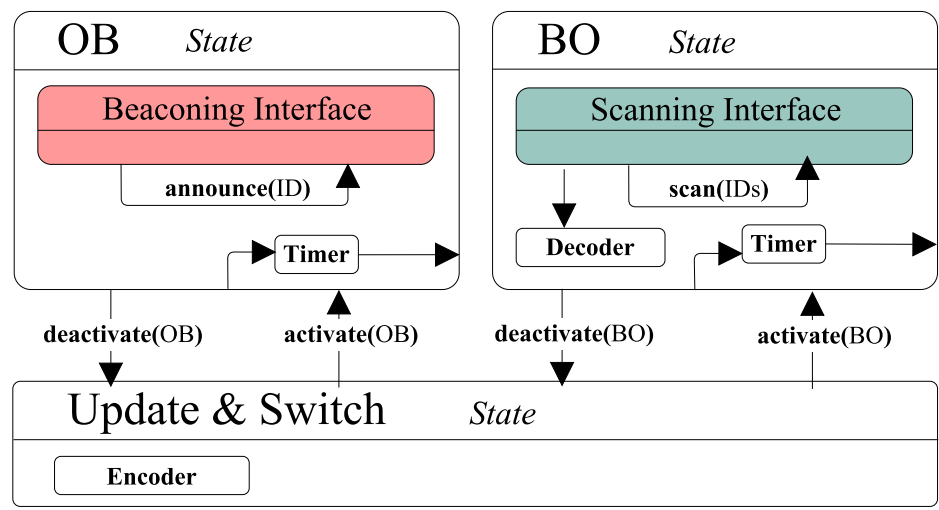

The protocol automaton 


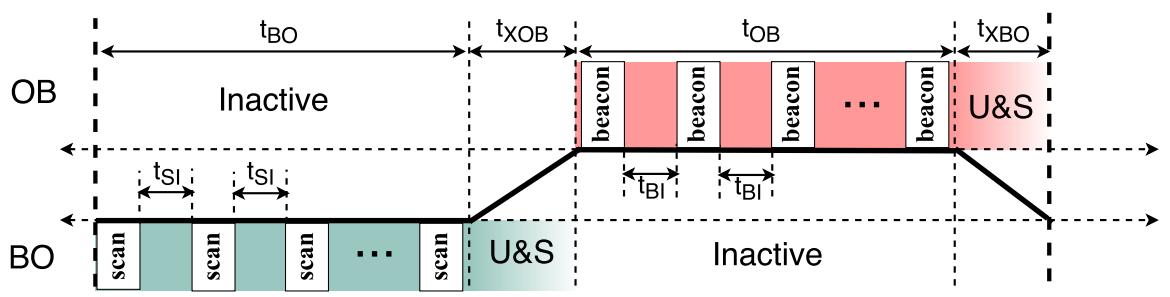

One period of an OB-BO cycle: $t_{B O}+t_{O B}+t_{X O B}+t_{X B O}$

As adapter-specific non-deterministic durations, both $t_{X B O}$ and $t_{X B O}$ help OBN periods to have randomized OB-BO cycles in a network.

To sustain multi-packet transmissions, a circular queue, $Q$, is used. At $\mathrm{U} \& \mathrm{~S}$, the frontmost packet in $Q$ is dequeued prior to an $\mathrm{OB}$ transition. The dequeued packet is encoded in the wireless network identifier. At $\mathrm{OB}$, the encoded packet is broadcast. When OB period is complete, the packet is enqueued back to $Q$. At $\mathrm{BO}$, received (or locally created) packets are enqueued to $Q$. While $Q$ is empty, $\mathrm{OB}$ is not activated, else $\mathrm{BO}$ is repeated. The size of $Q$ can be determined based on network type or application needs. Since large queueing can cause message starvation, the size of $Q$ can be kept fixed, allowing the newest packets to overwrite the oldest ones.

In this chapter, the basic protocol used is based on fixed OB-BO service durations, i.e. $t_{O B}$ and $t_{B O}$ is equal to each other. The non-deterministic $t_{X O B}$ and $t_{X B O}$ allows a randomization in the OB-BO periods. In Section 4.3, the validation experiments are initially tested using this protocol. In Section 4.4, the following enhancements are presented for this protocol.

- Message-adaptive: The OB-BO service times, i.e. $t_{O B}$ and $t_{B O}$, are adjusted based on the number of messages received.

- Time-adaptive: The BO service time of a device, i.e. $t_{B O}$, is adjusted based on the earliest deadline advertised by the OBs in its proximity.

- Dual radio utilization: An additional radio is utilized to complement the OB operation with scanning and the BO operation with advertisement.

The enhancements are tested and compared with additional experimental simulation setups. Over the same setups, all of the presented enhancements are applied at the same time to see their whole effect on the networking performance.

\subsubsection{Chapter Organization}

The rest of the chapter is organized as follows: Section 4.2 first describes the implementations, then additionally gives examples for opportunistic beacon encoding, and finally explains the methodology taken in the tests. Section 4.3 presents the performance analysis. The performance analysis includes validation of the OBN model with small-scale real-world setups and performance outcomes in larger deployments. Section 4.4 introduces the protocol enhancements. The performance improvements are compared with additional experiments. Finally, Section 4.5 gives a brief discussion on OBN. 


\subsection{IMPLEMENTATIONS \& EXPERIMENTS}

This section gives the implementations, testing phases, and the model evaluation parameters. The experiments are taken with both real-world setups and simulations.

For the real-world experiments, an Android application is developed. Using the Cocoon API given in Appendix $C$, the application employs the OBN protocol with the utilization of Wi-Fi Hotspot and Wi-Fi Infrastructure modes in an alternating manner for the OB and BO roles, respectively. As defined in the protocol, Wi-Fi Infrastructure mode constantly runs unless a packet is created or scanned. If a packet is available, SSID is encoded to that packet in advance of beaconing with Wi-Fi Hotspot mode. In each OBN cycle, the application selects the front-most packet of $Q$.

In addition, our event-based simulator, Oppliqué, given in Appendix B is used for additional experiments. The simulator can run the OBN protocol for any kind of network setup and creates network packets as discrete events. At a particular instant in time, each packet is created within a simulated device. Devices mimic the data exchange protocol with specified network parameters. The simulator runs the abstract Wi-Fi PHY/MAC modelling parameters. As previously presented in Section 3.3.3, the wireless operations that have have uncontrollable execution times in reality (such as scanning and enabling/disabling of beaconing mode). The values obtained by the real-world execution of these operations are used in the simulator. Apart from the timely-indefinite operations, the simulator also uses fixed-time Wi-Fi operations. Collected from the Android application runs, the simulator is fed the execution times of real-world values given in Table 4.1.

In OBN, messages can be encoded either with the Cocoon format or independently. Figure 4.4 shows several beacon encoding examples. If not complying with the beacon encoding format presented for the Cocoon architecture in Section 3.3.2, a packet may include more variety of sensor data or related message. An event, a situation, or a condition that has a particular meaning or is applied to a context can be written in place of the reserved fields. Figures 4.4(a) and 4.4(b) deliniate two identifier encoding examples regardless of the Cocoon beacon format, a SSID encoding example and an UUID encoding example. As shown in Figure 4.4(a), encoding may contain additional information such as source identifier, creation time, location, and intermediate router identifiers in case of necessity. As shown in Figure 4.4(b), a packet can also be encoded with multitude of messages. Besides, including a distinctive tag such as a preamble to the packet can be indispensable to distinguish messages inside same network or serving same purposes. Since wireless network identifiers are publicly reachable, encoding may also consider measures against security holes and privacy issues.

TABLE 4.1: Real-world time values used in the simulator

\begin{tabular}{ll}
\hline Symbol & Duration \\
\hline$t_{B I}$ & $\mu=0.100 \mathrm{~s}, \sigma=0.014 \mathrm{~s}$ \\
\hline$t_{X O B}$ & $\mu=4.302 \mathrm{~s}, \sigma=0.524 \mathrm{~s}$ \\
\hline
\end{tabular}

\begin{tabular}{ll}
\hline Symbol & Duration \\
\hline$t_{S I}$ & $\mu=3.000 \mathrm{~s}, \sigma=0.247 \mathrm{~s}$ \\
\hline$t_{X B O}$ & $\mu=3.407 \mathrm{~s}, \sigma=0.327 \mathrm{~s}$ \\
\hline
\end{tabular}

$\mu$ shows the average value, $\sigma$ shows the standard deviation value 


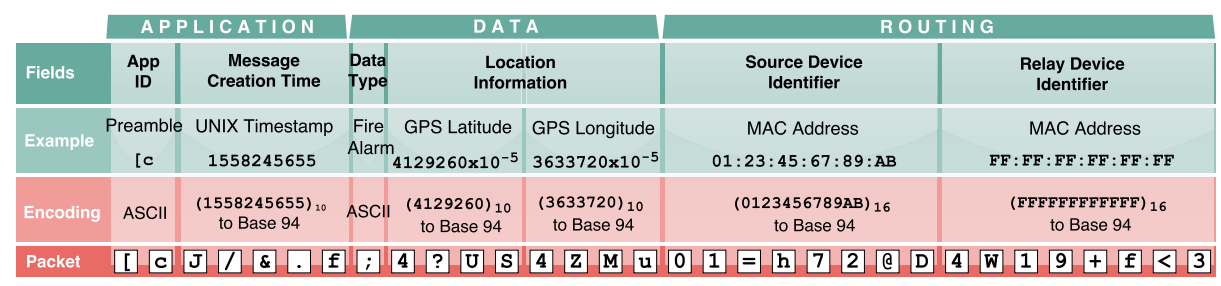

(a) An example for SSID encoding regardless of the Cocoon beacon format

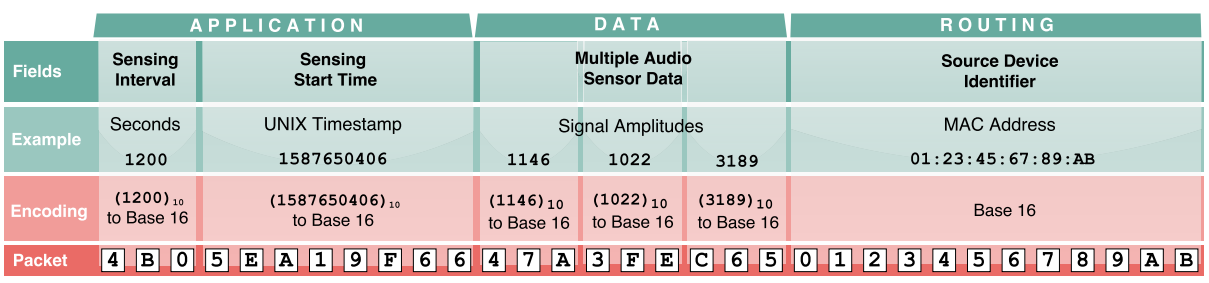

(b) An example for UUID encoding regardless of the Cocoon beacon format

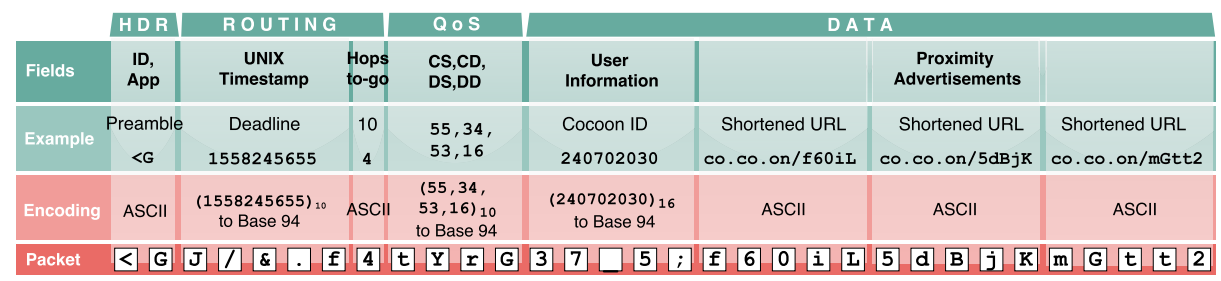

(c) An example for SSID encoding complying with the Cocoon beacon format

Wireless network identifier encoding examples

When complying with the Cocoon beacon format, the message is rather limited in terms of the payload. The payload, which is 20 bytes, is used for application-specific data, that is denoted as $I_{A}$ in Section 3.2.2. Figure 4.4(c) shows a Cocoon beacon example intended for a proximity advertisement use-case. In this example, the payload is divided into two fields. The first field holds the Cocoon user ID while the latter holds three shortened URL data.

For various applications, the payload can be designed differently. In this point, it is also useful to explain how the encoding is applied on the reserved bytes for the Cocoon beacon format (the first 12 bytes). The first field is always used as an identifier which actually holds the application type and priority. In our example, the identifier tells that the beacon is an advertisement message intended for dissemination purpose. That's why the the second field defines a deadline for advertisements. Additionally, the third field holds the current hops-togo value. The last reserved field holds the current QoS information. 


\subsubsection{Testing Phases}

As Table 4.2 shows, the experiments are divided into four testing phases: (1) feasibility and validation tests, (2) parameter tests, (3) small-scale networking tests, and (4) large-scale networking tests. For (1) and (3), both real-world tests and simulations are run with the same evaluation parameters. In real-world experiments, 10 Samsung S4 Mini smartphones are used. The phones run the above-presented Android application. The application creates unique messages with predefined intervals. (2) and (4) are carried out by means of only simulations with varying evaluation parameters and different network setups. In all phases, the tests are taken with each possible combination of the given model parameters. The experimented parameters are explained in Section 4.2.2. The duration of each test is defined as $1800 \mathrm{~s}$.

\section{Validation}

This phase has a twofold aim: (i) to verify the feasibility of our application, and (ii) to validate our simulation modelling. Real-world experiments consist of 10 phones that are situated in range of each other. The simulation is set up in the same manner with the same number of devices. All tests are performed under no mobility. Thus, it is aimed to discover the accuracy of the simulated protocol when mobility effect is discarded.

\section{Parameter Testing}

This phase is performed to find out the effect of each model parameter on the dissemination performance. The tests are carried out by means of simulations and with different number of static devices which are all in range of each other.

\section{Small-scale networking}

The networking model is also assessed over a real-world deployment with 10 participants. Each participant, belonging to an indoor office environment, is given a phone running the presented OBN application. Message switching tests are conducted during office hours along a $37 \times 15 m^{2}$ area with 13 separated rooms.

The same networking environment is simulated with same number of devices and same networking environment. With an extended set of model evaluation parameters, the simulations are run accordingly with the real-world setup. For device movements in the simulations, random waypoint mobility model with pauses is used. The pause time randomly defined for each device ranges between 5 minutes and 30 minutes to mimic working behaviour of the users in the real-world setup.

TABLE 4.2: Experimental Setups

\begin{tabular}{|c|c|c|c|}
\hline \multirow{2}{*}{$\begin{array}{l}\text { Testing } \\
\text { Phase }\end{array}$} & \multirow{2}{*}{$\begin{array}{l}\text { Number } \\
\text { of Devices }\end{array}$} & \multicolumn{2}{|c|}{ Setup Type } \\
\hline & & Real-world & Simulation \\
\hline Validation & 10 & 3 repeated runs & 500 repeated runs \\
\hline Parameter Testing & $5,10,15,20$ & $\mathrm{~N} / \mathrm{A}$ & 500 repeated runs \\
\hline Small-scale Network & 10 & 1 run & 100 repeated runs \\
\hline Large-scale Network & $50,100,150$ & $\mathrm{~N} / \mathrm{A}$ & $\begin{array}{l}100 \text { repeated runs } \\
\text { NISTIC BEACON NETT }\end{array}$ \\
\hline
\end{tabular}




\section{Large-scale networking}

In this phase, the aim is to assess the OBN performance under varying network densities. 3 different network groups are formed: $N_{1}, N_{2}$, and $N_{3}$ consist of 50,100, and 150 devices, respectively. Besides, 5 different network setups are formed with the following networking environment sizes: $M_{1}: 500 \mathrm{~m} \times 500 \mathrm{~m}, M_{2}: 750 \mathrm{~m} \times 750 \mathrm{~m}, M_{3}: 1000 \mathrm{~m} \times 1000 \mathrm{~m}, M_{4}: 1250 \mathrm{~m} \times 1250 \mathrm{~m}$, $M_{5}: 1500 \mathrm{~m} \times 1500 \mathrm{~m}$. The tests are taken for all combinations between the density groups and maps. For device movements in the simulations, random waypoint mobility model with pauses is used. The pause time randomly defined for each device ranges between zero and 3 minutes to mimic behaviour of the users at work in the real-world setup.

\subsubsection{Model Evaluation Parameters}

All tests are conducted with a set of controlled experiments based on varying model evaluation parameters. The repeated test runs in real-world experiments and simulations are conducted for each unique parameter combination given. The experimented design parameters are as follows:

- Service times, $t_{O B}$ and $t_{B O}$, that are assigned to State $\mathrm{OB}$ and State BO, respectively. For all tests, the $\mathrm{OB}$ and $\mathrm{BO}$ service durations are fixed and equal to each other, i.e. $t_{O B}=t_{B O}$. In all phases, the values used for $t_{O B}$ and $t_{B O}$ are $15 \mathrm{~s}, 30 \mathrm{~s}$, and $45 \mathrm{~s}$. The parameter testing phase extends the experiments using intermediate values for $t_{O B}$ and $t_{B O}$ with increments of $5 \mathrm{~s}$.

- Message creation interval, denoted as $t_{M I}$. In a test, a unique event is created in a device at every predefined $t_{M I}$. In the experiments, the values used for $t_{M I}$ range from 30 s up tp 240s, with an increment of 30s.

In brief, Table 4.3 shows the values used in each phase for the given model parameters. The values highlighted in boldface in small-scale networking tests are the ones used in the real-world setup. The simulation runs include tests with all the given parameter values.

TABLE 4.3: Model Evaluation Parameters

\begin{tabular}{lcc}
\hline Testing Phase & \multicolumn{2}{c}{ Model Evaluation Parameters } \\
\cline { 2 - 3 } & $\mathbf{t}_{\mathbf{O B}}=\mathbf{t}_{\mathbf{B O}}(\mathbf{s})$ & $\mathbf{t}_{\mathbf{M I}}(\mathbf{s})$ \\
\hline \multirow{2}{*}{ Validation } & & $30,60,90$, \\
& $15,30,45$ & $120,150,180$, \\
& & 210,240 \\
\hline \multirow{2}{*}{ Parameter Testing } & $5,10,15$, & $30,60,90$, \\
& $20,25,30$, & $120,150,180$, \\
& $35,40,45$ & 210,240 \\
Small-scale Network & $15, \mathbf{3 0}, 45$ & $30, \mathbf{6 0}, 90$, \\
& & $\mathbf{1 2 0}, \mathbf{1 5 0 , \mathbf { 1 8 0 } ,}$ \\
& $15,30,45$ & $210, \mathbf{2 4 0}$ \\
\hline Large-scale Network & & 60,120 \\
\hline
\end{tabular}




\subsubsection{Model Evaluation Metrics}

For brevity and clarity, Table 4.4 lists a set of notations used in the rest of this chapter.

The experimental results presented in Sections 4.3 and 4.4 are evaluated in terms of average dissemination ratio and unidirectional average latency, i.e. with the metrics $D_{M}, D_{\sigma}$, $L_{M}$, and $L_{\sigma}$. In simple terms, the dissemination ratio of a packet defines the number of devices received that packet whereas the unidirectional latency of a packet defines the sum of all latencies divided by the number of devices received that packet.

Additionally in Section 4.4, the end-to-end routing performance of the protocol is experimented as well. The routing performance is evaluated in terms of delivery ratio and number of hops, i.e. with the metrics $R_{M}$ and $H_{M}$.

TABLe 4.4: Notations for Performance Evaluation Metrics

\begin{tabular}{|c|c|}
\hline Symbol & Definition \\
\hline$p_{i}$ & A uniquely created packet in a single test run \\
\hline$P$ & The number of all unique $p_{i}$ 's in a single test run \\
\hline$d_{a}$ & A device in a given setup \\
\hline$N$ & The number of all unique $d_{a}$ 's in a given setup \\
\hline$R_{p_{i}}$ & The number of devices which received $p_{i}$ in a single test run \\
\hline$D_{p_{i}}$ & Dissemination ratio of a unique $p_{i}$, i.e. $R_{p_{i}}$ divided by $N-1$ \\
\hline$D_{\mu}$ & Average of all $D_{p_{i}}$ in a test run, i.e. sum of all $D_{p_{i}}$ divided by $P$ \\
\hline$D_{M}$ & Average of all $D_{\mu}$ for a given setup \\
\hline$D_{\sigma}$ & Standard deviation of all $D_{p_{i}}$ for a given setup \\
\hline$L\left(m_{i}, d_{a}\right)$ & Latency of a unique $p_{i}$ from its source to another device $d_{a}$ \\
\hline$L_{m_{i}}$ & Average latency of all $L\left(p_{i}, d_{a}\right)$ for a unique $p_{i},\left(d_{a} \in R_{p_{i}}\right)$ \\
\hline$L_{\mu}$ & Average of all $L_{p_{i}}$ in a single test run \\
\hline$L_{M}$ & Average of all $L_{\mu}$ for a given setup \\
\hline$L_{\sigma}$ & Standard deviation of $L_{\mu}$ for a given setup \\
\hline$R\left(m_{i}, d_{a}\right)$ & Delivery ratio of a unique $p_{i}$ from its source to destination $d_{a}$ \\
\hline$R_{\mu}$ & Average of all $R\left(m_{i}, d_{a}\right)$ in a test run, i.e. sum of all $R\left(m_{i}, d_{a}\right)$ divided by $P$ \\
\hline$R_{M}$ & Average of all $R_{\mu}$ for a given setup \\
\hline$H\left(m_{i}, d_{a}\right)$ & Number of hops of a unique $p_{i}$ between its source and destination $d_{a}$ \\
\hline$H_{\mu}$ & Average of all $H\left(m_{i}, d_{a}\right)$ in a test, i.e. sum of all $H\left(m_{i}, d_{a}\right)$ divided by $P$ \\
\hline$H_{M}$ & Average of all $H_{\mu}$ for a given setup \\
\hline
\end{tabular}




\subsection{Performance Analysis}

The results of the experiments are presented under four sub-sections:

- Section 4.3.1: Simulation validation test results are given. For both real-world experiments and simulations, the results obtained with the same parameters are compared.

- Section 4.3.2: Parameter testing results are given. In the simulations, the parameters are tested and compared in terms of their effect on the data dissemination performance.

- Section 4.3.3: Small-scale networking evaluation is given. For both real-world experiments and simulations, the results obtained with the same parameters are compared.

- Section 4.3.4: Large-scale networking evaluation is given. For different network setups and OBN model parameters, the experiments are held by means of simulations.

\subsubsection{VALidation}

The real-world experiments and simulations are run 3 times and 500 times, respectively, per unique parameter combination. In all runs, $N=10$. Figure 4.5 shows the results of the simulation validation tests. Under different $t_{M I}$ settings ranging from $30 \mathrm{~s}$ up to $240 \mathrm{~s}$, the real-world test results and the simulation test results are shown together for different $t_{O B}=t_{B O}$ settings.

The real-world tests and simulations are together depicted in Figure 4.5(a) regarding their data dissemination performance. For all network settings, the simulation results are in line with the real-world test results. Nevertheless, minor deviations are notable. The $D_{M}$ values obtained in the simulations are from $\approx 5$ to $\approx 10 \%$ higher than the $D_{\mu}$ values obtained in the real-world setups. Especially for $t_{O B}=t_{B O}=45 \mathrm{~s}$, there are highly-deviated real-world $D_{\mu}$ values lower than their corresponding $D_{M}-D_{\sigma}$ ranges in the simulations. For the same network settings, the difference in average latency between real-world tests and simulations is depicted in Figure 4.5(b). Only two real-world $L_{\mu}$ values are recorded as outliers-one for $t_{O B}=t_{B O}=45 \mathrm{~s}$ and $t_{M I}=180 \mathrm{~s}$ and one for $t_{O B}=t_{B O}=45 \mathrm{~s}$ and $t_{M I}=210 \mathrm{~s}$, which are greater than their corresponding $L_{M}+L_{\sigma}$ ranges in the simulations. These deviations might be related either to the limited number of the physical world tests, or to the inimitable environmental factors affecting the PHY / MAC operations in reality. It is nonetheless evident that the simulation results highly correlate with the corresponding results obtained from real-world experiments.

The simulation validation results also reflect the OBN characteristic when all devices are in range of each other. Under no mobility, the pure effects of $t_{M I}$ and $t_{O B}=t_{B O}$ on the dissemination performance are visible. As expected, a regular increase of the message creation intervals linearly increases the dissemination rates and linearly decreases the latencies. On the other hand, making OBN cycles more frequent has a positive effect on the performance.

\subsubsection{Parameter Testing}

Different $t_{O B}=t_{B O}$ and $t_{M I}$ values are tested in a static network with different number of devices. The aim of this test is to show the effect of each parameter on the OBN performance under no mobility. Figure 4.6 shows the data dissemination performances obtained from the 500 -times repeated simulation runs for all combinations of model evaluation parameters. 


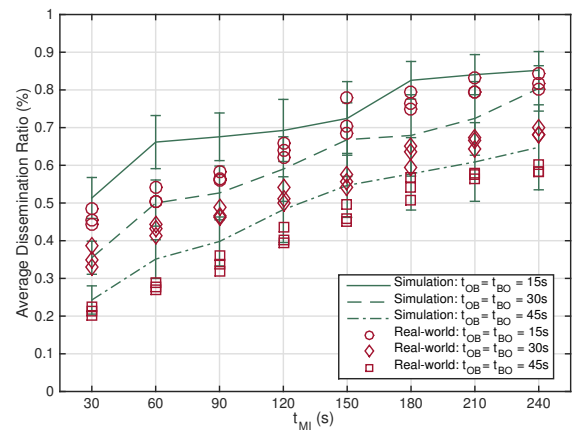

(a)

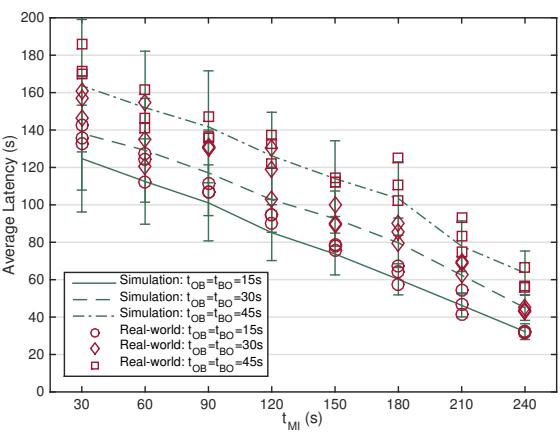

(b)

Simulation Validation Test Results. Corresponding to different $t_{M I}$ settings, the plot lines indicate $D_{M}$ in (a) and $L_{M}$ in (b) whereas the ends of the error bars indicate $D_{\sigma}$ in (a) and $L_{\sigma}$ in (b) of the simulation results. The real-world results are shown as a collection of points (boxes) grouped under different $t_{M I}$ settings. Each point represents a unique $D_{\mu}$ in (a) and $L_{\mu}$ in (b) obtained from a single test run.

Figure 4.6(a) depicts all $D_{M}$ results obtained from the experiments. OBN gets higher scalability when the number of devices increases in a network group. For instance, when $t_{O B}=t_{B O}=5 \mathrm{~s}$ and $t_{M I}=30 \mathrm{~s}, D_{M}$ is $74 \%$ with 20 devices whereas it is $63 \%$ with 5 devices.

The effect of service timing is also detectable. For long $t_{O B}=t_{B O}$ values, $D_{M}$ decreases dramatically especially when $t_{M I}$ is low. When $t_{M I}$ gets higher, the effect of service timing slightly decreases. With 20 devices, for instance, when $t_{M I}=240 \mathrm{~s}, D_{M}$ ranges from $92 \%$ to $71 \%$ as $t_{O B}=t_{B O}$ is increased from $5 \mathrm{~s}$ up to $45 \mathrm{~s}$, respectively.

In addition, Figure 4.6(b) shows $L_{M}$ results obtained by different combinations of the service times, message frequencies, and number of devices. The low message frequency setups achieves high performances in terms of unidirectional average latency. Besides, the effect of service times gets more remarkable in high message frequency setups.

The overall conclusion of parameter testing is that it is possible to achieve reasonable data dissemination performance within a group of collocated devices. Nevertheless, the increase in the number of collocated devices does not help to achieve high performance when the number of messages are too high in the network.

\subsubsection{Small-Scale Networking Performance}

For the same number of devices $(N=10)$, the results of the experiments taken in a small-scale mobile network setup is given in Figure 4.7. The real-world tests are run only once for each combination between $t_{M I}=\{60 \mathrm{~s}, 120 \mathrm{~s}, 180 \mathrm{~s}, 240 \mathrm{~s}\}$ and $t_{O B}=t_{B O}=30 \mathrm{~s}$. The simulations are run 500 times for all parameter combinations. Figures 4.7(a) and 4.7(b) show the average data dissemination performances and average latency performances. Compared to the $D_{M}$ values of the simulations, $D_{\mu}$ of the real-world experiments are highly deviated. Nevertheless, the increase is consistent as $t_{M I}$ increases. In addition, the real-world $L_{\mu}$ values are in range of $L_{M} \pm L_{\sigma}$ values of the simulations. Overall, the small-scale networking performances show 
FIGURE 4.6

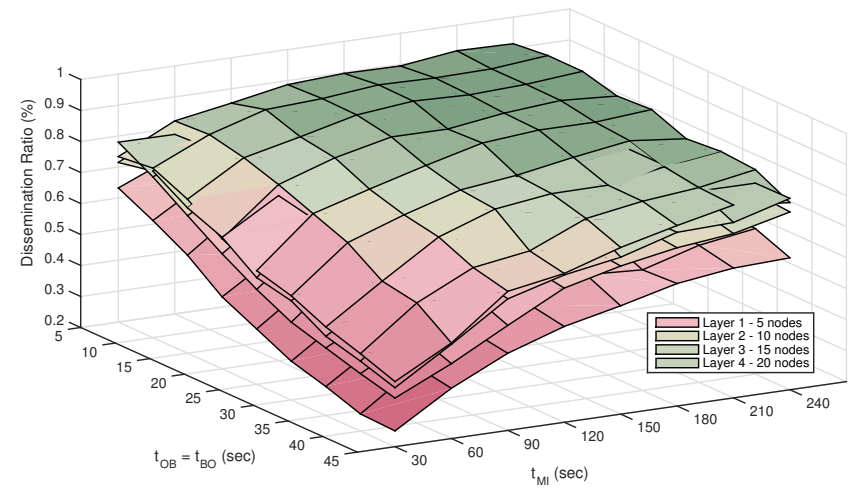

(a)

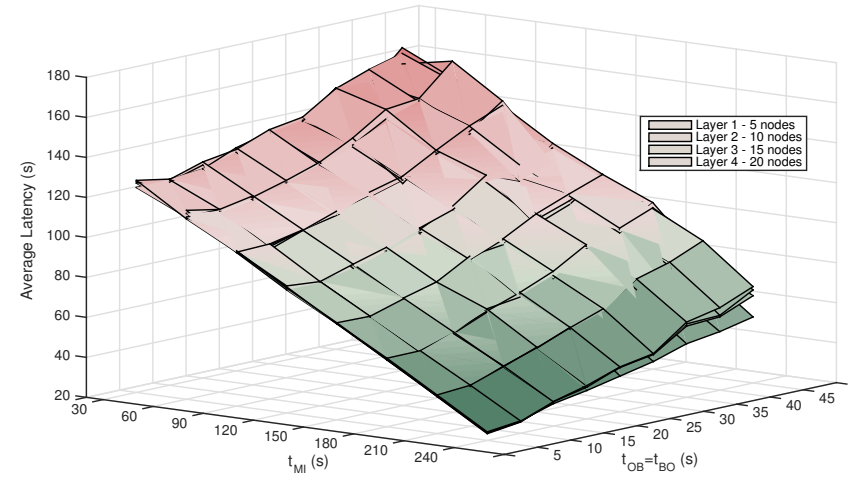

(b)

Parameter Testing Results

a very similar trend to the static networking results presented in Section 4.3.1. However, the negative effect of mobility on the performance is notable as well. The decrease in the $D_{M}$ values ranges between $\approx 10 \%$ and $\approx 20 \%$ for all tests. Besides, the increase in the $L_{M}$ values ranges between $\approx 30$ s and $\approx 90$ s for all tests.

\subsubsection{Large-Scale Networking Performance}

Figure 4.8 shows the data dissemination performances obtained from the 100-times repeated simulation runs for all combinations of model evaluation parameters. Overall, the results indicate that OBN achieves high scalability in dense deployments. As the densest setup, $N_{3}$ under $M_{1}$ gives $D_{M}$ of $72 \%$, with $D_{\sigma}$ of $9 \%$, when $t_{M I}=120$ s. For the same network setup, $D_{M}$ decreases to $52 \%$, with $D_{\sigma}$ of $5 \%$, when $t_{M I}$ is 60 s. 


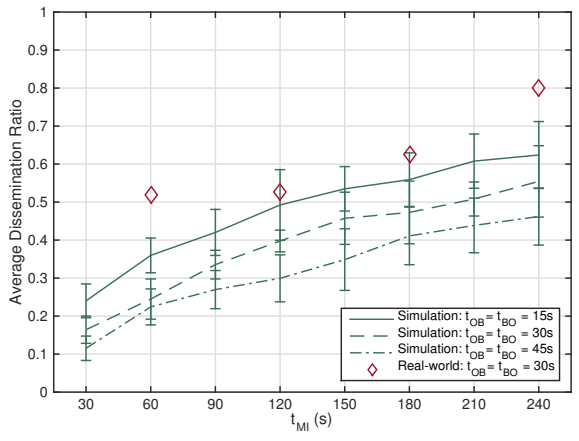

(a)

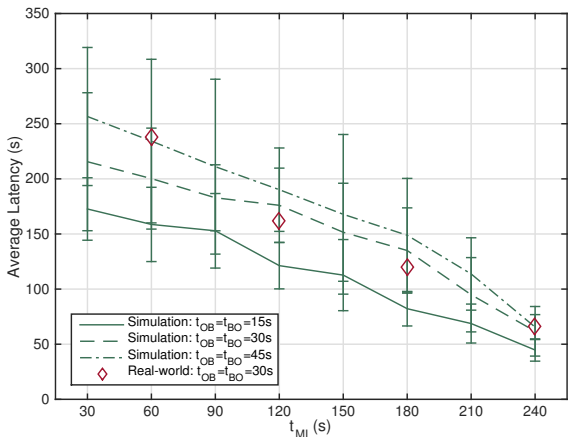

(b)

Small-scale Networking Results. Corresponding to different $t_{M I}$ settings, the plot lines indicate $D_{M}$ in (a) and $L_{M}$ in (b) whereas the ends of the error bars indicate $D_{\sigma}$ in (a) and $L_{\sigma}$ in (b) of the simulation results. The real-world results with $t_{O B}=t_{B O}=30 \mathrm{~s}$ are shown as points (boxes) corresponding to $t_{M I}=60 \mathrm{~s}, 120 \mathrm{~s}, 180 \mathrm{~s}$, and 240s. Each point represents a unique $D_{\mu}$ in (a) and $L_{\mu}$ in (b) obtained from a single test run.
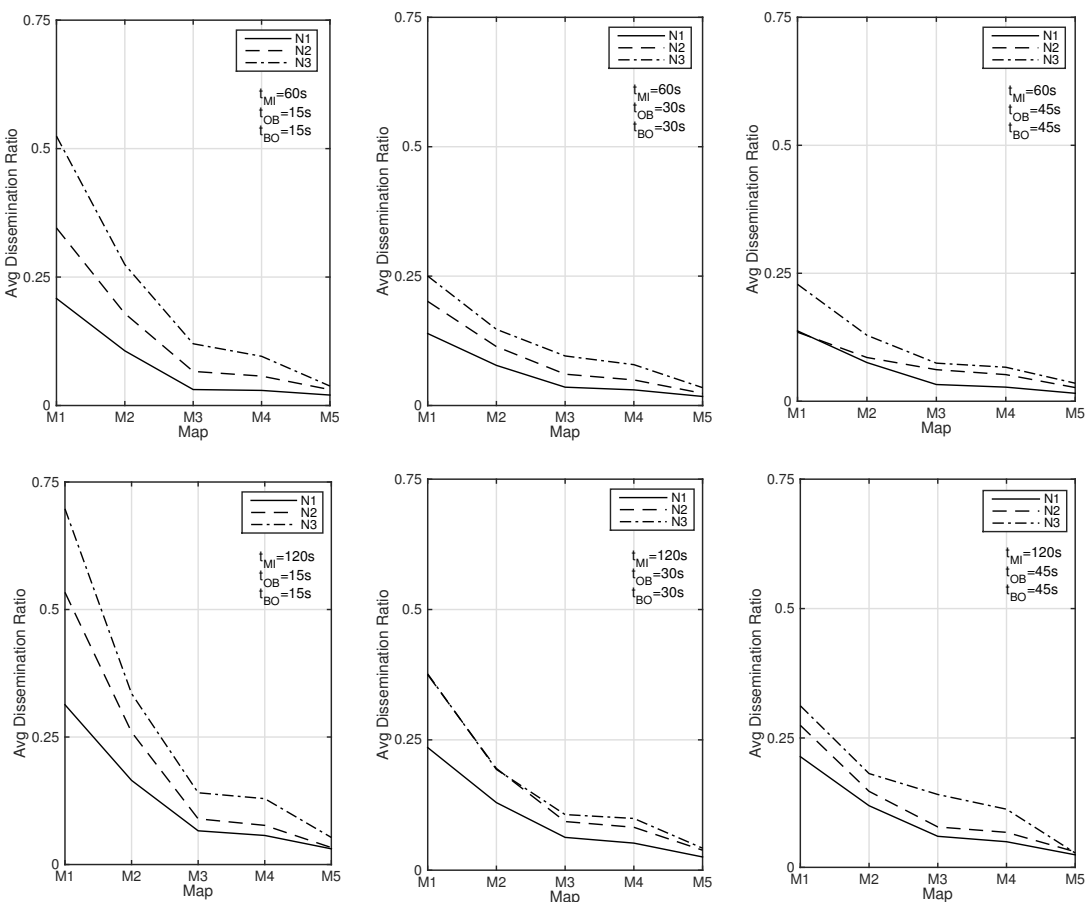

Large-Scale Networking Results 


\subsection{ENHANCEMENTS}

The presented protocol is based on predefined fixed service durations, i.e. $t_{O B}=t_{B O}$. Running the service automaton with random initialization times, $\mathrm{OBN}$ devices can be in either $\mathrm{OB}, \mathrm{BO}$, or U\&S states at a specific time. As a drawback of the protocol, devices running the same state cannot discover each other, e.g. OB-BO or BO-BO. Furthermore, if the periods of their services overlap, devices become less likely to discover each other. To reduce the possibility of same service conflicts (OB-OB and $\mathrm{BO}-\mathrm{BO})$, this section presents the following enhanced schemes:

- Message-adaptiveness: The OB-BO service times, i.e. $t_{O B}$ and $t_{B O}$, are adjusted based on the number of messages received.

- Time-adaptiveness: The BO service time of a device, i.e. $t_{B O}$, is adjusted based on the earliest deadline advertised by the OBs in its proximity.

- Dual radio utilization: An additional radio is utilized to complement the OB operation with scanning and the $\mathrm{BO}$ operation with advertisement.

\subsubsection{Message Adaptiveness}

This enhancement is based on adjusting $\mathrm{OB}$ and $\mathrm{BO}$ service times according to the number of received messages. More specifically, Algorithm 1 describes how message adaptiveness is provided over the OBN data switching example. The details are summarized as follows:

Algorithm 1. OBN with Message Adaptiveness

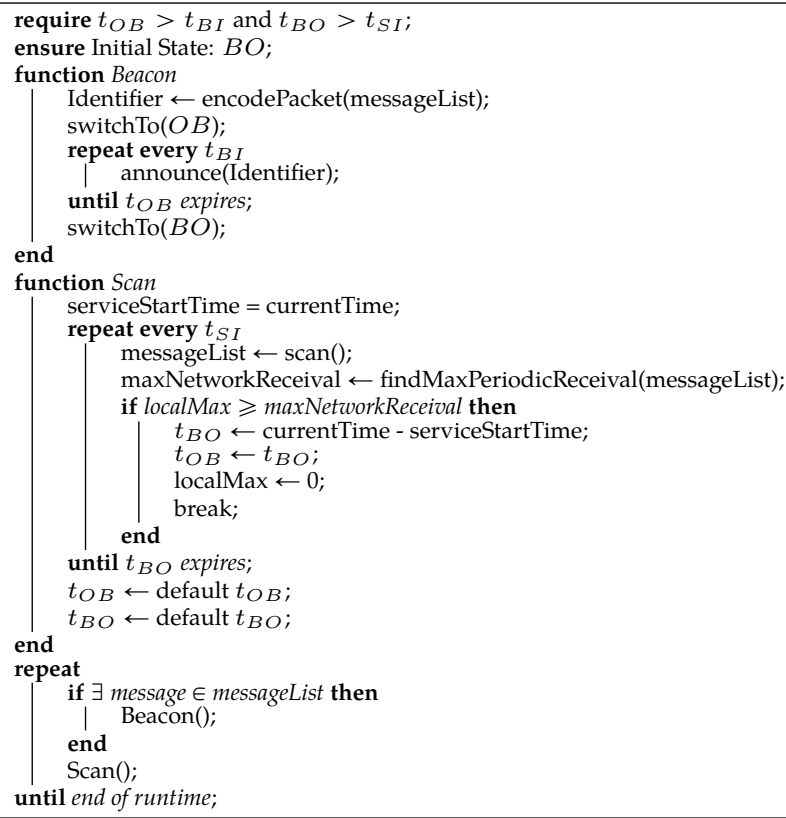


I) A device starts the automaton with predefined $t_{O B}$ and $t_{B O}$ which are equal to each other in the beginning. As the device functions in $\mathrm{OB}$ mode, it advertises the number of beacons it received during its previous BO period (shown as localMax). In this way, the other devices in its proximity can learn and adapt their BO service times according to this advertisement.

II) During the period of service, each $\mathrm{BO}$ runs an independent function called findMaxPeriodicReceival. This function runs in each scan and returns the maximum number of messages received in the network during the previous $\mathrm{BO}$ period. Each $\mathrm{BO}$ compares its localMax with this maximum. When this maximum is exceeded, the BO stops its service so as to start a new OB service.

III) In each period, localMax is reset to zero. In this way, this message-adaptive scheme forces a device to be adaptive in each period based on the ever-changing number of messages in its local network.

The message-adaptive scheme is helpful to reduce the redundant $\mathrm{BO}$ service times in a network, i.e. $t_{B O}$ is adjusted in each period. Moreover, $t_{O B}$ is also set equal to the latest $t_{B O}$. In this way, devices get more reactive to the number of messages shared in their locales. If the number of received messages increases, devices perform more frequent switches to increase the probability of switching different data among themselves. If loca lMax is not greater than the maxNetworkReceival, $t_{O B}$ and $t_{B O}$ are reset to their predefined default values.

\subsubsection{Time Adaptiveness}

This enhancement is based on adjusting $\mathrm{OB}$ and $\mathrm{BO}$ service times according to the advertised service deadlines. More specifically, Algorithm 2 describes how time adaptiveness is provided

Algorithm 2. OBN with Time Adaptiveness

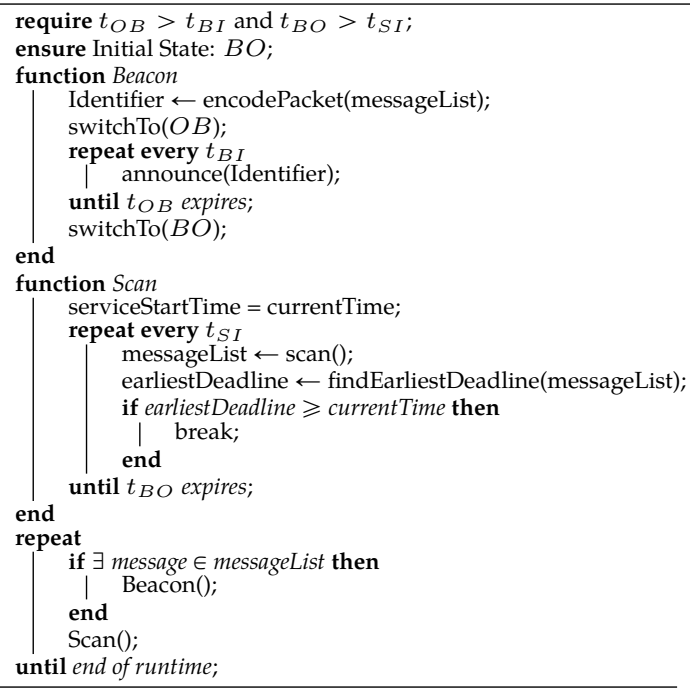


over the OBN data switching example. The details are summarized as follows:

I) A device starts the automaton with predefined $t_{O B}$ and $t_{B O}$ which are equal to each other in the beginning. As the device functions in $\mathrm{OB}$ mode, it advertises its current OB service deadline. In this way, BOs in its proximity can learn and adapt their service deadlines according to this advertisement.

II) During the period of service, each BO runs an independent function called findEarliestDeadline. This function runs in each scan and returns the earliest deadline received in the network. Each BO compares its own deadline with the earliest deadline. When earliest deadline is reached, the BO stops its service so as to start a new $\mathrm{OB}$ service.

The time-adaptive scheme is helpful to schedule OB-BO switches between devices. According to the algorithm, at least two devices can schedule their switching time. In this way, the redundant $\mathrm{BO}$ service times in a network are avoided. However, this scheme induces multiple BOs in the same environment to bound their services to the same deadline. That is, if an advertised deadline is received by a group of BOs, they will switch to OB mode at the same time, and data switching between them will never work assuming that their $t_{U \& S}$ are equal. To eliminate this possibility, the following measure is taken:

I) In each device, two flags are defined to indicate a scan mode: Discovery and Frequent. If a $\mathrm{BO}$ cannot find a $\mathrm{OB}$ during its service, the discovery flag is set. On the other hand, if the $\mathrm{BO}$ scans the same $\mathrm{OB}$ (or OBs) for two consecutive periods, the frequent flag is set.

II) Discovery mode assigns random $t_{B O}$ and $t_{O B}$, ranging from one-half shorter to one-half longer than the actual service time in our design. Here, the aim is to avoid OB-OB and BO-BO conflicts. Devices which cannot encounter any other device within their communication range extend their service times with the randomized deadlines.

III) Frequent mode enables a device to periodically switch between the $\mathrm{OB}$ and $\mathrm{BO}$ services with a duration relatively shorter, one-half shorter in our design, than the actual service time. Here, the aim is to increase the number of different deadline advertisements and discoveries between the devices which function in opposite services with the assumption that they will remain within range of each other.

\subsubsection{Dual Radio Utilization}

Today's smart mobile devices have two radios with beaconing support (e.g. Wi-Fi and BLE). To this end, dual radio utilization is offered as another type of protocol enhancement. Dual radio utilization is based on the concurrent use of beaconing and scanning operations. A device serving as an $\mathrm{OB}$ on the first radio can also perform scanning on the second radio. Conversely, a device serving as a $\mathrm{BO}$ on the first radio can also perform beaconing on the second radio. Figure 4.9 shows our approach of how packets are produced, disseminated, and interpreted with the utilization of two radios on a single device. 


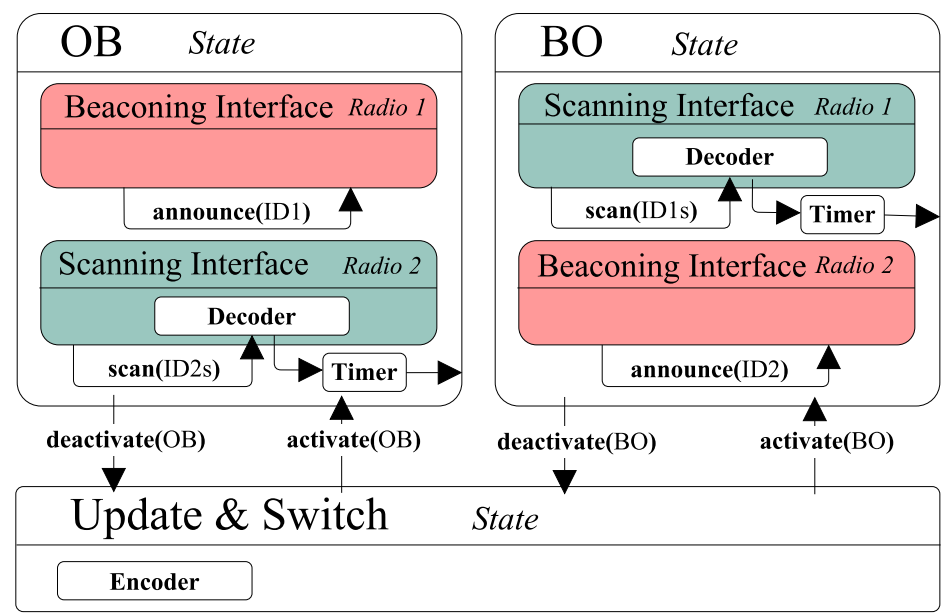

Finite state automaton with dual radio utilization

Running the automaton given in Section 4.1.2, the second radio is used to switch additional packets via its wireless network identifier (shown as $I D_{2}$ ). In this way, the scanning and beaconing functions are complemented in both of the $\mathrm{OB}$ and $\mathrm{BO}$ states.

It is possible to utilize the message-adaptive scheme and time-adaptive scheme together with the dual radios. For each wireless radio identifier, the advertisement fields required by the schemes must be encoded in both of the identifiers $\left(I D_{1}\right.$ and $\left.I D_{2}\right)$.

\subsubsection{COMPARISONS}

The performance of the presented enhancements are compared by means of simulations. The experimental setup is summarized in Table 4.5. The setup has four network scenarios: i) $S_{1}$ consists of 20 static devices $\left(N_{1}\right)$ that are all in range of each other. ii-iii-iv) $S_{2}, S_{3}$, and $S_{4}$ consist of mobile devices in different numbers $\left(N_{2}: 50, N_{3}: 100, N_{4}: 150\right.$, respectively) within a networking environment of $500 \mathrm{~m} \times 500 \mathrm{~m}$.

For each scenario, the simulation is repeated 100 times. The following protocol schemes are compared in terms of average data dissemination $\left(D_{M}\right)$, average dissemination latency $\left(L_{M}\right)$, and average number of hops: $i$ ) fixed duration, $i$ i) message-adaptive, $\left.i i i\right)$ time-adaptive, iv) message-adaptive with dual radio, $v$ ) time-adaptive with dual radio.

In the experiments, the wireless radio used is $\mathrm{Wi}-\mathrm{Fi}$, so that fields required by the enhanced protocols are encoded in the SSID fields together with the payload. For the protocols utilizing dual radio approach, BLE is used as the second radio. Since BLE UUID is short, only the fields required by the enhanced protocols are encoded, and payload is not included in the wireless identifier.

For each network setup, Figure 4.10 shows the average dissemination ratio $\left(D_{M}\right)$ and average latency $\left(L_{M}\right)$ results in two separate plots. Figure 4.11, on the other hand, shows the average delivery ratio $\left(R_{M}\right)$ and average number of hops $\left(H_{M}\right)$ in two separate plots. 
TABLE 4.5: Simulation Setup

\begin{tabular}{ll}
\hline Duration: 1800 s, Repeats: 100 \\
\hline Number of devices: $N_{1}=20, N_{2}=50, N_{3}=100, N_{4}=150$ \\
\hline Network scenarios: $S_{1}:$ Static, $S_{2}, S_{3}, S_{4}:$ Mobile \\
\hline Mobility Model: Random Waypoint & Movement pause $(s):[0,300]$ \\
\hline Wi-Fi range $(m): 50 \pm[0,25]$ & BLE range $(m): 40 \pm[0,20]$ \\
\hline$t_{O B}=t_{B O}=15 s$ & $t_{M I}(s)=60$ \\
\hline$t_{B I}, t_{S I}, t_{X O B}, t_{X B O}:$ Table 4.1 & \\
\hline$|Q|=10$ & \\
\hline
\end{tabular}

In the static setup composed of 20 devices $\left(N_{1}-S_{1}\right)$, it is noticeable that the proposed enhancements do not necessarily improve the OBN performance. The reason behind this is that the enhancements induce devices in range of each other to adapt their service deadlines based on a single advertised beacon majority of the time. If there are multiple of BOs which adapted their service times based on a single $\mathrm{OB}$ advertisement, the discovery between them becomes impossible in a static setup. On the other hand, collocated devices having same deadline increases. Therefore, the increased adaptation between a group of collocated devices still cannot overcome identical service conflicts (e.g. OB-BO, or BO-BO). On the other hand, with the default protocol which is based on fixed service durations and randomized switching periods, devices get more chance to overcome service conflicts.

In the setups involving mobility $\left(N_{2}-S_{2}, N_{3}-S_{3}\right.$, and $\left.N_{4}-S_{4}\right)$, the effect of each enhancement is remarkable. However, the performance gain is low compared to the default protocol based on fixed service durations. As shown in Figure 4.10 (a), the gain in $D_{M}$ is $\approx 3 \%$ for each enhancement. As shown in Figure $4.10(\mathrm{~b}), L_{M}$ is decreased $\approx 18$ s for each setup. In terms of end-to-end routing performance, similar observations are obtained. As shown in Figure 4.11(a), the gain in $R_{M}$ is $\approx 5 \%$ in $N_{3}-S_{3}$, and $N_{4}-S_{4}$. As shown in Figure 4.11(b), $H_{M}$ is slightly decreased in the mobile setups with the proposed enhancements. For all setups, the performance improvement obtained with dual radio utilization is very limited.

The overall conclusion is that the proposed enhancements perform better in mobile setups. They take advantage of mobility, so that the adaptations become open to different advertisements with different service timings. In this way, the identical service conflicts are decreased. 


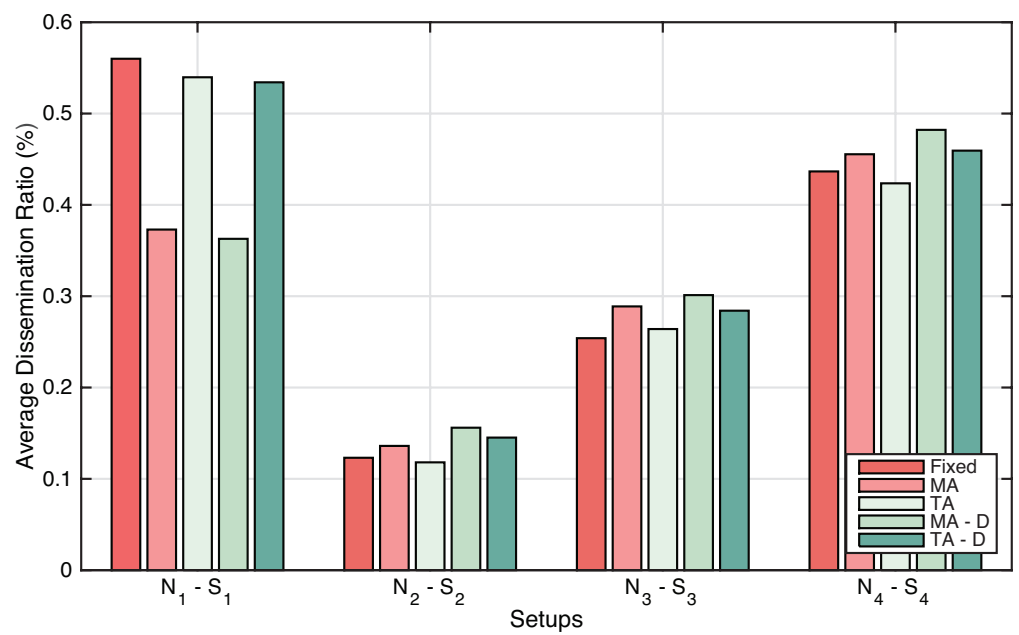

(a)

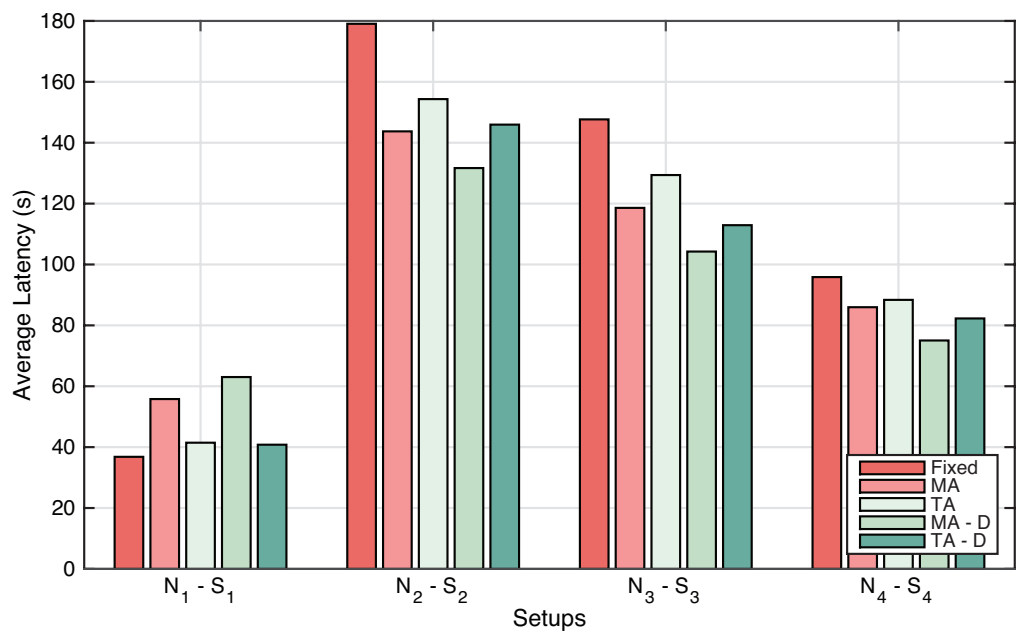

(b)

The data dissemination performance of the OBN protocols. Fixed: The default protocol, MA: Message-adaptive, TA: Time-adaptive, MA-D: MA with dual radio utilization, TA-D: TA with dual radio utilization 


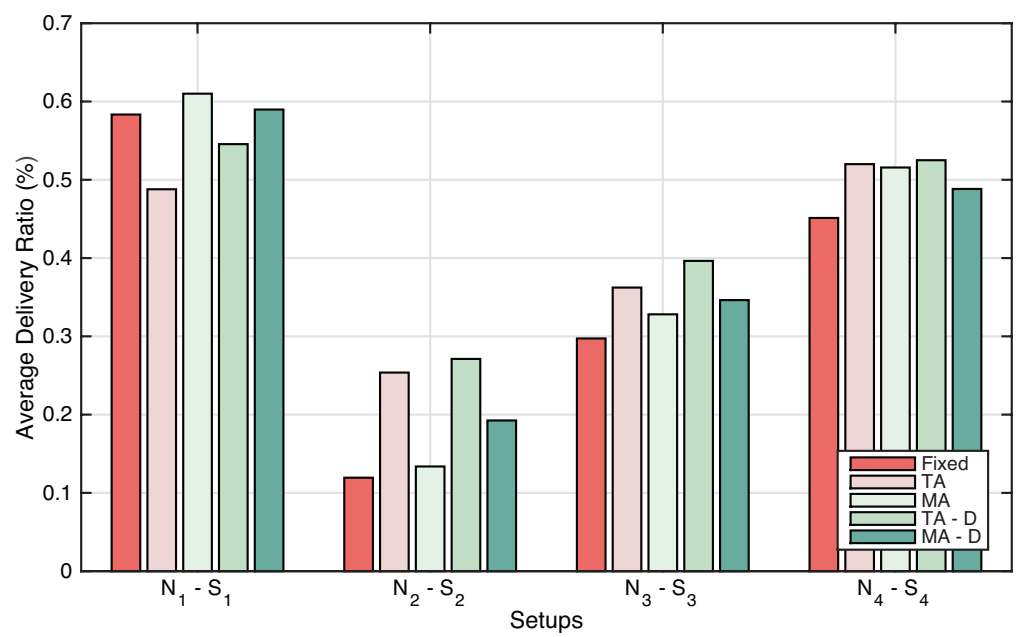

(a)

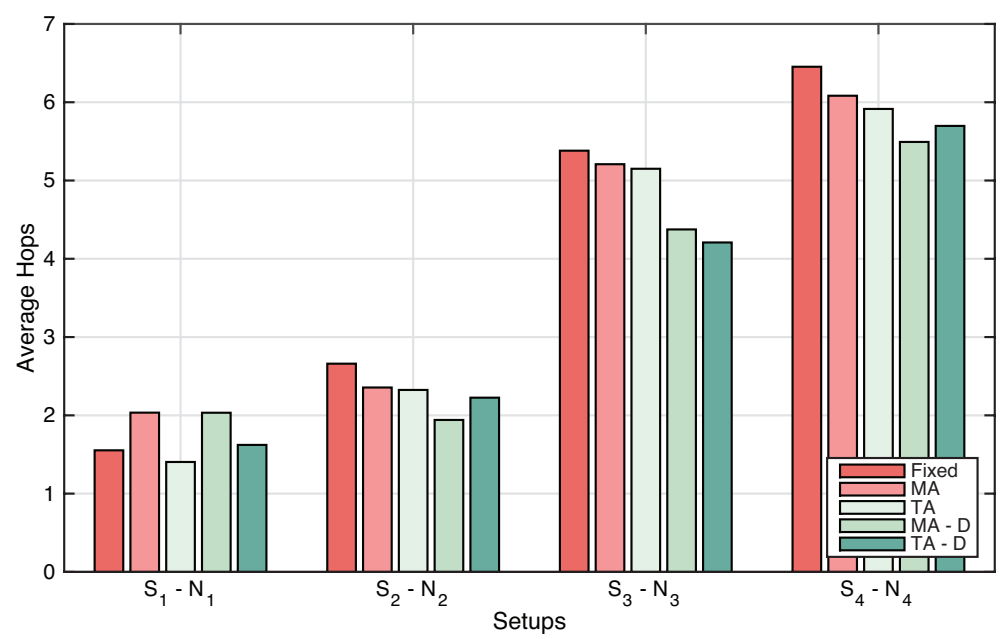

(b)

The end-to-end routing performance of the OBN protocols. Fixed: The default protocol, MA: Message-adaptive, TA: Time-adaptive, MA-D: MA with dual radio utilization, TA-D: TA with dual radio utilization 


\subsection{Concluding Remarks}

This chapter has presented the core networking model of the Cocoon architecture, called Opportunistic Beacon Networking (OBN). Compared to the traditional ad hoc networking techniques, $\mathrm{OBN}$ is a highly-opportunistic but a limited-throughput approach, i.e. it exploits wireless network identifiers short in length to provide information switching without connections. For an increased network throughput, it gains advantage from wireless broadcasts as well as from diversity and mobility of contacts. With OBN, an increase in the number of unique contacts can form a plethora of short messages in an OppNet. Under high mobility, multitude of messages can be disseminated over large areas in short time periods.

In spite of its versatility and energy efficiency, the OBN model contains certain limitations in its design. First of all, packet forwarding is one-directional-from an OB to BOs and therefore is subject to potential delays. Second, network throughput is extremely low, bound to the beacon frame length, which hinders sending large packets at once. Third, an OB can broadcast only one packet per cycle. To support two-way communications, a cycle-based data exchange protocol has been devised. To provide multi-packet transmissions, a queueing mechanism has been utilized.

Related to OBN, several model parameters have been investigated by means of both realworld experiments and verified simulations. The feasibility of the model has been validated. Besides, several protocol enhancements have been proposed to improve networking efficiency. Moreover, message aggregation techniques in identifier encoding are introduced for improved throughput. Our performance results have clearly shown that OBN-based protocols show high dissemination performance under high contact diversity and low data diversity. With its ease of use, OBN can be applied to a wide range of opportunistic communication use cases. 



\section{Applications}

WITH THREE DIVERSIFIED APPLICATION EXAMPLES, THIS CHAPTER INVESTIGATES the feasibility of the Opportunistic Beacon Networking (OBN) MOdel PRESENTED IN CHAPTER 4 IN DifFerent uRban scenarios. These applicaTIONS CAN BE USED FOR VARIOUS PURPOSES SUCH AS FOR COLLABORATIVE MESSAGING, SIMPLE GROUP COMMUNICATIONS, AND PARTICIPATORY MONITORING.

The First APPLICATION, OPPLINE, IS PROPOSED AS AN OPPORTUNISTIC SHORT MESSAGE SERVICE TO OPPORTUNISTICALLY CONNECT SEPARATED USERS IN A CROWDED AREA THROUGH A MULTI-HOP NETWORKING SETUP THAT CAN BE ACHIEVED BY EXPLOITING THE OTHER USERS AS ROUTERS. THE SECOND APPLICATION, BLESSED, IS PROPOSED AS A LIGHTWEIGHT DATA DISSEMINATION APPLICATION THAT SIMULTANEOUSLY UTILIZES DUAL-RADIO UTILIZATION PRESENTED IN SECTION 4.4.3 WITH A SPECIFIC BEACON ENCODING FOR AN INCREASED CONNECTIVITY IN A GROUP OF MOBILE DEVICES. THE THIRD APPLICATION, VADISS, IS PROPOSED AS AN OPPORTUNISTIC AD HOC DATA DISSEMINATION APPLICATION INTENDED FOR VEHICULAR ENVIRONMENTS TO COOPERATIVELY SPREAD ROAD CONDITIONS AND ONLINE TRAFFIC INFORMATION.

THROUGH DIFFERENT NETWORK SETUPS AND UNDER VARYING IN-NETWORK PARAMETERS, THE PERFORMANCE OF EACH PRESENTED APPLICATION IS STUDIED COMPREHENSIVELY. BASED ON THE RESULTS PRESENTED IN THIS CHAPTER, OBN CAN SERVE AS AN EFFICIENT MULTI-PURPOSE PROTOCOL IN ANY TYPE OF URBAN SCENARIOS. RANGING FROM SMALL-SCALE TO LARGE-SCALE NETWORKS, THIS CHAPTER SHOWS THAT OBN WITH SPECIALIZED APPLICATION IMPLEMENTATIONS IS SUITABLE FOR BOTH POINT-TO-POINT SHORT MESSAGING AND DATA DisSEMINATION PURPOSES. The RESUlts SHOW THAT OBN ACHIEVES REASONABLE PERFORMANCE FOR EACH APPLICATION. 


\subsection{Overview}

This chapter presents the design, implementation, and evaluation of three different applications built on top of the OBN model presented in Chapter 4. An OBN deployment can involve single (1) or multiple (N) sources and destinations based on the application requirements.

\subsubsection{Data Dissemination Applications}

As presented in Chapter 4, OBN is highly suitable for opportunistic data dissemination scenarios. In a multipoint-to-multipoint (N-to-N) OBN type, all participating devices can share data with each other. The performance results given in Chapter 4 clearly indicate that OBN achieves high scalability for $\mathrm{N}$-to-N scenarios under high device density and mobility. In addition, OBN may suit for the multipoint-to-point (N-to-1) scenarios having a central hub that continuously operates as a BO to collect messages from available OBs. To this end, OBN can be readily integrated to any group and type of smart mobile devices to render service to mobile sensing application types such as mobile lost \& found, participatory monitoring, proximity marketing, and so on. To prove our hypothesis, two different data dissemination applications are presented in Sections 5.3 and 5.4.

\subsubsection{Data Routing Applications}

Apart from data dissemination purposes, OBN can be utilized for data routing purposes. The performance results in Chapter 4 show that OBN provides high suitability for low message frequency scenarios, and for the networks having single (or limited number of) message sources. This simply means that OBN can be utilized in point-to-multipoint (1-to-N) and pointto-point (1-to-1) scenarios as well. To prove our hypothesis, an end-to-end message service, called Oppline, is proposed and tested in Section 5.2.

\subsubsection{Chapter Organization}

The rest of the chapter is organized as follows:

Section 5.2 presents Oppline, an ad hoc opportunistic short message service that can be collaboratively used by everyone who has a smart mobile device. The data exchange method of Oppline is built on top of the OBN model. The Oppline implementation is developed above the universal Wi-Fi standard, thus expedites platform-independent integration of related mobile applications for delay-tolerant communications in public space. Even in highlydense mobile networks of smart portable devices, Oppline gains performance from people's participation according to our experimental analysis validated with a real-life deployment. Without creating any network overhead, Oppline performs multi-hop message transmissions via short messages encoded in and decoded from the IEEE 802.11 SSID field. As a ubiquitous alternative to the situated communication systems, Oppline provides high versatility and usability for mobile ad hoc applications based on friend-to-friend messaging and data dissemination. 
Section 5.3 presents BLESSED, a universal opportunistic ad hoc networking model intended for smart mobile devices. It enables fast and lightweight data dissemination in wireless community networks through the complementary utilization of the IEEE 802.11 and BLE standards. As a ubiquitous alternative to the publicly-limited ad hoc networking interfaces, it resolves many of the peer-to-peer data forwarding issues with smart beacon advertisements. Opportunistic beacons of BLESSED require neither association nor connection for data sharing, instead exploit the network identifiers as message carriers. Our applicability tests on a real-life setup indicate the soundness of the model. Providing a high data dissemination performance, BLESSED can be applied to numerous daily application scenarios.

Section 5.4 presents an ad hoc opportunistic data dissemination protocol, called VADISS, that facilitates participatory traffic monitoring applications with smartphones. As a ubiquitous alternative to existing vehicular networking methods, VADISS uses the default Wi-Fi interfaces universally adopted by today's mobile devices. The routing is enabled with intermittent service advertisements and discoveries, and the data exchange is provided via built-in IEEE 802.11 beacon frames. VADISS orients neither association nor connection between devices, is not based on any road-side unit, and thus is readily available for vehicular end-use applications. Together with a smartphone-based online road monitoring application, VADISS has been tested in a real-traffic setup to assess the data dissemination performance. With the increasing coverage, quite promising efficiency has been reached, especially for routing of critical traffic information.

Figure 5.1 illustrates the presented applications on a city scale. Oppline is primarily intended for overcrowded areas. BLESSED can be used for data dissemination inside a group of people. VADISS is designed for ad hoc communications with smart mobile devices in traffic environments. These applications can be used for other various purposes such as for collaborative event detection, data traffic offloading, and participatory monitoring. Running the Cocoon architecture, these applications can run at the same time within a same OppNet.

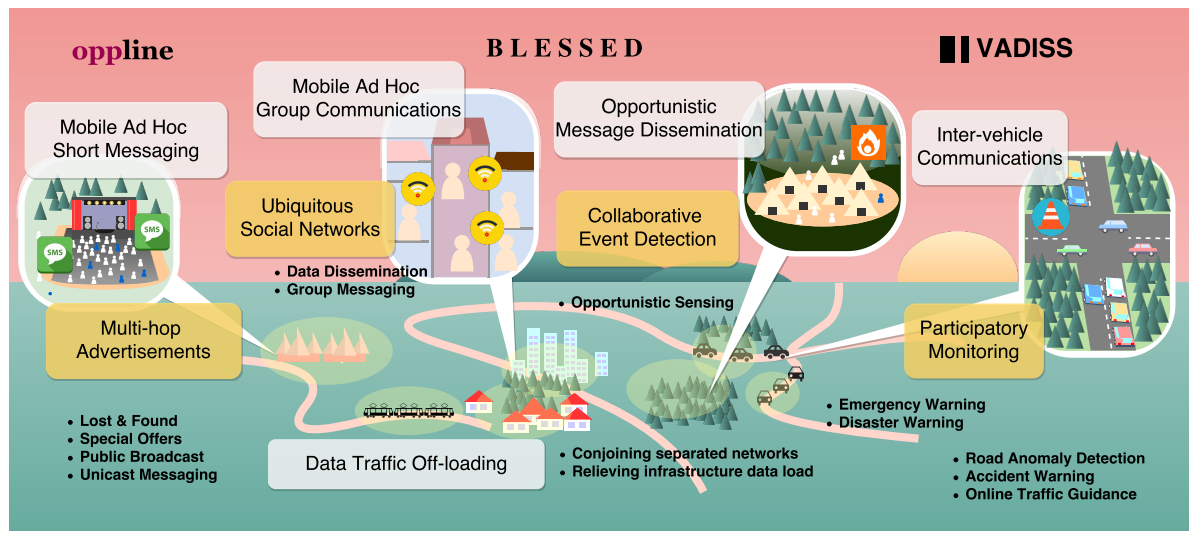

Application Scenarios 


\subsection{Oppline}

This section describes the design, implementation, and experimental analysis of Oppline, which stands for opportunistic line, an application that provides a new connectivity state between online and offline. Oppline is a smart mobile ad hoc networking service for short messaging between people at overcrowded events such as concerts, festivals, sports competitions, and more. During such events, people can get separated from their families or group of friends without any chance to regather. Besides, people are often confronted with internet access issues to reach their families or friends with their mobile handsets. From local networks (Bluetooth, Wi-Fi) to cellular short message service (SMS) to broadband GSM $(3 \mathrm{G}, 4 \mathrm{G})$, situated communication systems in crowds represent critical connectivity limits, and might sometimes be overloaded due to huge uplink/downlink demand from high number of mobile devices such as smartphones and tablets [130,33]. In contrast, Oppline benefits from a crowd of smart mobile devices to deliver short messages to and from group of people by using opportunistic Wi-Fi Service Set Identifier (SSID) broadcasts. For hopping of messages towards their destinations, it alternately functions SSID beaconing and scanning with the use of Wi-Fi Hotspot and Wi-Fi Infrastructure modes, respectively. Without IP layer connection, messages are encoded in SSID fields, are announced in Wi-Fi Hotspot mode, thus are directly delivered to the scanning devices in proximity. Devices employ a continuous beacon/scan switching to provide multi-packet multi-hop transmissions.

FIGURE 5.2
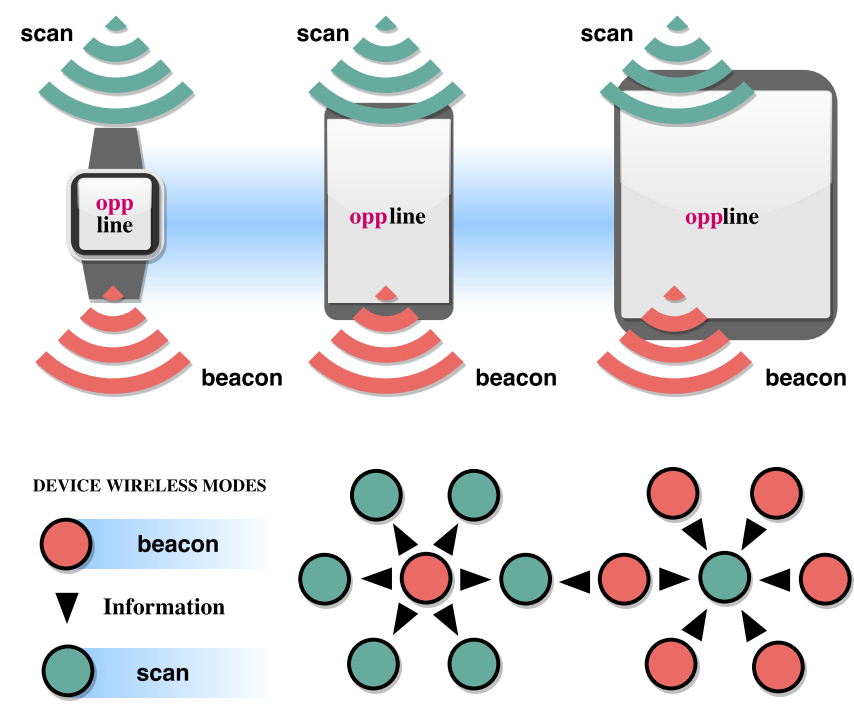

Oppline's data forwarding model 
As illustrated in Figure 5.2, Oppline is suitable for use on any kind of device supporting SSID beaconing, in particular smartphones, tablets, smartwatches that people always carry. It can run on top of any affiliated Wi-Fi adapter without any modification or installments on IEEE 802.11 standards. Moreover, it connects the diversity of smart mobile devices running different platforms such as Android, iOS, and Windows Phone. It is a bi-directional multi-hop networking model which does not get affected by network overhead. As an association-free protocol, it uses the wireless broadcast advantage. As Figure 5.2 delineates, multiple beacons can be received by a single scan operation and a beacon can be received by multiple scans at a single time. Thus, Oppline manages the distribution of multiple request and response messages between and through people without establishing connections. Even in dense networks, it facilely supports "friend finder" type opportunistic SMS and other daily-life SMS types as well as data dissemination services.

Oppline is evaluated with a comprehensive set of simulated network scenarios. The simulations are validated with the real-world experiments conducted with 20 Android smartphones. The feasibility of Oppline is investigated in terms of its end-to-end data delivery performance. From small-scale to large-scale, from sparse to dense, the unicast routing performance of Oppline is assessed through various network setups. The same setups are assessed for the multicast routing and broadcast routing efficiency as well. In each setup, different message creation intervals ranging between 1 minute to 15 minutes are studied with different number of devices ranging from 50 up to 200. According to our performance analysis, Oppline gains advantage from ubiquity of smart mobile devices to provide a reasonable endto-end connectivity in dense network environments.

\subsubsection{COMMUNicATION MODEL}

Our solution to the problem of decentralized opportunistic short message communications with smart mobile devices is based on a specific use-case of the Opportunistic Beacon Networking $(\mathrm{OBN})$ model previously presented in Chapter 4. Oppline operates the OBN model on top of the Wi-Fi standard to provide data switching with encoding of SSID fields. For brevity and clarity, Table 5.1 lists a set of notations used in the rest of the section.

For $d_{i}$ 's having at least one message, Oppline employs the finite state automaton presented in Chapter 3 with 3 states: Opportunistic Beacon (OB): Broadcast of encoded SSID fields in Wi-Fi Hotspot mode, Beacon Observer (BO): Scanning of SSID fields within proximity in Wi-Fi Infrastructure mode, and Update $\mathcal{E}$ Switch (U\&S): transition between OB and BO.

Oppline has a continuous duty cycling between the $\mathrm{OB}$ and $\mathrm{BO}$ states in order to provide two-directional data exchange. $U \& S$ is an idle state to switch between beaconing and scanning

TABLE 5.1: Notations

\begin{tabular}{ll}
\hline Symbol & Definition \\
\hline$d_{i}$ & $i$-th device in a network \\
\hline$N$ & Total number of devices in a network \\
\hline$M_{R E Q}$ & Total number of created requests in a network \\
\hline$M_{A C K}$ & Total number of created acknowledgments in a network \\
\hline$M$ & Total number of created messages in a network, $M_{R E Q}+M_{A C K}$ \\
\hline
\end{tabular}


functionalities, which is imperative for today's Wi-Fi adapters. At BO state, discovered SSIDs (messages), if any, are decoded. The received (and locally created) messages are stored in a circular queue, $Q$. To sustain multi-packet transmissions, the messages in $Q$ are selected for SSID encoding in a circular order in advance of each OB state. At each OB state, therefore, a different message is broadcast in the SSID field. If there is only one message in $Q, \mathrm{OB}$ state repeatedly broadcasts that one. If $Q$ is empty, the automaton stands by at $\mathrm{BO}$ mode until a message is discovered. The size of $Q$ can be determined based on network type or application needs. Since large queueing can cause message starvation, the size of $Q$ can be kept fixed, allowing the newest messages to overwrite the oldest ones.

Our design comprises two message types: Request ( $\mathrm{REQ}$ ) and Response (ACK). As Figure 5.3 demonstrates, any $d_{i}$ in a given network can send out a REQ towards a determined destination $d_{j}$ through other devices. Once $d_{j}$ receives the REQ, it immediately creates an ACK back to $d_{i}$. Relay devices in between $d_{i}$ and $d_{j}$ delete from their $Q$ the REQ of a discovered ACK since it is not necessary anymore. This also increases the probability of ACK relays in the duty cycles. Based on the contact opportunities and duty cycles of the devices in range of each other, the number of hops and paths can vary for each message created in the network.

\subsubsection{Model Parameters}

Each state of the automaton has specific service durations. The durations of $\mathrm{OB}$ and $\mathrm{BO}$ are $t_{O B}$ and $t_{B O}$, respectively, which can be adjusted based on the network conditions or application needs.

- At BO state, scan operation repeats in every $t_{S I}$.

- At OB state, beacon operation repeats transmitting of a packet in every $t_{B I}$.

- At U\&S state, activate and deactivate operations together defines the switching duration.

Transition from $\mathrm{OB}$ state to $\mathrm{BO}$ state, i.e. deactivating Wi-Fi Hotspot mode and activating Wi-Fi Infrastructure mode has a total duration of $t_{X B O}$. Transition from $\mathrm{BO}$ to OB, i.e. deactivating Wi-Fi Infrastructure mode and activating Wi-Fi Hotspot mode takes $t_{X O B}$ in total.

FigURE $5 \cdot 3$

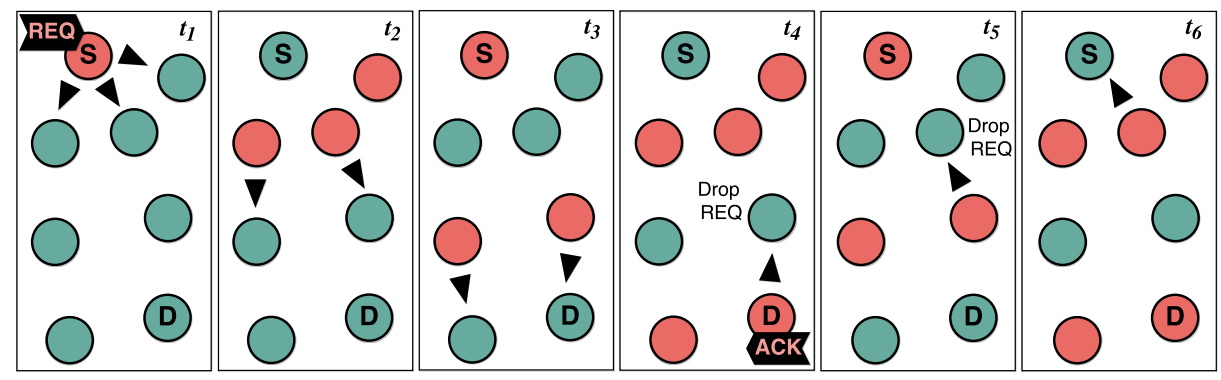

Data routing example 
Running the automaton with a random initialization time, a $d_{i}$ can be in either of three states at a particular time. In our design, $t_{O B}=t_{B O}$ for all $d_{i}$ 's in order to provide a fairness factor between the beacon and scan operations. If a group of $d_{i}$ 's in proximity of each other occurs at the same state with concurrent transitions, the data exchange will never work between them. Therefore, a randomization for $T$ for each $d_{i}$ is required to reduce the possibility of OB-OB and BO-BO conflicts which may abide forever. The randomization of $T$ is provided with non-deterministic $t_{X O B}$ and $t_{X B O}$ values.

\subsubsection{Message Encoding}

A default SSID field can contain at most 32 ASCII bytes. Based on the application requirements, Oppline messages can be designed in various compositions of routing-specific data. Figure 5.4 shows two SSID field encoding types of our design over five examples: i) a unicast message for end-to-end routing, and ii) a broadcast message for data dissemination. In order to encode values of several fields in less number of ASCII bytes, Base 94 conversion is applied since an SSID byte can have 94 different ASCII characters. The unicast message encoding type consists of 6 fields as shown in Figure 5.4(a):

1. Preamble (2 bytes): A distinctive tag to help BOs distinguish the message in an application or a network.

2. Message creation time (5 bytes): UNIX time is used in our design. However, shorter time formats such as HHmm converted to Base 94 can also be used for compactness in exchange for less timing precision.

3. $R E Q / A C K$ type (1 byte): A predefined type of a request or a response that represents a particular message or context.

4. Location information ( 8 bytes): The position of or information about the location where the message is created. GPS is used in our design. For indoor applications, the location can be reported by user input.

5. Source Device Identifier (8 bytes): MAC address is used in our design. An alternative such as device ID, username, and so on can also be used instead.

6. Destination Device Identifier (8 bytes). Same as (5).

A broadcast message encoding, on the other hand, may discard routing-related fields such as device identifiers in order to gain length for the message field to be publicly disseminated. The examples for this encoding is shown in Figure 5.4(b).

Apart from these encoding types, Figure 5.4(c) and Figure 5.4(d) show SSID examples that comply with the Cocoon beacon format presented in Section 3.3.2. In these examples, the first 12 bytes are reserved for the architecture use, the remaining bytes are used for the Oppline application. As can be seen in these examples, the reserved fields such as UNIX timestamp and QoS (CS, CD, DS, DD) are untouchable by the application. Yet, the routing objective of the application can be defined as a field defined in the payload. Apart from the reserved routing field, the payload can contain additional routing fields. 
FigURE 5.4

\begin{tabular}{|c|c|c|c|c|c|c|c|}
\hline Fields & $\begin{array}{c}\text { App } \\
\text { ID }\end{array}$ & $\begin{array}{c}\text { Message } \\
\text { Creation Time }\end{array}$ & $\begin{array}{l}\text { REQ/AC } \\
\text { Type }\end{array}$ & \multicolumn{2}{|c|}{$\begin{array}{l}\text { Location } \\
\text { Information }\end{array}$} & $\begin{array}{l}\text { Source Device } \\
\text { Identifier }\end{array}$ & $\begin{array}{l}\text { Relay Device } \\
\text { Identifier }\end{array}$ \\
\hline Example & $\begin{array}{l}\text { Preamble } \\
\qquad \text { [c }\end{array}$ & $\begin{array}{l}\text { UNIX Timestamp } \\
1558245655\end{array}$ & $\begin{array}{l}\text { p "Come } \\
\text { back" }\end{array}$ & $\begin{array}{l}\text { GPS Latitude } \\
4129260 \times 10^{-5}\end{array}$ & $\begin{array}{l}\text { GPS Longitude } \\
3633720 \times 10^{-5}\end{array}$ & $\begin{array}{c}\text { MAC Address } \\
01: 23: 45: 67: 89: \mathrm{AB}\end{array}$ & $\begin{array}{c}\text { MAC Address } \\
\mathbf{F F}: \mathbf{F F}: \mathbf{F F}: \mathbf{F F}: \mathbf{F F}: \mathbf{F F}\end{array}$ \\
\hline Encoding & ASCII & $\begin{array}{l}(1558245655)_{10} \\
\text { to Base } 94\end{array}$ & ASCII & $\begin{array}{c}(4129260)_{10} \\
\text { to Base } 94\end{array}$ & $\begin{array}{c}(3633720)_{10} \\
\text { to Base } 94\end{array}$ & $\begin{array}{c}(0123456789 \mathrm{AB})_{16} \\
\text { to Base } 94\end{array}$ & $\begin{array}{c}\text { (FFFFFFFFFFFF) } 16 \\
\text { to Base } 94\end{array}$ \\
\hline Packet & [ $\mathrm{c}$ & \begin{tabular}{|l|l|l|l}
$J$ & $f$
\end{tabular} & f 7 & \begin{tabular}{|l|l|l}
4 & $\mathrm{~S}$ \\
\end{tabular} & \begin{tabular}{|l|l|l|l|l}
4 & $Z$ & $M$ & \\
\end{tabular} & 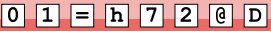 & 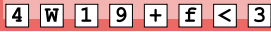 \\
\hline
\end{tabular}

(a) Unicast message encoding example regardless of the Cocoon beacon format. As the same field presented for the Cocoon beacon format, the preamble defines the application type and priority. In this example, the preamble tells that the following bytes are designed for unicast messaging. Therefore, the timestamp field holds the message creation time. The message type is an REQ from a source. Additionally, GPS location is encoded. For routing, the source and destination are included.

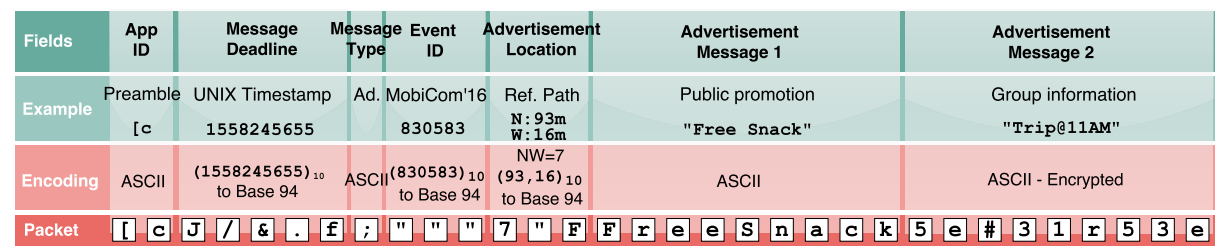

(b) Broadcast message encoding example regardless of the Cocoon beacon format. As the same field presented for the Cocoon beacon format, the preamble defines the application type and priority. In this example, the preamble tells that the following bytes are designed for broadcast messaging. Therefore, the timestamp field holds the message deadline time. The message type is an advertisement. The advertisement event is encoded which can must be known beforehand. The advertisement location is encoded with regard to the event reference point. Two advertisements (the latter is encrypted) are encoded in the remaining bytes.

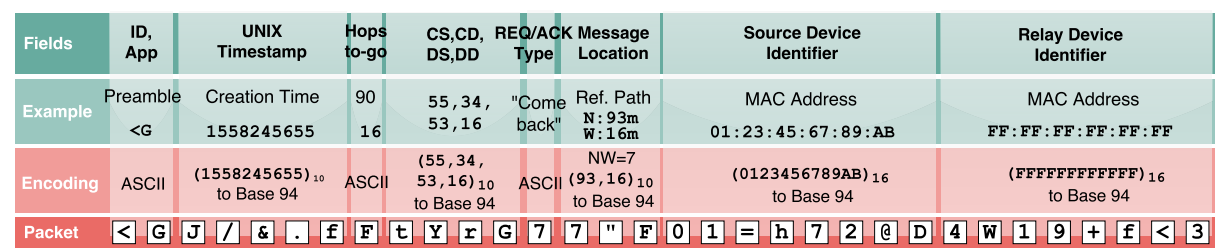

(c) Unicast message encoding example complying with the Cocoon beacon format. The reserved fields hold the application type, message creation time, the number of remaining hops-to-go, and current QoS information. The payload is used for the message type, location, source, and destination.

\begin{tabular}{|c|c|c|c|c|c|c|c|c|}
\hline Fields & $\begin{array}{l}\text { ID, } \\
\text { App }\end{array}$ & $\begin{array}{l}\text { UNIX } \\
\text { Timestamp }\end{array}$ & $\begin{array}{l}\text { Hops } \\
\text { to-go }\end{array}$ & $\begin{array}{l}\text { CS,CD, } \\
\text { DS,DD }\end{array}$ & $\begin{array}{c}\text { Message } \\
\text { Type }\end{array}$ & \multicolumn{2}{|c|}{$\begin{array}{c}\text { Advertisement } \\
\text { Location Information }\end{array}$} & $\begin{array}{l}\text { Advertisement } \\
\text { Message }\end{array}$ \\
\hline Example & $\begin{array}{c}\text { Preamble } \\
\text { ]c }\end{array}$ & $\begin{array}{c}\text { Deadline } \\
1558245655\end{array}$ & $\begin{array}{c}90 \\
3\end{array}$ & $\begin{array}{l}55,34 \\
53,16\end{array}$ & Ad. & $\begin{array}{c}\text { GPS Latitude } \\
4129260 \times 10^{-5}\end{array}$ & $\begin{array}{l}\text { GPS Longitude } \\
3633720 \times 10^{-5}\end{array}$ & $\begin{array}{l}\text { Crowd Information } \\
\text { "Lost Child" }\end{array}$ \\
\hline Encoding & ASCII & $\begin{array}{l}(1558245655)_{10} \\
\text { to Base } 94\end{array}$ & ASCII & $\begin{array}{l}(55,34 \\
53,16)_{10} \\
\text { to Base } 94\end{array}$ & ASCII & $\begin{array}{c}(4129260)_{10} \\
\text { to Base } 94\end{array}$ & $\begin{array}{l}(3633720)_{10} \\
\text { to Base } 94\end{array}$ & ASCII - Encrypted \\
\hline Packet & ] $\mathrm{c}$ & $\begin{array}{lll}\mathrm{J} & \boldsymbol{E}\end{array}$ & 3 & $t \quad Y \quad r$ & G 7 & \begin{tabular}{|l|l|l|l} 
& $?$ & $\mathrm{U}$ \\
\end{tabular} & \begin{tabular}{|l|l|l|l}
4 & $\mathbf{Z}$ & $\mathbf{M}$ & $\mathrm{u}$ \\
\end{tabular} & 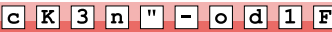 \\
\hline
\end{tabular}

(d) Broadcast message encoding example complying with the Cocoon beacon format. The reserved fields hold the application type, message creation time, the number of remaining hops-to-go, and current QoS information. The payload is used for an encrypted advertisement encoded with its GPS location.

Message encoding examples 


\subsubsection{IMPLEMENTATION \& EVAluation}

This sub-section gives the implementations, experiments, model evaluation parameters and metrics, and performance analysis.

\section{Mobile Application}

Oppline is implemented as an Android application for testing purposes. The application employs the basic OBN protocol given in Chapter 4 with the utilization of Wi-Fi Hotspot and Wi-Fi Infrastructure modes in an alternating manner for the $\mathrm{OB}$ and $\mathrm{BO}$ roles, respectively. As defined in the protocol, Wi-Fi Inrastructure mode constantly runs unless a message is created or scanned. If a message is available, SSID is encoded to that message in advance of beaconing with Wi-Fi Hotspot mode. In each duty cycle, the application selects the frontmost packet of $Q$.

The mobile application tests are held with various networks formed of Samsung S4 Mini and Motorola Moto $G$ phones. A total of 20 smartphones are utilized in order to collect time measurements of Wi-Fi operations used in the model as well as to investigate Oppline's networking performance in reality. The measurements are further used in the simulation modelling. On the other hand, the networking results are compared with that of the simulation runs in order to verify the simulator.

\section{Simulator}

In addition to the mobile application, the Oppliqué simulator presented in Appendix B is used. In brief, Oppliqué is a cycle-based simulator is implemented in Matlab that can run different OBN schemes for any kind of network setup, including the Oppline's data exchange protocol. The simulator creates messages in a given network setup as discrete events. At a particular instant in time, each message is created within a simulated device (source). Devices mimic the data exchange protocol with specified network parameters.

The simulator is run with an abstract Wi-Fi PHY/MAC modelling. In reality, several Wi-Fi operations have uncontrollable execution times. The simulator is fed $t_{X O B}, t_{X B O}$, $t_{B I}$, and $t_{S I}$ values collected from all of the mobile application runs. Table 5.2 demonstrates the average and standard deviation values of these values. Out of all real-world measurement values, simulated devices pick a random group of $t_{X O B}, t_{X B O}, t_{B I}$, and $t_{S I}$ for each of their duty cycle.

TABLE 5.2: Real-world durations used in the simulator

\begin{tabular}{|c|c|c|c|}
\hline Symbol & Duration & Symbol & Duration \\
\hline$t_{B I}$ & $\mu=0.100 \mathrm{~s}, \sigma=0.014 \mathrm{~s}$ & $t_{S I}$ & $\mu=3.000 \mathrm{~s}, \sigma=0.247 \mathrm{~s}$ \\
\hline$t_{X O B}$ & $\mu=4.302 \mathrm{~s}, \sigma=0.524 \mathrm{~s}$ & $t_{X B O}$ & $\mu=3.407 \mathrm{~s}, \sigma=0.327 \mathrm{~s}$ \\
\hline
\end{tabular}




\subsubsection{Simulation Validation}

To investigate its regularity regarding the data exchange protocol, the simulator is compared with the mobile application runs conducted in a real-world network deployment. Both realworld experiments and simulations are run with the same varying model parameters in order to find a correlation between their performance outcomes. Real-world experiments consist of 20 phones that are situated in range of each other. The simulation is set up in the same manner with the same number of devices. All tests are performed under no mobility. Thus, it is aimed to discover the accuracy of the simulation model when mobility effect is discarded.

The real-world experiments and simulations are run 3 times and 500 times, respectively, per unique parameter combination. Figure 5.5 shows the collated results for data dissemination performance and end-to-end latency performance of the model. Under different message creation intervals (shown as $t_{M I}$ ) settings ranging from $30 \mathrm{~s}$ up to $240 \mathrm{~s}$, the real-world test results and the simulation test results are shown together for two different $t_{O B}=t_{B O}$ settings, $25 \mathrm{~s}$ and $45 \mathrm{~s}$. For all network settings, the simulation results are in line with the realworld test results. Nevertheless, minor deviations are notable especially for the latency results. These deviations might be related either to the limited number of the physical world test runs, or to the inimitable environmental factors affecting the PHY/MAC operations in reality. Nonetheless, it is evident that the simulation results highly correlate with the corresponding results obtained from real-world experiments. The simulator is used for the evaluation of our test setups.

FIGURE 5.5

Simulation: $\mathrm{t}_{\mathrm{OB}}=\mathrm{t}_{\mathrm{BO}}=25 \mathrm{~s} \longrightarrow$ Simulation: $\mathrm{t}_{\mathrm{OB}}=\mathrm{t}_{\mathrm{BO}}=45 \mathrm{~s} \bullet$ Real-world: $\mathrm{t}_{\mathrm{OB}}=\mathrm{t}_{\mathrm{BO}}=25 \mathrm{~s} \quad$ Real-world: $\mathrm{t}_{\mathrm{OB}}=\mathrm{t}_{\mathrm{BO}}=45 \mathrm{~s}$
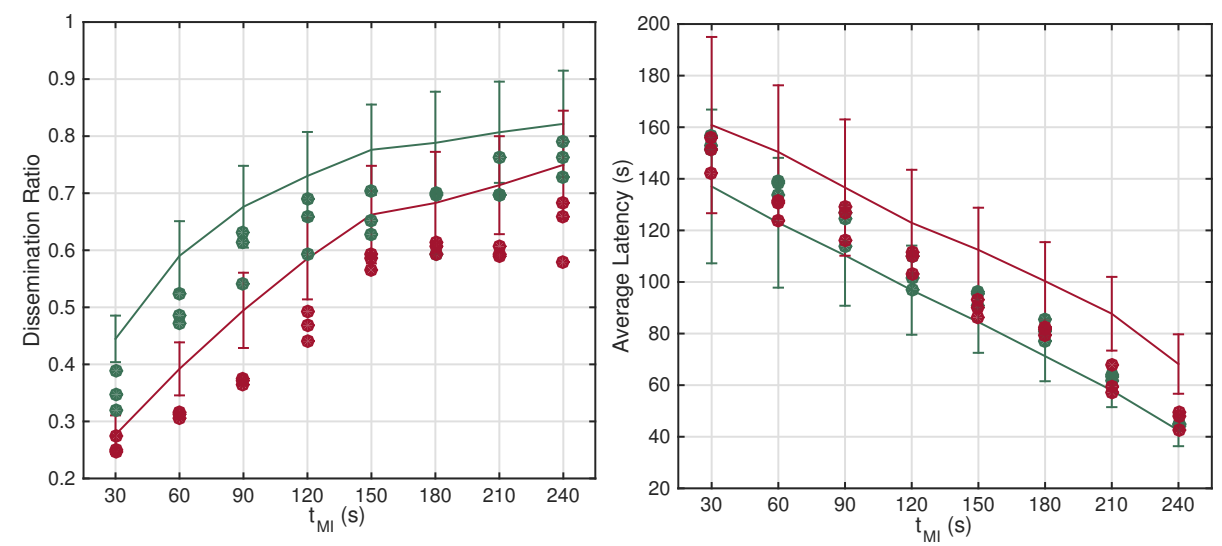

Simulation validation test results grouped under different $t_{M I}$ settings. For each $t_{M I}$, the realworld results are shown as a collection of 3 runs. For the simulations, the averaged results of 500 runs are represented. The end of the error bars indicate the standard deviation values. In all tests, $|Q|=10$. 


\subsubsection{Test Setups \& Model Evaluation Parameters}

All tests are conducted by means of simulations with a set of controlled experiments based on varying network types and model evaluation parameters. In practice, our aim is two-fold:

i) to assess Oppline's performance under varying network densities. 4 different network groups are formed: $N_{1}, N_{2}, N_{3}$, and $N_{4}$ consist of 50, 100, 150, and 200 devices, respectively. Besides, 3 different deployments are formed with the following network sizes: $S_{1}: 500 m \times 500 m, S_{2}: 750 m \times 750 m$, and $S_{3}: 1000 m \times 1000 m$.

The tests are taken for all possible combinations between the network groups/deployments.

ii) to assess Oppline's performance under varying message numbers. 6 different values for message creation interval $\left(t_{M I}\right)$ are tested, ranging from $60 \mathrm{~s}$ up to $900 \mathrm{~s}$.

The tests with different $t_{M I}$ are taken for all combinations in (i).

Each network setup is repeated for 100 times. Table 5.3 shows the simulation parameters in brief. For the device movements, random waypoint mobility model with movement pauses is utilized. The devices are given random radio ranges uniformly ranging from $25 \mathrm{~m}$ to $75 \mathrm{~m}$.

\subsubsection{Evaluation Metrics}

In a unique network run, let us define $M^{+}$as the number of delivered messages out of $M$, $M^{+}<M$. Same definition holds for $M_{R E Q} \rightarrow M_{R E Q}^{+}$and $M_{A C K} \rightarrow M_{A C K}^{+}$. Similarly, let us define $M^{*}$ as the total number of message copies out of $M$ messages created in $d_{i}$ 's. The network setups are evaluated with the following metrics:

1. Message reception rate (PRR), calculated as $\frac{M^{+}}{M}$.

The PRR of REQS $\left(\mathrm{PRR}_{R E Q}\right)$ is calculated as $\frac{M_{R E Q}^{+}}{M_{R E Q}}$.

The PRR of ACKs $\left(\mathrm{PRR}_{A C K}\right)$ is calculated as $\frac{M_{A C K}^{+}}{M}$.

2. Latency $(L)$, the delivery time of a message between its source and destination. $L_{R E Q}$ is the sum of the delivered REQ latencies, divided by $M_{R E Q}^{+} . L_{A C K}$ is the sum of the delivered ACK latencies, divided by $M_{A C K}^{+}$.

3. Average dissemination ratio $(D)$, calculated as $\frac{M^{*}}{M \times(N-1)}$.

TABLE 5.3: Simulation Parameters

\begin{tabular}{|c|c|}
\hline \multicolumn{2}{|l|}{ Duration: 14400s, Repeats: 100} \\
\hline \multicolumn{2}{|c|}{ Number of devices: $N_{1}=50, N_{2}=100, N_{3}=150, N_{4}=200$} \\
\hline \multicolumn{2}{|c|}{ Networks $(m \times m): S_{1}=500 \times 500, S_{2}=750 \times 750, S_{3}=1000 \times 1000$} \\
\hline Mobility Model: Random Waypoint & Movement pause $(s):[0,1800]$ \\
\hline \multicolumn{2}{|l|}{ Wi-Fi range $(m): 50 \pm[0,25]$} \\
\hline \multicolumn{2}{|l|}{$t_{O B}=t_{B O}=15 \mathrm{~s}$} \\
\hline$t_{B I}, t_{S I}, t_{X O B}, t_{X B O}:$ Table 5.2 & $t_{M I}(s)=\{60,180,360,540,720,900\}$ \\
\hline$|Q|=10$ & \\
\hline
\end{tabular}




\subsubsection{Performance Analysis}

The results of the experiments are presented in this sub-section.

Figure 5.6 shows the unicast data delivery performance results grouped under $S_{1}, S_{2}$, and $S_{3}$. In each group, $N_{1}, N_{2}, N_{3}$, and $N_{4}$ are shown as separate plots. In each plot, the average PRR values for $M, M_{R E Q}$, and $M_{A C K}$ are shown as separate line graphs demonstrated on a $t_{M I}$ scale.

In Figure 5.6(a), the increase in the average PRR values is quite remarkable as $t_{M I}$ increases. In other words, any deployment can handle data delivery more efficiently when the number of messages decreases in the network. When $t_{M I}=60 \mathrm{~s}, \mathrm{PRR}_{M}$ is $19 \%$ for $N_{1}$, gradually increases as $N$ increases, and reaches to $57 \%$ for $N_{4}$. The difference in $\mathrm{PRR}_{M}$ between $t_{M I}=60 \mathrm{~s}$ and $t_{M I}=360 \mathrm{~s}$ is $\approx 25 \%$ for $N_{1}$. This difference ranges between $\approx 30 \%$ and $\approx 40 \%$ for $N_{2}, N_{3}$, and $N_{4}$. These results simply indicate the positive effect of high device numbers on the delivery performance. When $t_{M I}=900 \mathrm{~s}$, all $N$ setups except $N_{1}$ attain a $\mathrm{PRR}_{M}$ of more than $\approx 85 \%$. For $N_{4}$, which is the densest setup of our experiments, a $\mathrm{PRR}_{M}$ of $93 \%$ is achieved as the maximum. In Figure 5.6(b), in which the PRR values of the $S_{2}$ setups are depicted, a similar trend is present as in $S_{1}$. Nevertheless, since the network size is bigger, the decrease in the overall delivery performance for the same $N$ setups is also remarkable. In comparison to $\mathrm{PRR}_{M}$ values obtained in $S_{1}$, that of in $S_{2}$ are from $\approx 15 \%$ to $35 \%$ lower when $t_{M I}=60 \mathrm{~s}$. Of $\mathrm{PRR}_{M}$ on the scale of $t_{M I}$ ranging between $360 \mathrm{~s}$ and $900 \mathrm{~s}$, the difference in the overall performance between the $S_{1}$ and $S_{2}$ setups decreases to $\approx 20 \%$. A correlative decrease in the overall delivery performance for the same networks deployed in $S_{3}$ is shown in Figure 5.6(c). Compared to the $S_{2}$ results, there exists an average of $12 \%$ drop in $\mathrm{PRR}_{M}$ for all of the results obtained in $S_{3}$ setups.

In all tests, the $\mathrm{PRR}_{A C K}$ values which demonstrate the average round trip delivery efficiency are roughly $50 \%$ lower than their corresponding $P_{R R} R_{R E}$ values. This means that only approximately half of the sources in all setups can get a response back from their destinations. On the other hand, the $\mathrm{PRR}_{R E Q}$ values follow a high performance in parallel with the $\mathrm{PRR}_{M}$ values in all tests. Note that $M=M_{R E Q}+M_{A C K}$ and $M_{A C K}=M_{R E Q}^{+}$. In any setup, $M$ is influential on $\mathrm{PRR}_{R E Q}$, and consequently $\mathrm{PRR}_{R E Q}$ is influential on $\mathrm{PRR}_{A C K}$. Since $\mathrm{REQS}$ of the discovered ACKs are dropped in the device buffers, $L_{A C K}$ performs better than $L_{R E Q}$ and $L_{M}$ as Figure 5.7 depicts for $S_{1}$. Overall, $L_{M}$ significantly decreases as $N$ and/or $t_{M I}$ increases. For low $t_{M I}$, it is shown that a message is delivered in average of $600 \mathrm{~s}$ for $N>50$. For $t_{M I}>540 \mathrm{~s}$, the average latencies fall below 400s. For $t_{M I}=900 \mathrm{~s}$, all latencies are around 180s. From the latency results, it is also possible to assess the round trip time (RTT) of the delivered REQ-ACK pairs. $L_{R E Q}+L_{A C K}$ gives the average RTT. In all network setups except $N_{1}$, the RTT ranges between 320s (for $t_{M I}=900 \mathrm{~s}$ ) up to 780s (for $t_{M I}=60 \mathrm{~s}$ ) in average.

In addition, Figure 5.8 depicts how the average $D_{M}$ and the average $L_{M}$ changes over the simulation time for $N_{4}$ under $S_{3}$, i.e. to demonstrate Oppline's dissemination efficiency through high number of devices dispersed in a sparse network. For all of the demonstrated setups, both $D_{M}$ and $L_{M}$ succeed a steady performance during the simulation runs. The fixed length of $Q$, which is 10 in our experiments, has an influence on these persistent results obtained throughout the network operation. 

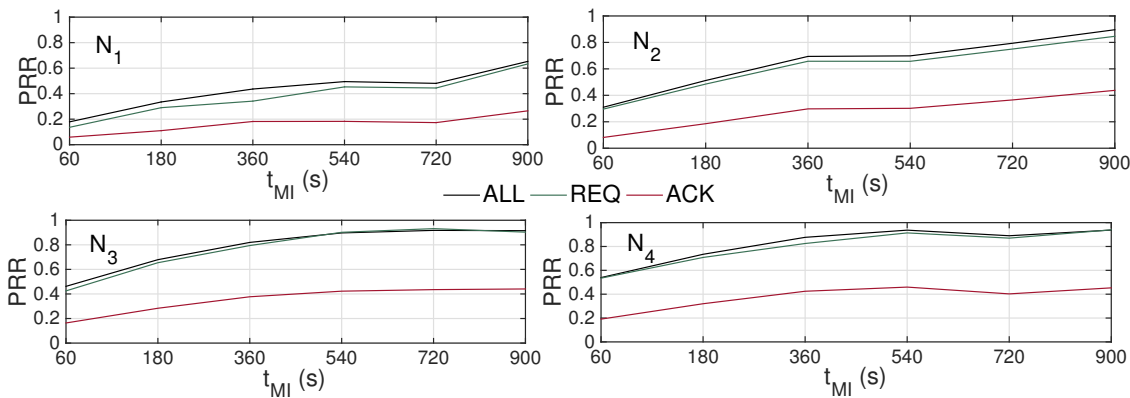

(a) $S_{1}$
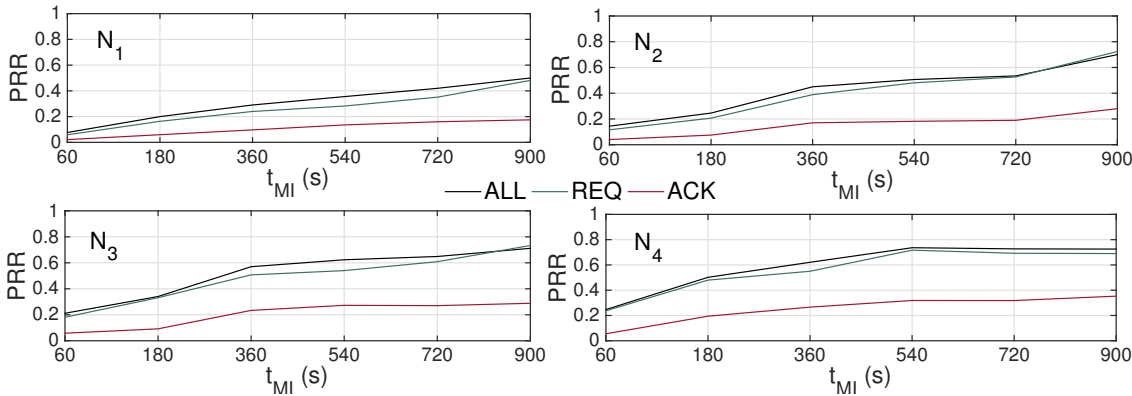

(b) $S_{2}$
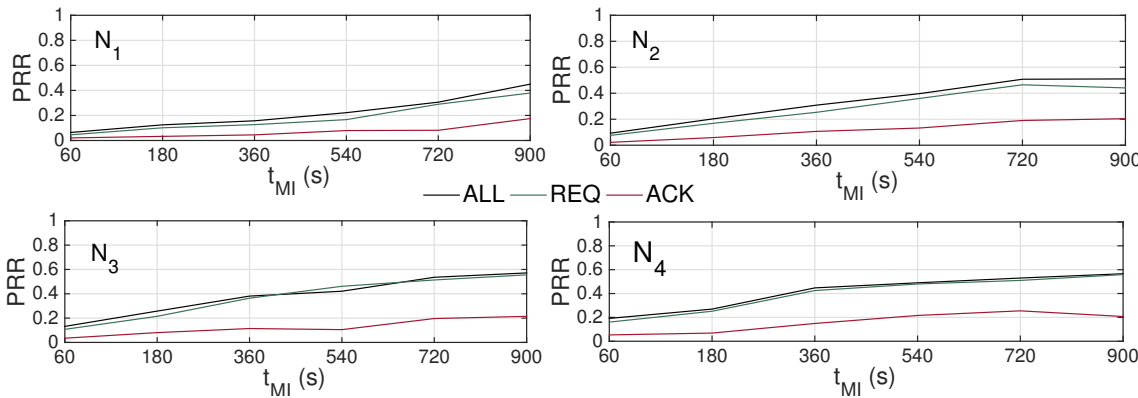

(c) $S_{3}$

Unicast data delivery performance 
FIGURE $5 \cdot 7$
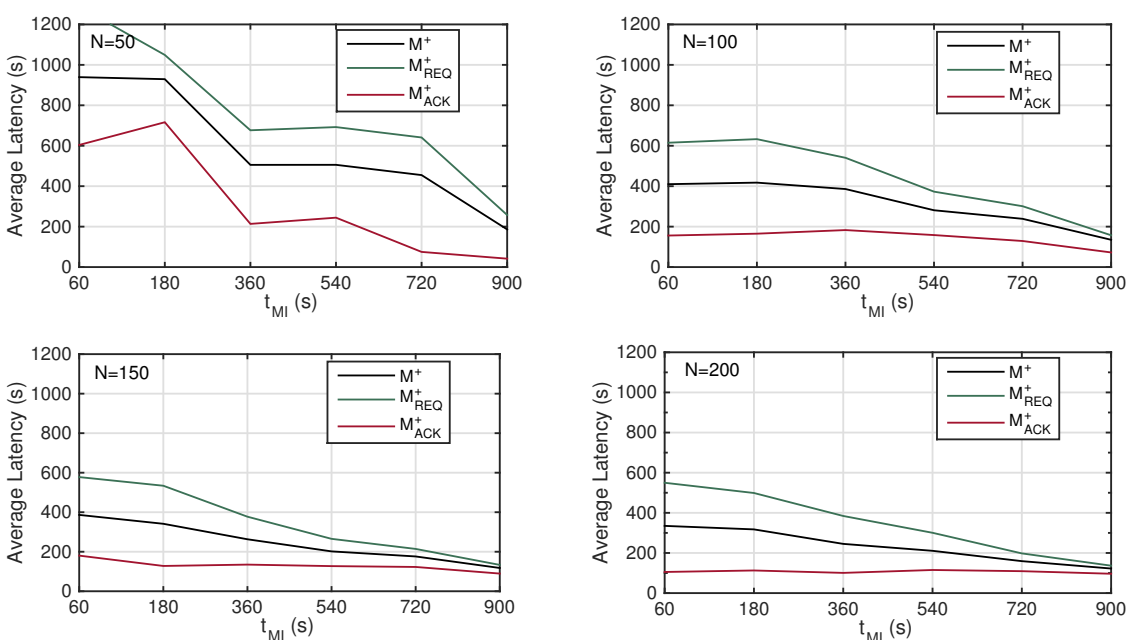

Unicast latency performance under $S_{1}$

FIGURE 5.8
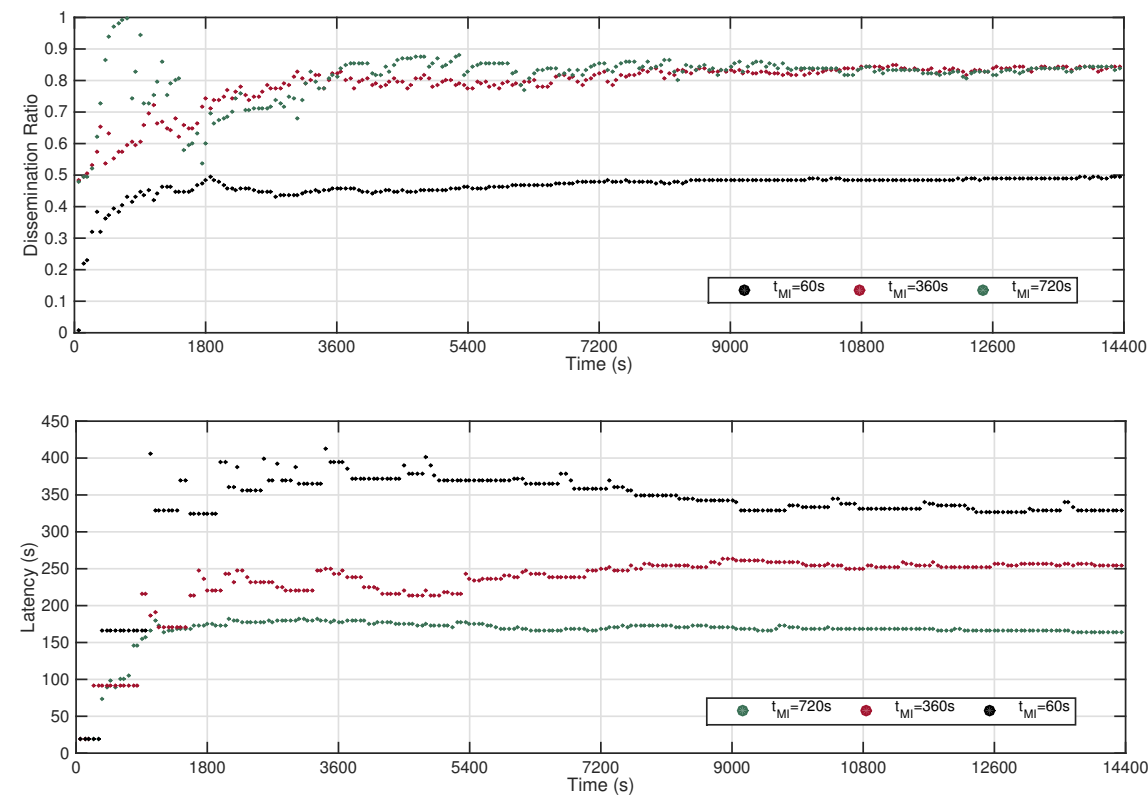

Data dissemination and latency over time with $N_{4}$ under $S_{3}$ 
The reason is that, newly-created messages which overwrite the oldest ones are not affected by message starvation even in the scenarios having high $M$. For instance, when $t_{M I}=60 \mathrm{~s}$, the network operation can still sustain a $D_{M}$ of $\approx 43 \%$ at 14400 s.

Similarly, the $L_{M}$ values reside between 300s and 400s. As the message creation interval increases, the dissemination performance increases. When $t_{M I}=360$ s, a $D_{M}$ of $\approx 80 \%$ can be achieved and a $L_{M}$ of $\approx 270$ s can be provided. The $D_{M}$ values obtained when $t_{M I}=720$ s are between the range of $\approx 80 \%$ and $\approx 85 \%$, but with lower $L_{M}$ values around $\approx 180$ s in average.

\subsubsection{Discussion}

The presented results signify three prominent outcomes:

i) Oppline achieves a high message delivery performance in dense deployments. As the densest setup, $N_{4}$ under $S_{1}$ gives an average PRR of $93 \%$ when $t_{M I}=900 \mathrm{~s}$. For the same network setup, the average PRR declines to $54 \%$ when $t_{M I}=60 \mathrm{~s}$. In terms of delivery latency, Oppline also maintains a high performance in dense deployments. The average $L$ values obtained in all tests fall in the range of 180s and 400s in average for $N_{2}, N_{3}, N_{4}$ under $S_{1}$.

ii) Oppline provides high suitability for low message frequency scenarios, or for the opportunistic ad hoc networks having single (or limited number of) message sources. Regardless of network density, the networking performance significantly increases as $t_{M I}$ is increased. Having the results from all network setups, the average PRR ranges between $50 \%$ and $95 \%$ when $t_{M I}=900 \mathrm{~s}$.

iii) Oppline can also be used in data dissemination scenarios. As the densest setup, $N_{4}$ under $S_{1}$ provides an average $D_{M}$ of $74 \%$ when $t_{M I}=60 \mathrm{~s}$ and an average $D_{M}$ of $91 \% t_{M I}=900 \mathrm{~s}$ throughout the tests.

Overall, Oppline is a lightweight opportunistic ad hoc routing and dissemination service with certain restrictions in its protocol design. According to our PRR $\mathrm{PCK}_{A C}$ results, the bidirectional routing of Oppline provides a moderate performance under reasonable network densities. Nonetheless, especially in crowded places, it can be a substantial alternative to the situated communication systems in the absence of an online network operation. PRR $R E Q$ results point out that Oppline can be an opportunistic line between group of friends as well as can be used to inform people in crowded places.

The design and implementation of Oppline is investigated with Wi-Fi beacons. However, the data exchange protocol of Oppline can be designed above any other wireless PHY/MAC protocol supporting beaconing. For instance, universal unique identifier (UUID) fields of BLE can be exploited in our protocol as well. Supporting any kind of wireless PHY/MAC, Oppline constitutes a good example for platform-independent and lightweight ad hoc short messaging services. 


\section{$5 \cdot 3$ BLESSED}

This section introduces BLESSED, a dual-radio OBN application running with the complementary utilization of the IEEE 802.11 and BLE standards. BLESSED stands for BLE profile and IEEE 802.11 Service Set Encoding-based Dissemination.

\section{5·3.1 Communication Model}

Data exchange between participating devices is enabled with the following wireless interfaces: Wi-Fi Hotspot mode, Wi-Fi Intrastructure mode, BLE Peripheral mode, and BLE Central mode.

In Wi-Fi Hotspot mode, devices advertise a previously encoded packet on their SSID fields. In Wi-Fi Infrastructure mode, devices perform scanning to discover the encoded packets within their wireless range. Since Wi-Fi Hotspot and Infrastructure modes are mutually exclusive, i.e. SSID advertising and scanning cannot run simultaneously, devices periodically switch between these modes. For coordination of SSID advertisements and switch times, devices further use BLE profiles, i.e. Universally Unique Identifier (UUID) fields. The BLESSED data exchange automaton has 3 superstates: i) $\mathrm{OB}, i i$ ) $\mathrm{BO}$, and iii) $\mathrm{U} \& \mathrm{~S}$.

Running the automaton with random initialization times, devices in an OppNet can be in either of these three superstates at a specific time. During the course of their OB services, denoted as $t_{O B}$, devices can only perceive $\mathrm{BOs}$ within their communication range. Likewise, during the course of their $\mathrm{BO}$ services, denoted as $t_{B O}$, devices can only perceive OBs within their communication range. On the other hand, during U\&S, denoted as $t_{U S}$, devices are unable to perceive messages over both communication channels. BLESSED utilizes a dynamic deadline optimization to reduce the number of same service conflicts (OB-OB and BO-BO).

\subsubsection{Service Scheduling}

Figure 5.9 demonstrates the concurrent operations during an $\mathrm{OB}$ or a $\mathrm{BO}$ service. A device constantly announces a packet $(P)$ with a fixed beacon interval $\left(t_{B I}\right)$. For $\mathrm{OB}, P$ is a preencoded SSID whereas for BO, a pre-encoded UUID. Both SSID and UUID conform specific encoding types consisting of three fields:

- Deadline: The designated end time of current service.

- Request: The flags to inform application-specific requests.

- Data: The contents of a message.

At each scan interval $\left(t_{S I}\right)$, a device decodes perceived Ps, i.e. announced deadlines, application-specific requests, and data of the nearby devices which run the opposite service. It stores the received data as well as the requests for further analysis at the next U\&S. Besides, it compares the decoded deadlines $\left(D_{i}\right)$ with its current deadline $\left(D_{\text {curr }}\right)$. As shown in Algorithm 3 , it adjusts its timer according to the device(s) having the earliest $D_{i}$. This adjustment ensures two advantages: First, it schedules its upcoming period with at least one device running in the opposite service. Second, it reduces redundant wait times for the current service. 


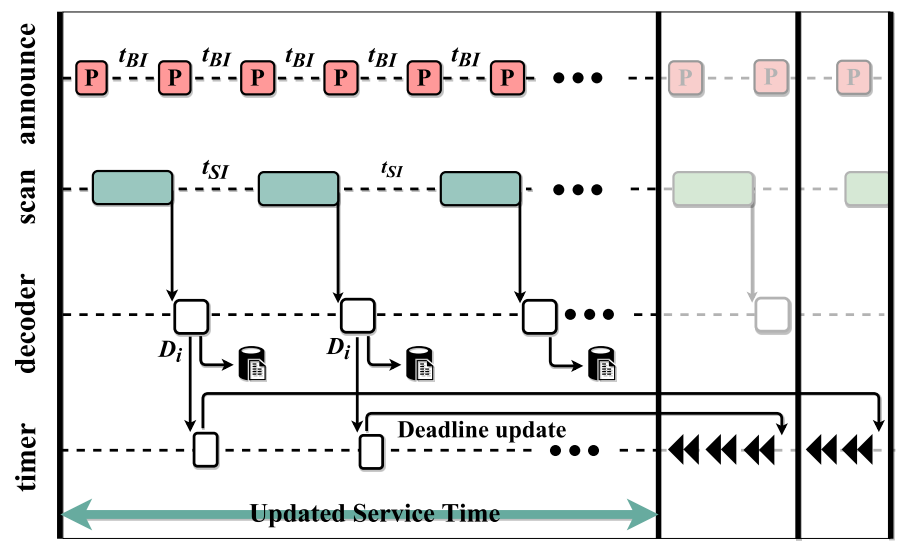

Sequence diagram of BLESSED operations

During decoding, a device uses a mode flag for the next U\&S. Once it detects at least one $P$ that has a period congruent to its period, mode is set to frequent. It is set to discovery if no $P$ is found. Algorithm 4 shows the use of mode for the determination of the next service and its corresponding deadline $\left(D_{\text {next }}\right)$ during U\&S. Frequent mode enables a device to periodically switch between the $\mathrm{OB}$ and $\mathrm{BO}$ services with a duration relatively shorter, one-half shorter in our design, than the actual service time.

Here, the aim is to increase the number of different $P$ advertisements and discoveries between the devices running the opposite services with the assumption that they will remain within range of each other. Besides, discovery mode assigns a random deadline, ranging from one-half shorter to one-half longer than the actual service time in our design. Here, the aim is to avoid OB-OB and BO-BO conflicts. Devices which cannot encounter any other device within their communication range extend their service times with the randomized deadlines.

Algorithm 3. Dynamic Deadline Determination

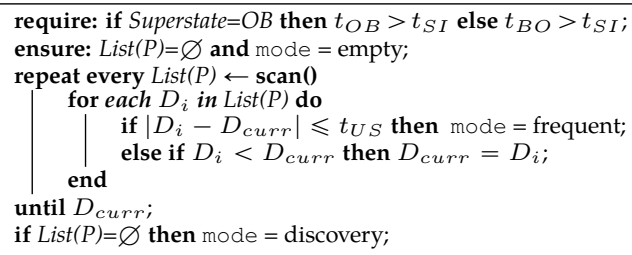

Algorithm 4. Next Service Determination

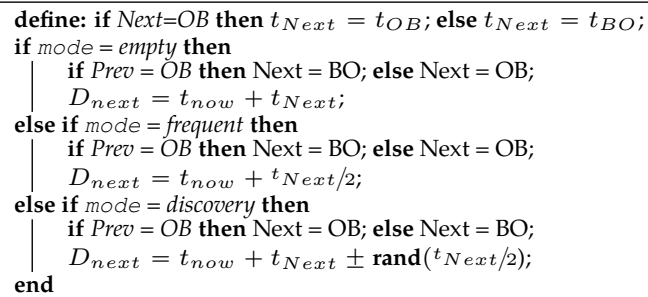




\subsubsection{Message Encoding}

IEEE 802.11 SSID and BLE UUID have editable lengths of only 32 ASCII bytes and 128 bits, respectively. Therefore, $P$ s have to be encoded succinctly. In our design, all $P$ structures consist of Deadline, Request, and Data fields.

Granted that all devices have the same current time, the last 3 digits of UNIX time are assigned to Deadline in our design. For SSID, Deadline is encoded to 2 ASCII bytes. For UUID, on the other hand, Deadline allocates 3 octets.

Request may involve a number of application-specific flags. Each mapped to a unique ASCII character, 94 different flags can be encoded on a single SSID byte. With a UUID bit, on the other hand, a single flag can be encoded. More detailed requests can be represented as the number of bytes/bits is increased. These flags are essential to increase dissemination efficiency. At every U\&S, Deadline and Data can differently be encoded based on the received flags at the previous superstate. In our design, Request is a single flag with two types, repeat and shift, allocating 1 ASCII byte on a SSID encoding and 1 bit on a UUID encoding. This flag serves as a notification to other devices for packet distribution, and has no effect on the service scheduling presented in Section 5.3.2.

For the remainder bytes/bits, Dat a may involve a chunk of messages, or a summarized message of a sensed phenomena. In SSID encoding, Dat a may contain additional message information such as source ID, creation time, event location, and intermediate router IDs. In UUID encoding, Dat a can only be used for primitive messages such as small integers and low precision values to represent a set of results or readings. To this respect, Data is used only on SSID encoding (OB), and is set to null on UUID encoding (BO) in our design.

\subsubsection{Data Exchange}

BLESSED represents two main restrictions for data exchange. First, Dat a forwarding is halfduplex, from an OB to BOs, at any given time. Second, network throughput is bound to the limited Data length that hinders sending all messages at once. Consequently, data dissemination is subject to potential delivery delays even under long-lasting contact durations. Nevertheless, BLESSED exploits the wireless broadcast advantage. At any given time, multiple OBs can be discovered by multiple BOs, and vice versa. This advantage provides Deadl ine and Request to better schedule the service deadlines and to better distribute several Data in an OppNet, respectively. As predefined variables in our design, $t_{O B}$ and $t_{B O}$ also play an important role on the data dissemination performance. Besides, Request is used to notify others of a current dissemination objective. Either serving as an $\mathrm{OB}$ or a $\mathrm{BO}$, once a device receives a Request of repeat, it selects its last received Data for advertisement at its next OB service. Here, the aim is to disseminate recent messages in an OppNet. This type of data dissemination can be useful for participatory monitoring applications. On the other hand, once a device receives a Request of shift, it sequentially selects one from all of its received Data for advertisement at each OB service. Here, the aim is to disseminate all of the messages with distributed chances in an OppNet. This type of data dissemination can be employed in opportunistic Twitter-like applications that allow participators to share less-critical short messages with each other. 


\section{Wi-Fi and BLE Coexistence}

Interference due to the collocation of $2.4 \mathrm{GHz}$ band Wi-Fi and Bluetooth is a fact for connectionoriented networks. However, OBN as a connection-free approach only uses advertisement beacon frames of these interfaces. As Figure 5.10 depicts, the BLE advertisement channels are non-overlapping with the Wi-Fi channels 1, 6, 11. BLE utilizes 3 advertisement channels $(37,38,39)$, 2MHz-wide, and they are non-overlapping with the Wi-Fi channels 1, 6, 11. In theory, Wi-Fi channels 1,6, 11 do not create an in-band interference with the BLE advertisement channels. To test their coexistence, BLE Peripheral and Wi-Fi Hotspot modes are run 100 times with different OB/BO orientations. OBs utilized the Wi-Fi Hotspot Channel 6 whereas BOs utilized random BLE advertisement channels. Given in Table 5.4, our packet reception rate (PRR) results prove that OBN is not affected by the coexistence of Wi-Fi and BLE.

\subsubsection{Networking Tests}

The networking performance of BLESSED is tested on a realistic event-based simulator which is fed with our applicability study results. Table 5.5 summarizes the properties of the designated networks as well as expresses the simulated parameters. The network setups consist of three different group densities $\left(N_{1}, N_{2}, N_{3}\right)$ each scattered over varying networking areas $\left(M_{1}-M_{5}\right)$. Device movements are simulated with the Random Waypoint mobility model. Considering the fact that $\mathrm{OB}$ and $\mathrm{BO}$ discoveries may not be reciprocal due to fluctuating signal powers, at different times, devices are given random radio ranges uniformly ranging from $25 \mathrm{~m}$ to $75 \mathrm{~m}$, and $20 \mathrm{~m}$ to $60 \mathrm{~m}$ for Wi-Fi and BLE interfaces, respectively. The results obtained in Section 3.3.3 are simulated for the wireless operations. The network setups are tested under different message frequencies and service operations.

All repeated 10 times, the tests are conducted regarding the communication model given in Section 5.3.1. The following results express the networking performance of the model in terms of data dissemination efficiency and point-to-point delivery efficiency.

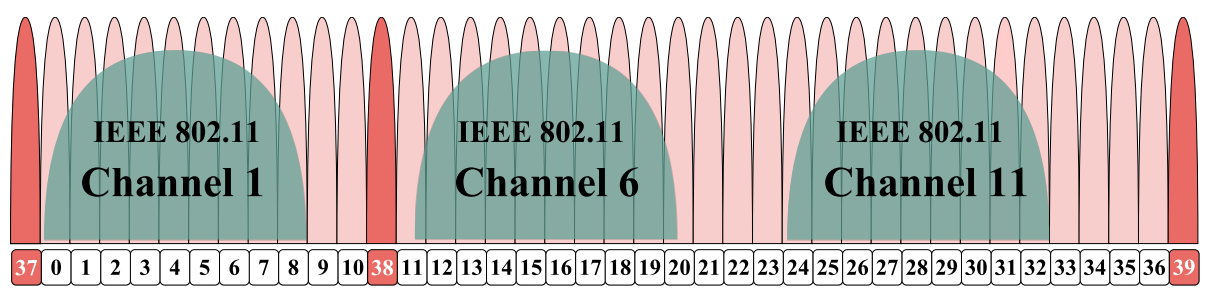

Non-overlapping advertisement channels

TABLE 5.4: SSID and UUID Reception Rates

\begin{tabular}{crrr}
\hline Orientation & UUID PRR & SSID PRR & \\
\hline 1 OB - 4 BO & $377 / 400$ & $98 / 100$ & \\
\hline 2 OB - 3 BO & $294 / 300$ & $193 / 200$ & \\
\hline 3 OB - 2 BO & $191 / 200$ & $286 / 300$ & \\
\hline 4 OB - 1 BO & $91 / 100$ & $391 / 400$ & \multirow{2}{*}{5 APPLICATIONS }
\end{tabular}


The service scheduling (Section 5.3.2) and the packet coordination (Section 5.3.3) functions are separately evaluated through all network setups and for all $t_{O B}=t_{B O}$. Besides, message creation interval $\left(t_{M I}\right)$ is set to 120 s for these tests.

Contrary to the unscheduled runs, the runs with Deadline optimization show a slight improvement on the data dissemination performance (Figure 5.11(a)). Especially in denser scenarios, the effect of the optimization is quite remarkable. In $M_{1}$, for instance, the data dissemination ratio is increased by $\approx 10 \%$ in average for all of the device groups. This improvement vanishes as the network setups get sparse.

There is no significant difference between Request: repeat and Request:shift in terms of data dissemination performance (Figure 5.11(b)). Nevertheless, Request : repeat performs much better than Request: shift in terms of point-to-point information delivery (Figure 5.12). At early times of the networking, it is evident that messages which are repeatedly advertised have higher delivery rates than alternatingly advertised messages. For both of these advertisement types, data delivery ratios run parallel as the network saturates in terms of number of messages (Figure 5.12(a)). But, repeatedly advertised messages are delivered almost twice earlier than alternatingly advertised messages (Figure 5.12(b)).

Regardless of network density or network size, too frequent U\&S initiations may negatively affect the networking performance, which is caused by the short $\mathrm{OB}$ and $\mathrm{BO}$ service time assignments $\left(t_{O B}\right.$ and $\left.t_{B O}\right)$ (Figure 5.13).

\subsubsection{Discussion}

The overall results indicate two prominent outcomes:

- BLESSED achieves high networking performance in dense deployments. As the densest setup, $N_{3}$ under $M_{1}$ gives an average data dissemination ratio of $71 \%$, with a standard deviation of $9 \%$, when $t_{M I}$ is $120 \mathrm{~s}$. For the same network setup, the average dissemination ratio decreases to $38 \%$, with a standard deviation of $5 \%$, when $t_{M I}$ is $30 \mathrm{~s}$.

- BLESSED provides high suitability for low message frequency scenarios, or for the OppNets having single (or limited) message sources. Regardless of network density, the networking performance significantly increases as $t_{M I}$ is increased (Figure 5.13). For $t_{M I}=180 \mathrm{~s}$, our model has reached a data dissemination ratio of $58 \%$, with a standard deviation of $7 \%$, as the average test results with all network setups.

TABle 5.5: Simulation Properties \& Parameters

\begin{tabular}{l}
\hline Test Period: $2 \mathrm{~h}$ for each combination of below parameters \\
\hline Number of devices: $N_{1}=50, N_{2}=100, N_{3}=150$ \\
\hline \begin{tabular}{lc} 
Maps $(m \times m):$ & $M_{1}=500 \times 500, M_{2}=1000 \times 1000, M_{3}=1500 \times 1500$ \\
& $M_{4}=2000 \times 2000, M_{5}=2500 \times 2500$ \\
\hline Mobility: Random Waypoint & Movement pause $(s):[0,300]$ \\
\hline Wi-Fi range $(m): 50 \pm[0,25]$ & BLE range $(m): 40 \pm[0,20]$ \\
\hline$t_{O B}=t_{B O}(s)=\{5,15,30\}$ & $t_{B I}, t_{S I}, t_{U S}:$ Table 3.1 \\
\hline Message creation interval $(s): t_{M I}=\{30,60,90,120,150,180\}$ \\
\hline$|Q|=10$
\end{tabular} \\
\hline
\end{tabular}




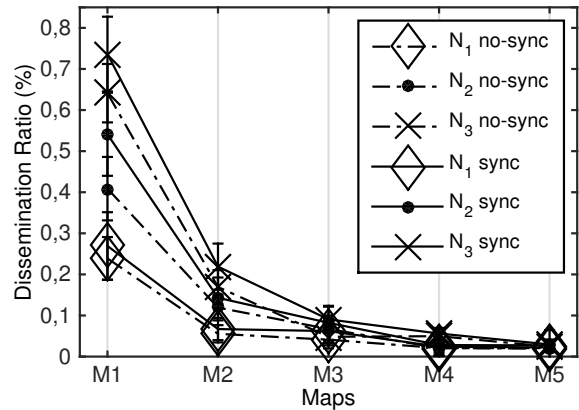

(a) Impact of scheduling on dissemination performance under several device densities

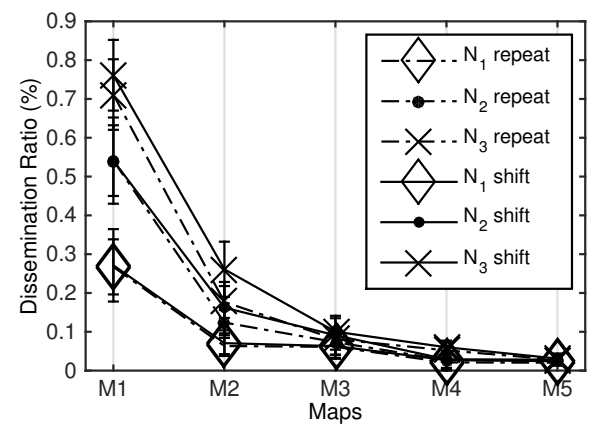

(b) Impact of packet coordination on dissemination performance under several device densities

FIGURE 5.12

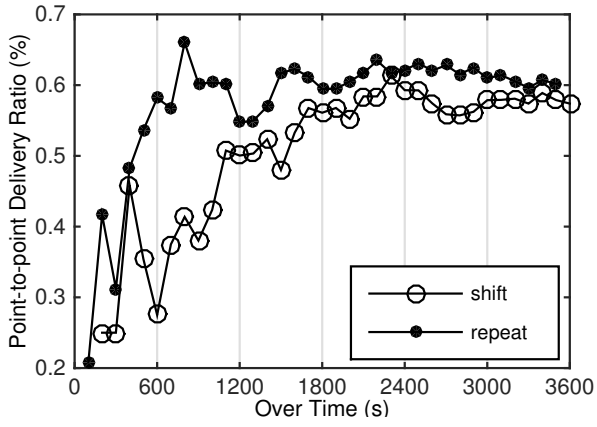

(a)

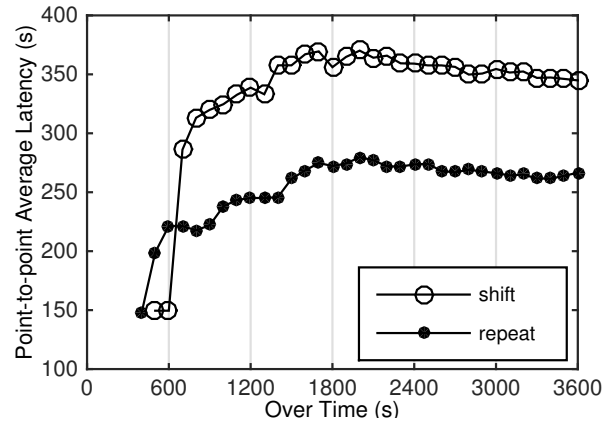

(b)

Packet Coordination: shift and repeat; the averaged results of the tests with $N_{1}, N_{2}, N_{3}$ under $M_{1} \cdot t_{M I}=120 \mathrm{~s}, t_{O B}=t_{B O}=30 \mathrm{~s}$

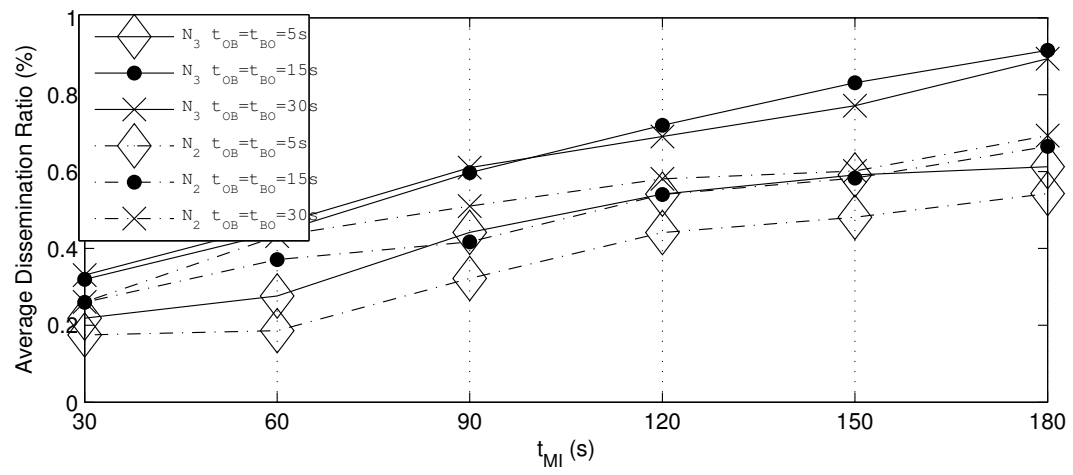

Impact of $t_{O B}=t_{B O}$ and $t_{M I}$ on dissemination performance; the averaged test results of $M_{1}$ 


\subsection{VADISS}

In this section, a collaborative way of intelligent traffic monitoring is offered with considerably larger coverage and less financial effort compared to the systems based on permanent infrastructures or dedicated on-board wireless adapters. Accordingly, an ad hoc opportunistic data dissemination protocol is introduced to enable participatory monitoring through smart mobile devices carried by traffic participants. Our protocol sets up open, free, and direct communication in vehicular environments with the ubiquitously present WiFi-compliant devices. It facilitates vehicular networking for any interested device without requiring special adaptations of the operating platforms, without violating the IEEE 802.11 standard, and without relying on any fixed road-side unit (RSU) installations.

Intelligent traffic systems are still demanding in terms of broad public reach. First and foremost, such systems have significantly high deployment costs [131]. An efficient routing runs on RSUs, or specialized adapters, or both [132]. Another issue is the standardization concerns for the PHY/MAC protocols . As the commonly accepted PHY/MAC standard, IEEE 802.11p is still open for enhancements together with IEEE 1609 protocol family. Above IEEE 802.11p, IEEE 1609 includes WAVE as a higher-level amendment [133]. Often tested with simulation models, both IEEE 802.11p and IEEE 1609/WAVE are offered with many modifications [134]. Real deployments might likely need specific hardware/software, hence seem to remain unsuited for end-use unless a stable set of standards becomes widespread.

Proposed as an upper-layer operation above IEEE 802.11, our protocol has a routing approach similar to IEEE 802.11p. As Figure 5.14 delineates, instead of IEEE 802.11 IBSS, IEEE 802.11p links exert a constant wildcard basic service set (WAVE BSS) in order to disable management frames related to association and authentication request/response [133]. Thus, links allow an association-free ad hoc mode to directly transmit and receive data frames via affiliated APs. By contrast, our protocol ignores Wi-Fi association, authentication, and connection establishment, and allows data exchange via the SSID of Wi-Fi beacon frames. Routed data is embedded in the SSID field and announced immediately to others sharing the same communication range. Regardless of smart mobile platform types, the routing therefore operates on top of the universal IEEE 802.11 standard. This highly-available opportunistic "link" can only be provided with at least one AP and one client serving in Wi-Fi Hotspot and Wi-Fi Infrastructure modes, respectively. To enable sending and receiving multiple data, the protocol employs a continuous switch between mutually-exclusive hotspot and client services.

Named VADISS (Vehicular Ad Hoc Dissemination via SSID), our protocol provides decentralized data sharing for many traffic monitoring applications such as, but are not limited to, road pavement monitoring, traffic density estimation, pollution detection. To this end, the effectiveness of VADISS is investigated through an online participatory road monitoring application. In a real-life deployment, several road anomalies are detected and the collected data is shared in a network setup. VADISS can be applied also for other PHY/MAC protocols as a specific use-case of Opportunistic Beacon Networks (OBN) presented in Chapter 4. It is a straightforward dissemination method compared to the protocols based on P2P, mesh, 

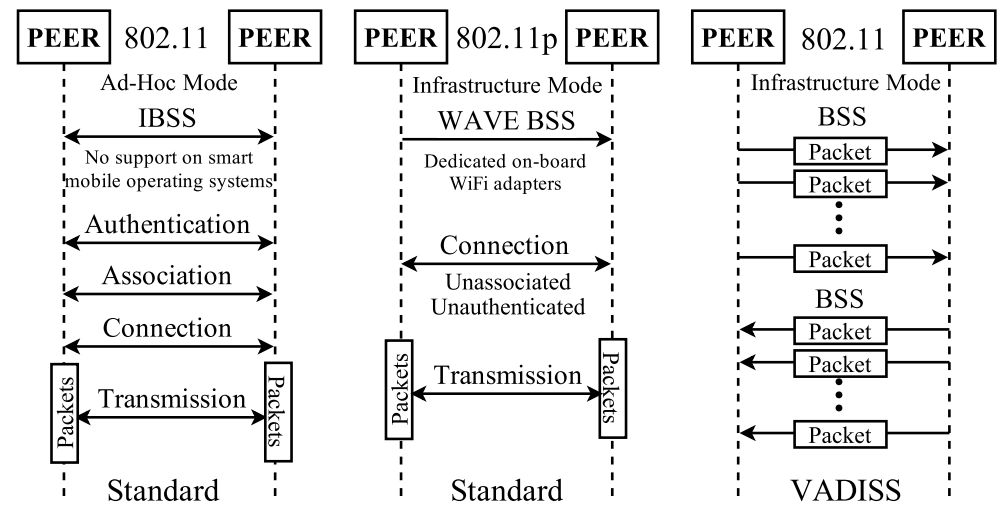

IEEE 802.11, IEEE 802.11p, and VADISS using OBN

and ad hoc connections which require either manual configurations or root privileges on today's mobile O/S firmwares [10]. It can operate directly on any platform, thus in networks of heterogeneous devices. Similar protocols have been recently studied on smart mobile platforms for data sharing $[29,52,135]$ and data dissemination $[68,69]$ applications, which mostly require special adaptations of the standards. Relevant to our approach, the study in [136] proposes a smartphone-based vehicular communication model via Wi-Fi interface. Nevertheless, the model necessitates internet access in order to reach collected traffic information through back-end servers. To the best of our knowledge, this study represents the first experimental study of utilizing wireless network identifiers as opportunistic information carriers for the sake of vehicular networking applications.

\subsubsection{Dissemination Protocol}

Our protocol is based on a low-throughput but connection-free message switching with an alternating use of Wi-Fi Infrastructure (client) and Wi-Fi Hotspot modes. In client mode, smart mobile devices used by traffic participants (hereafter, referred to as devices) can scan available $\mathrm{Wi}-\mathrm{Fi}$ APs in traffic environments. In hotspot mode, devices can serve as Wi-Fi APs with a specific SSID announcement. With today's technology, client and hotspot modes cannot simultaneously operate on a single Wi-Fi adapter. To make data exchange possible between devices, the automaton given in Chapter 3 is run with 3 states. One switches to OB state to announce a message, if any, as its current SSID. Serving in BO state, others scan to receive encoded SSIDs within the communication range. As long as there is at least one present message, the automaton cycles between States $\mathrm{OB}$ and $\mathrm{BO}$, thus devices periodically announce and can receive messages. If no message is available, State $O B$ is repeated. Based on received messages or application concerns, SSID can be encoded and $t_{O B}$ and $t_{B O}$ can be adjusted at each switch cycle. For instance, once a critical message is received, $t_{O B}$ can be increased to announce the message with a longer duration, or switches between States OB and BO can be made relatively infrequent with shorter $t_{B O}$. In Chapter 4 , this protocol is comprehensively analyzed with various parameters including $t_{O B}$ and $t_{B O}$. 
In spite of short inter-contact times in traffic environments, VADISS provides highly-scalable data dissemination, thanks to the high frequency in $t_{S I}$ and $t_{B I}$ provided by smart mobile platforms. Moreover, it exploits the wireless broadcast advantage since multiple SSID announcements can be received with a single scan operation. Nevertheless, it has low-throughput due to limited packet size and half-duplex operation. A packet can be at most 32 bytes-that is the maximum size allowed for the SSID field, and therefore is bound to contain limited data. Besides, it also introduces potential delays during message sharing since scan and announce operations are mutually exclusive. Consecutive switches between States OB and BO intermittently enable reception while blocking transmission, or vice versa, which may drop routing efficiency. Devices serving in same Wi-Fi mode cannot exchange data if their state switches are synchronized. In order to overcome this problem, a randomized short duration is added to $t_{O B}$ and $t_{B O}$ in each cycle.

\subsubsection{Participatory Road Traffic Monitoring}

Road anomalies such as potholes cause many traffic incidents ranging from congestions to accidents. Drivers en route tend to avoid such anomalies prudently or are unfortunately exposed to take whatever they may come across. In order to increase traffic-flow safety, participatory monitoring of road anomalies and unusual driver behavior requires a well-functioning networking between participants. To this end, VADISS is studied alongside with a smartphonebased online road anomaly and driver behavior detection application.

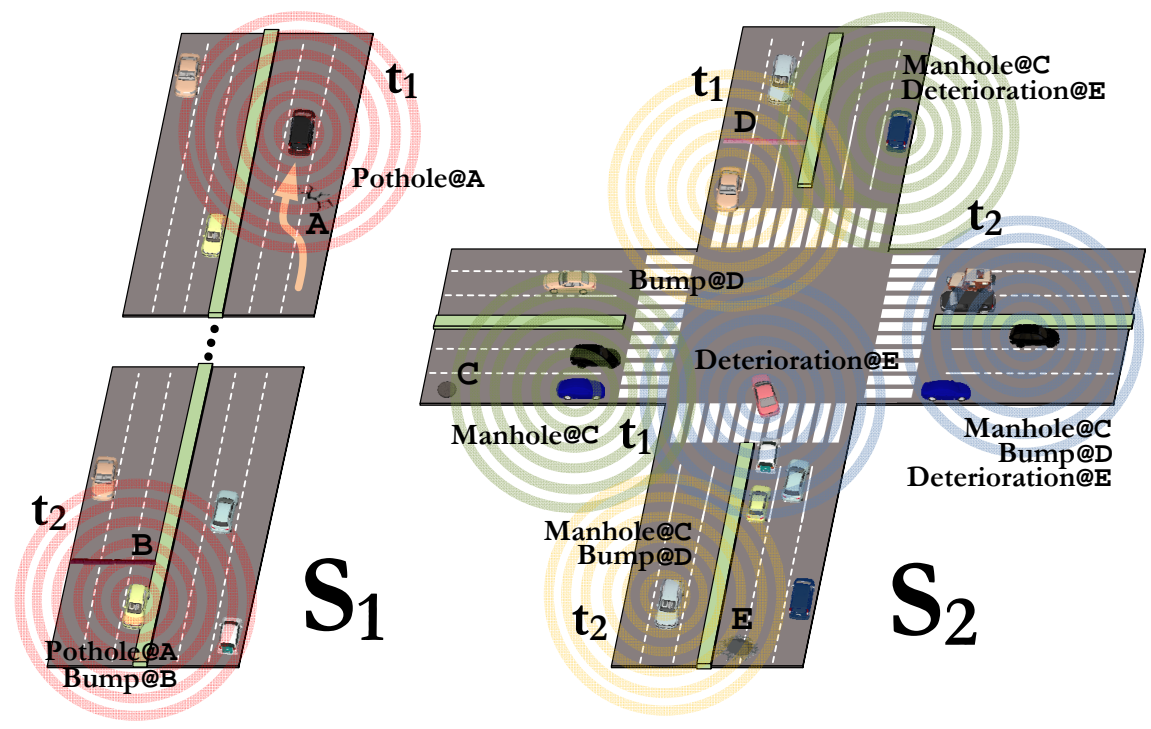

Smartphone-based Participatory Traffic Monitoring Scenarios 


\section{Information Dissemination Scenarios}

Figure 5.15 illustrates two general traffic information dissemination scenarios that engage smart devices of drivers forming an ad hoc opportunistic vehicular network through VADISS:

- $S_{1}$, alongside a two-way road.

- $S_{2}$, at an "at-grade" intersection of two or more roads.

Detecting all road anomalies in a single drive is unrealistic, hence the scenarios are based on increasing awareness about the road conditions and incidents by collaboration. Devices individually perform road monitoring and meanwhile opportunistically exchange road anomaly and driver behavior data. As shared data size increases in the network, the resulting information on traffic conditions gets actual and highly accurate.

\section{System Architecture}

Our application model together with VADISS represents a mobile cyber-physical system since devices cooperatively handle monitoring and data sharing over a network. Devices sense inertial changes with accelerometer, magnetometer, and gyroscope and locate the incidents with GPS sensors. For routing, devices intermittently utilize Wi-Fi client and hotspot modes. Figure 5.16 shows the sequence diagram of the system running road monitoring, data handling, and networking processes concurrently on a single device. The system employs two continuous processes: The first deals with road monitoring whereas the latter deals with data handling and networking.

Devices with the aforementioned specifications are used to detect and classify a set of road anomalies, driver behaviors, traffic streams in real-time. Initially, sensed data from the gyroscope, accelerometer, and magnetometer goes through a number of preprocessing steps including high-pass filtering, envelope calculation, demodulation, windowing, and wavelet transform. For online road anomaly detection and classification, the algorithm presented by Seraj et al. in [137] is run as a separate smartphone process.

In parallel, driver behavior results are clustered to perceive the reasons of unusual driver behavior. The clustering distinguishes accidental and periodic turns and swerves as well as to detect current traffic flow.

During our experiments, it is noticed that drivers tend to avoid the impact of an anomaly by swerving around it. The smartphone-based algorithm introduced in [138] is utilized to

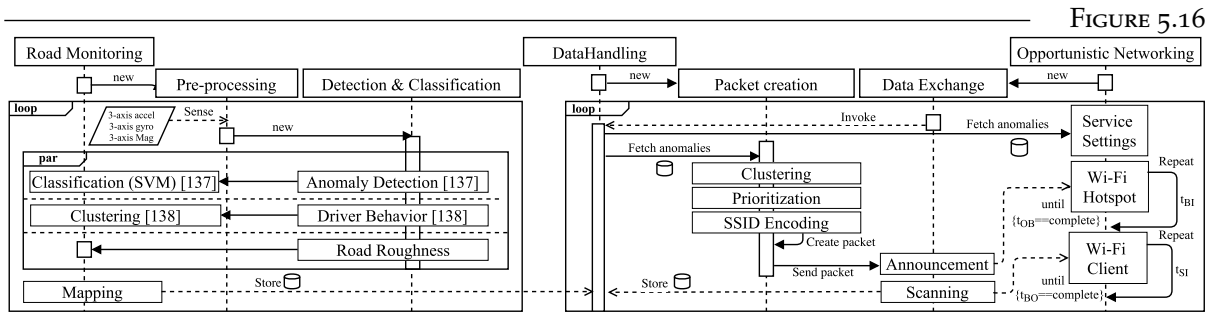

Sequence diagram of our Cyber-Physical Road Monitoring System 
detect this driver behavior. The algorithm detects all significant changes in direction of the vehicle and classifies them into several turn and swerve types. The aim of the algorithm is to increase the accuracy of detections by clustering the detected anomalies and swerves received by others in the network.

Another aspect of road monitoring is the road roughness determination. Studies show a strong correlation between vertical vehicle vibration and suspension frequency [139]. Thus, a current road roughness index can be estimated with the fourth level of decomposition on the vertical axis of the accelerometer. With participation, the index calculation is improved.

\subsubsection{Data Dissemination}

In this subsection, several definitions refer to the notations provided in Table 5.6 for the sake of brevity.

\section{Packet Creation}

To deal with the limited packet size, the following steps are held in each networking cycle:

i. Clustering: $a_{k} \in A$ which are detected in a radius less than $10 \mathrm{~m}$ are merged into one anomaly data.

ii. Prioritization: All $P_{i}$ are determined based on application concerns or current network status. $A$ is prioritized as $A^{\star}$.

iii. Packet Encoding: A packet, namely an encoded SSID is created based on two urgency sets, $U_{1}$ and $U_{2}$.

A packet is structured as a 32 byte case-sensitive ASCII string-the maximum length allowed for the SSID field. Hence, only a limited number of data in $A^{\star}$ is selected for the payload. The packet is encoded based on either Type I or Type II, as shown in Figure 5.17. Type I is selected if there are anomalies in $A^{\star}$ satisfying $U_{1}$. Otherwise, Type II is selected to embed the anomalies in $A^{\star}$ satisfying $U_{2}$. Type I contains at most $12\left(a^{\star}, \delta\right)$ pairs whereas Type II contains at most $4\left(a^{\star}, \ell\right)$ pairs.

TABLE 5.6: List of Notations

\begin{tabular}{ll}
\hline Symbol & Definition \\
\hline$A$ & Mapped road anomaly and driver behavior data set \\
\hline$A^{\star}$ & Prioritized $A$ with regard to a set of $P_{i}$ \\
\hline$P_{i}$ & A priority level for an anomaly type, where $i \leqslant n$ \\
\hline$n$ & Total number of anomaly types \\
\hline$a_{k}$ & A single anomaly data, where $a_{k} \in A$ and $k \in \mathbb{N}$ \\
\hline$a_{k}^{\star}$ & A single anomaly data, where $a_{k}^{\star} \in A^{\star}$ and $k \in \mathbb{N}$ \\
\hline$U_{1}$ & $\left\{a_{k}^{\star} \mid a_{k}^{\star}\right.$ happened in the last travelled kilometer $\}$, where $U_{1} \subseteq A^{\star}$ \\
\hline$U_{2}$ & $\left\{a_{k}^{\star} \mid a_{k}^{\star}\right.$ happened within a defined radius away $\}$, where $U_{2} \subseteq A^{\star}$ \\
\hline$\ell$ & A 6 ASCII bytes GPS position representation \\
\hline$\ell_{H}$ & Start position of the current OB service \\
\hline$\delta$ & Relative distance with regard to $\ell_{H}$ \\
\hline
\end{tabular}


TABLE 5.7: Mapping information

\begin{tabular}{|c|c|c|c|c|c|c|c|c|}
\hline Direction & $\mathrm{N}$ & $\mathrm{NE}$ & $\mathrm{E}$ & SE & $S$ & SW & $\mathrm{W}$ & NW \\
\hline Cycle (s) & 15 & 30 & 60 & 90 & & & & \\
\hline FS & Game & Fastest & & & & & & \\
\hline
\end{tabular}

Several ASCII mapping tables are used to compactly report the operational flags and anomaly data set. For numerical data, a decimal to ASCII conversion is applied. For conversion, 94 printable ASCII characters can be used, thus $\delta$ values less than 95 is indicated in 1 ASCII digit. Similarly, $\ell$ is encoded with 6 ASCII bytes, with special methods to encode latitude and longitude values shortly. A special mapping for $f_{1}$ is used to indicate the urgency type, or to inform an incomplete payload if there are not enough anomalies in $A^{\star}$. With the mapping in Table 5.7, $f_{2}$ is used to announce the direction, routing-related data, and current sensing properties. $f_{3}$ and $f_{4}$ are used for road roughness results. Based on the used ASCII mappings, received packets are decoded and stored locally.

\section{Data Exchange}

At each switch cycle, a ready packet is announced in State $\mathrm{OB}$, after which the traffic is scanned in State BO. Once new data are detected or received, $A$ is reprioritized. Just before the packet creation, the operational flags, the encoded anomaly data set, and other related numerical data are updated based on the start time of State OB and $\ell_{H}$.

FIGURE 5.17

\section{Type I}

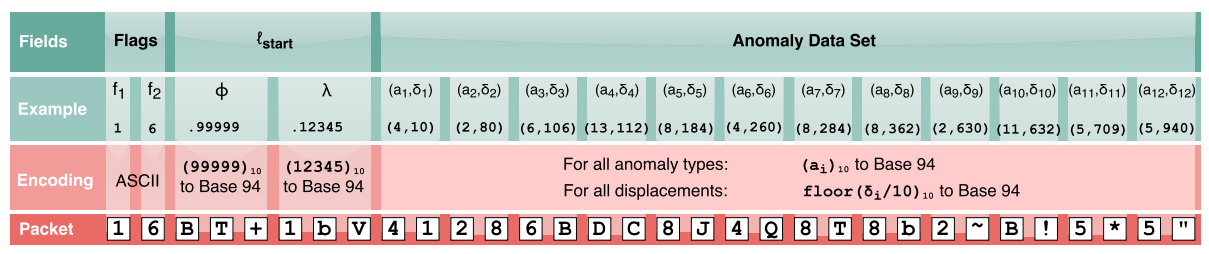

\section{Type II}

\begin{tabular}{|c|c|c|c|c|c|c|c|c|c|c|c|c|c|c|c|}
\hline \multirow{3}{*}{$\begin{array}{l}\text { Fields } \\
\text { Example } \\
\text { Encoding }\end{array}$} & \multirow{2}{*}{\multicolumn{3}{|c|}{ 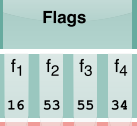 }} & \multicolumn{12}{|c|}{ Anomaly Data Set } \\
\hline & & & & \multicolumn{3}{|c|}{$\begin{array}{c}\left(a_{1}, l_{1}\right) \\
(4,[.01234, .99999])\end{array}$} & \multicolumn{3}{|c|}{$\begin{array}{c}\left(a_{2}, \ell_{2}\right) \\
(2,[.12345, .98765])\end{array}$} & \multicolumn{3}{|c|}{$\begin{array}{c}\left(a_{3}, l_{3}\right) \\
(6,[.34755, .55035])\end{array}$} & \multicolumn{3}{|c|}{$\begin{array}{c}\left(a_{4}, l_{4}\right) \\
(13,[.19519,23420])\end{array}$} \\
\hline & & ASCII & & & $\begin{array}{l}(01234)_{10} \\
\text { to Base } 94\end{array}$ & $\begin{array}{l}(99999)_{10} \\
\text { to Base } 94\end{array}$ & & $\begin{array}{l}(12345)_{10} \\
\text { to Base } 94\end{array}$ & $\begin{array}{l}(98765)_{10} \\
\text { to Base } 94\end{array}$ & & $\begin{array}{l}(34755)_{10} \\
\text { to Base } 94\end{array}$ & $\begin{array}{l}(55035)_{10} \\
\text { to Base } 94\end{array}$ & & $\begin{array}{l}(19519)_{10} \\
\text { to Base } 94\end{array}$ & $\begin{array}{l}(23420)_{10} \\
\text { to Base } 94\end{array}$ \\
\hline Packet & $\mathrm{G}$ & $r$ & t $Y$ & 4 & $\begin{array}{lll}0 & D \\
\end{array}$ & $\mathrm{~B}, \mathrm{~T}+$ & 2 & $\begin{array}{lll} & b & \\
\end{array}$ & \begin{tabular}{|l|l}
$B$ & G
\end{tabular} & 6 & \begin{tabular}{|l|l|l}
3 &. & $\wedge$ \\
\end{tabular} & $\begin{array}{lll}6 & \mathrm{~L} \\
\end{array}$ & $D$ & \begin{tabular}{|ll} 
& $\mathrm{z}$ \\
\end{tabular} & \begin{tabular}{|l|l|}
2 & \\
\end{tabular} \\
\hline
\end{tabular}

Packet Encoding Types. Type I allocates 2 bytes for two operational flags, 6 bytes for the GPS $\left(\ell_{H}\right)$, and the rest for the anomaly data set. Type II allocates 4 bytes for four the flags, and the rest for the anomaly data set. 


\subsubsection{Performance Analysis}

The data dissemination performance is tested with 10 Samsung S4 Mini phones, denoted as $D_{1} \cdots D_{10}$. The phones are placed 5 by 5 in 2 cars, $V_{1}$ and $V_{2}$. Table 5.8 gives our experimental setup. In opposite directions, $V_{1}$ and $V_{2}$ shuttle between two base stations for 9 times, each time with a different velocity $(v)$ and $t_{B O}=t_{O B} . V_{1}$ and $V_{2}$ wait at their bases for a time, then start travelling at the same time, and meet in the halfway. In the beginning of each shuttle, all $D_{i}$ create a unique network packet, denoted as $p_{1}^{c} \cdots p_{5}^{c} \in V_{1}$ and $p_{1}^{c} \cdots p_{5}^{c} \in V_{2}$, at random times. The average data dissemination rate solely in the opposite direction $\left(s_{O}\right)$ is calculated with the general formula given in Equation 5.1. Belonging to either $V_{1}$ or $V_{2}$, a $p_{i}^{r}$ denotes a received packet, respectively. The average dissemination rate in both directions $\left(s_{B}\right)$ is calculated with Equation 5.2. Since $D_{1} \cdots D_{5} \in V_{1}$ and $D_{1} \cdots D_{5} \in V_{2}, n=5$ in our example.

$$
\begin{gathered}
s_{O}=\left(\frac{\sum_{i=1}^{n} p_{i}^{r} \in V_{2}}{\sum_{i=1}^{n} p_{i}^{c} \in V_{1}}+\frac{\sum_{i=1}^{n} p_{i}^{r} \in V_{1}}{\sum_{i=1}^{n} p_{i}^{c} \in V_{2}}\right) /\left(p^{c} \in V_{1}+p^{c} \in V_{2}\right) \\
s_{B}=\left(\frac{\sum_{i=1}^{n} p_{i}^{r} \in V_{1}+\sum_{i=1}^{n} p_{i}^{r} \in V_{2}}{\sum_{i=1}^{n} p_{i}^{c} \in V_{1}+\sum_{i=1}^{n} p_{i}^{c} \in V_{2}-1}\right) /\left(p^{c} \in V_{1}+p^{c} \in V_{2}\right)
\end{gathered}
$$

Figure 5.18(a) illustrates the data dissemination results with regard to different $t_{B O}=t_{O B}$ and relative speed $\left(v_{r}=2 \times v\right)$. The blue bars show $s_{O}$ results whereas the white bars depict $s_{B}$ results stacked on top of $s_{O}$ results. The overall results show that $t_{B O}=t_{O B}$ and $v_{r}$ have a slight influence on the data exchange performance. The average rate varies between $20 \%$ and $30 \%$ for the dissemination in opposite directions. The overall rate increases up to $48 \%$ with every-which-way dissemination.

In order to test the robustness of VADISS in broader vehicular environments, scaled-up tests are taken with our simulator Oppliqué presented in Appendix B. Table 5.9 expresses the deployments based on the scenarios $S_{1}$ and $S_{2}$ described in Section 5.4.2. For $S_{1}$ and $S_{2}$, a unique packet is created per $\mathrm{km}$ travelled in each device. Thus, the average number of packets created per vehicle in $S_{1}$ is $\approx 10$, and in $S_{2}$ is $\approx 20$. Figure 5.18 (b) depicts the data dissemination results with regard to different number of vehicles and under different traffic stream scenarios. The color bars show dissemination rates obtained in $S_{1}$ whereas the white bars that of in $S_{2}$.

TABle 5.8: Field Test Parameters

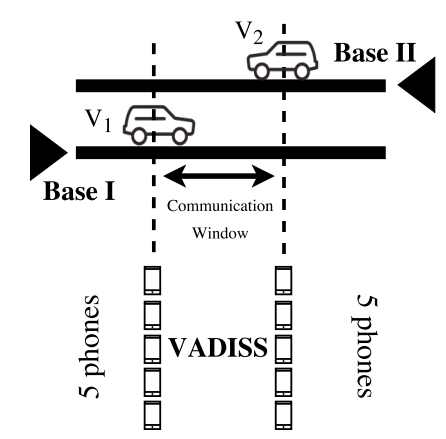

Scenario: 600m-long two-lane expressway

Shuttle 1: $v=40 \mathrm{~km} / \mathrm{h}, t_{B O}=t_{O B}=5 \mathrm{~s}$

Shuttle 2: $v=50 \mathrm{~km} / \mathrm{h}, t_{B O}=t_{O B}=5 \mathrm{~s}$

Shuttle 3: $v=60 \mathrm{~km} / \mathrm{h}, t_{B O}=t_{O B}=5 \mathrm{~s}$

Shuttle 4: $v=40 \mathrm{~km} / \mathrm{h}, t_{B O}=t_{O B}=15 \mathrm{~s}$

Shuttle 5: $v=50 \mathrm{~km} / \mathrm{h}, t_{B O}=t_{O B}=15 \mathrm{~s}$

Shuttle 6: $v=60 \mathrm{~km} / \mathrm{h}, t_{B O}=t_{O B}=15 \mathrm{~s}$

Shuttle 7: $v=40 \mathrm{~km} / \mathrm{h}, t_{B O}=t_{O B}=30 \mathrm{~s}$

Shuttle 8: $v=50 \mathrm{~km} / \mathrm{h}, t_{B O}=t_{O B}=30 \mathrm{~s}$

Shuttle 9: $v=60 \mathrm{~km} / \mathrm{h}, t_{B O}=t_{O B}=30 \mathrm{~s}$

Other parameters: $t_{B I}=100 \mathrm{~ms}, t_{S I}=100 \mathrm{~ms}$ 


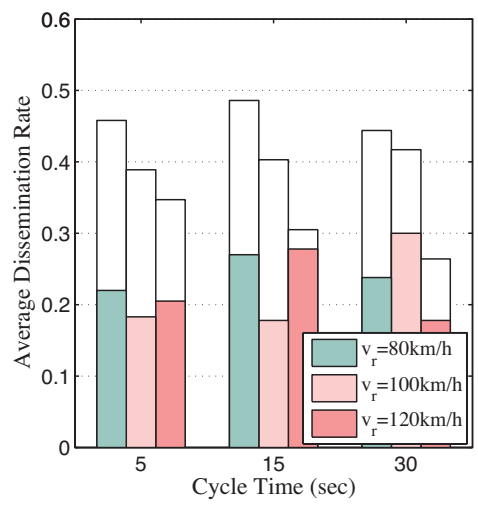

(a) Field Test

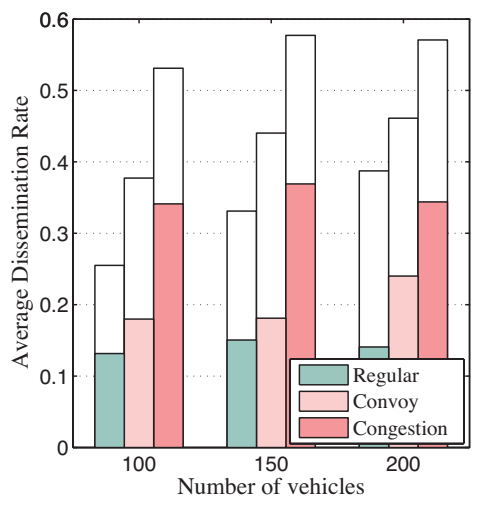

(b) Simulation

Opportunistic Networking Results

In $S_{1}$, during regular traffic flow, the average data dissemination rate is $12 \%$ regardless of vehicle density. As the vehicles move coherently (convoy), the performance slightly increases. In congested traffic, this rate increases up to $35 \%$ in average. In $S_{2}$, the performance drastically increases since the vehicles have more inter-contact times. In average, the dissemination rate is $32 \%, 42 \%$, and $54 \%$ for regular, convoy, and congestion, respectively.

The networking tests represent the average packet switching performance. Nonetheless, network packets are composed of several anomaly data set as previously presented. To this end, VADISS results are evaluated with the online participatory monitoring performance results as well. First, the road anomaly detection algorithm presented in Section 5.4.2 is tested on the Dutch and Albanian roads. Putting our algorithm into a context of comparison, an objective audiovisual method is used to evaluate our results. Table 5.10 shows the number of anomalies detected, classified, and evaluated for each trip. Anomalies are classified into 3 types: severe, mild, and span. Overall, a consistent accuracy of $\approx 90 \%$ is obtained regardless of vehicle and road types.

TABLE 5.9: Simulation Parameters

Real Map-based Movement with paths

\begin{tabular}{ll}
\hline \multicolumn{3}{l}{ Number of vehicles (devices): $100,150,200$} \\
\hline \multicolumn{3}{l}{ Vehicle speed: [40...90]km/h, comm.range $=90 \mathrm{~m}$} \\
\hline $\mathrm{S}_{1}$-Regular Average Wait Time: & $10 \mathrm{~s}$ \\
\hline $\mathrm{S}_{1}$-Convoy Average Wait Time: & $20 \mathrm{~s}$ \\
\hline $\mathrm{S}_{1}$-Congested Average Wait Time: & $30 \mathrm{~s}$ \\
\hline $\mathrm{S}_{2}$-Regular Average Wait Time: & $30 \mathrm{~s}$ \\
\hline $\mathrm{S}_{2}$-Convoy Average Wait Time: & $60 \mathrm{~s}$ \\
\hline $\mathrm{S}_{2}$-Congested Average Wait Time: & $90 \mathrm{~s}$ \\
\hline$t_{B O}=t_{O B}=15 \mathrm{~s}, 30 \mathrm{~s}, t_{B I}=100 \mathrm{~ms}, t_{S I}=100 \mathrm{~ms}$, \\
$t_{X O B}=4.2 \pm[0.1 .7] \mathrm{s}, t_{X B O}=2.8 \pm[0.1 .3] \mathrm{s}$
\end{tabular}

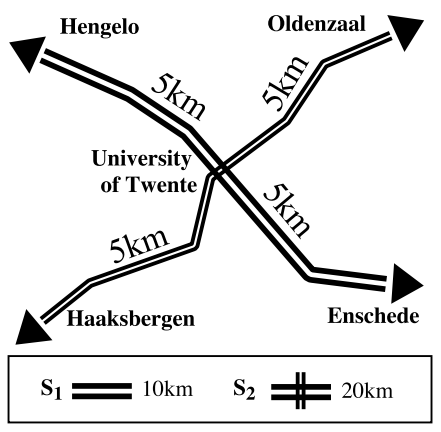


Based on the online road anomaly detection test results, Table 5.11 gives the average number of severe anomalies detected in each trip and their estimated dissemination rate in our simulation scenarios. Results evidently demonstrate that having less number of anomalies provides high dissemination rate. Trips 1 and 2 result in better performance compared to Trips 3-5 as the packets can announce an anomaly longer as long as it is overwritten with new anomalies. For regular traffic stream scenarios, the maximum dissemination rate reached is $17 \%$ whereas for other scenarios it is $73 \%$. Oppositely, having high number of anomalies has a negative effect on the dissemination performance, especially for the scenarios that devices have short inter-contact durations. In Trip 5, for instance, $S_{1}$-regular can provide a dissemination rate only up to $13 \%$. Nevertheless, $S_{1}$-congestion shows $37 \%$ of dissemination rate. This amount goes up to $56 \%$ for the scenarios in which the vehicles move more coherently.

\subsubsection{Discussion}

VADISS has been introduced together with a smart-phone based online participatory road monitoring applicaton. A set of real-traffic experiments has been held to investigate the data exchange efficiency. The results have indicated the fast and high dissemination performance of VADISS in several scenarios. In average, $38 \%$ of dissemination rate of critical traffic monitoring data has been reached. While the wireless broadcast advantage of VADISS is exploited, $\approx 63 \%$ of dissemination rate has been reached in denser scenarios.

The beacon format presented for VADISS can be also designed differently for other applications related to either safety or non-safety purposes in vehicular environments. Safety applications include information dissemination regarding hazardous situations such as warning of accidents, emergency vehicles approaching, alarming driver behavior [140], and so forth. Non-safety applications include infotainment such as monitoring information about parking availability, sharing road maps and local commerce information, and so forth.

TABLE 5.10: Road anomaly classification results

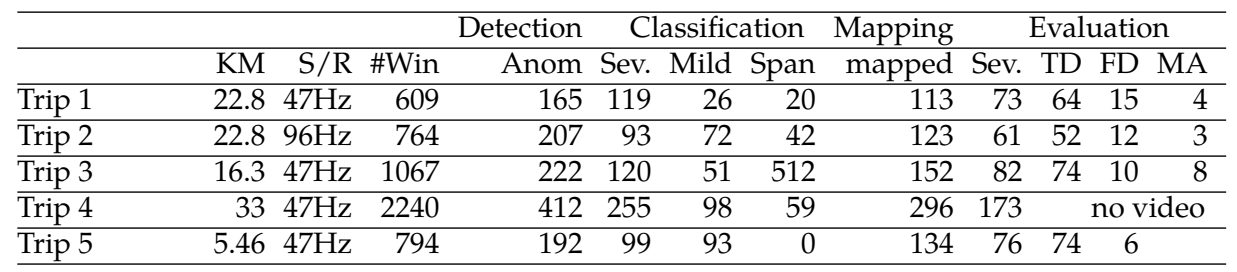

Sev. $=$ Severe, $\mathrm{TD}=$ True Detections, $\mathrm{FD}=$ False Detections, MA= Missed Anomalies, \#Win= Number of windows

TABLE 5.11: Road anomaly dissemination results

\begin{tabular}{lrrrrrrrrr}
\hline & Mapping & Anomaly & \multicolumn{1}{c}{ Packet } & \multicolumn{6}{c}{ Estimated Dissemination Rate } \\
\hline & per km & per veh. & per dev. & S1-reg & S1-cvy & S1-cng & S2-reg & S2-cvy & S2-cng \\
\hline Trip 1 & 3.20 & 32 & 3 & 0.15 & 0.24 & 0.41 & 0.36 & 0.47 & 0.61 \\
\hline Trip 2 & 2.67 & 27 & 3 & 0.17 & 0.28 & 0.49 & 0.43 & 0.57 & 0.73 \\
\hline Trip 3 & 5.03 & 51 & 5 & 0.15 & 0.25 & 0.43 & 0.38 & 0.50 & 0.64 \\
\hline Trip 4 & 5.24 & 53 & 5 & 0.15 & 0.24 & 0.41 & 0.37 & 0.48 & 0.62 \\
\hline Trip 5 & 13.92 & 140 & 12 & 0.13 & 0.22 & 0.37 & 0.33 & 0.44 & 0.56 \\
\hline
\end{tabular}




\subsection{Concluding Remarks}

This chapter has proposed three applications which inherit the OBN concept. These applications are represented as to be used as specialized opportunistic short message services for data dissemination and data routing purposes. Their target scenarios cover different areas in social space ranging from small-scale to large-scale networking environments.

Section 5.2 has presented Oppline which supports bi-directional and multi-hop short messaging between peers in crowded events such as concerts and sports organizations. The performance evaluation of Oppline has been conducted over a large set of simulations validated with a real-world deployment. For the real-world tests, Oppline is developed as an Android application and its networking is studied with 20 smartphones. The simulations are conducted to study Oppline under larger setups. It has been shown that Oppline achieves a promising delivery performance in diverse point-to-point scenarios under reasonable device density.

Section 5.3 has presented BLESSED which is an enhanced version of OBN with dual-radio utilization. The simulation results verify the benefits of complementing beaconing of one radio with scanning of another radio in terms of data delivery, data dissemination, and delay. Furthermore, it has been shown that the scheduling presented for BLESSED outperformed the classic OBN approach in diverse network densities.

Section 5.4 has presented VADISS which is primarily intended and tested for highway scenarios. It has been shown that OBN is suitable for rapid dissemination of critical traffic information with smart mobile devices. It has also been shown that multiple road monitoring data can be encoded in one beacon frame, and this improves the efficiency in delivering relevant information to interested traffic participants.

Based on the results presented in this chapter, it can be concluded that OBN can serve as an efficient multi-purpose protocol in any type of urban scenarios. Ranging from small-scale to large-scale networks, OBN is suitable for both point-to-point short messaging and data dissemination. From the performance evaluation outcomes, it is obvious that OBN considerably takes advantage of network density. As the sparsity increases, it is imperative to support the operation of the applications with infrastructures. The applications are evaluated solely on the case of decentralized OppNet setups. However, a fixed or a mobile backbone such as cellular base stations, APs, and RSUs will certainly play a role in improving connectedness inside the OppNet.

OBN incorporates specialized message formats in these applications. All of these applications can be used for different purposes in different scenarios by providing them with different beacon encodings. A message format is mostly of interest to a number of users in shared local OppNet regions that can be one-hop to many hops. In any case, the OBN-based applications can provide high scalability and low transmission delay in such purposes, moreover, can be used to fill the global Internet gaps in those regions. Thanks to the Cocoon architecture, it is also possible to use a multitude of these applications at the same time within an OppNet. 



\section{CHAPTER 6}

\section{Conclusions \& Future Prospects}

THIS THESIS HAS INTRODUCED COCOON, A NOVEL ARCHITECTURE DEVISED FOR SMART MOBILE PLATFORMS TO SUPPORT THE DEVELOPMENT AND OPERATION OF OPPORTUNISTIC COMMUNICATION APPLICATIONS AND SERVICES ENVISIONED FOR SOCIAL USE. COCOON INTEGRATES VERSATILE AND LIGHTWEIGHT OPPORTUNISTIC COMMUNICATION METHODS WITH A NEW COLLECTION OF APPLICATIONS THAT ARE FREELY ACCESSIBLE BY EVERYONE USING SMART MOBILE DEVICES. THE PRESENTED APPLICATIONS COVER A WIDE VARIETY OF APPLICATION AREAS, INCLUDING SHORT MESSAGE SERVICES IN CHALLENGED ENVIRONMENTS, SAFETY MONITORING IN VEHICULAR ENVIRONMENTS, AND DATA DISSEMINATION IN SEVERAL DEMANDING SCENARIOS. IN ORDER TO BRING THOSE APPLICATIONS INTO REALITY, A PRACTICAL NETWORK DESIGN IS DEVELOPED THAT CAN ADDRESS THE CRITICAL AND PECULIAR CHALLENGES OF OPPNETS. THE NETWORK DESIGN OF COCOON IS GENERIC IN TERMS OF IMPLEMENTABILITY ON TOP OF ANY KIND OF WIRELESS NETWORKING STANDARD, i.e. IT PROVIDES A BASIS TO OVERCOME THE CONNECTIVITY LIMITATIONS AND RESTRICTIONS REFLECTED BY TODAY'S MOBILE OPERATING SYSTEM PLATFORMS. THE CONNECTIVITY SCHEME OF COCOON IS ACHIEVED WITH A NOVEL CONCEPT CALLED OPPORTUNISTIC BEACONS THAT ALLOWS DEVICES TO SHARE INFORMATION BETWEEN EACH OTHER WITHOUT A CONNECTION. THE NETWORKING PERFORMANCE OF OPPORTUNISTIC BEACONS IS STUDIED OVER SEVERAL NETWORK SETUPS AS WELL AS WITH A COMPREHENSIVE SET OF REAL-WORLD EXPERIMENTS AND VERIFIED SIMULATIONS.

THIS CHAPTER OUTLINES THE CONTRIBUTIONS PRESENTED IN THIS THESIS, DRAWS CONCLUSIONS ABOUT THE WORKS STUDIED, ELABORATES ON THE FUTURE RESEARCH DIRECTIONS, AND CONCLUDES WITH BRIEF REMARKS REGARDING PROSPECTIVE STUDIES IN THE FIELD OF OPPORTUNISTIC COMMUNICATIONS. 


\subsection{ReFLECTIONS \& IMPLICATIONS}

This section reflects upon the research presented in this thesis and presents implications for the OppNet research community. First, the thesis contributions are recapitulated with regard to the main research question of the thesis. Second, the answers to the research questions are reflected. Third, the lessons learned are presented.

\subsubsection{Contributions Revisited}

The main focus of this thesis is on the design, development, and analysis of Cocoon, our community-oriented context-aware opportunistic networking architecture. Cocoon employs lightweight and versatile opportunistic connectivity schemes that facilitate universal, rapid, and energy-efficient information sharing between smart mobile devices without requiring sophisticated configurations. In addition to Cocoon, an opportunistic data forwarding and dissemination model called Opportunistic Beacon Networking (OBN) is presented. The OBN model is experimented in real-world setups and validated simulations in different OppNet application scenarios. The soundness of the model in terms of networking performance is shown. To this respect, the overarching research question addressed in this thesis is:

How can opportunistic ad hoc communications be achieved with smart mobile devices in a practical, efficient, and generic manner while fulfilling specific requirements of different applications developed for specialized environments?

The following contributions are presented in the thesis:

\section{Contribution 1: Design and analysis of a community-oriented and context-aware opportunistic networking architecture for smart mobile platforms}

\section{Contribution 2: Design and analysis of a lightweight and scalable opportunistic ad hoc data dissemination protocol for smart mobile devices}

\section{Contribution 3: Evaluation of the presented protocols in different application areas}

Chapters 1-2, which constitute the background study of this thesis, provide an insight into the dynamics of the problem of opportunistic communications so as to analyze the challenges faced in the development of OppNet services. To this respect, the background study is helpful to understand why the contributions of this thesis are substantial as well as to clarify how the set of design criteria presented in Chapter 3 is developed. Regarding the development of OppNets, the currently-available wireless ad hoc networking standards on mobile devices, $\mathrm{Wi}-\mathrm{Fi}$ and Bluetooth, are surveyed in relation to their connectivity capabilities and support on current mobile O/S platforms. Furthermore, related works are scrutinized by explaining their focus of study in the protocol stack. 
It has been shown that current wireless connectivity schemes face with several challenges that are classified as $i$ ) design challenges and ii) operational challenges:

- The design challenges are mostly due to the restrictions and limitations reflected by mobile O/S platforms and their affiliated Wi-Fi and Bluetooth radio adapters. One one hand, the wireless interfaces are restricted, i.e. they are provisioned either as hidden features or with limited functionality. On the other hand, they are not strong enough to provide networking service if the number of devices are high.

- The operational challenges create another set of difficulties to perform networking with high scalability and adaptability in view of highly-dynamic characteristics of OppNets. It is shown that there has been a large number of forwarding approaches which try to eliminate the operational challenges in the transport layer by providing a level of efficient resource utilization.

The conclusion drawn from the background study is that utilizing connection-based methods with Wi-Fi and Bluetooth interfaces is insufficient to provide an efficient OppNet service. To provide an adaptive and serviceable connectivity between OppNet devices, all that is left is to utilize wireless network identifiers (simply beacons) as messages shared in the OppNet. Luckily, beacons provide several advantages in the wireless medium shared. Nevertheless, it is shown that the existing approaches utilizing beacons are very limited, and do not take the aforementioned challenges into account.

Chapter 3 is dedicated to introduce the Cocoon architecture, in which the networking schemes and corresponding functionalities corresponding to Contribution 1 are disclosed. The architecture adheres to proven principles, minimizes costs and maintenance requirements, and promotes extendibility and usability.

Chapter 4 presents Opportunistic Beacon Networking (OBN), which corresponds to Contribution 2. OBN is a lightweight solution for data forwarding and data dissemination in a multihop way. OBN exploits the widely-known concept of wireless broadcast advantage to the full extent. That is, data shared as opportunistic beacons can be received by multiple observers. To this end, OBN is a highly-opportunistic and highly-scalable method. With its ease of applicability, the protocol can directly operate on any smart mobile platform without requiring modifications on their affiliated wireless adapters. Therefore, it can be readily integrated to the mobile opportunistic sensing and networking applications. Moreover, inexperienced end users can easily take part in such applications.

To enable two-way and continuous communication between devices, a data switching protocol is devised. The protocol assigns switch operations to devices within specific intervals to make them alternate between the roles of beaconing and scanning. The protocol is comprehensively tested with different time parameters regarding the wireless operations used in the protocol. These time parameters are further used in the implementation of the model as a simulation model which is validated with real-world experiments.

An OBN deployment can involve single (1) or multiple (N) sources and destinations based on the application requirements. In a multipoint-to-multipoint (N-to-N) OBN type, all participating devices can share data with each other as presented in Chapter 4. Our results clearly 
indicate that OBN achieves high scalability in N-to-N scenarios under high device density. In addition, OBN may suit for the multipoint-to-point (N-to-1) scenarios having a central hub that continuously operates as a beacon observer to collect messages from available opportunistic beacons. Furthermore, our results also show that OBN provides high suitability for low message frequency scenarios, and for the networks having single (or limited number of) message sources. This means that OBN can be utilized in point-to-multipoint (1-to-N) and point-to-point (1-to-1) scenarios as well.

Supporting all above-mentioned communication types, OBN can be readily integrated to any group and type of smart mobile devices to render service to mobile sensing application types such as mobile lost \& found, participatory monitoring, proximity marketing, and so on.

Chapter 5, which corresponds to Contribution 3, offers a set of applications developed on top of the OBN model:

- An opportunistic multi-hop short message service intended for challenged environments. Named Oppline ${ }^{1}$, the service utilizes the OBN model to share simplistic messages between device pairs through other devices.

- A data dissemination application intended for vehicular environments. Named VADISS ${ }^{2}$, the application utilizes OBN to share traffic safety information between drivers, pedestrians, etc.

- A data dissemination application based on the dual radio utilization scheme offered for OBN. Named BLESSED ${ }^{3}$, the method is evaluated in several OppNets ranging from very sparse to very dense.

\subsubsection{Research Questions Answered}

With regard to the research questions introduced in Chapter 1, the key principles addressed in this thesis are as follows:

- Rapid information delivery: The concept of opportunistic beacons is introduced to enable and facilitate delay-tolerant networking between devices. Opportunistic beacons can operate on top of either Wi-Fi or Bluetooth protocol stack without requiring any manual configuration or adaptation in the utilized interface. Eliminating the connection establishment phase, opportunistic beacons enable lightweight and instantaneous data forwarding in ad hoc fashion and further regulates routing roles between devices.

- Self-configuring, self-organizing, self-healing network model: Based on opportunistic beacons, two communications models are supported in the architecture.

1. stands for opportunistic line, a connectivity state between online and offline.

2. stands for Vehicular Ad Hoc Data Dissemination, or SSID Advertisements for Vehicular Networking when read backwards.

3. stands for BLE profile and IEEE 802.11 Service Set Encoding-based Dissemination 
- Connection-free networking, namely OBN which is given in Chapter 4. The performance evaluation of OBN has been conducted through real-world experiments and validated simulations. From small-scale to large-scale, from static to mobile, from dense to sparse, OBN is investigated under different network setups and with several evaluation parameters. Our results clearly indicate that OBN achieves high scalability for any dissemination scenario under reasonable device density. The OBN protocol is limited in terms of throughput. To mitigate this limitation, the following enhancements are proposed and tested:

* Smart encodings for opportunistic beacons, to increase the amount of information fitted in the wireless network identifiers.

* A message-adaptive forwarding scheme, which takes the number of received opportunistic beacons into account to adapt switching times.

* A time-adaptive forwarding scheme, which distributively schedules switching times based on time advertisements.

* Dual radio utilization, to complement opportunistic beacons with scanning and beacon observers with advertising. The wireless PHY/MAC protocols used are Wi-Fi and BLE.

For each of the enhancement proposed, a level of improvement is achieved in the networking performance.

- Connection-based networking, which exploits opportunistic beacons to assist the progress of connection establishments between devices. This model is explained within the Cocoon architecture.

- Energy efficiency: It is shown that with the networking models presented, a significant power efficiency is achieved compared to conventional connectivity schemes such as Wi-Fi Ad Hoc mode and Bluetooth.

- Multiple application support: Cocoon enables a fully-compatible operation on any type of modern mobile device ranging from, but not limited to, smartphones to smartwatches to tablets. Cocoon expedites several opportunistic data sharing and dissemination tasks between devices in ad hoc fashion, and is able to schedule these tasks with an adaptive service management.

- QoS model: With the exploitation of current network context and in deference to affiliated application requirements, Cocoon employs an analytic decision-making model on the concurrently running tasks. This model regulates the order of tasks and selects a networking strategy appropriate for the present network characteristics. For the model development, a new set of QoS requirements are defined.

- Development and analysis tools: The Cocoon architecture is implemented as an Android service. Furthermore, its networking models are implemented in the Matlab as a simulation called Oppliqué.

- Cocoon API: The details of the service is given in Appendix C.

- Oppliqué: The details of the simulation is given in Appendix B. 


\subsubsection{LesSONS LEARNED}

The following briefly outlines the lessons learned during this endeavor:

- Sometime somewhere, delay is preferable to error. For challenged networking purposes, taking time in delivery might be better than having a failure in express delivery. Especially in critical areas, therefore, OppNets can be highly helpful where conventional networking approaches might not operate well.

- The medium is the message. And, thanks to the ever-growing wireless beacon technology, the message is the medium. Beacons can be exploited as simple sensing units for networking, e.g. they can inform the presence, status, and various properties of their affiliated medium without requiring burdensome connectivities. As a result, a multitude of beacons, each carrying information about its medium, can expand to wider media.

- The dedicated wireless ad hoc protocols (e.g. Wi-Fi Ad Hoc mode), despite being improved by their working groups, will apparently remain as hidden features in smart mobile O/S platforms. The biggest reason is the possibility of malicious uses that may threaten the security of communications in a locality.

- It appears to be that products from different vendors (e.g. Apple, Google) will have divergent specifications for their own hardware and software. Therefore, a generic OppNet design that clears the hurdle of possible platform conflicts is significant.

- In order to increase the popularity of OppNets, appealing incentives are necessary. To widen people's interest, access, and participation in OppNet applications, a simple and efficient implementation is the biggest incentive. 


\subsection{Open Research Directions}

This thesis has presented the first development of a fully-universal opportunistic communication architecture in the research field of OppNets. Furthermore, this thesis has provided the first investigation of beacon utilization for the use of opportunistic communications. Apart from versatile functionalities, several additions and several improvements are proposed in the presented networking architecture. Nevertheless, there is technologically a wide room for further improvements. The open research issues are briefly discussed in the following:

\subsubsection{Broadening the Context}

The context utilized in Cocoon is only related to routing- and QoS-specific information. Nevertheless, application-specific or user-generated information might be directly used in the QoS model to increase context-awareness. In addition, an opportunistic sensing scheme might improve the networking service operation. With an increased context awareness, the presented forwarding mechanisms can be improved as well, for example they can be converted to directional or geographical forwarding strategies.

\subsubsection{INFRASTRUCTURE}

This thesis has focused solely on the case of infrastructure-less networks, regarding the presented protocols and their applications in ad hoc fashion. Our performance evaluation results have clearly shown that both data dissemination and routing efficiency dramatically drops as the network sparsity increases. However, utilizing a fixed or a mobile backbone in an OppNet will positively affect connectedness between participating devices. One straightforward example is to assign fixed stations such as APs and RSUs the task of storing and rebroadcasting data later on to other devices passing by. This would yield certain benefits in terms of delivery ratio and delay. In our view, due to the large amount of data that devices (or users) can generate, OppNets can handover some amount of their traffic to situated networks, thereby making a hybrid structure available for the presented protocols and related applications in this thesis.

\subsubsection{Complex Scenarios}

For several complex scenarios, the Cocoon architecture can be enhanced in a way that it accomplishes multiple objectives of different OppNet applications at the same time. This can be an additional feature of the multiple application support presented in the architecture. For example, a group of OppNet applications functioning in a same environment can be converged on a common operation regardless of their individual objectives. This can be achieved by a smart functionality added to opportunistic beacons. To say more clearly, opportunistic beacons can define a shared objective in an OppNet, can further assign differentiated jobs for each device with smart advertisements. This necessitates a broader context utilization. 


\subsubsection{SECURITY}

Security considerations are out of thesis scope and therefore have not been addressed in the architecture. One of the main features of OppNets is the lack of an infrastructure, which could be considered as a critical aspect from a security standpoint. On the other hand, dynamic nature of OppNets makes it difficult to apply security protocols presented for traditional networks. As a matter of fact, mobile devices form OppNets without the help of any security infrastructure and without any prior trust between themselves. Ensuring security in such cases is not a trivial task. Security services can be provided in the architecture by allowing communications only in closed/private communities, so that users can trust each other. However, such a solution goes against the openness of OppNet application and services to public use.

\subsubsection{The Future of Beacons}

The future of the beacon technology is full of opportunities. Particularly being developed for the use of proximity-based services, beacons are being multiplied around us each passing day. The support for lightweight and versatile beaconing functionality in wireless networking standards is increasing as well. As mentioned previously, BLE is the first standard that provides this support in mobile devices. The availability of BLE is steeply increasing in connected devices. The Bluetooth Special Interest Group estimate that more than 90 percent of the smartphones will have BLE support by 2018 [39]. Like BLE, which is the latest Bluetooth standard dedicated for proximity-based services, Wi-Fi technology has been evolving alongside energy-efficient proximity-based social networking services.

In the very near future, Wi-Fi Aware will be released as a standard in commercially offthe-shelf mobile devices [141]. The ability of Wi-Fi Aware to send and receive tiny beacons before establishing a connection will further enable a two-way opportunistic lightweight communications among devices. This capability will not only enable a user to discover nearby information and services, but also provide sharing of various information types in an efficient and effective manner. On the other hand, Wi-Fi Halow has been introduced another type of low-power and increased long-range beacon technology to enable an assortment of new efficient use cases in the smart home, connected car, and digital healthcare, as well as industrial, retail, and smart city environments [142].

The advancing beacon technology can be utilized to eliminate the presented limitations in the Cocoon architecture. It is worth to mention in this point that beacon technology is particularly designed for single-hop arrangements. Cocoon can easily extend the scalability of beacons in a power-efficient, versatile, but most importantly multi-hop way. First, with smart beacons, the networking protocol will achieve considerably shorter delays. Second, the one-directional neighbor discovery will be obsolete. Third, integration to fixed infrastructures will be easier. Overall, the beacons will provide higher operability as they advance towards a higher ubiquity and functionality. 


\subsection{Final Remarks}

Opportunities in opportunistic communications are increasing generously. As OppNets edge closer to reality, the supporting applications and services must also evolve in accordance with public demand. The worldwide success of smart mobile technologies can, in large part, be contributed to the ever-evolving requirements of our mobile lifestyle. To this respect, there is still need for smarter kind of OppNet applications to make people stay connected to any kind of information in their social space. Therefore, it is a responsibility for everyone to think outside of the box to make us stay safely and comfortably in the cocoon of modern society. 



\section{Bibliography}

[1] L. Pelusi, A. Passarella, and M. Conti. Opportunistic networking: data forwarding in disconnected mobile ad hoc networks Communications Magazine, 44(11):134-141, November 2006. ISSN 0163-6804. doi: 10.1109/MCOM.2006.248176.

[2] B. Guo, D. Zhang, Z. Wang, Z. Yu, and X. Zhou. Opportunistic iot: Exploring the harmonious interaction between human and the internet of things. Journal of Network and Computer Applications, 36(6):1531 - 1539, November 2013. ISSN 1084-8045. doi: 10.1016/j.jnca.2012.12.028.

[3] M. Conti and S. Giordano. Mobile ad hoc networking: milestones, challenges, and new research directions. IEEE Communications Magazine, 52(1):85-96, January 2014. ISSN 0163-6804. doi: 10.1109/MCOM.2014.6710069.

[4] C. Watson, J. McCarthy, and J. Rowley. Consumer attitudes towards mobile marketing in the smart phone era. International Journal of Information Management, 33(5):840 - 849, 2013. ISSN 0268-4012. doi: 10.1016/j.ijinfomgt.2013.06.004

[5] Forbes Article: Apple iOS And Google Android Smartphone Market Share Flattening. http:/ / www.forbes.com/sites/dougolenick/2015/05/27/apple-ios-and-google-android-smartphone-market-shareflattening-idc. Last accessed on May 6, 2016.

[6] Ericsson Mobility Report - November 2015. http://www.ericsson.com/res/docs/2015/mobility-report/ericsson-mobilityreport-nov-2015.pdf. Last accessed on May 6, 2016

[7] Cisco Visual Networking Index 2015. http://www.cisco.com/c/en/us/solutions/collateral/service-provider/visualnetworking-index-vni/mobile-white-paper-c11-520862.pdf. Last accessed on May 6, 2016.

[8] J. Kim, J. Lee, J. Kim, and J. Yun. M2m service platforms: Survey, issues, and enabling technologies. IEEE Communications Surveys Tutorials, 16(1):61-76, January 2014. ISSN 1553-877X. doi: 10.1109/SURV.2013.100713.00203.

[9] G. Zyba, G. M. Voelker, S. Ioannidis, and C. Diot. Dissemination in opportunistic mobile ad-hoc networks: The power of the crowd. In INFOCOM, 2011 Proceedings IEEE, pages 1179-1187, April 2011. doi: 10.1109/INFCOM.2011.5934896.

[10] H. Nishiyama, M. Ito, and N. Kato. Relay-by-smartphone: realizing multihop device-to-device communications. IEEE Communications Magazine, 52(4):56-65, April 2014. ISSN 0163-6804. doi: 10.1109/MCOM.2014.6807947.

[11] Z. J. Haas, J. Deng, B. Liang, P. Papadimitratos, and S. Sajama. Wireless ad hoc Networks. John Wiley Sons, Inc., April 2003. ISBN 9780471219286. doi: 10.1002/0471219282.eot185.

[12] C. E. Perkins. Ad Hoc Networking. Addison-Wesley Professional, 1 edition, August 2008. ISBN 0321579070, 9780321579072.

[13] I. Chlamtac, M. Conti, and J. J.-N. Liu. Mobile ad hoc networking: imperatives and challenges. Ad Hoc Networks, 1(1):13 - 64, July 2003. ISSN 1570-8705. doi: http:/ /dx.doi.org/10.1016/S1570-8705(03)00013-1.

[14] C. Yu, B. Lee, and H. Yong Youn. Energy efficient routing protocols for mobile ad hoc networks. Wireless Communications and Mobile Computing, 3(8):959-973, 2003. ISSN 1530-8677. doi: 10.1002/wcm.119.

[15] K. Fall. A delay-tolerant network architecture for challenged internets. In Proceedings of the 2003 Conference on Applications, Technologies, Architectures, and Protocols for Computer Communications, SIGCOMM '03, pages 27-34, New York, NY, USA, 2003. ACM. ISBN 1-58113-735-4. doi: 10.1145/863955.863960.

[16] Z. Zhang. Routing in intermittently connected mobile ad hoc networks and delay tolerant networks: overview and challenges. IEEE Communications Surveys Tutorials, 8(1):24-37, January 2006. ISSN 1553-877X. doi: 10.1109/COMST.2006.323440.

[17] Y. K. Ip, W. C. Lau, and O. C. Yue. Forwarding and replication strategies for dtn with resource constraints. In Vehicular Technology Conference, 2007. VTC2007-Spring. IEEE 65th, pages 1260-1264, April 2007. doi: 10.1109/VETECS.2007.265. 
[18] K. A. Harras, K. C. Almeroth, and E. M. Belding-Royer. NETWORKING 2005. Networking Technologies, Services, and Protocols; Performance of Computer and Communication Networks; Mobile and Wireless Communications Systems: 4th International IFIP-TC6 Networking Conference, Waterloo, Canada, May 2-6, 2005. Proceedings, chapter Delay Tolerant Mobile Networks (DTMNs): Controlled Flooding in Sparse Mobile Networks, pages 1180-1192. Springer Berlin Heidelberg, 2005. ISBN 978-3-540-32017-3. doi: 10.1007/11422778_95.

[19] Z. J. Haas and T. Small. Evaluating the capacity of resource-constrained dtns. In Proceedings of the 2006 International Conference on Wireless Communications and Mobile Computing, IWCMC '06, pages 545-550, New York, NY, USA, 2006. ACM. ISBN 1-59593306-9. doi: 10.1145/1143549.1143658.

[20] T. Hossmann, F. Legendre, and T. Spyropoulos. From contacts to graphs: Pitfalls in using complex network analysis for dtn routing. In INFOCOM Workshops 2009, IEEE, pages 1-6, April 2009. doi: 10.1109/INFCOMW.2009.5072147.

[21] V. Manfredi, M. Crovella, and J. Kurose. Understanding stateful vs stateless communication strategies for ad hoc networks. In Proceedings of the 17th Annual International Conference on Mobile Computing and Networking, MobiCom '11, pages 313-324, New York, NY, USA, 2011. ACM. ISBN 978-1-4503-0492-4. doi: 10.1145/2030613.2030649.

[22] M. Weiser. The computer for the 21st century. Scientific American, 265(3):94-104, 1991. doi: 10.1038/scientificamerican0991-94.

[23] I. F. Akyildiz and I. H. Kasimoglu. Wireless sensor and actor networks: research challenges. Ad Hoc Networks, 2(4):351 - 367, 2004. ISSN 1570-8705. doi: http://dx.doi.org/10.1016/j.adhoc.2004.04.003.

[24] A. Pietilänen and C. Diot. Dissemination in opportunistic social networks: The role of temporal communities. In Proceed ings of the Thirteenth ACM International Symposium on Mobile Ad Hoc Networking and Computing, MobiHoc '12, pages 165-174, New York, NY, USA, 2012. ACM. ISBN 978-1-4503-1281-3. doi: 10.1145/2248371.2248396.

[25] M. R. Schurgot, C. Comaniciu, and K. Jaffrès-Runser. Beyond traditional DTN routing: Social networks for opportunistic communication. CoRR, abs/1110.2480, 2011. doi: 10.1109/MCOM.2012.6231292.

[26] B. Han, P. Hui, V. S. A. Kumar, M. V. Marathe, J. Shao, and A. Srinivasan. Mobile data offloading through opportunistic communications and social participation. IEEE Transactions on Mobile Computing, 11(5):821-834, May 2012. ISSN $1536-1233$. doi: 10.1109/TMC.2011.101.

[27] A. Aijaz, H. Aghvami, and M. Amani. A survey on mobile data offloading: technical and business perspectives. IEEE Wireless Communications, 20(2):104-112, April 2013. ISSN 1536-1284. doi: 10.1109/MWC.2013.6507401.

[28] P. Jänis, C. Yu, K. Doppler, C. Ribeiro, C. Wijting, K. Hugl, O. Tirkkonen, and V. Koivunen. Device-to-device communication underlaying cellular communications systems. International Journal of Communications, Network and System Sciences, 2(3):169, 2009. doi: 10.4236/ijens.2009.23019.

[29] S. Trifunovic, B. Distl, D. Schatzmann, and F. Legendre. Wifi-opp: Ad-hoc-less opportunistic networking. In ACM-CHANTS 2011, pages 37-42, 2011. ISBN 978-1-4503-0870-0. doi: 10.1145/2030652.2030664.

[30] G. Anastasi, E. Borgia, M. Conti, and E. Gregori. Wi-fi in ad hoc mode: a measurement study. In Pervasive Computing and Communications, 2004. PerCom 2004. Proceedings of the Second IEEE Annual Conference on, pages 145-154, March 2004. doi: 10.1109/PERCOM.2004.1276853.

[31] D.J. Dubois, Y. Bando, K. Watanabe, and H. Holtzman. Lightweight self-organizing reconfiguration of opportunistic infrastructure-mode wifi networks. In Self-Adaptive and Self-Organizing Systems (SASO), 2013 IEEE 7th International Conference on, pages 247-256, Sept 2013. doi: 10.1109/SASO.2013.41.

[32] D. Contreras and M. Castro. Experimental assessment of the adequacy of bluetooth for opportunistic networks. Ad Hoc Networks, 25, Part B:444 - 453, 2015. ISSN 1570-8705. doi: 10.1016/j.adhoc.2014.08.007.

[33] S. Sagari, A. Baid, I. Seskar, T. Murase, M. Oguchi, and D. Raychaudhuri. Performance evaluation of mobile hotspots in densely deployed wlan environments. In IEEE PIMRC 2013, pages 2935-2939, 2013. doi: 10.1109/PIMRC.2013.6666649.

[34] Stuart T. and Tine C. Understanding the changing mobile user: Gain insights from cisco's mobile consumer research, cisco systems. Cisco White Paper, 2013. URL http://www.cisco.com/c/en/us/solutions/collateral/ service-provider/service-provider-wi-fi/white-paper-c11-729796.pdf.

[35] J. Haartsen, M. Naghshineh, J. Inouye, O. J. Joeressen, and W. Allen. Bluetooth: Vision, goals, and architecture. SIGMOBILE Mob. Comput. Commun. Rev., 2(4):38-45, October 1998. ISSN 1559-1662. doi: 10.1145/1321400.1321402.

[36] Q. Xia, X. Jin, and M. Hamdi. Cross layer design for the ieee 802.11 wlans: Joint rate control and packet scheduling. IEEE Transactions on Wireless Communications, 6(7):2732-2740, July 2007. ISSN 1536-1276. doi: 10.1109/TWC.2007.06019.

[37] Wi-Fi P2P Technical Specification v1.5. http://www.wi-fi.org/downloads-registered/Wi-Fi_P2P_Technical_Specification_v1.5.pdf, . Last accessed on May 6, 2016. 
[38] C. Gomez, J. Oller, and J. Paradells. Overview and evaluation of bluetooth low energy: An emerging low-power wireless technology. Sensors, 12(9):11734-11753, 2012. doi: 10.3390/s120911734.

[39] Mobile Telephony Market - Bluetooth SIG. https://www.bluetooth.com/marketing-branding/markets/mobile-phonessmart-phones, . Last accessed on May 6, 2016.

[40] M. Orlinski and N. Filer. Neighbour discovery in opportunistic networks. Ad Hoc Networks, 25, Part B:383 - 392, 2015. ISSN 1570-8705. doi: http://dx.doi.org/10.1016/j.adhoc.2014.07.024. New Research Challenges in Mobile, Opportunistic and Delay-Tolerant NetworksEnergy-Aware Data Centers: Architecture, Infrastructure, and Communication.

[41] S. Trifunovic, M. Kurant, K. A. Hummel, and F. Legendre. Wlan-opp: Ad-hoc-less opportunistic networking on smartphones Ad Hoc Networks, 25, Part B:346 - 358, 2015. ISSN 1570-8705. doi: 10.1016/j.adhoc.2014.07.011.

[42] J. Jiang, B. Lin, and Y. Tseng. Analysis of bluetooth device discovery and some speedup mechanisms. Journal of the Institute of Electrical Engineering, 11(4):301-310, 2004.

[43] D. Camps-Mur, A. Garcia-Saavedra, and P. Serrano. Device-to-device communications with wi-fi direct: overview and experimentation. Wireless Communications, IEEE, 20(3):96-104, June 2013. ISSN 1536-1284. doi: 10.1109/MWC.2013.6549288.

[44] The Serval Project. http://www.servalproject.org. Last accessed on May 6, 2016.

[45] Haggle: An innovative Paradigm for Autonomic Opportunistic Communication . http://www.haggleproject.org Last accessed on May 6, 2016.

[46] ICT-SCAMPI: Service Platform for Social Aware Mobile and Pervasive Computing. http://www.ict-scampi.eu. Last accessed on May 6, 2016.

[47] Open Garden. http://opengarden.com. Last accessed on May 6, 2016.

[48] PodNet - Mobile Distribution of User-generated Content. http://www.podnet.ee.ethz.ch. Last accessed on May 6, 2016.

[49] 7DS System. http://www1.cs.columbia.edu/ arezu/7DS/index.html. Last accessed on May 6, 2016.

[50] Socialnets. http://www.social-nets.eu. Last accessed on May 6, 2016.

[51] P. Gardner-Stephen, J. Lakeman, R. Challans, C Wallis, A Stulman, and Y Haddad. Meshms: Ad hoc data transfer within mesh network. Int. Journal of Communications, Network and System Sciences, 5(8):496-504, 2012. doi: 10.4236/ijcns.2012.58060.

[52] P. Gardner-Stephen and S. Palaniswamy. Serval mesh software-wifi multi model management. In Proceedings of the 1st International Conference on Wireless Technologies for Humanitarian Relief, ACWR '11, pages 71-77, New York, NY, USA, 2011. ACM. ISBN 978-1-4503-1011-6. doi: 10.1145/2185216.2185245.

[53] A. Neumann, C. Aichele, M. Lindner, and S. Wunderlich. Better approach to mobile ad-hoc networking (batman). IETF draft, October, 2008.

[54] M. Abolhasan, B. Hagelstein, and JC-P. Wang. Real-world performance of current proactive multi-hop mesh protocols. In Communications, 2009. APCC 2009. 15th Asia-Pacific Conference on, pages 44-47. IEEE, 2009. doi: 10.1109/APCC.2009.5375690.

[55] P. Hui, A. Chaintreau, J. Scott, R. Gass, J. Crowcroft, and C. Diot. Pocket switched networks and human mobility in conference environments. In Proceedings of the 2005 ACM SIGCOMM Workshop on Delay-tolerant Networking, WDTN '05, pages 244-251, New York, NY, USA, 2005. ACM. ISBN 1-59593-026-4. doi: 10.1145/1080139.1080142.

[56] Haggle version 0.4. http:/ / www.haggleproject.org. Last accessed on May 6, 2016.

[57] E. Nordstrom, C. Rohner, and P. Gunningberg. Haggle: Opportunistic mobile content sharing using search. Computer Communications, 48:121 - 132, 2014. ISSN 0140-3664. doi: http://dx.doi.org/10.1016/j.comcom.2014.03.017.

[58] E. Nordström, P. Gunningberg, and C. Rohner. Haggle: A data-centric network architecture for mobile devices. In Proceedings of the 2009 MobiHoc S3 Workshop on MobiHoc S3, MobiHoc S3 '09, pages 37-40, New York, NY, USA, 2009. ACM. ISBN 978-160558-521-5. doi: 10.1145/1540358.1540370.

[59] M. Papandrea, S. Vanini, and S. Giordano. A lightweight localization architecture and application for opportunistic networks. In World of Wireless, Mobile and Multimedia Networks Workshops, 2009. WoWMoM 2009. IEEE International Symposium on a, pages 1-3, June 2009. doi: 10.1109/WOWMOM.2009.5282438.

[60] A. Pietiläinen, E. Oliver, J. LeBrun, G. Varghese, and C. Diot. Mobiclique: Middleware for mobile social networking. In Proceedings of the 2Nd ACM Workshop on Online Social Networks, WOSN '09, pages 49-54, New York, NY, USA, 2009. ACM. ISBN 978-1-60558-445-4. doi: 10.1145/1592665.1592678. 
[61] A. Martín-Campillo, J. Crowcroft, E. Yoneki, R. Martí, and C. Martínez-García. Using haggle to create an electronic triage tag. In Proceedings of the Second International Workshop on Mobile Opportunistic Networking, MobiOpp '10, pages 167-170, New York, NY, USA, 2010. ACM. ISBN 978-1-60558-925-1. doi: 10.1145/1755743.1755775.

[62] O. Mokryn, D. Karmi, A. Elkayam, and T. Teller. Help me: Opportunistic smart rescue application and system. In Ad Hoc Networking Workshop (Med-Hoc-Net), 2012 The 11th Annual Mediterranean, pages 98-105, June 2012. doi: 10.1109/MedHocNet. 2012.6257129 .

[63] V. Arnaboldi, M. Conti, and F. Delmastro. Cameo: A novel context-aware middleware for opportunistic mobile social networks. Pervasive and Mobile Computing, 11(0):148 - 167, 2014. ISSN 1574-1192. doi: http://dx.doi.org/10.1016/j.pmcj.2013.09.010.

[64] C. Boldrini, M. Conti, F. Delmastro, and A. Passarella. Context- and social-aware middleware for opportunistic networks. Journal of Network and Computer Applications, 33(5):525 - 541, 2010. ISSN 1084-8045. doi: http:/ /dx.doi.org/10.1016/j.jnca.2010. 03.017 .

[65] A. Moghadam, S. Srinivasan, and H. Schulzrinne. 7ds - a modular platform to develop mobile disruption-tolerant applications In Next Generation Mobile Applications, Services and Technologies, 2008. NGMAST'08. The Second International Conference on, Sept 2008. doi: 10.1109/NGMAST.2008.75.

[66] M. May, V. Lenders, G. Karlsson, and C. Wacha. Wireless opportunistic podcasting: Implementation and design tradeoffs. In Proceedings of the Second ACM Workshop on Challenged Networks, CHANTS '07, pages 75-82, New York, NY, USA, 2007. ACM. ISBN 978-1-59593-737-7. doi: 10.1145/1287791.1287806.

[67] H. Wirtz, G. Kunz, J. Laudenberg, R. Backhaus, and K. Wehrle. High-performance, energy-efficient mobile wireless networking in 802.11 infrastructure mode. In Mobile Ad Hoc and Sensor Systems (MASS), 2014 IEEE 11th International Conference on, pages 291-299, Oct 2014. doi: 10.1109/MASS.2014.21.

[68] A. Al-Akkad, L. Ramirez, A. Boden, D. Randall, and A. Zimmermann. Help beacons: Design and evaluation of an ad-hoc lightweight s.o.s. system for smartphones. In Proceedings of the SIGCHI Conference on Human Factors in Computing Systems, CHI '14, pages 1485-1494, New York, NY, USA, 2014. ACM. ISBN 978-1-4503-2473-1. doi: 10.1145/2556288.2557002.

[69] Y. Mao, J. Wang, J.P. Cohen, and B. Sheng. Pasa: Passive broadcast for smartphone ad-hoc networks. In ICCCN 2014, pages 1-8, Aug 2014. doi: 10.1109/ICCCN.2014.6911820.

[70] P. Persson and Y. Jung. Nokia sensor: From research to product. In Proceedings of the 2005 Conference on Designing for User eXperience, DUX '05, New York, NY, USA, 2005. AIGA: American Institute of Graphic Arts. ISBN 1-59593-250-X.

[71] Beacon Friend Finder. http://bff-app.com. Last accessed on May 6, 2016.

[72] Z. Yang, B. Zhang, J. Dai, A.C. Champion, D. Xuan, and D. Li. E-smalltalker: A distributed mobile system for social networking in physical proximity. In Distributed Computing Systems (ICDCS), 2010 IEEE 30th International Conference on, pages 468-477, June 2010. doi: 10.1109/ICDCS.2010.56.

[73] J. P. Cohen. Wireless message dissemination via selective relay over bluetooth (mdsrob). CoRR, abs/1307.7814, 2013. URL http://arxiv.org/abs/1307.7814.

[74] Y. Shafranovich. Bluetooth data exchange between android phones without pairing. CoRR, abs/1507.00650, 2015. URL http://arxiv.org/abs/1507.00650.

[75] J. Teng, B. Zhang, X. Li, X. Bai, and D. Xuan. E-shadow: Lubricating social interaction using mobile phones. Computers, IEEE Transactions on, 63(6):1422-1433, June 2014. ISSN 0018-9340. doi: 10.1109/TC.2012.290.

[76] FireChat. http://opengarden.com/firechat. Last accessed on May 6, 2016.

[77] Getting Started with iBeacon. https://developer.apple.com/ibeacon/Getting-Started-with-iBeacon.pdf. Last accessed on May 6, 2016.

[78] AltBeacon: The Open and Interoperable Proximity Beacon Specification. http://altbeacon.org. Last accessed on May 6, 2016.

[79] EddyStone Specification from Google. https://github.com/google/eddystone. Last accessed on May 6, 2016.

[80] Estimote Beacons. http://estimote.com. Last accessed on May 6, 2016.

[81] Gimbal Beacons and Firmware. http://gimbal.com. Last accessed on May 6, 2016.

[82] URIBeacon Software and Specification. http://uribeacon.io. Last accessed on May 6, 2016. 
[83] S. Cho and C. Julien. Chitchat: Navigating tradeoffs in device-to-device context sharing.

[84] T. Spyropoulos, K. Psounis, and C. S. Raghavendra. Efficient routing in intermittently connected mobile networks: The multiple-copy case. IEEE/ACM Transactions on Networking, 16(1):77-90, Feb 2008. ISSN 1063-6692. doi: 10.1109/TNET.2007. 897964.

[85] A. Vahdat, D. Becker, et al. Epidemic routing for partially connected ad hoc networks. Technical report, Technical Report CS-200006, Duke University, 2000.

[86] T. Spyropoulos, K. Psounis, and C. S. Raghavendra. Spray and wait: an efficient routing scheme for intermittently connected mobile networks. In Proceedings of the 2005 ACM SIGCOMM workshop on Delay-tolerant networking, WDTN '05, pages 252-259. ACM, 2005. ISBN 1-59593-026-4. doi: 10.1145/1080139.1080143.

[87] T. Spyropoulos, K. Psounis, and C. S. Raghavendra. Spray and focus: Efficient mobility-assisted routing for heterogeneous and correlated mobility. In Pervasive Computing and Communications Workshops, 2007. PerCom Workshops' 07. Fifth Annual IEEE International Conference on, pages 79-85. IEEE, March 2007. doi: 10.1109/PERCOMW.2007.108.

[88] J. Burgess, B. Gallagher, D. Jensen, and B. N. Levine. Maxprop: Routing for vehicle-based disruption-tolerant networks. In INFOCOM 2006. 25th IEEE International Conference on Computer Communications. Proceedings, pages 1-11, April 2006. doi: 10.1109/INFOCOM.2006.228.

[89] A. Lindgren, A. Doria, and O. Schelen. Probabilistic routing in intermittently connected networks. In Service Assurance with Partial and Intermittent Resources, pages 239-254. Springer, 2004. doi: 10.1145/961268.961272.

[90] A. Balasubramanian, B. Levine, and A. Venkataramani. Dtn routing as a resource allocation problem. In ACM SIGCOMM Computer Communication Review, volume 37, pages 373-384. ACM, 2007. doi: 10.1145/1282427.1282422.

[91] S. C. Nelson, M. Bakht, and R. Kravets. Encounter-based routing in dtns. In INFOCOM 2009, IEEE, pages 846-854. IEEE, April 2009. doi: 10.1109/INFCOM.2009.5061994.

[92] S. Jain, K. Fall, and R. Patra. Routing in a delay tolerant network, volume 34 of SIGCOMM '04. ACM, New York, NY, USA, 2004. ISBN 1-58113-862-8. doi: 10.1145/1015467.1015484.

[93] J. Leguay, T. Friedman, and V. Conan. Dtn routing in a mobility pattern space. In Proceedings of the 2005 ACM SIGCOMM workshop on Delay-tolerant networking, WDTN '05, pages 276-283. ACM, 2005. ISBN 1-59593-026-4. doi: 10.1145/1080139. 1080146

[94] W. Hsu, D. Dutta, and A. Helmy. Profile-cast: Behavior-aware mobile networking. In Wireless Communications and Networking Conference, 2008. WCNC 2008. IEEE, pages 3033-3038. IEEE, March 2008. doi: 10.1109/WCNC.2008.530.

[95] Q. Yuan, I. Cardei, and J. Wu. Predict and relay: An efficient routing in disruption-tolerant networks. In Proceedings of the Tenth ACM International Symposium on Mobile Ad Hoc Networking and Computing, MobiHoc '09, pages 95-104, New York, NY, USA, 2009. ACM. ISBN 978-1-60558-624-3. doi: 10.1145/1530748.1530762.

[96] S. Moon and A. Helmy. Understanding periodicity and regularity of nodal encounters in mobile networks: A spectral analysis In Global Telecommunications Conference (GLOBECOM 2010), 2010 IEEE, pages 1-5, Dec 2010. doi: 10.1109/GLOCOM.2010. 5684252 .

[97] Z. Wang, Mario A. Nascimento, and M. H. MacGregor. Discovering periodic patterns of nodal encounters in mobile networks Pervasive and Mobile Computing, 9(6):892 - 912, 2013. ISSN 1574-1192. doi: 10.1016/j.pmcj.2013.09.011. Mobile Data Challenge.

[98] W. Moreira, P. Mendes, and S. Sargento. Opportunistic routing based on daily routines. In World of Wireless, Mobile and Multimedia Networks (WoWMoM), 2012 IEEE International Symposium on a, pages 1-6, June 2012. doi: 10.1109/WoWMoM.2012 6263749

[99] W. Gao, G. Cao, T. La Porta, and J. Han. On exploiting transient social contact patterns for data forwarding in delay-tolerant networks. Mobile Computing, IEEE Transactions on, 12(1):151-165, Jan 2013. ISSN 1536-1233. doi: 10.1109/TMC.2011.249.

[100] X. Zhang and G. Cao. Transient community detection and its application to data forwarding in delay tolerant networks. In Network Protocols (ICNP), 2013 21st IEEE International Conference on, pages 1-10, Oct 2013. doi: 10.1109/ICNP.2013.6733594.

[101] P. Hui and J. Crowcroft. How small labels create big improvements. In Pervasive Computing and Communications Workshops, 2007. PerCom Workshops' 07. Fifth Annual IEEE International Conference on, pages 65-70. IEEE, March 2007. doi: 10.1109/PERCOMW. 2007.55 .

[102] E. M Daly and M. Haahr. Social network analysis for routing in disconnected delay-tolerant manets. In Proceedings of the 8th ACM international symposium on Mobile ad hoc networking and computing, pages 32-40. ACM, 2007. ISBN 978-1-59593-684-4. doi: $10.1145 / 1288107.1288113$ 
[103] A. Mtibaa, M. May, C. Diot, and M. Ammar. Peoplerank: social opportunistic forwarding. In INFOCOM, 2010 Proceedings IEEE, pages 1-5. IEEE, March 2010. doi: 10.1109/INFCOM.2010.5462261.

[104] P. Hui, J. Crowcroft, and E. Yoneki. Bubble rap: Social-based forwarding in delay-tolerant networks. Mobile Computing, IEEE Transactions on, 10(11):1576-1589, November 2011. ISSN 1536-1233. doi: 10.1109/TMC.2010.246.

[105] V. Etter, M. Kafsi, and E. Kazemi. Been there, done that: What your mobility traces reveal about your behavior. In Nokia Mobile Data Challenge 2012 Workshop. p. Dedicated task, volume 2, 2012

[106] K. A. Hummel and A. Hess. Estimating human movement activities for opportunistic networking: A study of movement features. In World of Wireless, Mobile and Multimedia Networks (WoWMoM), 2011 IEEE International Symposium on a, pages 1-7. IEEE, June 2011. doi: 10.1109/WoWMoM.2011.5986468.

[107] J. McInerney, S. Stein, A. Rogers, and N. R. Jennings. Breaking the habit: Measuring and predicting departures from routine in individual human mobility. Pervasive and Mobile Computing, 2013. ISSN 1574-1192. doi: http://dx.doi.org/10.1016/j.pmcj 2013.07.016

[108] J. Wang and B. Prabhala. Periodicity based next place prediction. In Nokia Mobile Data Challenge 2012 Workshop. p. Dedicated task, volume 2, 2012

[109] S. Gambs, M. Killijian, and Miguel N. del Prado C. Next place prediction using mobility markov chains. In Proceedings of the First Workshop on Measurement, Privacy, and Mobility, page 3. ACM, 2012. ISBN 978-1-4503-1163-2. doi: 10.1145/2181196.2181199.

[110] W. Mathew, R. Raposo, and B. Martins. Predicting future locations with hidden markov models. In Proceedings of the 2012 ACM Conference on Ubiquitous Computing, pages 911-918, New York, NY, USA, 2012. ACM. ISBN 978-1-4503-1224-0. doi: $10.1145 / 2370216.2370421$

[111] P. R. Pereira, A. Casaca, J. J. P. C. Rodrigues, V. N. G. J. Soares, Joan Triay, and Cristina Cervello-Pastor. From delay-tolerant networks to vehicular delay-tolerant networks. Communications Surveys Tutorials, IEEE, 14(4):1166-1182, Fourth 2012. ISSN 1553-877X. doi: 10.1109/SURV.2011.081611.00102.

[112] V. Conan, J. Leguay, and T. Friedman. Characterizing pairwise inter-contact patterns in delay tolerant networks. In Proceed ings of the 1st International Conference on Autonomic Computing and Communication Systems, Autonomics '07, pages 19:1-19:9, ICST, Brussels, Belgium, Belgium, 2007. ICST (Institute for Computer Sciences, Social-Informatics and Telecommunications Engineering). ISBN 978-963-9799-09-7.

[113] A. Passarella and M. Conti. NETWORKING 2011: 10th International IFIP TC 6 Networking Conference, Valencia, Spain, May 9-13, 2011, Proceedings, Part II, chapter Characterising Aggregate Inter-contact Times in Heterogeneous Opportunistic Networks, pages 301-313. Springer Berlin Heidelberg, Berlin, Heidelberg, 2011. ISBN 978-3-642-20798-3. doi: 10.1007/ 978-3-642-20798-3_23.

[114] T. Hossmann, T. Spyropoulos, and F. Legendre. Putting contacts into context: Mobility modeling beyond inter-contact times. In Proceedings of the Twelfth ACM International Symposium on Mobile Ad Hoc Networking and Computing, MobiHoc '11, pages 18:1-18:11, New York, NY, USA, 2011. ACM. ISBN 978-1-4503-0722-2. doi: 10.1145/2107502.2107526.

[115] C. Boldrini and A. Passarella. Hcmm: Modelling spatial and temporal properties of human mobility driven by users' social relationships. Computer Communications, 33(9):1056 - 1074, 2010. ISSN 0140-3664. doi: http://dx.doi.org/10.1016/j.comcom. 2010.01.013.

[116] T. Phe-Neau, M. Dias de Amorim, and V. Conan. The strength of vicinity annexation in opportunistic networking. In INFOCOM, 2013 Proceedings IEEE, pages 3369-3374, April 2013. doi: 10.1109/INFCOM.2013.6567166.

[117] S. Gaito, E. Pagani, and G. P. Rossi. Strangers help friends to communicate in opportunistic networks. Computer Networks, 55 (2):374 - 385, 2011. ISSN 1389-1286. doi: http://dx.doi.org/10.1016/j.comnet.2010.10.006. Wireless for the Future Internet.

[118] P. Yuan, H. Ma, and P. Duan. Impact of strangers on opportunistic routing performance. Journal of Computer Science and Technology, 28(3):574-582, 2013. ISSN 1860-4749. doi: 10.1007/s11390-013-1357-x.

[119] M. Musolesi and C. Mascolo. Car: context-aware adaptive routing for delay-tolerant mobile networks. Mobile Computing, IEEE Transactions on, 8(2):246-260, February 2009. ISSN 1536-1233. doi: 10.1109/TMC.2008.107.

[120] R. Chandra, J. Padhye, L. Ravindranath, and A. Wolman. Beacon-stuffing: Wi-fi without associations. In Mobile Computing Systems and Applications, 2007. HotMobile 2007. Eighth IEEE Workshop on, pages 53-57, March 2007. doi: 10.1109/HotMobile. 2007.16.

[121] M. Dürr, F. Gschwandtner, C. K. Schindhelm, and M. Duchon. Mobile Computing, Applications, and Services: Third International Conference, MobiCASE 2011, Los Angeles, CA, USA, October 24-27, 2011. Revised Selected Papers, chapter Secure and PrivacyPreserving Cross-Layer Advertising of Location-Based Social Network Services, pages 331-337. Springer Berlin Heidelberg, Berlin, Heidelberg, 2012. ISBN 978-3-642-32320-1. doi: 10.1007/978-3-642-32320-1_21. 
[122] S. Mathur and J. Zhang. Method for mobile terminal access to wireless lan based on access point services and service parameters, November 19 2013. US Patent 8,588,191.

[123] Ieee standard association - ieee get program. https://standards.ieee.org/getieee802/download/802.11-2012.pdf. Last accessed on May 6, 2016.

[124] Bluetooth core specification v4.2. https://www.bluetooth.org/docman/handlers/downloaddoc.ashx?doc_id=229737, Last accessed on May 6, 2016.

[125] Bluetooth accessory design guidelines for apple products. https://developer.apple.com/hardwaredrivers/BluetoothDesignGuidelines.pdf. Last accessed on May 6, 2016.

[126] B. Dezfouli, M. Radi, S. Abd Razak, K. Whitehouse, K. Abu Bakar, and T. Hwee-Pink. Improving broadcast reliability for neighbor discovery, link estimation and collection tree construction in wireless sensor networks. Computer Networks, 62:101 121, 2014. ISSN 1389-1286. doi: http://dx.doi.org/10.1016/j.comnet.2014.01.002.

[127] T. L. Saaty. The analytic network process. Springer, 2006.

[128] L. Yu and H. Liu. Efficient feature selection via analysis of relevance and redundancy. J. Mach. Learn. Res., 5, 2004.

[129] T. L. Saaty. Multi-criteria decision making. the analytic hierarchy process, pittsburgh, 1996

[130] M. Luna and R. Bott. Managing cache to prevent overloading of a wireless network due to user activity, October 172013. US Patent App. 13/912,067.

[131] N. Lu, N. Zhang, N. Cheng, X. Shen, J.W. Mark, and F. Bai. Vehicles meet infrastructure: Toward capacity x2013;cost tradeoffs for vehicular access networks. Intelligent Transportation Systems, IEEE Transactions on, 14(3):1266-1277, Sept 2013. ISSN 15249050. doi: 10.1109/TITS.2013.2258153.

[132] S. Al-Sultan, M. M. Al-Doori, A. H. Al-Bayatti, and H. Zedan. A comprehensive survey on vehicular ad hoc network. Journal of Network and Computer Applications, 37(0):380 - 392, 2014. ISSN 1084-8045. doi: http:/ /dx.doi.org/10.1016/j.jnca.2013.02.036.

[133] D. Jiang and L. Delgrossi. Ieee 802.11p: Towards an international standard for wireless access in vehicular environments. In Vehicular Technology Conference, Spring 2008. IEEE, pages 2036-2040, May 2008. doi: 10.1109/VETECS.2008.458.

[134] M. Amadeo, C. Campolo, and A. Molinaro. Enhancing ieee 802.11p/wave to provide infotainment applications in vanets. Ad Hoc Networks, 10(2):253 - 269, 2012. ISSN 1570-8705. doi: http://dx.doi.org/10.1016/j.adhoc.2010.09.013.

[135] J. Scott, P. Hui, J. Crowcroft, and C. Diot. Haggle: A networking architecture designed around mobile users. In Proceedings of the Third Annual IFIP Conference on Wireless On-Demand Network Systems and Services (WONS 2006). IEEE, January 2006.

[136] S. Hu, H. Liu, L. Su, H. Wang, T.F. Abdelzaher, P. Hui, W. Zheng, Z. Xie, and J.A. Stankovic. Towards automatic phone-tophone communication for vehicular networking applications. In INFOCOM, 2014 Proceedings IEEE, pages 1752-1760, April 2014. doi: 10.1109/INFOCOM.2014.6848113.

[137] F. Seraj, B. J. van der Zwaag, A. Dilo, T. Luarasi, and P. J. M. Havinga. Roads: A road pavement monitoring system for anomaly detection using smart phones. In Proceedings of the 1st International Workshop on Machine Learning for Urban Sensor Data, SenseML 2014, Nancy, France, Lecture Notes in Computer Science, pages 1-16, 2014.

[138] F. Seraj, K. Zhang, O. Turkes, N. Meratnia, and P. J. M. Havinga. A smartphone based method to enhance road pavement anomaly detection by analyzing the driver behavior. In Adjunct Proceedings of the 2015 ACM International Joint Conference on Pervasive and Ubiquitous Computing and Proceedings of the 2015 ACM International Symposium on Wearable Computers, UbiComp/ISWC'15 Adjunct, pages 1169-1177, New York, NY, USA, 2015. ACM. ISBN 978-1-4503-3575-1. doi: $10.1145 / 2800835.2800981$

[139] P.E. Uys, P.S. Els, and M. Thoresson. Suspension settings for optimal ride comfort of off-road vehicles travelling on roads with different roughness and speeds. Journal of Terramechanics, 44(2):163 - 175, 2007. ISSN 0022-4898. doi: 10.1016/j.jterra.2006.05. 002 .

[140] Fatjon Seraj, Kyle Zhang, Okan Turkes, Nirvana Meratnia, and Paul J. M. Havinga. A smartphone based method to enhance road pavement anomaly detection by analyzing the driver behavior. In Adjunct Proceedings of the 2015 ACM International Joint Conference on Pervasive and Ubiquitous Computing and Proceedings of the 2015 ACM International Symposium on Wearable Computers, UbiComp/ISWC'15 Adjunct, pages 1169-1177, New York, NY, USA, 2015. ACM. ISBN 978-1-4503-3575-1. doi: $10.1145 / 2800835.2800981$.

[141] Wi-Fi Aware by Wi-Fi Alliance. http://www.wi-fi.org/discover-wi-fi/wi-fi-aware, . Last accessed on May 6, 2016.

[142] Wi-Fi Halow by Wi-Fi Alliance. http://www.wi-fi.org/discover-wi-fi/wi-fi-halow, . Last accessed on May 6, 2016. 

APPENDIX A

Acronyms \& Notations 


\begin{tabular}{|c|c|}
\hline AI & Application Interdependence \\
\hline ANP & Analytic Network Process \\
\hline AM & Adapter Manager \\
\hline AP & Access Point \\
\hline API & Application Programming Interface \\
\hline ASCII & American Standard Code for Information Interchange \\
\hline Cocoon & Community-Oriented Context-Aware Opportunistic Networking \\
\hline $\mathrm{BR} / \mathrm{EDR}$ & Basic Rate/Enhanced Data Rate \\
\hline $\mathrm{BO}$ & Beacon Observer \\
\hline BLE & Bluetooth Low Energy, or Bluetooth Smart, or Bluetooth 4.0+ \\
\hline BLESSED & BLE Profile and 802.11 Service Set Encoding-based Dissemination \\
\hline BPU & Battery Percentage Usage \\
\hline BSS & Basic Service Set \\
\hline CA & Collision Avoidance \\
\hline CD & Contact Diversity \\
\hline CDMA & Code Division Multiple Access \\
\hline CI & Criteria Interdependence \\
\hline $\mathrm{CM}$ & Context Manager \\
\hline COTS & Commercially-Off-the-Shelf \\
\hline CS & Contact Stability \\
\hline CSMA & Carrier Sense Multiple Access \\
\hline DD & Data Diversity \\
\hline DS & Data Stability \\
\hline DTN & Delay-Tolerant Network \\
\hline FDMA & Frequency Division multiple Access \\
\hline GATT & Generic Attribute Protocol \\
\hline GPS & Global Positioning System \\
\hline GSM & Global System for Mobile Communications \\
\hline HTG & Hops-to-Go \\
\hline IBSS & Independent Basic Service Set \\
\hline IEEE & The Institute of Electrical and Electronics Engineers \\
\hline M2M & Machine-to-Machine \\
\hline MANET & Mobile Ad Hoc Network \\
\hline NF & Network Feedback \\
\hline NM & Network Manager \\
\hline $\mathrm{O} / \mathrm{S}$ & Operating System \\
\hline OAN & Opportunistic Association Networking \\
\hline OB & Opportunistic Beacon \\
\hline OBN & Opportunistic Beacon Networking \\
\hline Oppline & Opportunistic Line \\
\hline OppNet & Opportunistic Network \\
\hline $\mathrm{P} 2 \mathrm{P}$ & Peer-to-Peer \\
\hline PC & Personal Computer \\
\hline PHY /MAC & Physical \& Medium Access Control \\
\hline PF & Priority Feedback \\
\hline PRR & Packet Reception Rate \\
\hline
\end{tabular}




\begin{tabular}{|c|c|}
\hline PSN & Pocket-Switched Networks \\
\hline QoS & Quality-of-Service \\
\hline QoSM & QoS Manager \\
\hline RTT & Round Trip Time \\
\hline SMS & Short Message Service \\
\hline SSID & Service Set Identifier \\
\hline TDMA & Time Division Multiple Access \\
\hline TTL & Time-to-Live \\
\hline U\&S & Update \& Switch \\
\hline UTF & Unicode Transformation Format \\
\hline UUID & Universally-Unique Identifier \\
\hline VADISS & Vehicular Ad Hoc Data Dissemination via SSID \\
\hline Wi-Fi & Wireless Fidelity \\
\hline WLAN & Wireless Local Area Network \\
\hline WPAN & Wireless Personal Area Network \\
\hline WSN & Wireless Sensor Network \\
\hline$\alpha_{i}$ & i-th application \\
\hline$d_{i}$ & a-th node (device) \\
\hline$\mu B P U$ & Average BPU \\
\hline$A_{C}$ & Relative pairwise comparison matrix of a cluster $\mathrm{C}$ \\
\hline$D$ & Destination node \\
\hline$H_{n}(X)$ & Shannon's normalized entropy index for a given set $X$ \\
\hline$I_{A}$ & Application-specific data \\
\hline$I_{Q o S}$ & QoS-specific data \\
\hline$I_{R}$ & Routing-specific data \\
\hline$I(X, Y)$ & Mutual information index between two sets, $X$ and $Y$ \\
\hline$L\left(\alpha_{i}\right)$ & Capability level of i-th application \\
\hline$L\left(d_{a}\right)$ & Capability level of a-th node \\
\hline$p_{i}$ & Relay probability of i-th node \\
\hline$r_{i}$ & i-th relay node \\
\hline$S$ & Source node \\
\hline$t_{i}$ & Relay latency of i-th node \\
\hline$t_{B I}$ & Beacon Interval \\
\hline$t_{B O}$ & BO service duration \\
\hline$t_{M I}$ & Message (event) interval \\
\hline$t_{O B}$ & OB service duration \\
\hline$t_{S I}$ & Scan interval \\
\hline$t_{U \& S}$ & Update \& Switch duration \\
\hline$t_{X B O}$ & Transition duration from $\mathrm{OB}$ state to $\mathrm{BO}$ state \\
\hline$t_{X O B}$ & Transition duration from $\mathrm{BO}$ state to $\mathrm{OB}$ state \\
\hline$T$ & Period of an OB-BO cycle \\
\hline$w\left(t_{1}, t_{2}\right)$ & Stability index between two instances of same set at $t_{1}$ and $t_{2}$ \\
\hline & ANP supermatrix \\
\hline$W^{*}$ & ANP limit supermatrix \\
\hline
\end{tabular}





\section{APPENDIX B}

Oppliqué

API Guide 


\section{B.1 Overview}

This guide, the Opplique API Guide, introduces the classes, functions, and variables of the Oppliqué simulator. Furthermore, it briefly describes how to configure, run, and monitor setups in the simulator.

This guide is intended for anyone who wants to use Oppliqué API to programmatically develop Cocoon-based simulations in a MATLAB/Octave environment. The information in this guide is written for experienced developers who are familiar with network programming, especially with opportunistic networking. This guide also assumes familiarity with the Cocoon architecture. The definitions and examples given in this appendix are based on the implementation at the time of this writing, therefore online documents in the future may supersede this guide.

The most up-to-date technical documentation can be reached on the author's Github page: https://github.com/okanturkes/opplique

The Oppliqué simulator is a simulation environment that is capable of routing messages between nodes with various OppNet setups and sender and receiver types. It is developed to assist the development and analysis of simulated OppNet setups comprising smart mobile devices. It supports the classic OppNet schemes as well as involves the Cocoon-based networking approaches, i.e. Opportunistic Beacon Networking (OBN) and Opportunistic Association Networking (OAN) presented in this thesis.

At its core, the Opplique simulator is an agent-based discrete event simulation engine developed for MATLAB/Octave. At each simulation step, the engine updates a number of functions which implement the main simulation structures. The main structures of the simulator are the modeling of devices, device movements, message handling, inter-device contacts, networking, and opportunistic routing.

The operation of simulated devices are based on real-world measurements of the wireless operations. The simulator derives these operations with respect to a couple of device models for now such as Google Nexus 7, Samsung S2, Samsung S4, Samsung S4 Mini, and Motorola Moto G. As the collection of real-world measurements with other devices increases, the simulator will support more device models.

The simulator includes a couple of mobility models such as Random Waypoint Mobility Model, Random Walk Model, and Manhattan Grid to generate analyze mobility scenarios. These models are used as a tool for the investigation of mobile ad hoc network characteristics.

With its flexible input and output interfaces, the Oppliqué simulator can incorporate realworld traces and feeds from other mobility generators as well as generate mobility traces for use by other simulators.

The rest of this appendix is as follows: B.2 describes the packages. B.3 presents the main structures. B.4 describes the operation of the simulator and further explains how a setting file must be defined. B.5 gives two examples for simulation setup. 


\section{B.2 Packages}

In this section, the details of each package are given. The simulator has 5 main packages:

- Abstract

- Device

* Buffer

* Network

* Radio

* Routing

- Message

* Beacon

* Bundle
- Core

- FileHandler

- Preferences

- Settings

- UI

- Utils

- DATA

- Trace

- Wireless
- Models

- Device

- Mobility

- Simulation

- Environment

- Event

- Network

\section{B.2.1 ABSTRACT}

In this package, two structures, Device and Message, are defined.

\section{Device}

Each device in a simulation setup is defined as an instance of the Device structure and consists of a Buffer class for messages, a Radio class for the simulated wireless peripherals, a Network class for networking-related functions and variables, and a Routing class for routing-related ones.

Buffer class simulates a device buffer in order to store the received or created messages. As further explained, there are two types of messages, Beacon and Bundle; thereby there are two buffers defined in each device: BeaconList and BundleList.

- BeaconList stores the messages shared via wireless network identifiers. This buffer is directly used in OBN, and can be optionally used in OAN.

- BundleList stores the messages sent over connections, i.e. it stores the network packets. This buffer is only utilized in connection-based networks such as OAN.

Based on the general settings, each buffer can be defined with a specific size. Furthermore, multiple buffers of same type can be set for a device.

The following functions are defined under this class:

- Checklist (dID) is used to check expired messages in a list of the device dID.

- Insert (mID, dID) is used to add a message (mID) to a list of the device dID.

- Drop (mID, dID) is used to drop a message (mID) from a list of the device dID.

- Updatelist (dID) is used to update a list of the device dID. In the default version, this function shifts the order of messages of a list in a circular fashion. That is, the message at the end of the queue becomes the front-most message. This functionality is designed for the OBN buffer management implementation presented in Section 4.2. 
Radio class simulates the Wi-Fi and BLE interfaces of a Device structure. Based on the predefined settings of a simulation setup, each device can have Wi-Fi and/or BLE radios active for networking. In this class, the following functionalities are implemented as sub-classes of the interface object utilized:

- Association, which includes the functions and variables to simulate deviceto-device associations.

- Authentication, which includes the functions and variables to simulate device-todevice authentications.

- Connection, which includes the functions and variables to simulate device connections and Bundle transmissions.

- Disconnection, which includes the functions and variables to simulate device disconnections and to interrupt Bundle transmissions.

Network class is responsible to simulate neighbor discovery, OB-BO switches, and updating network information of a device over time.

- NetworkDiscovery includes the functions related to the beaconing and scanning of a Radio object.

- NetworkSwitcher includes the timing calculations for OB-BO switches. For traditional ad hoc connectivity setups, this class is not used.

- NetworkUpdater includes the functions which keep track of the network status of a device.

Routing class consists of functions related to forwarding. Several forwarding methods such as Epidemic [85], Direct Delivery, First Contact, PRoPHET [89], Spray \& Wait [86], and RoRo$L T^{*}$ are implemented under this class. These forwarding protocols can be applied for either Beacon or Bundle type of messaging in a network. Interested developers can implement their own forwarding approaches under this class.

\section{Message}

A Message structure has two types of classes: $i$ ) Beacon which is a simple short packet shared as an $\mathrm{OB}$, and ii) Bundle which is a collection of conventional network packets. Each Mess age structure has a size (length) defined with bytes, a creation time, and a source. If the networking objective is defined as end-to-end routing in CORE. Settings, each structure is given a destination as well. The destination Device is determined randomly in the default simulator implementation. Besides, each Message structure keeps track of several statistical information about itself such as the hop count, receiver and sender lists (the devices which sent or received the message), and dissemination count. In this way, it becomes quite straightforward to learn about the statistics of a specific Message structure at a specific time in a simulation run. Additional properties such as time-to-live, hops-to-go, time-to-send can be defined for messages in CORE. Settings.

*. Based on the author publication 


\section{B.2.2 CORE}

In this package, the core runtime functionalities of the simulator are defined in 5 classes:

\section{FileHandler}

This class is responsible for the file operations. The two main functions are as follows:

- DataParser(File, InputType) is the function where a given File is parsed based on predefined Input Type, i.e. the type which defines the data entry (a file row) format. There are two formats supported for Input Type:

- Connection, which consists of timestamp, first device ID, second device ID, and link information (link is up or link is down).

- Individual, which consists of timestamp, device ID, location information. For this format, S I M U L A I I O N . Envi ronment calculates the device-to-device connection information based on a given radio range for the utilized interface. This range can be defined manually in CORE.Preferences class.

To parse different data entry formats, new Input Type can be defined under this class.

- Dataprocessor (data) is the function where the parsed data are processed based on time variables. As further explained, this function is one of the core part of the simulator.

\section{Preferences}

The constants for the parameters defined in the classes of ABSTRACT and SIMULATION are held in this class. For instance, the value for the maximum transmission unit (MTU), which is a PHY/MAC parameter, PREFS . Network. PHYMAC.ATT_MTU, is defined under this class. The users can change these constants, or define new ones.

\section{Settings}

The functions related to simulation settings and runtime initialization are defined in this class.

\section{UI}

In the user interface (UI) class, the functions and variables related to displaying message statistics, device statistics, device movements (by playing the waypoints obtained from the mobility traces in Input Type=Individual) are defined.

\section{Utils}

This class contains several utility functions written for code efficiency related to MATLAB/Octave. 


\section{B.2.3 DATA}

This package includes the functionalities that organize the pre-collected data for the simulation. The data is related to the device properties and to the wireless operation measurements such as hotspot enable time and scan time. The data is stored in binary format, MOD E L S . Device calls the necessary functions for the data of selected device type. The device type can be defined in the preferences, for instance PREFS. Device.Model = 'Samsung S4-Mini'.

\section{B.2.4 MODELS}

In this package, device models and mobility models are defined. MODELS. Device calls the necessary functions for the data of selected device type. MODELS. Mobility generates the mobility trace file based on the selected mobility model and number of devices before the start of the simulation.

\section{B.2.5 SIMULATION}

In this package, the network environment is simulated under the following classes:

\section{Environment}

Based on the input file, the device-to-device joins and disjoins are simulated within this class. If InputType is Connection, then joins and disjoins are directly simulated over time. If Input Type is Individual, joins and disjoins are simulated based on location data and PREFS.Device.Radio. Range input.

\section{Event}

The events are simulated within this class. Each event is created with specific intervals based on the user preferences. The interval set for the event creation can be changed with the PREFS.Event. Interval tag defined in the settings. Once the event is created, it is converted to a ABSTRACT.Message, either a Beacon or Bundle based on the networking type selected, and is affiliated with a ABS TRAC T. Device.

\section{Network}

Regarding device-to-device relations, the following phases in networking are simulated within this class: $i$ ) Neighbor discovery, ii) Association, iii) Authentication, and iv) Connection. For each device in the network setup, this class is responsible to call the necessary steps in networking based on the networking type selected. The supported networking types are: $i$ ) OBN, ii) OAN, iii) Ad Hoc (classic), and iv) P2P (classic). New networking types can be implemented, or the ones available can be modified under this class. 


\section{B.3 Main Structures}

The basic structures of the simulator are explained under this section.

\section{B.3.1 DEVICE}

Each device is defined as a structure comprising fields related to Buffer, Radio, Network, and Router.

A device has a device ID, i.e. DEVICE $\{$ ID $\}$ defines a device structure. Its unique number, its initialization time in the simulator and its battery level (given randomly at the beginning) can be retrieved as follows:

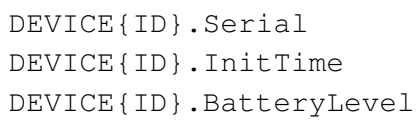

The buffer of a device structure contains the following sub-structures:

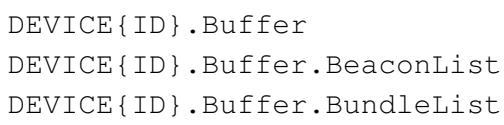

The radio of a device structure contains the following sub-structures:

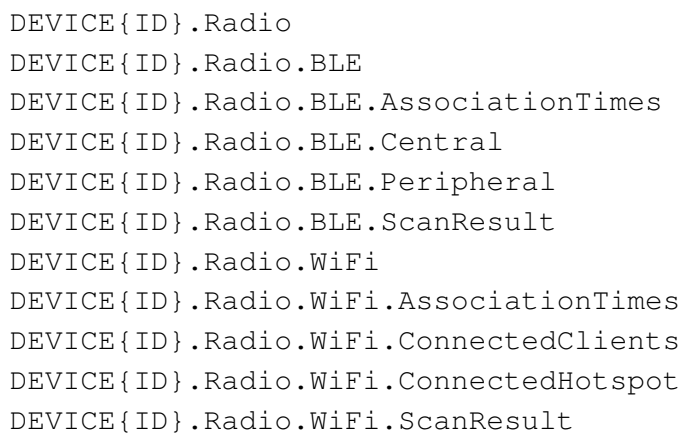

The network structure of a device structure contains the following sub-structures:

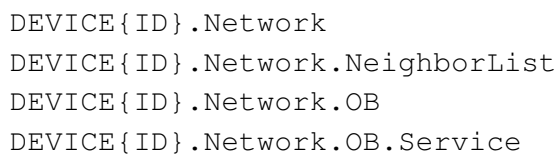




\section{B.3.4 RUNTIME}

This structure contains the runtime variables such as time, variables related to time-step functions, indices, and active devices. Some important ones are listed below:

\author{
RUNTIME \\ RUNTIME. Current \\ RUNTIME.Devices \\ RUNTIME. LastUpdate \\ RUNTIME . Next \\ RUNTIME . NextEvent \\ RUNTIME.Prev \\ RUNTIME. Start
}

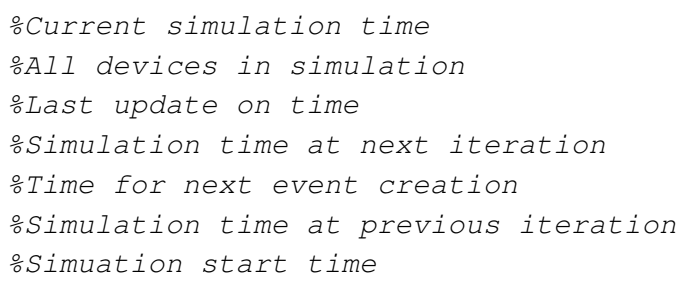

\section{B.3.5 STATS}

The runtime statistics can be obtained with this structure. It keeps track of the statistics over time, and lists the overall results in the following variables:

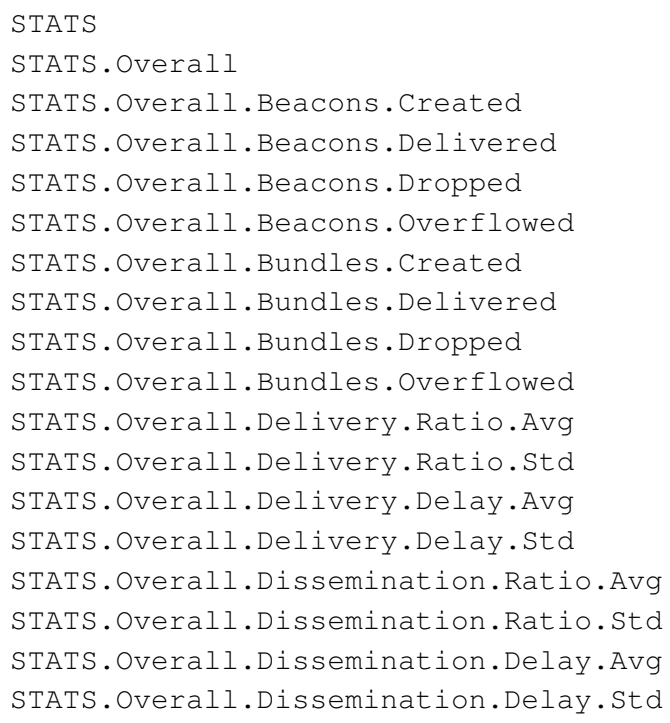

Apart from the overall result values, the same statistics are also recorded as a collection of values with respect to time, and can be retrieved from the following sub-structure:

STATS.OverTime 


\section{B.4 Operation}

To create a simulation setup, there is only a single file that a user can edit: Settings.m In this settings file, PREFS structure is used. An example setting is given in Section B.5.

To use a mobility trace file, the file must have the format of Input Type = Connection or InputType $=$ Individual. New formats must be introduced to CORE.FileHandler before using them.

The Oppliqué simulator reads the settings file, initializes the runtime variables, reads the input file (or files) by calling DataParser(), and finally creates the ABSTRACT structures just before the start of the simulation. These are handled in Run.m. In order to start the simulation, the Run command must be called in the working directory of the simulation. Then, the simulator calls Dataprocessor () function in order to iterate time. As the time iterates, it assigns and runs the jobs related to the devices.

The operation of the simulator is based on simulated events. In each given interval, an event is created in the network. Once an event is created, it is affiliated as a single unique message with a source device. In each iteration of time, the networking tasks are handled in each device.

Based on the calculated communication window between each device pair, the selected networking model is applied throughout the network setup. The execution time of the wireless operations are simulated according to the real-world experimental values that are obtained with smart mobile devices. For PHY/MAC implementation, a high level of modelling is implemented for these devices. The connection-based transmissions are based on the equation of Gupta and Kumar and the transmission speed is divided equally to all the ongoing transmissions. The connection-free transmissions, on the other hand, are based on the realworld measurements of the beacon interval and scan interval values.

The status of each device or message can be traced at any simulation time with STATS. 


\section{B.5 ExAmples}

Here, two simulation setup examples are provided. An example setting is given below:

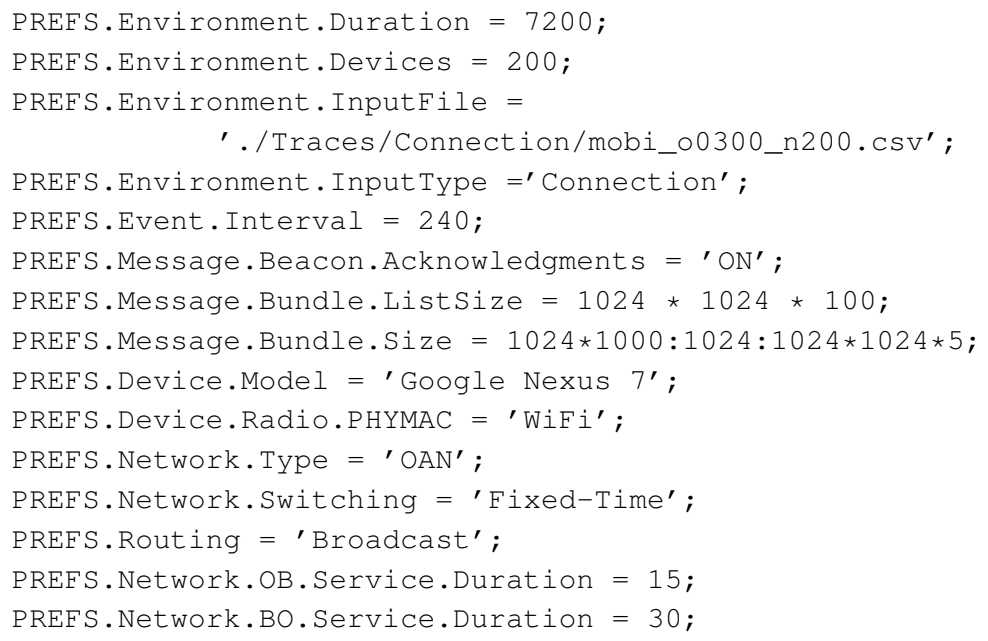

Another example regarding the setup of dual-radio utilization with time-adaptive approach given in Section 4.4.4 is given below:

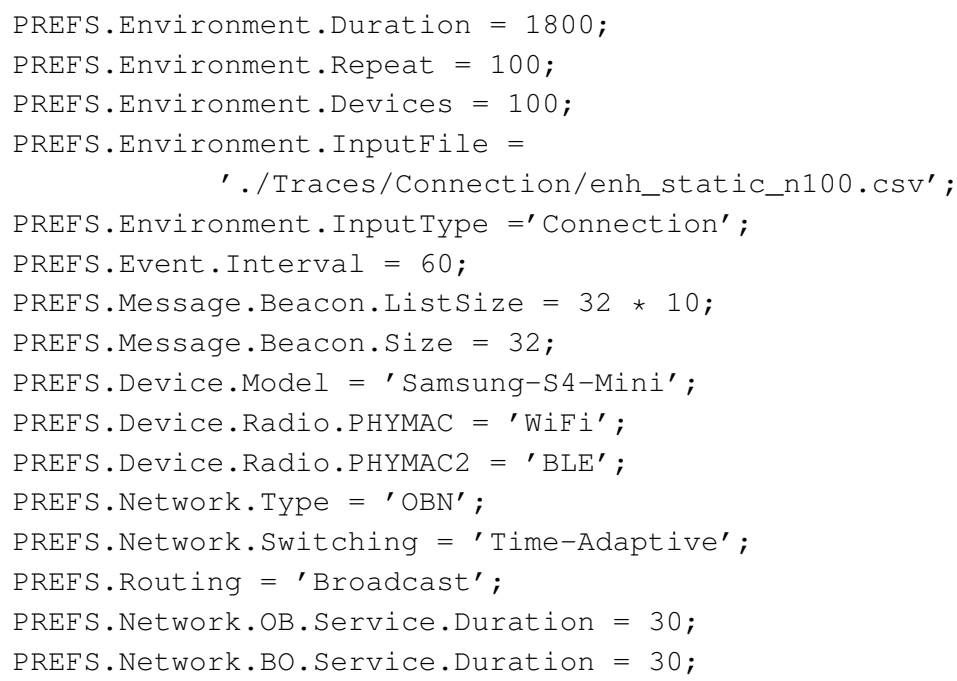



APPENDIX $C$

Cocoon

API Guide 


\section{C.1 Overview}

This guide, the Cocoon API guide, describes the status of the Cocoon implementation on the Android platform at the time of this writing. Furthermore, it briefly describes how to develop Cocoon applications.

This guide assists interested users (e.g. students, programmers) or communities (e.g. software developers, mobile application builders, teams, companies focusing on niche business models by implementing opportunistic social networking applications) to develop or use their own Cocoon-based implementations based on their specified application areas. The guide includes the very brief descriptions of the packages, functions, and variables of the Cocoon API. The Cocoon API contains the implementation of generic ad hoc networking protocols as well as includes the implementation of Opportunistic Beacon Networking (OBN) and Opportunistic Association Networking (OAN) presented in this thesis. Therefore, this guide assumes familiarity with the network programming and with the Cocoon architecture. All protocols are open for public use. Apart from networking, it also includes a set of sensing tools (yet limited for now) to enrich the context of the applications.

With the utilization of the Cocoon library, developing ad hoc opportunistic systems becomes easier without requiring modifications on the default Android platform.

The definitions and examples given in this appendix are based on the implementation at the time of this writing, therefore online documents in the future may supersede this guide.

The most up-to-date technical documentation can be reached on the author's Github page: https://github.com/okanturkes/cocoonapi

Cocoon is developed in Java programming language for Android platforms. It is implemented as a service that can be attached to an application. By using this service, an application inherits all the functionalities of the Cocoon service. The service includes generic service definitions (variables and metrics) and routines that are together presented as an API. The Cocoon API can be separately utilized in order to develop applications independent from the Cocoon implementation. The Cocoon API is an instrumental inter-layer in between the application and network layers. It provides an ease-of-applicability to its users in developing ad hoc networking applications.

The rest of the chapter is as follows: C.2 describes the packages. C.3 presents how an example application is built by using the Cocoon API. Finally, C.4 gives an example application using the Cocoon implementation. 


\section{C.2 Packages}

In this section, the details of each package are given. The Cocoon API has 10 main packages:

- Constants

- Defaults

- Types

- INBOX

- BeaconList

- BundleList

- Interface

- Dialog

- ListAdapter

- Notification

- Vibration

- LOG

- Offlinelogger

- Onlinelogger

- Message

- Beacon

- Bundle

- Format

* Field

* ReservedField

- MONITOR

- Beaconobserver

- OpportunisticBeacon

- Switcher
- NETWORK

- BleManager

* Central

* Peripheral

* Ble

- WifiManager

* Client

* Hotspot

* Wifi

- Router

- BleRouter

- WifiRouter

- Service

- Cocoon

- Configuration

- MainService

- Timer

- Utils

- Baseutils

- Dateutils

- DeviceUtils

- FileUtils

- Serverutils

- Stringutils

- Timeutils

\section{C.2.1 Constants}

The package Constants includes service-specific constant values, types, and strings. In this package, two classes are defined: Defaults and Types. Defaults contains the default values related to the service runtime. Types contains the types related to service, message formats, and wireless states.

\section{C.2.2 InBOX}

The Inbox package has two types of classes: $i)$ BeaconList which is a buffer for simple short packets shared in the network, and ii) BundleList which is a buffer for the collection of conventional network packets. Each class contains the buffer management functions.

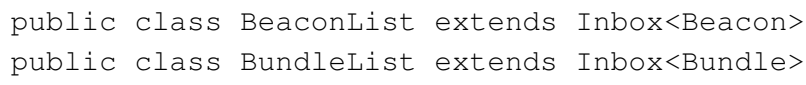




\section{C.2.3 INTERFACE}

The package Interface includes the utility classes and functions related to the user interface. This package is not used by the Cocoon service, however can be used in Cocoon-based application development.

\section{C.2.4 LOGGER}

The package Logger can be used in logging activities in other functions. The Cocoon service uses Offlinelogger in order to document the runtime. If required, these logs can be stored in the device. Onlinelogger, on the other hand, can be used to upload these logs to a web server. The settings of the web server (e.g. IP, port) can be defined in this class. In the Cocoon implementation, or in its proprietary applications (also in the applications presented in this thesis), onlinelogger is never used under any circumstances in an attempt to violate the right of privacy.

\section{C.2.5 MESSAGE}

The package Message contains two classes for the definitions of Beacon and Bundle implementations and a sub-package for the Format classes.

The Beacon class defines a beacon with its format generated in accordance with the Field and ReservedField classes. The fields of a beacon are generated with respect to the identifier encoding presented in Section 3.3.2.

The Bundle class defines a bundle, which is a collection of packets used in connectionbased routing. There is no type defined for the bundles.

\section{C.2.6 MONITOR}

In the package Monitor, the OpportunisticBeacon, Beaconobserver, and Switcher classes inherit Java's Broadcast Receiver class in order to monitor wireless states such as Wi-Fi on, Wi-Fi off, BLE Periphral on, BLE Central on. Each of the classes use a shared timer in order to keep track of the wireless statuses. Based on the given settings, the timer adjusts the OB-BO states. These classes are initiated as basic objects in order to establish the network connectivity in the Cocoon service.

\section{C.2.7 NETWORK}

The package Network includes Cocoon-specific functions rewritten on top of the Wi-Fi and BLE managers defined in the default Android API.

\section{BleManager}

The sub-package BleManager includes classes related to the BLE interface. 
Ble class includes the BLE manager. It helps to switch to different BLE states, or to check the current BLE states.

Central can be utilized when the current wireless service type of the device equals to beacon observer. It includes functions to search BLE peripheral devices, to store specific BLE beacons, and to list the specifications of the connected devices.

Peripheral can be used when the current wireless service type of the device equals to opportunistic beacon. The class includes functions to list and manage connected BLE central devices.

\section{WifiManager}

The sub-package WifiManager includes classes related to the Wi-Fi interface.

Wifi class includes the Wi-Fi manager. It helps to switch to different Wi-Fi states, or to check the current Wi-Fi states.

Client can be utilized when the current wireless service type of the device equals to beacon observer. It includes functions to search Wi-Fi networks, to store specific Wi-Fi beacons, and to list the specifications of the connected hotspot.

Hot spot can be used when the current wireless service type of the device equals to opportunistic beacon. The class includes functions to list and manage connected Wi-Fi clients.

\section{C.2.8 ROUTER}

The package Router includes two classes: In BleRouter, the connection-based BLE functions are defined. In WifiRouter, the connection-based Wi-Fi functions are defined.

\section{C.2.9 Service}

The Service package is the core part of the Cocoon implementation. It includes the following:

- Cocoon, an extended Application class.

- Configuration, the runtime configuration class.

- MainService, the class in which the connectivity management, network management and QoS management are handled.

- Timer, the class which deals with all time-based operations during runtime.

\section{C.2.10 UTILS}

This package contains several utility classes written for code efficiency related to the Cocoon implementation. 


\section{C.3 Application Building}

This section discusses on two different ways of application building with the Cocoon library.

One way of application building could be utilizing the built-in Cocoon and Ma inService instances.

Using the built-in Cocoon class, the following explains how to inherit Cocoon's Appl i cat ion class instance as the parent application class. Under the package Service, the Cocoon class includes the already-initialized parameters related with the other packages and corresponding managers, monitors, and operations once it is extended by an application class:

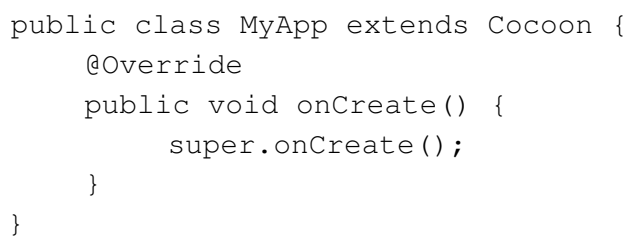

In Android's manifest file, the application name must be defined as the base package name. Thus, all initialized parameters of Cocoon can be used.

<application android:name="MyApp" ...>

On the other hand, MainService under the package Service initiates two background operations by default: Timer and Switcher. By initiating MainService, as given in the example below, you application is ready to run the Cocoon routing:

MyApp.startService (MyActivity.this);

Nevertheless, MyApp must include to the Cocoon instance at least one Inbox variable and at least one BeaconFormat variable in order to make advertisement and scanning operations work properly.

The second way could be building an application from scratch by utilizing the packages independently but interdependently. Apart from the package Service, other packages can be used independently as well to create an application from scratch. 


\section{C.4 EXAMple}

In the following, the implementation of a Hello World application is provided. The application is an opportunistic Twitter application using Opportunistic Beacon Networking. That is, the messages (tweets) are shared via the wireless identifiers. As the wireless interface, $\mathrm{Wi}-\mathrm{Fi}$ is used. Thus, the messages are encoded in the SSID fields. The beacon format is based on the identifier encoding presented in Section 3.3.2. The first 12 bytes are reserved for the Cocoon service. The payload, which is 20 bytes, is divided into 3 fields as follows:

- Username (5 bytes), to identify the owner of the message.

- Tweet (14 bytes), for the message (instead of Twitter's 140 characters).

- Mood (1 byte), to additionally include the user's mood. In order to encode mood in 1 byte, a mood decoder must be included within the application. However, this is not included in this example.

For the message fields, the new classes are defined. The classes inherits the Field class. As shown in the following files, encode ( ) and decode ( ) functions in these classes are left empty for further implementation. This example only shows how the beacon format is generated for the given application.

The application uses the default configuration parameters of the inherited service. However, some configurations can be changed in the application, such as scan interval as done in the main activity class.

The application files are provided in the following pages:

\section{HelloWorld.java}

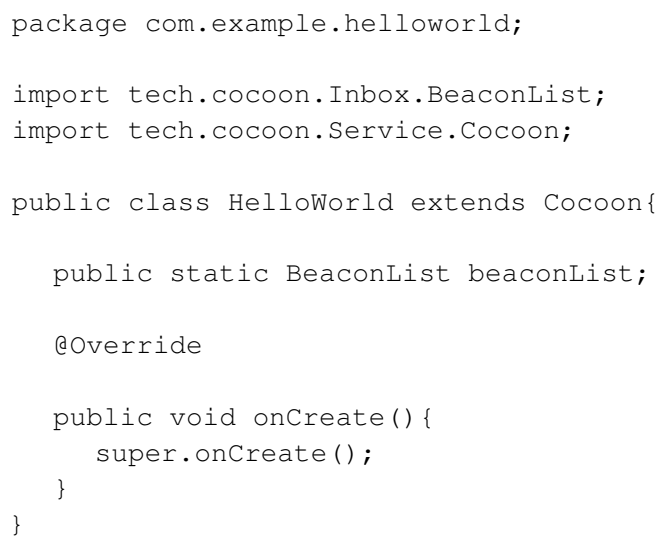




\section{MainActivity.java}

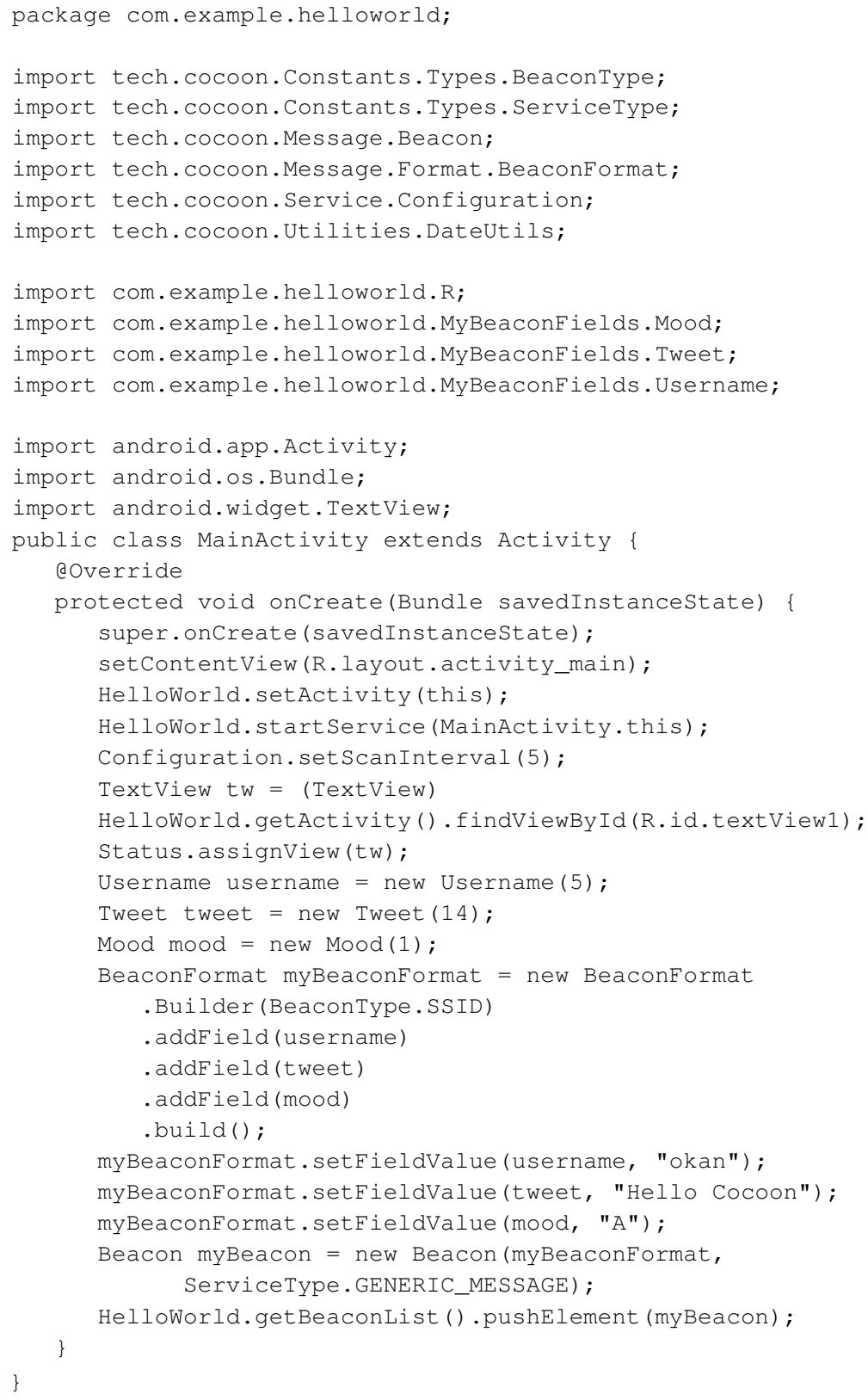




\section{Username.java}

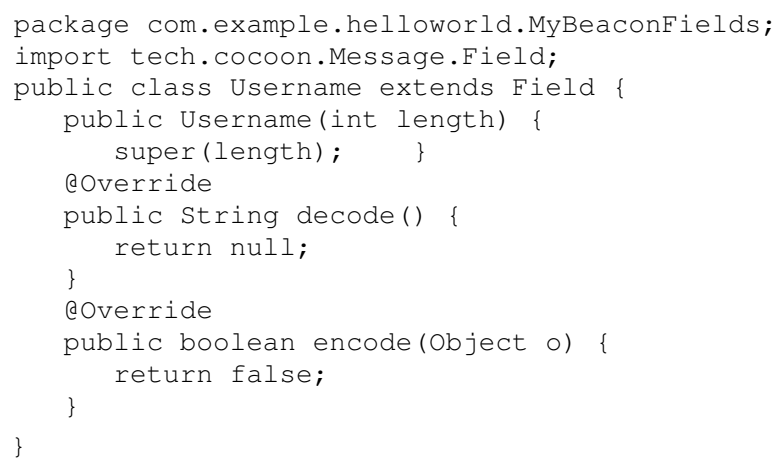

\section{Tweet.java}

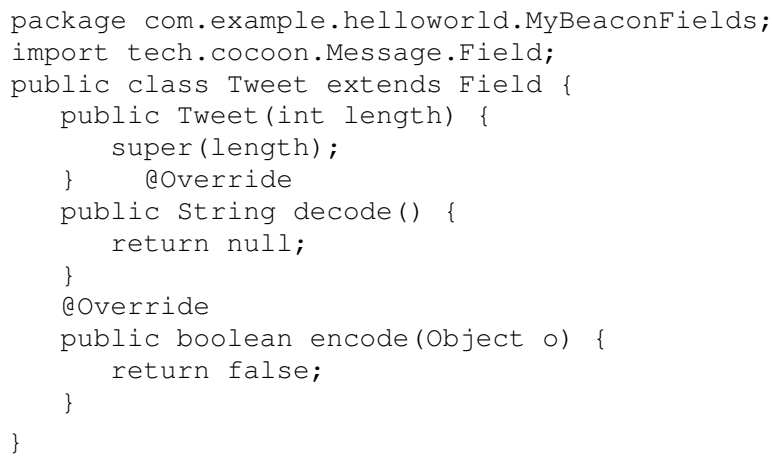

\section{Mood.java}

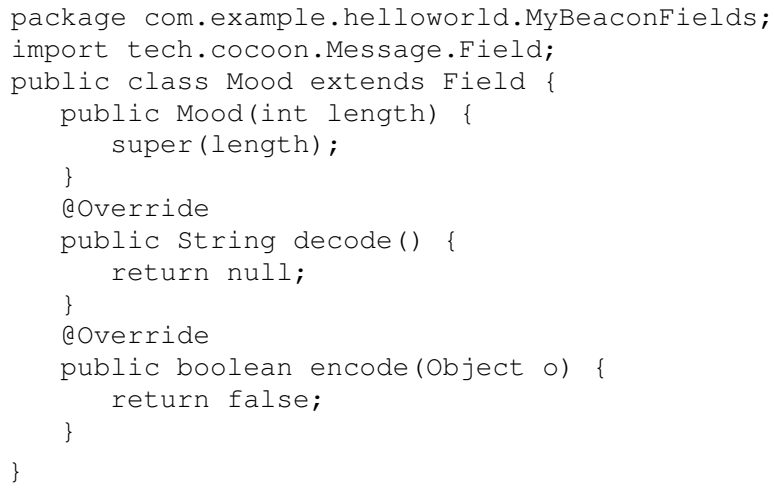




\section{INDEX}

ad hoc networks, 1, 3, 26, 35, 68, 89, 113, 124, 128

American Standard Code for Information Interchange (ASCII), 27, 28, 57, 58, 116

see also SSID

Analytic Network Process (ANP), 63

Android, 15, 31-34, 37, 40, 59, 95

Apple, 128

for iOS, see iOS

iBeacon, 37

Application Programming Interface (API), 33, 40, 55

battery percentage usage (BPU), 60 see also energy efficiency

Beacon Observer (BO), 49, 53, 56, 59, 62, 71, 95, 96, 113,125

beacons, 35, 36, 49, 57, 59, 123, 130

for Cocoon beacon, see Cocoon beacon format

AltBeacon, 37

EddyStone, 37

iBeacon, 37

BlackBerry BBOS, 31

Bluetooth, 15, 45, 125, 130

Classic, 8, 27, 29, 33, 36, 57, 127

master/slave, 27,28

network name, 36, 57

piconet, 16,27

scatternet, 27

Clock Synchronization Protocol, 61

Low Energy, Smart (BLE), 15, 27, 34, 36, 57, $59,93,130$

Central mode, 27, 59, 106

GATT, 29

Peripheral mode, 27, 59, 106

Low energy, Smart (BLE), 37

Cocoon, 1, 18, 38, 40, 47, 57, 93, 121, 123, 125, 130
API, 20, 51, 73, 127, 157

see also Appendix C

beacon format, 97,98

see also Opportunistic Beacon

contact diversity (CD), 55, 58, 63, 97

contact stability (CS), 55, 58, 63, 97

context awareness, 18, 24, 25, 42, 45, 54, 129

data dissemination, $18,19,50,53,57,63,69,78-80$, $85,87,112,113,129$

see also routing

data diversity (DD), 55, 58, 63, 97

data stability (DS), 55, 58, 63, 97

Delay Tolerant Network (DTN), 4, 126

energy efficiency, 4, 14, 59-61, 127

forwarding, 4, 18, 23, 25, 41, 54, 57

see also routing protocols

Google, 128

for Android, see Android

EddyStone, 37

Haggle, 33, 34, 40

IEEE 802.11, 17, 26, 59, 92

for SSID, see SSID

for $\mathrm{Wi}-\mathrm{Fi}$, see Wi-Fi

802.11p, 112

Basic Service Set (BSS), 26, 28, 112

Carrier Sense Multiple Access, 61

Collision Avoidance (CA), 61

IEEE 1609, 112

WAVE BSS, 112

IEEE 802.15.1, 17, 26

for Bluetooth, see Bluetooth

infrastructure, 9, 38, 129

access point (AP), 2, 26, 36, 112, 113, 121, 129

cellular base station, 2, 9, 33, 94, 121

road-side unit (RSU), 9, 112, 121, 129 
iOS, 31, 32, 34, 37, 40, 59, 95

mesh networks, 33, 35, 36, 112

Microsoft, 128

for Windows Phone, see Windows Phone

Mobile Ad Hoc Network (MANET), 3, 6, 35

mobility, 3, 4, 6, 7, 24, 29, 43, 45, 61

neighbor discovery, 17, 28, 29, 38, 50, 56, 61, 86, 130

network density, 29, 38, 61, 127, 129

Open Garden, 33, 34, 40

FireChat, 36

Oppliqué, 20, 73, 99, 118, 127, 145

see also Appendix B

Opportunistic Association Networking (OAN), 50, 61

Opportunistic Beacon (OB), 17, 49, 50, 53, 56, 59, $62,71,95,96,113,125$

Opportunistic Beacon Networking (OBN), 36, 38, $50,53,60,61,95,112,124,125,127$

Opportunistic Network (OppNet), 7, 8, 49, 63 applications, 9, 40, 93, 112, 120, 126, 130 characteristics, 13, 24, 27, 28, 35

requirements, $14,24,38,48,50,63,125$

see also systems

opportunistic sensing, 44, 50, 125

packet switching, 49, 62, 116

peer-to-peer (P2P), 1, 8, 13, 34, 36, 112

Physical \& Media Access Control (PHY/MAC), 17, $38,52,61,99,112$

piconet, 36

Pocket-Switched Networks, 33

quality-of-service (QoS), 18, 52, 57, 58, 63, 64, 127, 129

replication, $6,25,41$

routing, 3, 4, 14, 42, 45, 57, 58, 63, 95, 125, 129 delivery delay, 4, 5, 89, 108, 114, 121, 128, 129

delivery probability, 5, 38, 41, 101, 102, 105, $109,126,129$

hop count, $6,54,58$

protocols, 23, 24, 82, 129

scalability, 14, 15, 126

time-to-live (TTL), 6, 54, 58

time-to-sent (TTS), 6

see also data dissemination

scalability, 4, 49, 56, 63, 69, 70, 79, 80, 114, 121, 125, 129,130
Serval, 33, 40

The Serval Mesh, 33

Service Set Identifier (SSID), 28, 35-37, 50, 57, 58, $62,73,95,109,112,113$

smart mobile devices, 2, 8, 15, 24, 26, 33, 38

Google Nexus 7, 59, 60

Motorola Moto G, 59, 60, 99

Samsung S2, 59

Samsung S4 Mini, 59, 61, 99, 118

Stability Index, 65

store-carry-forward, 4, 5, 7, 53, 70 see also forwarding

systems, 113

for Cocoon, see Cocoon

for Haggle, see Haggle

for Open Garden, see Open Garden

for Serval, see Serval

see also Opportunistic Network (OppNet) applications

throughput, $18,38,50,57,71,89,108,113,114,127$

Unicode Transformation Format 8-bit (UTF-8), 27, 28,57

Universally-Unique Identifier (UUID), 28, 50, 57, $58,73,109$

Update \& Switch (U\&S), 53, 71, 84, 95

Wi-Fi, 8, 15, 45, 59, 99, 113, 125, 130

Ad Hoc mode, 15, 26, 33, 38, 40, 61, 127

Aware, 130

Direct, 15, 26, 28, 34, 36, 40

Halow, 130

Hotspot mode, 15, 26, 35, 38, 59, 94, 106, 113

Infrastructure mode, 28, 35, 59, 60, 94, 99, $106,112,113$

WiFi-Opp, 35, 38, 40, 61, 113

Windows Phone, 31-33, 40, 95

Wireless Local Area Network (WLAN), 1, 8, 26, 35, 57

channels, 35, 59

wireless operations, 59, 73, 95, 125

beacon interval, 59, 71, 114

beaconing, 38, 49, 50, 53, 54, 56, 59, 61, 70, $71,73,83,84,94,95,99,121$

scan interval, 59, 71, 114

scanning, 26, 56, 59, 70, 71, 73, 83, 84, 94, 95, 121,127

active/passive, 28,59

switching, 54, 57, 60, 72, 73, 83, 94, 95, 113

Wireless Personal Area Network (WPAN), 1, 8, 26, 35,57

Wireless Sensor Network (WSN), 7 



\section{About the Author}

OKAn Türkeş was born in Samsun, Turkey, in 1986. He graduated from Samsun Anatolian High School with high honors in 2004. In 2009, he received his Bachelor of Science (BSc) degree in Computer Engineering from Yeditepe University, İstanbul, Turkey. For his graduation project, he worked on the topic of Human-Computer Interaction within the VISPLAT project ${ }^{1}$. In 2010, he obtained a minor Bachelor of Arts (BA) degree in Business Administration at the same university. His Master of Science (MSc) degree followed in 2012, also in Computer Engineering for the research he performed at the Wireless Networks Laboratory of Yeditepe University. The focus of this research, supported by Turkcell, was on multimedia encoding and transmission in Wireless Sensor Networks. Same year, he started as a PHD candidate at the Centre of Telematics and Information Technology, University of Twente, Enschede, the Netherlands, as a member of the Pervasive Systems group. In parallel with his PHD research, he was primarily involved in SENSAFETY project within the context of the Dutch National Program, COMMIT ${ }^{2}$, as well as partially worked in ILAND project within the ARTEMIS research programme ${ }^{3}$ and RECONSURVE project organized by the ITEA $3 /$ EUREKA programme $^{4}$. In connection with these projects, his research interests include wireless ad hoc networks, opportunistic networking \& sensing. His professional interests center on the research and development of mobile communication systems, in particular systems for smart-networked objects and Internet of Things.

\footnotetext{
1. VISPLAT: http://cse.yeditepe.edu.tr/vr_web/visplat.php

2. SenSafety: http://www.sensafety.nl, COMMIT: http://www.commit-nl.nl

3. iLAND: http://artemis-ia.eu/project/10-iland.html, ARTEMIS: http:/ /artemis-ia.eu

4. RECONSURVE: http://www.reconsurve.eu, ITEA: http://itea3.org
} 
List of publications in which he participated are as follows in reverse chronological order:

- Türkeş, O. and Scholten, H. and Havinga, P. J. M. Cocoon: A Lightweight Opportunistic Networking Middleware for Community-oriented Smart Mobile Applications, Computer Networks, Special Issue on Cyber-Physical Systems for Mobile Opportunistic Networking, under final revision, to be published in September 2016.

- Türkeş, O. and Scholten, H. and Havinga, P. J. M. Friend-to-Friend Short Message Service with Opportunistic Wi-Fi Beacons, in Proceedings of the 7th IEEE International Pervasive Computing and Communications (PerCom) Workshop on Pervasive Collaboration and Social Networking (PerCol 2016), Sydney, Australia, March 2016, ISBN: 978-1-5090-1941-0.

- Türkeş, O. and Scholten, H. and Havinga, P. J. M. Opportunistic Beacon Networks: Information Dissemination via Wireless Network Identifiers, in Proceedings of the 5th IEEE International Pervasive Computing and Communications (PerCom) Workshop on the Impact of Human Mobility in Pervasive Systems and Applications (PerMoby 2016), Sydney, Australia, March 2016, ISBN: 978-1-5090-1941-0.

- Türkeş, O. and Baydere, Ş. Priority-Based Voice Segmentation and Transmission in Quality-Driven Wireless Audio Sensor Networks, Ad Hoc Sensor Wireless Networks, Volume: 29(1-4), December 2015, 311-331, ISSN: 1551-9899.

- Türkeş, O. and Scholten, H. and Havinga, P. J. M. BLESSED with Opportunistic Beacons: A Lightweight Data Dissemination Model for Smart Mobile Ad-Hoc Networks, in Proceedings of the 10th ACM Mobile Computing and Networking (MobiCom) Workshop on Challenged Networks (CHANTS 2015), Paris, France, September 2015, 25-30, ISBN: 978-1-4503-3543-0.

- Türkeş, O. and Seraj, F. and Scholten, H. and Meratnia, N. and Havinga, P. J. M. An Ad-Hoc Opportunistic Dissemination Protocol for Smartphone-Based Participatory Traffic Monitoring, in Proceedings of the 82nd IEEE International Vehicular Technology Conference (VTC 2015 Fall), Boston, MA, USA, September 2015, 1-5, ISBN: 978-1-4799-8091-8.

- Seraj, F. and Zhang, K. and Türkeş, O. and Meratnia, N. and Havinga, P. J. M. A Smartphone Based Method to Enhance Road Pavement Anomaly Detection by Analyzing the Driver Behavior, in Proceedings of the 4th ACM International Pervasive and Ubiquitous Computing Workshop on Pervasive Urban Applications (PURBA 2015), Osaka, Japan, September 2015, 1169-1177, ISBN: 978-1-4503-3575-1. 
- A. K. Demir, A. K. and Türkeş, O. and and Baydere, Ş. Differentiating Emergency Voice Traffic in Indoor Wireless Activity Monitoring Network, in proceedings of the 10th IEEE International Conference on Wireless and Mobile Computing, Networking and Communications (WiMob 2014), Larnaca, Cyprus, October 2014, 598-603, ISBN: 978-1-4799-5041-6.

- Türkeş, O. and Scholten, H. and Havinga, P. J. M. RoRo-LT: Social Routing with Next-Place Prediction from Self-Assessment of Spatiotemporal Routines, in Proceedings of the 10th IEEE International Conference on Ubiquitous Intelligence and Computing (UIC 2013), Vietri Sul Mare, Italy, December 2013, 201-208, ISBN: 978-1-4799-2481-3.

- Türkeş, O. and Scholten, H. and Havinga, P. J. M. Introspection-based periodicity awareness model for intermittently connected mobile networks, in Proceedings of the 4th International Conference on Mobile, Ubiquitous, and Intelligent Computing (MUSIC 2013), Gwangju, South Korea, September 2013, 397-403, ISBN: 978-3642-40674-4.

- Türkeş, O. Opportunistic Data Dissemination in Mobile Phone Sensor Networks, in Adjunct Proceedings of the ACM Pervasive and Ubiquitous Computing (UbiComp) Doctoral School (UbiComp-DS 2013), Zurich, Switzerland, September 2013, Extended Abstract (4 pages), ISBN: 978-1-4503-2139-6.

- Türkeş, O. and and Baydere, Ş. Towards inherent error-resilient voice encoding schemes in audio sensor networks, in Proceedings of the 7th ACM International Mobile Computing and Networking (MobiCom) workshop on Wireless network testbeds, experimental evaluation and characterization (WiNTECH 2012). Istanbul, Turkey, August 2012, 35-42, ISBN: 978-1-4503-1527-2.

- Türkeş, O. and and Baydere, Ş. Voice Quality Improvement with Error Concealment in Audio Sensor Networks, in Proceedings of the 10th International Conference on Wired/Wireless Internet Communication (WWIC 2012), Santorini, Greece, June 2012. 307-314, ISBN: 978-3-642-30630-3.

- Türkeş, O. and and Baydere, Ş. Voice quality analysis in wireless multimedia sensor networks: An experimental study, in Proceedings of the 7th IEEE International Conference on Intelligent Sensors, Sensor Networks and Information Processing (ISSNIP 2011), Adelaide, Australia, December 2011, 317-322, ISBN: 978-1-45770675-2. 

